\begin{abstract}
ANL-94/31
ARGONNE NATIONAL LABORATORY

9700 South Cass Avenue

Argonne, Illinois 60439-4801
\end{abstract}

\title{
PHYSICS DIVISION ANNUAL REPORT
}

April 1, 1993 -- March 31, 1994

Walter F. Henning

Director

August 1994

Preceding Annual Reports

ANL-91/12 1990-1991

ANL-92/16 1991-1992

ANL-93/12 1992-1993 


\section{FOREWORD}

The past year has seen major progress in the various areas of research within the Argonne Physics Division. This report gives a glimpse of the many activities and programs under way. For more detailed information you are encouraged to request preprints and/or reprints. The easiest way to contact us would be through e-mail, thayer@anlphy.phy.anl.gov.

Perhaps foremost among the accomplishments during the past year was the full-scale, 7-day operation of ATLAS with the new positive-ion injector. Intense ion beams are now available for all atoms up to uranium. Heavy beams took more than $50 \%$ of the beam time, with uranium beams alone comprising about $20 \%$. The 7-day operating schedule was made possible with incremental funds, initially from the Laboratory and in FY1994 from the DOE program office.

In addition, major progress in accelerator performance was achieved through a number of improvements: the charge-state selector to isolate a particular ion after stripping behind the positive-ion injector; various improvements in the ECR source including the low-temperature oven for solid-material beams and laserablation techniques; significant improvement in the performance and reliability of the fast tuner system; and startup work on the second advanced ECR ion source with stronger magnetic field and higher operating frequency.

Studies in rf superconductivity are continuing. A prototype cylindrically symmetric quarter-wave line structure was constructed as part of the joint project with the Nuclear Science Centre, Delhi, India. The construction of a niobium superconducting radiofrequency quadrupole structure is nearing completion, in collaboration with AccSys Technology Inc. of Pleasanton, California and funded as a Small Business Innovation Research grant. Production of superconducting cavities for the University of Sao Paulo, Brazil, is also nearly complete.

A broad program continues in low-energy heavy-ion nuclear physics research. A major part of the research effort is devoted to gamma-ray experiments and studies in superdeformation. This involves the use of the Argonne-Notre Dame BGO setup as well as experiments on the external detectors GAMMASPHERE and EUROGAM. A new approach to study the strikingly sudden decay out of the superdeformed band was devised by the Argonne group. By observing the complete spectrum of gamma rays characterizing this decay a quasicontinuous distribution was observed with superimposed broad structures as well as sharp peaks. This shows that the decay originates from the coupling between the superdeformed states with the sea of normal states in which they are embedded, providing a case study for the coupling of a cold ordered system into a hot chaotic one.

A second major research program is the study of exotic nuclei far from the valley of beta stability using the Argonne Fragment Mass Analyzer (FMA). The FMA provides excellent background suppression and high detection efficiency. One recent highlight of this research is the discovery of the heaviest proton emitter known, ${ }^{167} \mathrm{Ir}$.

The ATLAS Positron Experiment (APEX), a second-generation experiment to study the effects of strong electromagnetic fields in close collisions of high-Z atoms, was completed and progressed from the testing phase to full operations over the past year. Initial experience showed that, in essentially all respects, the apparatus performs according to the design specifications. Beams of $238 \mathrm{U}$ of intensity up to 5 pnA from ATLAS were used to bombard ${ }^{181}$ Ta tirgets and more than 400,000 positron-electron coincidences were recorded.

The Argonne medium-energy nuclear physics program has a major presence in many important research programs at outside accelerators. In experiment E665 at Fermilab, the deep inelastic scattering of 490$\mathrm{GeV}$ muons at Fermi National Accelerator Laboratory measures the changes of the quark distributions in the nucleus compared to the nucleon, and the interactions of high-energy quarks and hadrons with nuclear matter. New results on the distributions of high-energy-transfer events with distinct jets of hadrons suggest, for the first time, the possibility of significant modifications of the gluon distributions in the nucleus. In exclusive $\rho$-meson production, a clear signal for color transparency is observed. 
Considerable technical resources of the medium-energy program were devoted to developing a new technology for producing polarized hydrogen and deuterium targets, employed in electron storage rings to study spin-dependent phenomena at high momentum transfer. A collaboration between Argonne and Novosibirsk is engaged in a program of tensor polarization measurements. The program at Novosibirsk provides a proof-of-principle for the feasibility of the HERMES experiment, a broadly-based North American-European collaboration involving Argonne, to study the spin structure of the nucleon using internal polarized targets in the HERA electron storage ring at DESY.

The Argonne medium-energy physics program has a major presence in the research program at CEBAF. Staff memhers are ?ctively involved in construction of the experimental facilities, and are spokesmen for five experiments that were approved for running when beam becomes available. Members of the grcup assumed responsibility for the construction of a broad-purpose short-orbit spectrometer (SOS) to be made generally available to the users at CEBAF. Commissioning of the SOS is planned for the fall of 1994.

The nuclear theory program in the Physics Division focused on recent theoretical questions raisf by the new facilities employing electrons and heavy ions. The meson-exchange Hamiltonian model which has been developed over the years is very successful in resolving the problem of threshold $\mathrm{pp} \rightarrow \mathrm{pp} \pi^{\mathrm{0}}$. To address physics at CEBAF, effort is continuing to extend the model to include higher mass nucleon resonances $\left(\mathrm{N}^{*}\right)$ and the production of heavy mesons. The effort to develop the Dyson-Schwinger equation approach to quantum (hromodynamics, which is an alternative to lattice gauge theory in describing nonperturbative dynamics, is proceeding apace. The model was used successfully to describe the electromagnetic pion form factor at space-like momentum-transfer and $\pi$ - $\pi$ scattering up to about 600 $\mathrm{MeV}$.

The theoretical program on nuclear forces and nuclear systems focused on the construction of realistic twoand three-nucleon potentials and the development of many-body techniques for computing nuclear properties with these interactions. A new nucleon-nucleon potential, Argonne $\mathrm{v}_{18}$, was developed over the last two years which includes charge-dependent and charge-asymmetric terms. A significant development in the last year was the conversion of the few-body and light-nuclei computer programs to run on Argonne's new 128-processor IBM SP1 parallel supercomputer at speeds of $\sim 6$ billion floating point operations per second. This made extensive calculations of ${ }^{40} \mathrm{Ca}$ possible for the first time, as well as much more thorough studies of ${ }^{6} \mathrm{Li}$ and ${ }^{16} \mathrm{O}$. Work also continues on studies of superdeformation and halo nuclei like ${ }^{11} \mathrm{Li}$.

The Atomic Physics research focused on two principal areas: (a) Accelerator-based atomic physics, (b) Synchrotron radiation-based atomic physics. The ATLAS-based program included studies of atomic structure through direct wavelength measurements and lifetime measurements of helium-like heavy ions. The experiments are testing new calculations of relativistic and quantum-electrodynamic many-body interactions. In addition, ultrahigh precision measurements of the lifetimes of excited states in alkalis were performed by two laser-based methods: fast-beam laser and time-correlated single-photon counting. The results establish the many-body perturbation theory calculations of the dipole matrix element in Cs at the $0.5 \%$ level. Highly ionized xenon beams from ATLAS were used to study the ionization and fragmentation of the fullerene, $\mathrm{C}_{60}$. The Coulomb-explosion program using cold molecular beams from the Dynamitron, concentrated on the study of large-amplitude nuclear vibrational motions in several small polyatomic molecules.

Synchrotron-based atomic physics measurements were carried out at the Brookhaven National Synchrotron Light Source and focused efforts are under way to establish a novel atomic and molecular program at the 7-GeV Advanced Photon Source (APS) at Argonne, currently under construction.

As in all the past years, the scientific vitality and the intellectual climate in the Division was greatly enhanced and enriched by visitors, both short term and long term, and a large number of enthusiastic students. The latter range from students just graduated from high school (Pre-College Program) to undergraduates and resident graduate students in all of the research areas.

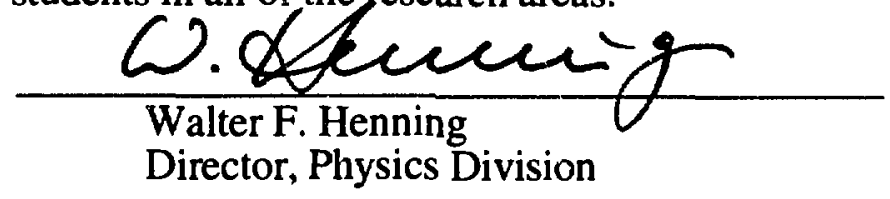




\section{TABLE OF CONTENTS}

I. HEAVY ION NUCTEAR PIYSICS RESE

I. HEAVY-ION NUCLEAR PHYSICS RESEARCH..................1

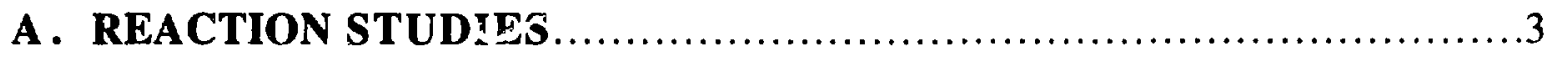

a. Quasi-Elastic and Deep-Inelastic Keactions Close to the

Coulomb Barrier........................................................ 3

a.1. Observation of the One-To Six-Neutron Transfer Reactions............4

a.2. The Influence of Transfer Reactions on the Sub-Barrier

Fusion Enhancement in the Systems ${ }^{58,64} \mathrm{Ni}+{ }^{92,100} \mathrm{Mo} \ldots \ldots \ldots \ldots . .5$

a.3. Energy Dependence of Neutron-Transfer Reactions in the Systems $98,100 \mathrm{Mo}+{ }^{58} \mathrm{Ni}$ Below the Coulomb Barrier ...............5

a.4. Neutron Transfer at Large Distances in Spherical and

Tleformed Systems ...........................................

a.5. Transfer Reactions in the Systems ${ }^{28} \mathrm{Si},{ }^{37} \mathrm{Cl}+{ }^{208} \mathrm{~Pb}$ at

Energies Above the Barrier.....................................

a.6. Deep Inelastic Scattering Near the Coulomb Barrier ..................7

a.7. Charge-State Dependence of Internal Conversion in ${ }^{83} \mathrm{Kr}$..............7

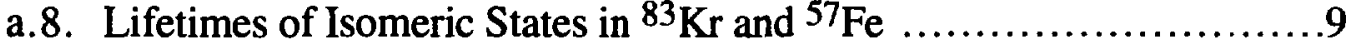

b. Search for Exotic Cluster States in Light Nuclei................... 10

b. 1. Study of the ${ }^{12} \mathrm{C}+{ }^{12} \mathrm{C} \rightarrow{ }^{8} \mathrm{Be}_{\mathrm{g} . \mathrm{s} .}+{ }^{16} \mathrm{O}(4 \alpha)$ Reaction ............. 10

b. 2. $20 \mathrm{Ne}+20 \mathrm{Ne}$ Elastic and Inelastic Scattering .................... 12

b.3. Search for Many-Body Decay of Resonances in the

${ }^{24} \mathrm{Mg}+{ }^{24} \mathrm{Mg}$ System ...................................... 14

c. Fusion and Fission Reactions................................... 14

c. 1. Measurement of Fusion Cross Sections for $58,64 \mathrm{Ni}+92,100 \mathrm{Mo}$ Using the Gas-Filled Magnet Technique ....................... 15

c.2. Measurements of Fusion Cross Sections in the Systems

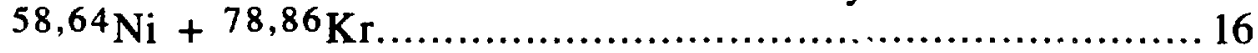

c.3. High Energy $\gamma$-Rays from ${ }^{252} \mathrm{Cf}$ Spontaneous Fission............... 17

c.4. Time-Scale in Quasi-Fission Reactions.......................... 18

c.5. Time Scale of the Fission Process...................................... 19

c.6. The General Law of Radioactive Decay Applied to Fission of Hot Nuclei ................................................... 20

c.7. Evaporation Residue Cross Sections for ${ }^{32} S+{ }^{184} W-$ Energy Dissipation in Hot Nuclei......................................... 21

c.8. Study of Proton Radioactivities.................................... 21

c.9. Alpha Decay of Heavy Nuclei ................................. 22 
a. States in the Superdeformed Secondary Minimum and Their Coupling with States in the Normal Well .................. 24

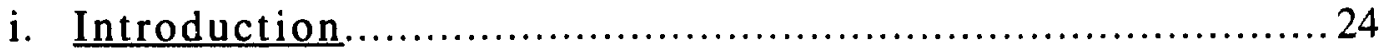

ii. Decay of SD Bands ............................................... 25

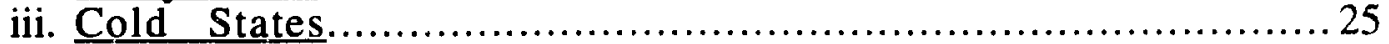

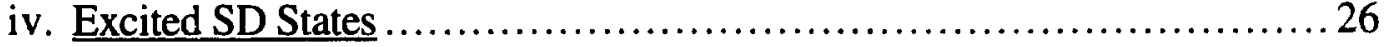

a.1. Spectrum of $\gamma$ Rays Connecting Superdeformed and Normal States in ${ }^{192} \mathrm{Hg}$................................. 26

a.2. New Techniques for Quasicontinuum Spectroscopy for Investigating the Decay Out of Superdeformed Bands .......... 28

a.3. Calculations of the Decay Out of SD Bands .................... 28

a.4. Calculation of the Spectrum of $\gamma$ Rays Connecting Superdeformed and Normally Deformed Nuclear States............ 28

a.5. Spectra of $\gamma$ Rays Feeding Superdeformed Bands ................ 29

a.6. Decay from the Superdeformed Bands in ${ }^{194} \mathrm{Hg} \ldots \ldots \ldots \ldots \ldots \ldots \ldots 29$

a.7. Moment of Inertia at the Highest Spins in ${ }^{192} \mathrm{Hg} \ldots \ldots \ldots \ldots \ldots \ldots \ldots . \ldots \ldots$

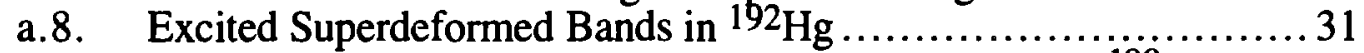

a.9. Inter-Band Coincidences in the Superdeformed Well of ${ }^{190} \mathrm{Hg} \ldots \ldots .31$

a.10. Spectroscopy of the Superdeformed Band in ${ }^{196} \mathrm{~Pb} \ldots \ldots \ldots \ldots \ldots \ldots . \ldots 33$

a.11. New Features of Superdeformed Bands in ${ }^{194} \mathrm{Hg} \ldots \ldots \ldots \ldots \ldots \ldots \ldots . \ldots 34$

a.12. Lifetime Measurement in the Superdeformed Bands of ${ }^{194} \mathrm{Hg} \ldots \ldots . .36$

a.13. Search for Excited Superdeformed Bands in ${ }^{151}$ Dy .................. 38

a.14. Search for Extremely Deformed Systems in ${ }^{182} \mathrm{Os} \ldots \ldots \ldots \ldots \ldots \ldots . \ldots . \ldots . \ldots$

b. Shape Changes in Nuclei ........................................... 39

b.1. Yrast and Near-Yrast Spectroscopy in ${ }^{188-190} \mathrm{Hg} \ldots \ldots \ldots \ldots \ldots \ldots . \ldots 40$

b.2. Collectivity of Dipole Bands in ${ }^{2} 96 \mathrm{~Pb}$..............................41

b.3. Collective and Quasiparticle Structures in ${ }^{192} \mathrm{~Pb} \ldots \ldots \ldots \ldots \ldots \ldots \ldots . \ldots 2$

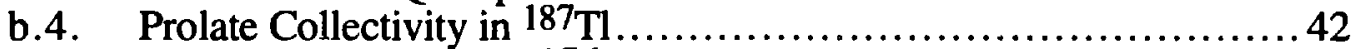

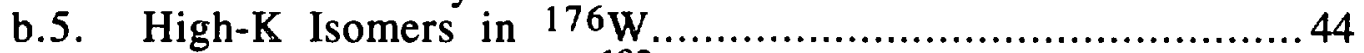

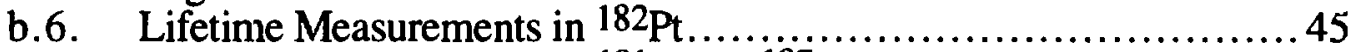

b.7. Lifetime Measurements in 181 Ir and 187 Au ............................ 45

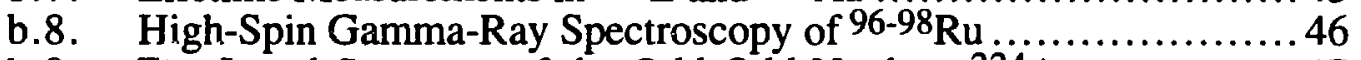

b.9. The Level Structure of the Odd-Odd Nucleus ${ }^{224} \mathrm{Ac}$.................... 47

b.10. Octupole Shapes in Nuclei ................................... 48

b.11. Lifetime Measurements in New Neutron-Rich Nd Isotopes with the Doppler Profile Method ................................... 49

b.12. The Observation of a Two-Quasi-Neutron Rotational Band in ${ }^{102} \mathrm{Zr}$...

b.13. Gamma Spectrum Following Neutron Capture in ${ }^{167} \mathrm{Er}$................ 50

b.14. Gamma-Ray Studies of Neutron-Rich Products of Deep-Inelastic Heavy-Ion Reactions...................................50

b.15. Structure Studies in the Light Hg Isotopes from Gamma-FMA Coincidence Measurements ....................55

b.16. Spectroscopy of $186 \mathrm{~Pb}$ with Mass Identification ...................552

b.17. Identification of $183 \mathrm{Hg}$ : Identical Bands in $183,185 \mathrm{Hg} \ldots \ldots \ldots \ldots \ldots 53$

b. 18. FMA Studies of Proton-Rich Nuclei $N \sim 82$ by Gamma-ray and Conversion Electron Spectroscopy.................... 55 
C. ACCELERATOR MASS SPECTROMETRY (AMS) AND EXPERIMENTS WITH SECONDARY BEAMS ....................... 56

a. Detection of ${ }^{81} \mathrm{Kr}$ with Accelerator Mass Spectrometry ...................... 56

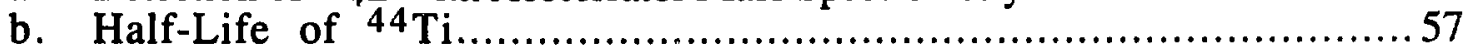

c. Secondary Beam Development with Inverse Kinematic Reactions........... 57

d. Development Studies for an Experiment with a ${ }^{18} \mathrm{~F}$ Beam ................... 58

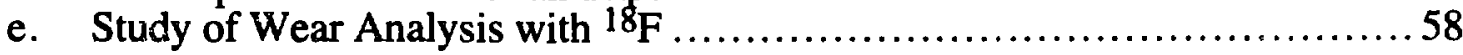

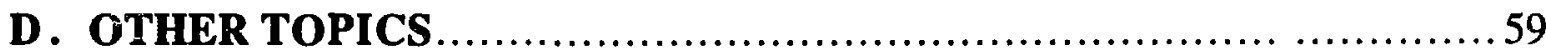

a. Measurements with Laser-Cooled Beams at ASTRID ....................... 59

b. Properties of Crystalline Ions ............................................ 59

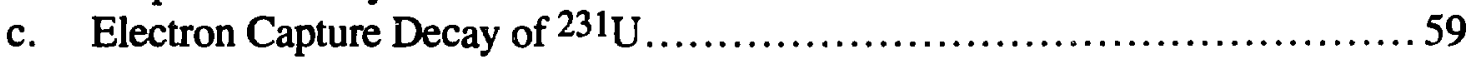

E. EQUIPMENT DEVELOPMENT AT THE ATLAS FACILITY ............60

a. Fragment Mass Analyzer Project ......................................60 60

b. The ATLAS Positron Experiment (APEX) ................................... 61

c. GAMMASPHERE Activities at Argonne..................................... 64

c. 1. Testing and Procurement of the BGO Compton-

Suppression Detectors ...........................................65

c. 2. Target Chamber for GAMMASPHERE .............................65

c.3. GAMMASPHERE Software Development...........................66 66

c.4. Software for Analyzing High-Fold Coincidence Data......................66

d. Status of the Argonne Notre Dame BGO Gamma-Ray

Facility at ATLAS ....................................................6 67

e. A Particle Ball for GAMMASPHERE .....................................6 68

f. The Study of Fusion Reactions with a Gas-Filled Magnetic Spectrograph .............................................6 68

g. Study of the Particle Identification for Low-Z Ions at Energies

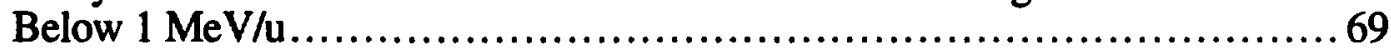

h. Radiation Safety at Low-Energy Heavy-Ion Accelerators .....................69 69

i. Physics Division Computer Facilities.................................. 69

j. Data-Acquisition Systems ............................................. 70

k. Nuclear Target Development.............................................. 70

F. ASSISTANCE TO OUTSIDE USERS OF ATLAS..................73

a. Experiments Involving Outside Users ...................................74

b. Outside Users of ATLAS and of ATLAS Technology During the

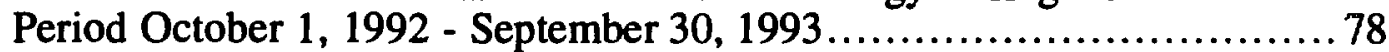

c. Summaries of the Continuing User Programs for FY 1993.......................80

c.a.1. Nuclear Physics ...................................................... 80

c.a.2. Atomic Physics ..................................................... 80

d. ATLAS - Technology Transfer ................................... 82 
A. OPERATION OF THE ACCELERATOR ........................... 86

B . RECENT AND PLANNED IMPROVEMENTS AT ATLAS ............88

a. Upgrade of the Linac Control System ................................ 88

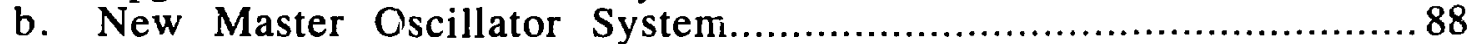

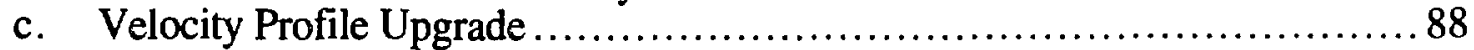

d. Helium-Refrigeration System ...................................... 89

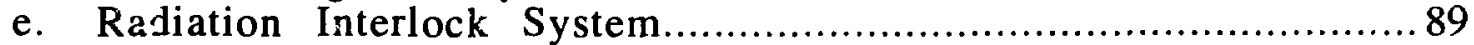

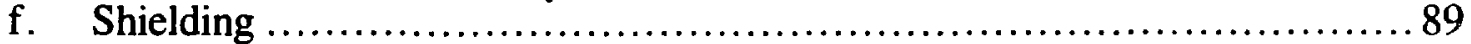

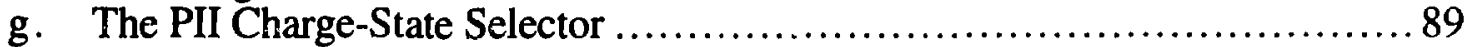

$\mathrm{h} . \quad$ The Advanced ECR Project ........................................ 90

C. ACCELERATOR PHYSICS AND LINAC DEVELOPMENT ...........93

a. Status of the Positive-Ion Injector ....................................... 93

b. ECR Source and Voltage Platform ................................... 94

c. PII Beam Tuning ................................................... 94

d. Positive-Ion Injector and ATLAS Linac ............................... 94

e. Beam Diagnostics ................................................. 95

f. Technology of RF Superconductivity......................................... 95

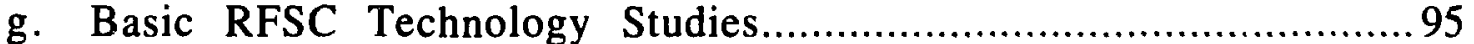

h. Beam Dynamics and Alternating Phase Focusing ....................... 95

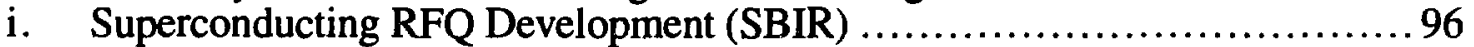

j. Superconducting Resonators for the New Delhi Booster Linac ................96

Radioactive Beam Initiatives ......................................... 96

k. Accelerator Concepts for the Front End .............................. 96

$\ell$. Electron Beam Ionizer .............................................. 96

III. MEDIUM-ENERGY NUCLEAR PHYSICS RESEARCH.........97

A. SUBNUCLEONIC EFFECTS IN NUCLEI ...........................98

a. Deep-Inelastic Muon Scattering from Nuclei with Hadron Detection .......... 98

b. Electron-Deuteron Scattering With a Polarized Deuterium Gas

Target in the VEPP-3 Electron Storage Ring.......................................10

c. Laser-Driven Polarized Deuterium Gas Target....................................101

d. Two-Body Photodisintegration of the Deuteron at High Energy: Experiment NE17 at SLAC ...........................................102

e. Nuclear and $Q^{2}$ Dependence of Quasielastic (e,e'p) Scattering at Large Momentum Transfer..............................................103

f. Measurement of the Helicity-Dependent Asymmetry in ${ }^{3} \overrightarrow{\mathrm{He}}\left(\overrightarrow{\mathrm{e}}, \mathrm{e}^{\prime}\right)$ Quasielastic Scattering ........................................................104

g. Investigation of the ${ }^{3} \mathrm{He}$ Wave Function by Quasifree Scattering ..............105

h. Studies of Particle Production in Collisions of Relativistic Heavy Ions with iNuclei........................................................... 105

i. Electroproduction of Kaons and Light Hypernuclei..................................106

j. Two-Body Photodisintegration of the Deuteron at Forward Angles and Photon Energies Between 1.5 and $4.0 \mathrm{GeV}$. 
k. A Study of Longitudinal Charged-Pion Electroproduction in D,

${ }^{3} \mathrm{He}$, and ${ }^{4} \mathrm{He}$.

$\ell$. The Energy Dependence of Nucleon Propagation in Nuclei as

Measured in the (e,e'p) Reaction.............................................. 107

m. Measurement of Proton Polarization in the $\mathrm{d}(\gamma, \mathrm{p})$ n Reaction............... 108

n. Short-Orbit Spectrometer for Hall C........................................... 108

o. Proposal to Measure Spin-Structure Functions and Semi-Exclusive

Asymmetries for the Proton and Neutron at HERA ...................... 110

p. Measurement of $\bar{d} / \bar{u}$ in the Nucleon .................................. 111

IV. THEORETICAL PHYSICS

A. NUCLEAR DYNAMICS WITH SUB-NUCLEONIC DEGREES OF FREEDOM

a. Hadronic and Electromagnetic Production of Mesons and

Nucleon Resonances at GeV Energies ................................ 114

b. Chiral Symmetry and Electroproduction of Pions at Threshold in Chiral Perturbation Theory ......................................... 114

c. Short-Range Exchange Contributions to Pion Absorption

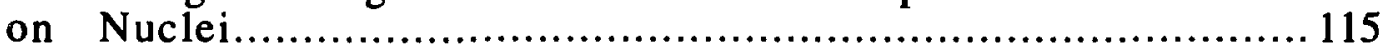

d. Dependence of $\mathrm{p}\left(\mathrm{e}, \mathrm{e}^{\prime} \pi\right)$ Cross Sections on the $\gamma \mathrm{N} \leftrightarrow \Delta$ Transition Form Factors ............................................ 115

e. Study of $\left(e, e^{\prime}\right),\left(\pi, \pi^{\prime}\right)$ and $(\gamma, \pi)$ Reactions on Nuclei ................... 115

f. Electroproduction of Pions on the Deuteron and Three-Nucleon

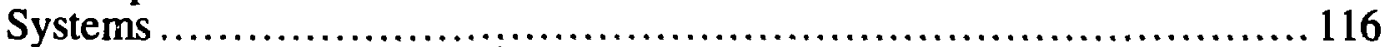

g. Spin Structure Functions of ${ }^{3} \mathrm{He}$ and the Deuteron ....................... 116

h. The Electromagnetic Current Operator and the Blankenbecler-Sugar

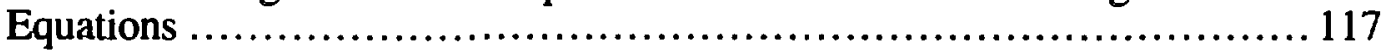

i. Nontrivial Vacuum Structure in Light-Front Hamiltonian Dynamics of Infinite Systems.......................................................... 117

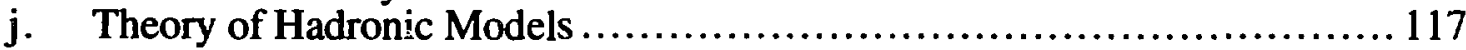

k. Gauge Covariance and the Fermion-Photon Vertex in Threeand Four- Dimensional, Massless Quantum Electrodynamics................ 118

$\ell$. $\pi-\pi$ Scattering in a QCD-Based Model Field Theory ...................... 118

m. $\rho \pi \pi$ and $\omega \pi \pi$ Vertex Functions Within a Confined-Quark Model.............. 119

n. $\rho-\omega$ Mixing Self-Energy and Model Quark-Gluon Dynamics ...............120

o. Pion Loop Contribution to the Electromagnetic Pion Charge Radius........... 120

p. Electromagnetic Pion Form Factor............................................. 120

q. Dynamical Chiral Symmetry Breaking and Confinement With an Infrared-Vanishing Gluon Propagator ............................... 121

r. Dyson-Schwinger Equations and Their Application to Hadronic Physics ............................................................ 121

s. Complex Singularities in the Quark Propagator......................... 121

t. Dyson-Schwinger Equation Study of the Effect of the HiggsMechanism on Dynamical Chiral Symmetry Breaking .................... 122

u. QCD at Finite Temperature and Density....................................... 122

v. Medium Effects on $\Delta$-Propagation, $\mathrm{NN}$ and $\mathrm{N} \Delta$ Collisions.................... 122

w. Effective Potentials for an Interacting Hadron Gas ...................... 123

$\mathrm{x}$. Excitation of Nucleon Resonances............................................ 123

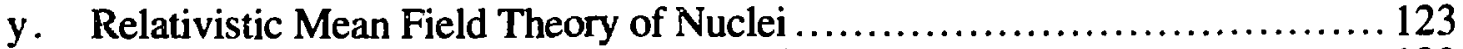

z. Topological Effects in Quantum Mechanics .......................... 123 
a. New Argonne Nucleon-Nucleon Potential ............................ 125

b. Variational and Green's Function Monte Carlo Calculations of Few-Body Nuclear Ground States ................................. 126

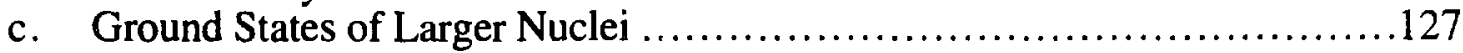

d. Nucleon, Nucleon-Cluster, $\Delta$-Isobar and Excess Pion

Momentum Distributions in Finite Nuslei ..............................127

e. Coulomb Sum and Proton-Proton Correlations in Nuclei....................128

f. Monte Carlo Calculations of (e,e'p) Reactions ..........................129

g. Isovector Spin-Longitudinal and -Transverse Response of Nuclei.............129

$\mathrm{h} . \quad$ Nuclear and Neutron Matter Studies .....................................130

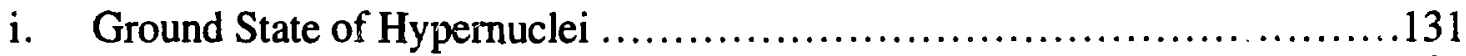

j. $\quad \Lambda$ Single-Particle Energies.................................................131

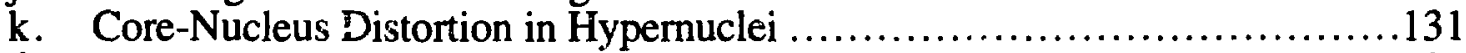

$\ell$. Space-Exchange Effects in Light Hypernuclei ..........................132

$\mathrm{m}$. Charge-Symmetry Breaking $\Lambda$-Nucleon Interaction ....................132

$n$. Suppression of the $\Lambda-\Sigma$ Coupling in Nuclear Matter...........................132

C. HEAVY-ION REACTIONS ............................................

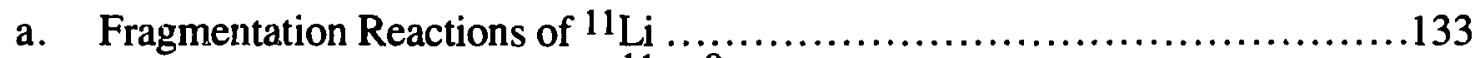

b. Momentum Distributions for $\left({ }^{1}{ }^{1} \mathrm{Li}, 9 \mathrm{Li}+\mathrm{n}+\mathrm{n}\right)$ Three-Body

Breakup Reactions ....................................................133

c. Momentum Distributions for Nuclear Induced Brealsup

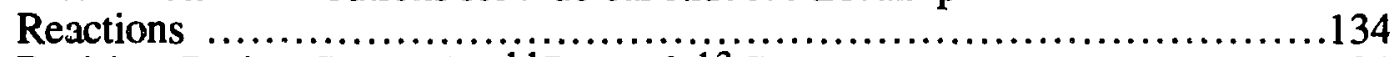

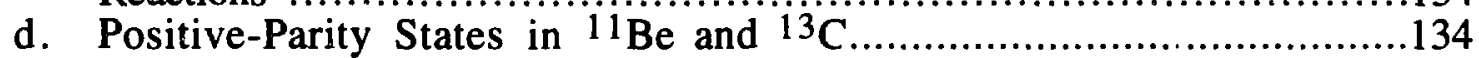

e. Heavy-iun Reactions near the Coulomb Barrier ........................134

f. Spin Distributions in Heavy-Ion Fusion Reactions ...............................135

D. NUCLEAR STRUCTURE STUDIES ................................

a. Configuration Interaction Effects in Rotational Bands of

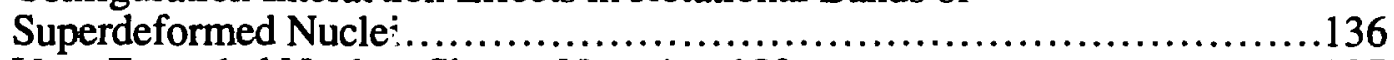

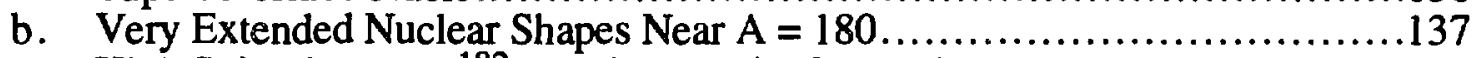

c. High Spin Shapes in ${ }^{182}$ Os With Density-Dependent

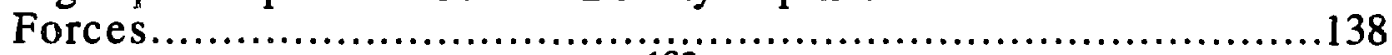

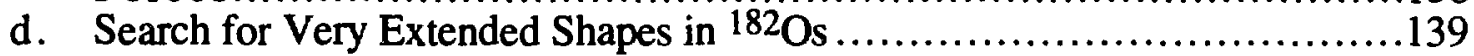

e. Single-Particle States in the Heaviest Elements...............................139

f. Exploration of Nuclear Energy Surfaces at High Spin in Multi-Dimensional Deformation Spaces ....................................139

g. Many-Body Wave Functions ....................................... 140

$h$. Rotation of Thermally Excited Nuclei .................................. 140

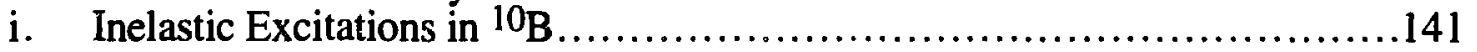

E. ATOMIC PHYSICS .....................................................

a. Isotope Sputtering ............................................... 142

b. The Mean Ion Charge in Ar Photoionization near the K Edge ................142

c. Double Electron Ionization in Compton Scattering of High

Energy Photons by Helium Atoms.......................................142

d. Ionization of Atoms by High-Energy Photons...................................142 

a. Forbidden Transitions in Few-Electron Ions
b. The Fragmentation of Fullerenes by Fast Heavy Ions.
c. Precision Spectroscopy of the $2 s-2 p$ Fine Structure
Transitions in Helium-like $\mathrm{Ar}^{16+}$ and $\mathrm{N}: 26+$.
d. Measurement of Transition Energies in Li-Like and He-Like Calcium.................................................... 148
e. RTE and REC in Collisions of $\mathrm{U}^{90+}$ Ions on Carbon..................... 148

B. FAST-ION-BEAM/LASER STUDIES AT BLASE...................... 149

a. Precision Lifetime Measurements by Single-Photon Counting................. 149

b. Hyperfine Structure Studies of Zr II............................................151

c. Laser-rf Double Resonance Studies of Hyperfine Structure in V II .......... 151

d. Hyperfine Structure Studies in Nb II ................................ 152

e. Precision Lifetime Measurements of the $6 \mathrm{p}$ Levels in Cesium ............... 152

f. Precision Lifetime Measurements of the $2 p$ Levels in Lithium .............. 153

C. COULOMB-EXPLOSION EXPERIMENTS ........................ 153

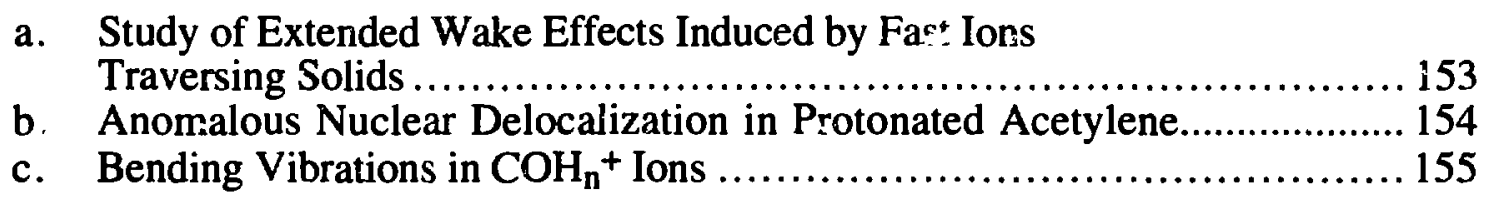

D. ATOMIC PHYSICS AT SYNCHROTRON LIGHT SOURCES ....... 156

a. X-Ray Absorption Spectroscopy of Inner-Shell Double

Photoionization in Argon ......................................... 156

b. The X-Ray Photoabsorption Spectrum of Potassium and

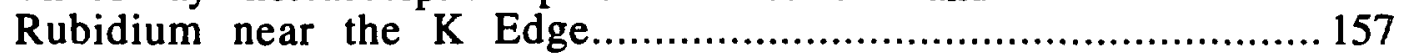

c. Photoabsorption in Helium at 2- to 14-keV Photon Energies ............... 158

d. Absolute Photoabsorption Cross Section in Argon near the K Edge ......................................................... 158

e. Time-of-Flight Measurements of Ion Production Rates from Photoionization of Potassium and Argon near the K Edge .................. 159

f. X-Ray Resonant Raman Spectroscopy (XRRS) ....................... 159

g. Auger Resonant Raman Spectroscopy in Argon .......................... 159

h. Satellite Structure in the Argon 1s Photoelectron Spectrum .................. 159

i. Planning for the Basic Energy Sciences Radiation Center

(BESSRC) and other Facilities at the Advanced Photon Source ............. 160

E. ACCELERATOR FACILITIES FOR ATOMIC PHYSICS ............. 160

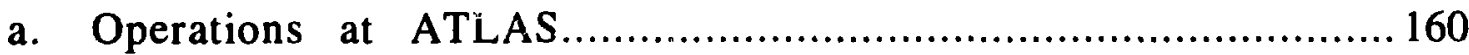

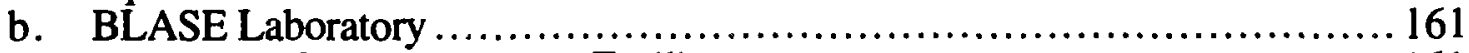

c. Operation of the Dynamitron Facility ................................. 161 
OTHER EDUCATIONAL ACTIVITIES IN THE PHYSICS DIVISION ..163

a. Enhancement of Minority Involvement in DOE Nuclear

Physics Programs

b. Scientific Support of SciTech Museum Exhibits and

Outreach Programs. $16 \overline{3}$

c. Exhibit Development. 164

Staff List

Publications 


\section{HEAVY-ION NUCLEAR PHYSICS RESEARCH}

The heavy ion research program in the Argonne Physics Division spans a wide range of studies from quasi-elastic reactions to the search for nuclei far away frcm the valley of stability and the search for exotic new particles in collisions hetween very heavy nuclei. This is a research prograni with involvement from many university groups anc sutside institutions, characterized by extensive collaborations between in-house and outside researchers. Most of these studies are carried out at the ATLAS accelerator, but parts of the program take advantage of forefront instrumentation available elsewhere, in particular GAMMASPHERE, EUROGAM, and radioactive beams.

A major part of the research effort is devoted to $\gamma$-ray experiments with a focus on studics of superdeformation (SD). This research addresses the physics associated with states within the SD well and their coupling with states outside the well. Although states near the minimum of the SD well may lie high above the yrast line, they are cold since they are isolated from the hot normal states by a potential barrier.

A new "island" of superdeformation in the mass 190 region was first found a few years ago in experiments at ATLAS. Since then many more SD bands have been reported in this region. This large body of data was vital in identifying the occurrence of several pairs of "identical" bands. The identical bands, which were not anticipated, are still not explained, and may imply a symmetry which has yet to be identified. Recently, in an experiment at GAMMASPHERE, a band in ${ }^{151}$ Dy was found vith energies midway between those in ${ }^{152} \mathrm{Dy}$. This provides additional evidence for the validity of a pseudospin coupling scheme first proposed by Nazarewicz et al. to account for the identical band phenomenon near $A=150$. In experiments performed with the early implementation phase of GAMMASPHERE, first indications for an octupole vibrational SD band in ${ }^{190} \mathrm{Hg}$ were discovered at a surpi isingly low energy $(\sim 600 \mathrm{keV})$ above the yrast $S D$ band.

A new approach to study the strikingly sudden decay of the SD band has been devised by the Argonne group. By observing the complete spectrum of $\gamma$-rays characterizing this decay a quasicontinuous distribution was observed with superimposed broad structures as well as sharp peak: This shows that the decay originates from the coupling between the SD states witn the sea of normal states in which they are embedded, providing a case study for the coupling of a cold ordered system into a hot chaotic one. From EUROGAM and GAMMASPHERE data it was established that SD bands give rise to a pronounced E2 bump in the $\gamma$ spectrum, a feature which allows to probe the collective properties of excited SD states. From simulations one can begin to understand the large population of SD states, and set constraints on the excitation energy of the band above the yrast line as well as the depth of the SD well.

With the advent of the new detector arrays the emphasis of structure studies at ATLAS has shifted towards nuclei which are difficult to investigate with conventional techniques. The combination of the Argonne Notre Dame BGO $\gamma$-ray facility with the Fragment Mass Analyzer (FMA) has proven to be very successful. For example, studies of the ${ }^{183} \mathrm{Hg}$ and ${ }^{186} \mathrm{~Pb}$ nuclei with mass identification have uncovered new cases of i.jentical bands.

A second major rasearch program is the study of exotic nuclei far from the valley of beta stability. The high-quality, high-intensity heavy ion beams from ATLAS and the high detection efficiency and excellent background suppression of the FMA provide unique research opportunities ranging from proton radioactivity of heavy nuclei at the proton drip line to the production of isotopes of the heaviest elements and the study of their structure.

The separation of these products of interest from background has been improved with the recently installed recoil implantation system at the FMA. Nuclei identified according to their mass in the focal plane of the FMA are implanted in a Si-detector (segmented into 2304 pixels) located behind 
the focal plane detector. A subsequent alpha or proton-decay at the implantation site, correlated with time-of-flight and recoil energy information, results in drastic background reduction and cross sections at the nanobarn and sub-nanobarn level can be measured. Experiments have been performed with the goal of extending proton drip line studies towards lighter $\left({ }^{109} \mathrm{I},{ }^{105} \mathrm{Sb}\right)$ and heavier $\left({ }^{156} \mathrm{Ta},{ }^{160} \mathrm{Re}\right)$ nuclei and experiments for higher- $Z$ nuclei have begun. The implantation system also opens new opportunities for the study of very heavy nuclei. First experiments studying the alpha decays in the systems ${ }^{58} \mathrm{Ni}+{ }^{116} \mathrm{Sn}\left(\mathrm{Z}_{\text {total }}=78\right),{ }^{92} \mathrm{Mo}+{ }^{92} \mathrm{Mo}\left(\mathrm{Z}_{\text {total }}=84\right)$, ${ }^{64} \mathrm{Ni} \therefore{ }^{54} \mathrm{Sm}\left(Z_{\text {iotal }}=90\right)$, and ${ }^{64} \mathrm{Ni}+{ }^{181} \mathrm{Ta}\left(Z_{\text {total }}=101\right)$ have been performed.

The ATLAS Positron Experiment (APEX) studies the effects of strong electromagnetic fields in close collisions of high-Z atoms at energies close to the Coulomb barrier. This situation has been predicted to give rise to qualitatively new phenomena associated with the over-critical binding of the inner electron orbits, such as the spontaneous emission of positrons. Experiments originally motivated by these ideas, carried out at GSI Darmstadt over the past decade, have produced some remarkable and unusual results which, if confirmed, would seem to signal the appearance of some interesting fundamental new physics.

APEX is a second-generation experiment, specifically designed to answer these questions. The apparatus has been designed and constructed by a collaboration of scientists from Argonne, Chicago, Florida State, Michigan State, Princeton, Queen's, Rochester, Washington and Yale. The experiment features a high-acceptance, high-resolution detection system which, coupled with the high-intensity, $100 \%$ duty factor beams from the ATLAS accelerator allows data rates above those of the first generation experiments and also allows, for the first time, a direct determination of the angles of emission of the positrons and electrons.

The apparatus has recently been compieted and, over the past year, has progressed from the testing phase to full operation. Initial experience has shown that, in essentially all respects, the apparatus performs according to the design specifications. Beams of $238 \mathrm{U}$ of intensity up to 5 pnA from ATLAS have been used to bombard $1 \mathrm{mg} / \mathrm{cm}^{2} 181 \mathrm{Ta}$ targets and beam induced positrons have been measured with high resoluticn. In the first physics production run, which took place in December 1993, a total of $1,000,000$ positrons were measured with over 400,000 positron-electron coincidence events. These data are currently being analyzed. It is already clear that the first operation of the complete device has resulted in a significant data set.

Nuclear reactions at energies close to the Coulomb barrier, where relative velocities between interacting nuclei are smail, provide a unique regime for the study and understanding of the aissociated dynamics. Studies of the systems $59,64 \mathrm{Ni}+92,100 \mathrm{Mo}$ by measurements of the quasielastic transfer reactions at sub-barrier energies have continued. In these experiments the cross sections for up to six-neutron transfer reactions have been determined unambiguously for the first time. In collaboration with the theory group at Argonne a consistent coupled channels description of all the reaction channeỉs (transfer and fusion) in these systems has been achieved. Recent measurements of fusion cross sections in the system $\mathrm{Ni}+\mathrm{Kr}$ using the gas-filled magnet technique for particle separation indicate very large, and as yet unexplained, sub-barrier fusion enhancements in reactions involving ${ }^{78} \mathrm{Kr}$, a nucleus with high polarizability.

In ${ }^{24} \mathrm{Mg}$, very deformed, chain-like configurations of six $\alpha$ particles are suggested by calculations of the potential energy surface to be sufficiently stable that they could be observed as resonances in the appropriate reaction channels. Measurements for ${ }^{12} \mathrm{C}+{ }^{12} \mathrm{C}$ inelastic scattering have continued using a highly segmented detector system of double-sided $\mathrm{Si}$ strip detectors. The reaction

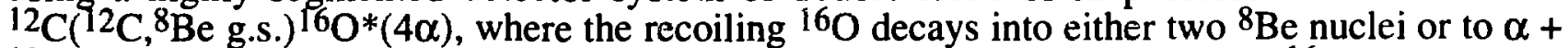
${ }^{12} \mathrm{C}\left(\mathrm{O}^{+} 2\right)$ has conclusively been identified. Discrete levels were observed in $16 \mathrm{O}$ populated at excitation energies near 17 and $19 \mathrm{MeV}$, in the same range of energies where strong resonance behavior in $\alpha+{ }^{12} \mathrm{C}$ scattering has been interpreted as reflecting the population of elongated alphaparticle configurations in 160 . 
Dynamics of fission processes offer a way to explore the influence of energy dissipation and mass flow in a strongly deformed heavy system. Giant Dipole Resonance (GDR) $\gamma$-rays emitted during the fission process itself have been measured in collaboration with SUNY at Stony Brook. At high excitation encrgies, these results appear to be well described by the one-body dissipation model, but at lower excitation energies the dissipation is strongly reduced. This observation will be studied further in measurements at ATLAS of pre-scission neutrons and evaporation residue cross sections with the aim of obtaining a better theoretical understanding of the nuclear dissipation against large scale deformations at moderate excitation energies.

Accelerator Mass Spectrometry (AMS) remains an active program taking advantage of particular features of ATLAS such as good stability, high transmission, low beam backgrounds. This research program places particular emphasis on heavy radioisotopes which cannot be studied elsewhere. After the successful detection of $269-\mathrm{yr}^{39} \mathrm{Ar}$ at natural levels at ATLAS, a series of experiments studying AMS with $210,000 \mathrm{yr}{ }^{81} \mathrm{Kr}$ has been initiated. These experiments were performed both at ATLAS and at the A1200 recoil separator at MSU.

Experiments in the areas of astrophysics and applied physics utilizing some unique features of secondary beams have recently started. The first studies will use the $1.8 \cdot \mathrm{hr}{ }^{18}{ }^{18}$ isotope. For an astrophysical experiment studying the transition from the hot $\mathrm{CNO}$ cycle to the rp-process, ${ }^{18 F}$ will be produced at a high-current cyciotron and the radioisotope $18 \mathrm{~F}$ will be transferred in the negativeion source of the tandem. For a study of the wear-analysis of various industrial components, ${ }^{18} \mathrm{~F}$ will be produced at ATLAS via the $\mathrm{p}\left({ }^{18} \mathrm{O},{ }^{18} \mathrm{~F}\right) \mathrm{n}$ reaction and implanted into the material of interest. Improvements in the sensitivity for wear-analysis by a factor of 100 compared to previous techniques are expected.

\section{A. REACTION STUDIES}

Reaction studies at ATLAS range from quasi-elastic reactions to more complex fusion and fission processes, addressing connections between reaction dynamics and structure. The good beam quality and easy energy variability make ATLAS very well suited for these studies. The res:arch continues to focus on three major areas: (a) the study of nuclear reaction mechanisms at energies in the vicinity of the Coulomb barrier, (b) studies of the production and decay of exotic nuclear cluster states in s-d-shell nuclei, and (c) the production of nuclei at the limits of stability. New techniques have been developed to improve the efficiency and separation capabilities for these measurements. The Fragment Mass Analyzer (FMA) with its excellent mass resolution and high background suppression was used successfully in a series of measurements probing nuclei in the vicinity of the proton drip line. The multi-particle detector arrays of Si strip detectors have been upgraded to improve the efficiency for the detection of six-alpha decay channels. In sub-barrier fusion experiments, the gas-filled magnet was employed successfully for the study of sub-barrier fusion enhancement involving target nuclei in the mass-90 region.

\section{a. Quasi-Elastic and Deep-Inelastic Reactions Close to the Coulomb Barrier}

Nuclear reactions at energies close to the barrier, where relative velocities between interacting nuclei are small, provide a unique regime for the study and understanding of reaction dynamics. Our previous work showed that quasi-elastic reactions are the dominant processes at energies below the barrier and can strongly influence other reaction modes. The experiments in the last year concentrated on the system $58 \mathrm{Ni}+98,100 \mathrm{Mo}$ where fusion excitation functions for $58,64 \mathrm{Ni}+$ $92,100 \mathrm{Mo}$ were measured previously. Similar to the system $58,64 \mathrm{Ni}+58,64 \mathrm{Ni}$, a correlation was found between the strength of the quasi-elastic yields and the deviation of the measured fusion cross sections from the corresponding coupled-channel calculations. For the system ${ }^{58} \mathrm{Ni}+{ }^{100} \mathrm{Mo}$, which shows the strongest transfer yields, a chain of neutron transfer reactions ranging from the In to the $6 \mathrm{n}$ transfer were detected for the first time. Intense molybdenum beams from the positive-ion injector and the use of inverse reaction kinematics were crucial in these experiments. 
Deep-inelastic reactions were studied in the system ${ }^{64} \mathrm{Ni}+{ }^{136} \mathrm{Xe}$ in a kinematic coincidence experiment. The energy dependence and the yields for the deep-inelastic processes were found to be very similar to measured previously in the system ${ }^{58} \mathrm{Ni}+{ }^{124} \mathrm{Sn}$. This indicates that these reactions, which at low energies cannot be described in simple friction-type models, seem to be a universal phenomenon in heavy systems.

\section{a.1. Observation of One- To Six-Neutron Transfer Reactions (C. L. Jiang, K. E. Rehm, J. Gehring, B. Glagola, W. Kutschera, M. Rhein, and A. H. Wuosmaa)}

Several theoretical papers investigated the possibility of observing the transfur of several correlated pairs of nucleons through the contact surface between two nuclei during grazing collisions. Due to interference effects between different transfer amplitudes (and neglecting abs $r_{i}^{\prime}$ ion), oscillations in the cross sections for multi-pair transfier processes have been predicted. Because of the strong influence of the absorptive potential on these transfer processes, multi-nucleon transfer reactions should be studied at energies close to the Coulomb barrier.

We investigated multi-neutron-pair transfer reactions in the system ${ }^{58} \mathrm{Ni}+{ }^{100} \mathrm{Mo}$ using highlyenriched ${ }^{58} \mathrm{Ni}$ targets and a ${ }^{100} \mathrm{Mo}$ beam from the positive-ion injector at ATLAS. Ining the advantages of the inverse reaction kinematics, the target-like reaction products are emitted at forward angles with energies that allow us to determine uniquely their mass and charge. The particles were momentum-analyzed in the split-pole spectrograph and identified in the focal plane with a system consisting of a hybrid position-sensitive, parallel-plate, avalanche - Bragg-Curve detector.

The bombarding energies ranged from $\mathrm{E}_{\mathrm{cm}}=117-137 \mathrm{MeV}$. The Coulomb barrier, extracted from the systematics of heavy-ion-induced fusion cross sections, is about $V_{c}=143 \mathrm{MeV}$. At the highest bombarding energy of $137 \mathrm{MeV}$, Ni-recoils in the mass range $\mathrm{A}=57-64$ were observed (Fig. I-1). With the exception of $A=58$, all recoil products exhibit angular distributions that are backward peaked. The yields for these multi-particle transfer reactions decrease by about a factor of 3-4 for each transferred neutron. Theoretical calculations predict about a factor-of-10 decrease in yield per each transferred neutron pair, in good agreement with the experimental results.

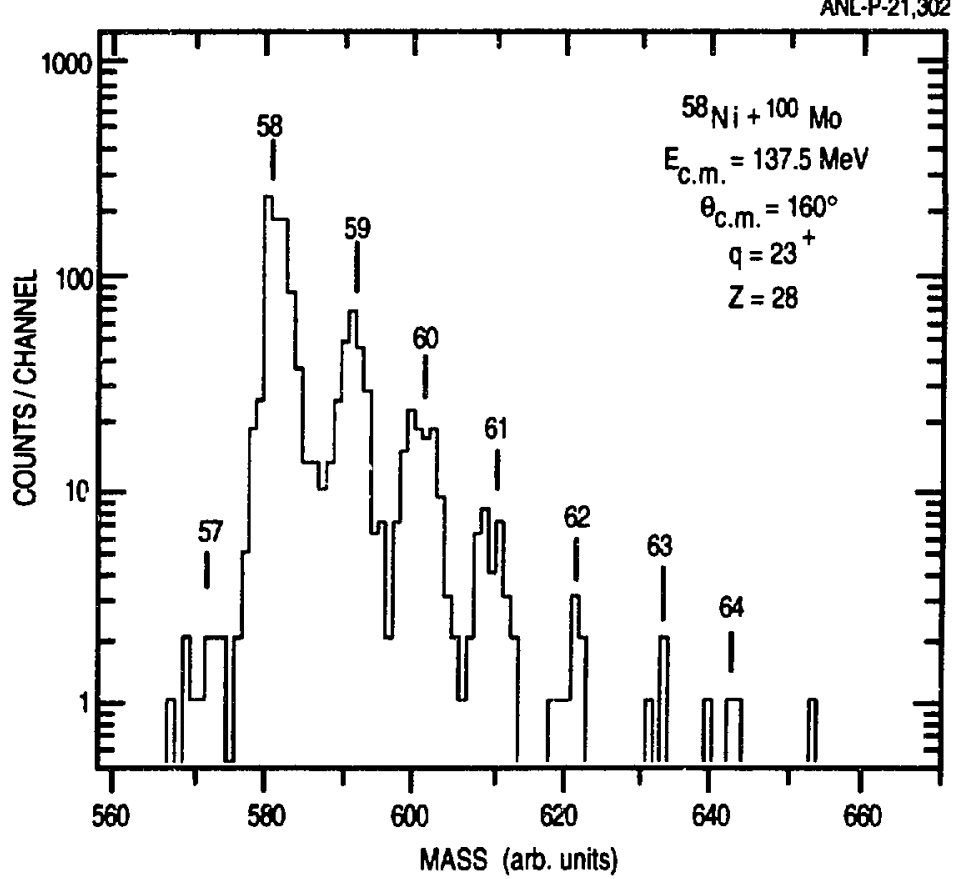

Fig. 1-1. Mass spectrum for particles with $Z=28$ and charge state $q=23^{+}$measured for the system ${ }^{58} \mathrm{Ni}+100 \mathrm{Mo}$ at $\theta_{\text {lab }}=10^{\circ}$ and $E_{c . m}=137.5 \mathrm{MeV}$. 


\section{a.2. The Influence of Transfer Reactions on the Sub-Barrier Fusion \\ Enhancement in the Systems $58,64 \mathbf{N i}+{ }^{92,100} \mathrm{Mo}$ (K. E. Rehm, C. L. Jiang, H. Esbensen, J. Gehring, B. Glagola, W. Kutschera, Y. Liang, M. Rhein, K. Teh, F. L. H. Wolfs, and A. H. Wuosmaa)}

Most of the measurements of the sub-barrier fusion enhancement in the systems $58,64 \mathrm{Ni}+$ 92,100 Mo can be explained by coupled-channels calculations, which include the collective excitations of one- and two-phonon states in projectile and target. However, discrepancies

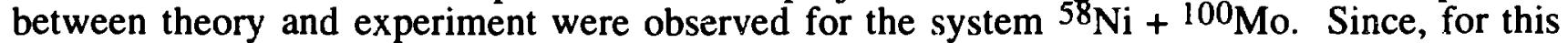
system, positive ground-state Q-values for neutron-transfer reactions are available, we began a study of transfer strengths in the $\mathrm{Ni}+\mathrm{Mo}$ systems at energies in the vicinity of the Coulomb barrier. The experiments were done with inverse reaction kinematics using the split-pole spectrograph with its hybrid focal-plane detector for particle identification. The experiments with $100 \mathrm{Mo}$ beams on $58,64 \mathrm{Ni}$ targets have been analyzed. It was foind that the quasi-elastic transfer yields for ${ }^{58} \mathrm{Ni}+{ }^{100} \mathrm{Mo}$ are about a factor of three lower than in the ${ }^{64} \mathrm{Ni}+{ }^{100 \mathrm{Mo}}$ system. This behavior is similar to the $58,64 \mathrm{Ni}+58,64 \mathrm{Ni}$ system where a larger sub-barrier fusion enhancement in ${ }^{58} \mathrm{Ni}+{ }^{64} \mathrm{Ni}$ is correlated with a stronger transfer yield. Coupled-channel calculations including the transfer channels are presently being performed and a study of the ${ }^{92} \mathrm{Mo}+58,64 \mathrm{Ni}$ systems is planned for the near future.

\section{a.3. Energy Dependence of Neutron-Transfer Reactions in the Systems 98,100 Mo $+{ }^{58}$ Ni Below the Coulomb Barrier (C. L. Jiang, K. E. Rehm, J. Gehring, B. Glagola, W. Kutschera, Y. Liang, M. Rhein, K. Teh, and A. H. Wuosmaa)}

At bombarding energies below the Coulomb barrier the transfer of nucleons between two interacting nuclei occurs via a tunneling process resulting in an exponential eriergy dependence of the transfer cross sections. The slope parameter for this energy dependence depends on the mass and the binding energy of the transferred particle (or cluster) and should be about twice as steep for the two-neutron transfer reaction if compared to the one-neutron case. We studied these predictions for one- and two-neutron transfer reactions in the systems $98,100 \mathrm{Mo}+58 \mathrm{Ni}$ at energies below the Coulomb barrier.

The experiment was performed with ${ }^{58} \mathrm{Ni}$ targets and $98,100 \mathrm{Mo}$ beams from the positive-ion injector at ATLAS. As a detection system the split-pole spectrograph with its heavy-ion focal plane detector was used which allowed for single mass and Z-resolution up to $A=100$. The high energies of the Ni-like recoil products in inverse reaction kinematics simplified greatly the particle identification procedure at bombarding energies below the Coulomb barrier.

Because of the low incident energies, the angular distributions for the systems are all peaked at angles $\theta>100^{\circ}$. Converting the scattering angles to distances of closest approach, assuming Cu!llomb trajectories exponential fall-offs for the transfer, probabilities are observed which are independent of the bonbarding energy. The slope parameter for the one-neutron transfer reactions are in excellent agreement with the theoretical values calculated from the respective binding energies. Despite the low bombarding energies which should justify the use of semi-classical descriptions of these reactions, the slope parameters for the two-neutron transfer reactions $(58 \mathrm{Ni}$, $60 \mathrm{Ni}$ ) are only about $10-20 \%$ larger than the one-neutron values, whereas a factor-of-two increase was expected. This observation is confirmed from an analysis of the angle-integrated cross section. We are presently extracting the shape of the transfer form factors needed to describe the measured angular distributions for later comparisons with theoretical calculations. 


\section{a.4. Neutron Transfer at Large Distances in Spherical and Deformed Systems}

(K. E. Rehm, B. G. Glagola, W. Kutschera, F. L. H. Wolfs, and A. H. Wuosmaa)

Quasi-elastic few-nucleon transfer reactions induced by heavy ions are a sensitive probe of the tail of the wave functions of the transferred particles. The transfer probability, as a function of the distance of closest approach, should fall off exponentially with a decay constant $2 \alpha$ that can be calculated irom the binding energy of the transferred particle or cluster. This was studied for a variety of reactions involving spherical and deformed nuclei. The experiments were performed at the split-pole spectrograph with a focal-plane detector which allowed a complete separation of the reaction products according to their mass and charge. The majority of one-neutron transfer reactions can be well described by the simple tunneling model. The transfer probabilities for twoneutron transfer reactions, however, show deviations from the semi-classical predictions with a disagreement that increases at higher bombarding energies (Fig. I-2). These deviations can be explained by the more localized form factors for multiparticle transfer reactions which require an analysis of the data within the diffraction model.

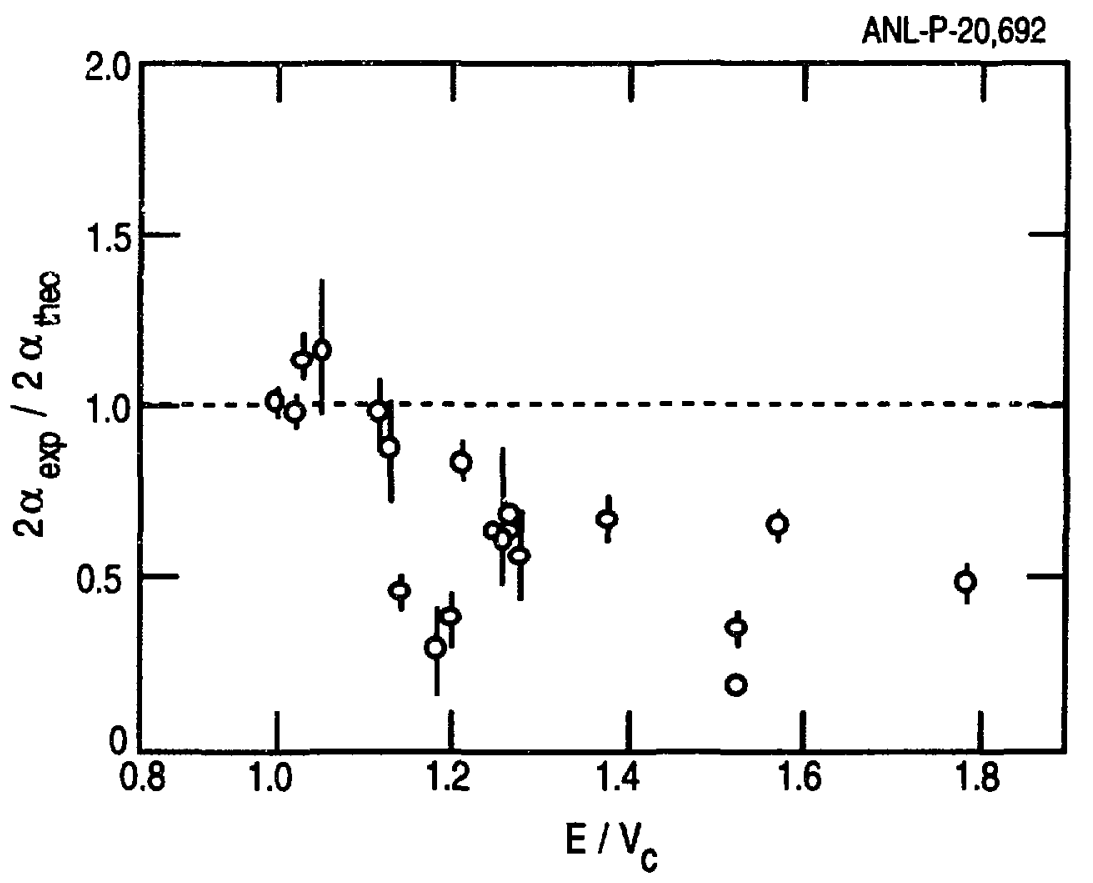

Fig. I-2. Ratio of the experimental slope parameters $2 \alpha_{\text {exp }}$ and the theoretical values (calculated from the respective binding energies) as function of the energy above the Coulomb barrier for two-neutron transfer reactions induced by projectiles ranging from ${ }^{36} \mathrm{~S}$ to ${ }^{64} \mathrm{Ni}$ on different target nuclei. 


\author{
a.5. Transfer Reactions in the Systems ${ }^{28} \mathrm{Si},{ }^{37} \mathrm{Cl}+{ }^{208} \mathrm{~Pb}$ at Energies Above \\ the Barrier (K. E. Rehm, S. Dixit,* M. Belbot,* W. K. Chung,* J. J. Kolata,* \\ K. Lamkin,* R. Tighe,* and M. Zahar*)
}

The energy dependence of the optical potential has been studied for the systems ${ }^{28} \mathrm{Si}$ and ${ }^{37} \mathrm{Cl}+$ ${ }^{208} \mathrm{~Pb}$ in the energy range from the Coulomb barrier $\mathrm{V}_{\mathrm{c}}$ to $\mathrm{E}=3 \cdot \mathrm{V}_{\mathrm{c}}$. Angular distributions for elastic scattering and inelastic excitations of low-lying states measured at these energies were analyzed within a coupled channels description. These measurements, which required sufficient resolution to separate the inelastic channels from the elastic scattering, were done at the split-pole spectrograph. In addition to the inelastic scattering, a variety of transîer reactions could be analyzed covering the region from quasi-elastic to deep-inelastic processes. These data allowed the study of the evolution of these processes as a function of the incident energy in great detail. A comparison with the predictions of various models is presently being performed.

*University of Notre Dame

\title{
a.6. Deep Inelastic Scattering Near the Coulomb Barrier (J. Gehring, K. Chan, M. Freer, D. Henderson, C. Jiang, K. E. Rehm, J. P. Schiffer, M. Wolanski, and A. Wuosmaa)
}

Traditional models of deep inelastic scattering invoke frictional forces to explain the dissipation of energy and are designed to predict the features of the process at high energies where the nuclear overlap and relative velocities of the colliding nuclei are large. They cannot be applied at energies below the Coulomb barrier where friction is negligible. The observation of a significant cross section for deep inelastic scattering at energies below the barrier requires a qualitatively different explanation.

In a continuation of previous work on $\mathrm{Ni}+\mathrm{Sn}$, we completed measurements of cross sections and angular distributions for deep inelastic scattering at two energies near the barrier in the system ${ }^{136} \mathrm{Xe}+{ }^{64} \mathrm{Ni}$. The cross sections are comparable to those measured earlier by Wolfs et al. in the system ${ }^{58} \mathrm{Ni}+{ }^{124} \mathrm{Sn}$. We obtained better statistics, better mass and energy resclution, and more complete angular coverage in the current experiment. As the measurements of this phenomenon were made on systems of closed-shell nuclei ( $\mathrm{Ni}, \mathrm{Sn}$ and $\left.{ }^{136} \mathrm{Xe}\right)$, we plan to make further measurements on the system ${ }^{124} \mathrm{Xe}+58 \mathrm{Ni}$ where the heavy partner does not have a closed shell. This will allow us to search for any differences in the cross sections or mass distributions due to the closed shell. It will also allow the observation of any differences between the neutron-poor ${ }^{124} \mathrm{Xe}+{ }^{58} \mathrm{Ni}$ and the neutron-rich ${ }^{136} \mathrm{Xe}+{ }^{64} \mathrm{Ni}$ systems.

a.7. Charge-State Dependence of Internal Conversion in ${ }^{83} \mathbf{K r}$ (E. R $\mathrm{hm}$, I. Ahmad, J. Gehring, B. G. Glagola, W. Kutschera, A. R. Barnett,* J. Copnell,* and W. R. Phillips*)

Analysis is almost complete of data obtained with the split-pole spectrometer on charge-changing events in a secondary ${ }^{83} \mathrm{Kr}$ beam containing a small fraction of nuclei in the $9.4-\mathrm{keV}$ isomeric state. These data provide information on how the $\mathrm{L}$-shell internal conversion coefficient of the 9.4-keV transition depends on ionic charge-state and elsctron configuration.

Two experiments were performed, both using Coulomb excitation of a ${ }^{83} \mathrm{Kr}$ beam in a Au target to provide the secondary beam. The first experiment used a bombarding energy of $650 \mathrm{MeV}$ and the second $735 \mathrm{MeV} .{ }^{83} \mathrm{Kr}$ ions were observed at $\theta_{\mathrm{L}}=25^{\circ}$ and $35^{\circ}$ in the first, and at $\theta_{\mathrm{L}}=22^{\circ}$ in the second experiment. A spectrum of $65-\mathrm{MeV}{ }^{83} \mathrm{Kr}$ ions scattered from a thin $\mathrm{Au}$ foil and detected

*University of Manchester, United Kingdom 
with a magnetic spectrograph at $25^{\circ}$ is shown in Fig. I-3. Monte Carlo simulations of the pattern of events observed in the focal plane of the spectrometer were used to extract the total decay probabilities $\lambda(q)$ for the $9.4-\mathrm{keV}$ level in ${ }^{83} \mathrm{Kr}$ ions of charge states $\mathrm{q}=28$ to 32 . Analysis of data from the first experiment gave uncertainties dominated by the low statistics obtained for charge-changing events. Analysis of the second experiment gave uncertainties determined by the presence of a low-energy tail $\left(\sim\right.$ few paits in $10^{4}$ ) in the beam incident on the Au target. Combination of the results from the experiment at $650 \mathrm{MeV}$ gives the following values for $\lambda(\mathrm{q}) \times$ $10^{6} \mathrm{sec}^{-1}$ for $\mathrm{q}=28$ to 32 successively: $3.99(28) ; 4.05(25) ; 3.86(22) ; 3.99(27)$ and $4.12(60)$. Calculations of internal conversion coefficients (ICC) were perfermed with wave functions from the MCDF code GRASP. Using a value for the $\gamma$-decay width $\lambda_{\gamma}=0.255(2) \times 10^{6} \mathrm{sec}^{-1}$, obtained from the measured lifetime in the neutral atom and the calculated ICC for the neutral atom of 16.545 , the following values for $\alpha_{L}(q)$ for $q=28$ to 32 are obtained: 14.7(12); 14.9(11); $14.1(9) ; 14.7(11)$ and $15.2(22)$. These results compare well with calculated values of $14.43(11)$; $14.24(11) ; 13.56(8) ; 13.68(8)$ and $14.06(12)$ for $q=28$ to 32 , respectively, where the errors are calculational and do not include the uncertainty due to errors in the M1/E2 mixing ratio for the 9.4$\mathrm{keV}$ transition. In addition, the calculations were performed for the lowest configurations of the charge states. Although there are strong arguments indicating that ions with $q=28$ to 31 are in their lowest electron configurations a few ns after exiting the Au target, for $q=32$ there exist longlived ${ }^{3} \mathrm{P}_{0,1,2}$ states from the configuration $(1 \mathrm{~s})^{2}(2 \mathrm{p})^{1}(2 \mathrm{p})^{1}$, and a fraction of ions in this configuration should be present in the $q=32$ beam when it enters the spectrometer. The $\alpha_{L}$ value for this fraction is lower than for the remaining fraction in the $(1 \mathrm{~s})^{2}(2 \mathrm{~s})^{2}: 1 \mathrm{~S}$ state because of lower $\mathrm{M} 1$ conversion on $\mathrm{p}$ electrons than on s electrons. If $50 \%$ of the $\mathrm{q}=32$ ions are in $3 \mathrm{P}$ states, the calculated $\alpha_{\mathrm{L}}(32)$ becomes 11.52(10). The 650-MeV data show no suggestion of a lowering of $\alpha_{L}(32)$. Although the absolute $\lambda$ (q) extracted from the 735-MeV experiment are less reliable than from the first data set, the relative values also suggest that $\alpha_{L}(32)$ is little different from the other coefficients. Hence the interesting possibility should be considered that a mechanism exists for quenching the ${ }^{3} \mathrm{P}$ levels in freely-moving Be-like $\mathrm{Kr}$ ions.

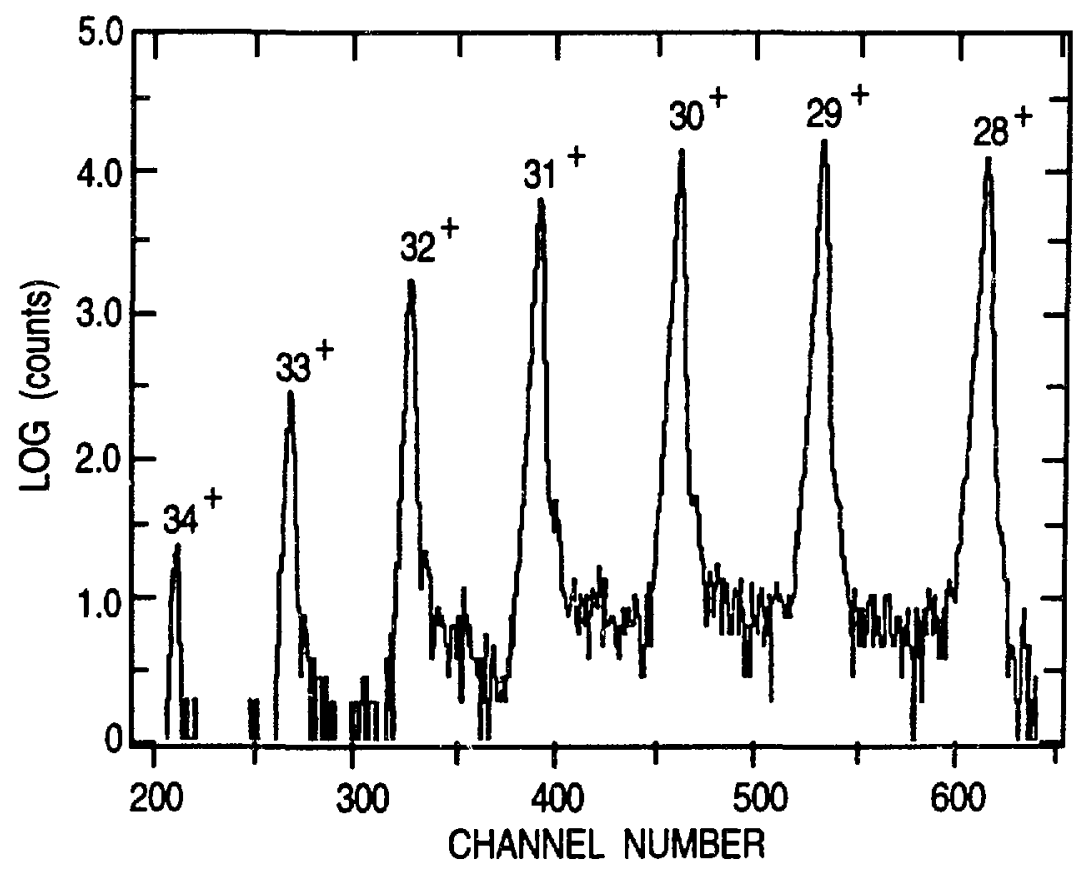

Fig. I-3. The pattern of events ("charge-state system") observed in the focal plane of the Enge magnetic spectrograph when $650-\mathrm{MeV}^{83} \mathrm{Kr}$ ions are scattered off a thin Au foil at $\theta=25^{\circ}$. 
a.8. Lifetimes of Isomeric States in ${ }^{83} \mathrm{Kr}$ and ${ }^{57} \mathrm{Fe}$ (I. Ahmad, E. Rehm, E. Kanter, W. Kutschera, and W. R. Phillips*)

In order to eliminate possible sources of uncertainty in the interpretation of experiments designed to measure changes with ionic charge state of internal conversion coefficients of the 9.4-keV isomer in ${ }^{83} \mathrm{Kr}$ and of the $14.4-\mathrm{keV}$ isomer in ${ }^{57} \mathrm{Fe}$, the lifetimes of these isomers were remeasured accurately.

A source of ${ }^{83} \mathrm{Rb}\left(\tau_{1 / 2}=86.2 \mathrm{~d}\right)$ was used to populate the $562-\mathrm{keV}$ level in ${ }^{83} \mathrm{Kr}$ which partially decays to the $94-\mathrm{keV}$ state. The time distribution of coincidences between the 553-keV $\gamma$ rays and 9.4-keV $\gamma$ rays deexciting the isomer was determined by observing the 553-keV $\gamma$ ray in a $\mathrm{Ge}$ detector and the coincident 9.4-keV $\gamma$ ray in a LEPS detector. A fast-slow coincidence system, in which the slow side placed gates on appropriate peaks in the detector, was used with a calibrated time-to-amplitude converter to measure the decay curve. The halflife of the 9.4-keV state was determined to be $t_{1 / 2}=155(1)$ ns. The most accurate value previously reported is $147(4)$ ns and a decay curve is shown in Fig. I-4.

A source of ${ }^{57} \mathrm{Co}\left(\tau_{1 / 2}=271 \mathrm{~d}\right)$ was used to populate the $136-\mathrm{keV}$ level in ${ }^{57} \mathrm{Fe}$ which partially decays to the 14.4-keV state. The time distribution of coincidences between the $121-\mathrm{keV} \gamma$ ray and the 14.4-keV $\gamma$ ray deexciting the isomer was measured using the same experimental arrangement as used for the ${ }^{83} \mathrm{Kr}$ experiment. The halflife of the $14.4-\mathrm{keV}$ state was determined to be $99.2(4)$ ns. The value quoted in Nuclear Data Sheets is $98.1(3) \mathrm{ns}$, which is an unweighted average of several experiments, the most recent performed in 1968.

*University of Manchester, United Kingdom

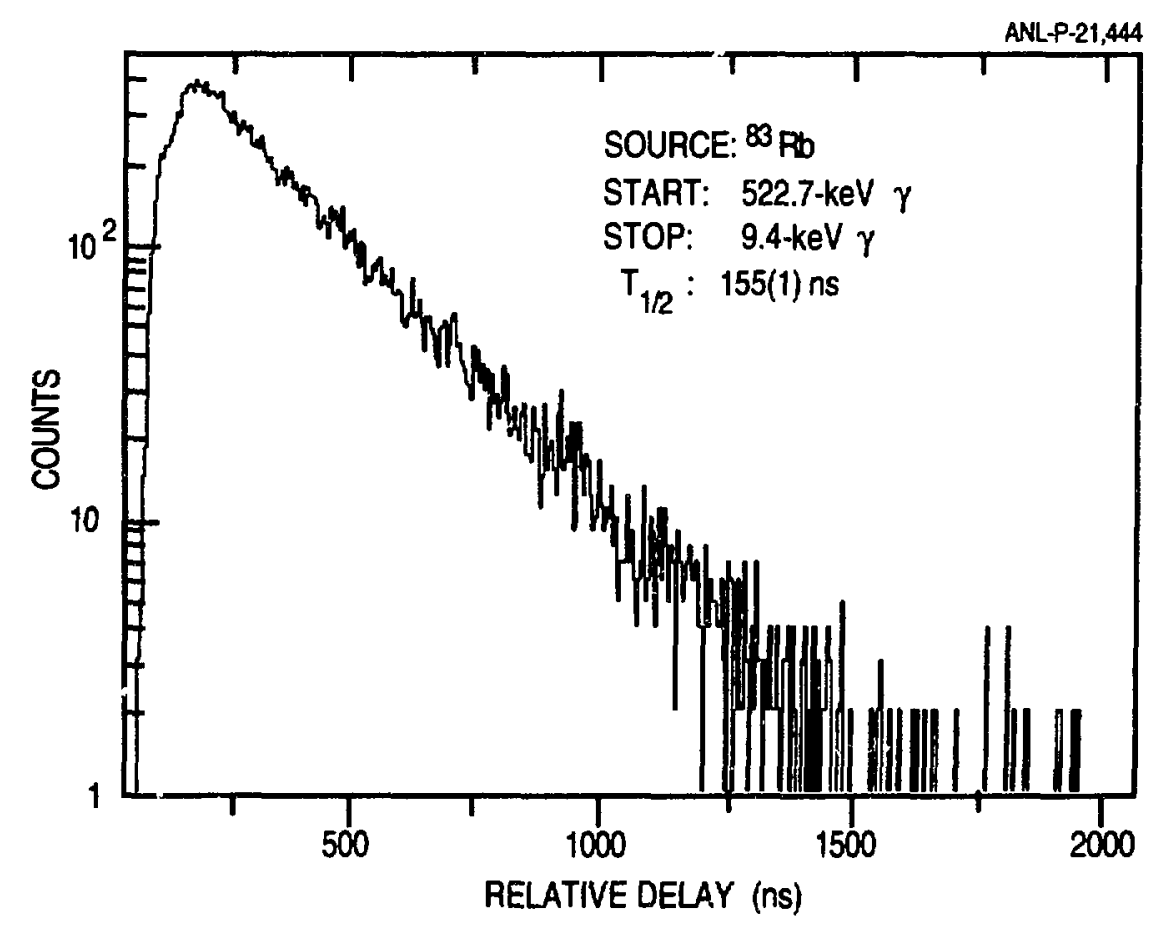

Fig. 1-4. Lifetime measurement of the $9.5^{i}-\mathrm{keV}$ state in ${ }^{83} \mathrm{Kr}$. 


\section{b. Search for Exotic Cluster States in Light Nuclei}

Calculations of the potential energy surfaces of light nuclei have suggested that extremely deformed configurations corresponding to linear chains of alpha particles might be sufficiently stable to be observable as resonances in the appropriate reaction channels. The prototypical examples of this type of state are the ${ }^{8} \mathrm{Be}$ ground state and the excited $0^{+}$state in ${ }^{12} \mathrm{C}$ at $7.6 \mathrm{MeV}$. From a completely different starting point, alpha-cluster-model calculations also lead to the same expectations.

We searched for such states using a high-resolution, highly-segmented detector array consisting of double-sided silicon strip detectors and associated electronics develofed at Argonne. Evidence for a six-alpha chain was observed in the excitation function for the ${ }^{12} \mathrm{C}\left({ }^{12} \mathrm{C},{ }^{12} \mathrm{C}\left(\mathrm{O}^{+} 2\right)\right)^{12} \mathrm{C}\left(\mathrm{O}^{+} 2\right)$ reaction which shows a very broad resonance that appears to correspond to a number of overlapping resonances with a rather particular relative phase. Theoretical work, inspired by this resuit, led to the speculation that such a degenerate band of overlapping states, based on a particular shell-model configuration, corresponds to a new type of quantum state - a "deformation eigenstate." Further experiments to verify these suppositions are in progress.

\section{b.1. Study of the ${ }^{12} \mathrm{C}+{ }^{12} \mathrm{C} \rightarrow{ }^{8} \mathrm{Be}$.s.s. $+160(4 \alpha)$ Reaction}

(A. H. Wuosmaa, R. R. Betts, and M. Freer)

Previous studies of the inelastic scattering reaction ${ }^{12} \mathrm{C}+{ }^{12} \mathrm{C} \rightarrow{ }^{12} \mathrm{C}\left(\mathrm{O}^{+} 2\right)+{ }^{12} \mathrm{C}\left(\mathrm{O}^{+} 2\right)$ identified a strong resonance-like feature in the excitation function for this channel, peaked at a center-ofmass energy of $E_{\mathrm{cm}}=32.5 \mathrm{MeV}$. The excitation energy in the composite system ${ }^{24} \mathrm{Mg}\left(\mathrm{E}_{\mathrm{x}}=46.4\right.$ $\mathrm{MeV}$ ), as well as the dominant angular momenta (14-16 $\hbar)$, suggest that this structure couid be related to the population of alpha-particle chain configurations in ${ }^{24} \mathrm{Mg}$, similar to those predicied by Nilsson-Strutinsky and cranked alpha-cluster model calculations. If this interpretation is correct, the decay branch observed in the ${ }^{12} \mathrm{C}+{ }^{12} \mathrm{C}$ inelastic scattering channel would correspond to the symmetric decay mode of this chain structure. It is likely, however, that different decay modes also exist for such a configuration. One such decay could be by the emission of a ${ }^{8} \mathrm{Be}$ nucleus, leaving ${ }^{16} \mathrm{O}$ in a highly excited state corresponding to the 4- $\alpha$ particle chain structure in that nucleus. These extended configurations in ${ }^{16} \mathrm{O}$ have been associated with resonances observed in the $\alpha+{ }^{12} \mathrm{C} \rightarrow{ }^{8} \mathrm{Be}+{ }^{8} \mathrm{Be}$ and $\alpha+{ }^{12} \mathrm{C} \rightarrow \alpha+{ }^{12} \mathrm{C}\left(0^{+},\right)$reactions. As the chain structures in ${ }^{16} \mathrm{O}$ decay to either 2 additional ${ }^{8} \mathrm{Be}$ nuclei or to $\alpha+{ }^{12} \mathrm{C}\left(\mathrm{O}^{+} 2\right)$, the resulting final state is 6 alpha particles.

We performed a measurement to study in detail this and other reaction pathways that lead from ${ }^{12} \mathrm{C}$ $+{ }^{12} \mathrm{C}$ to 6 alpha particles. The experiment was carried out using an array of four double-sided silicon strip detectors, placed at angles of 15 and 35 degrees on either side of the beam, at distances of 14 and $17 \mathrm{~cm}$. The resulting solid angle covered by this array is approximately 430 msr, effectively divided into 1024 pixel regions. A ${ }^{12} \mathrm{C}$ beam from ATLAS bombarded $50-\mu \mathrm{g} / \mathrm{cm}^{2}$ targets at 5 energies in the region of the structure in the ${ }^{12} \mathrm{C}+{ }^{12} \mathrm{C}$ excitation function. All events with a measured particle fold of $\mathbf{N}=3$ or greater were recorded. From this data set, approximately 1,000 6-fold events and 10,000 5-fold events were obtained at each energ $\%$. Under the assumption that for any event with more than four fragments in the final state, all paiticles are alpha particles, momentum reconstruction permits a complete characterization of the 6-body final state. 


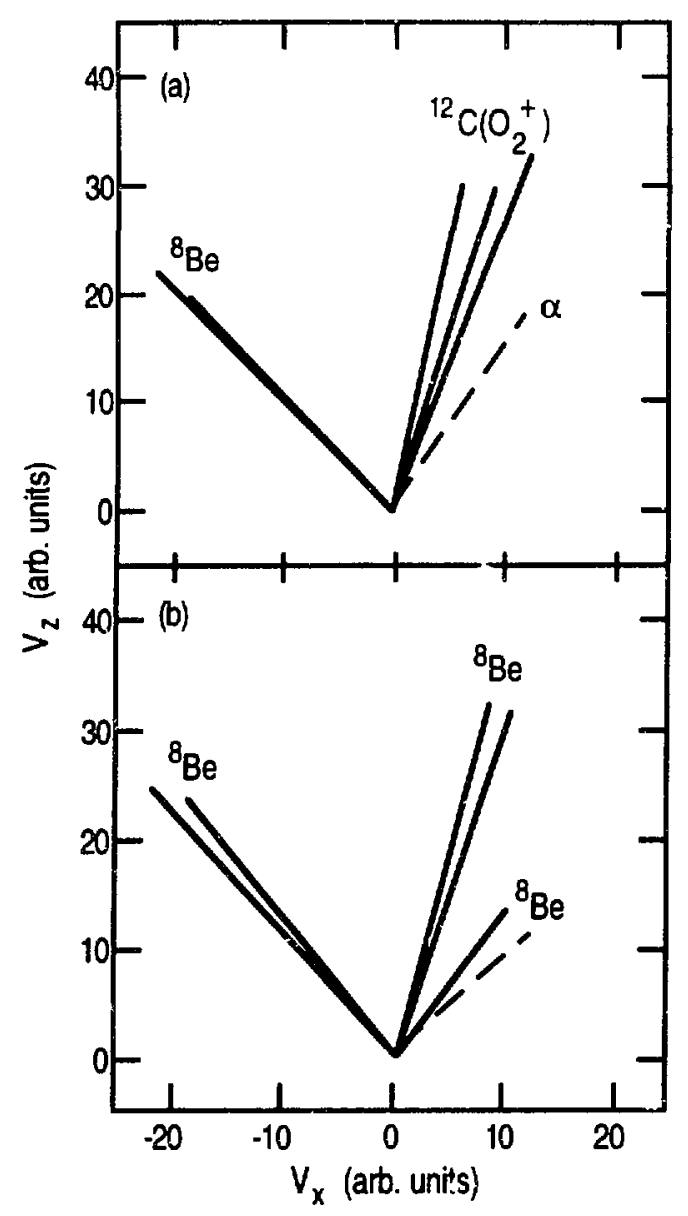

Fig. 1-5. Diagram showing the alpha-particle velocities for events identified as:

(a) ${ }^{12} \mathrm{C}+{ }^{12} \mathrm{C} \rightarrow{ }^{8} \mathrm{Be}+{ }^{16} \mathrm{O}^{*}\left(\alpha+{ }^{12} \mathrm{C}\left(\mathrm{O}^{+}{ }_{2}\right)\right)$ and $(b){ }^{12} \mathrm{C}+{ }^{12} \mathrm{C} \rightarrow{ }^{8} \mathrm{Be}+{ }^{16} \mathrm{O}^{*}\left({ }^{8} \mathrm{Be}+{ }^{8} \mathrm{Be}\right)$.

The dashed lines are reconstructed velocities for unobserved alpha particles.

From these data, we conclusively identified the reaction ${ }^{12} \mathrm{C}\left({ }^{12} \mathrm{C},{ }^{8} \mathrm{~B} \text {.s. }\right)^{16} \mathrm{O}^{*}(4 \alpha)$, where the recoiling 160 decays into either two ${ }^{8} \mathrm{Be}$ nuclei, or to $\alpha+{ }^{12} \mathrm{C}\left(0^{+} 2\right)$. Figure I-5 shows diagrams of the measured (solid lines) and reconstructed (dashed lines) alpha particle velocities for events corresponding to these two 160 decay sequences. The excitation energy of the recoiling $16 \mathrm{O}$ is determined from either the scattering angle and kinetic energy of the primary ${ }^{8} \mathrm{Be}$ nucleus, or from the kinematic correlations of the 160 decay products. Figure I-6 shows ${ }^{16} \mathrm{O}$ excitation-energy spectra for events in which the 160 decays into (a) any combination of 4 alpha particles, (b) to $\alpha+$ ${ }^{12} \mathrm{C}\left(\mathrm{O}^{+} 2\right)$, and (c) to ${ }^{8} \mathrm{Be}+{ }^{8} \mathrm{Be}$. We observed discrete levels in ${ }^{16} \mathrm{O}$ populated at excitation energies near 17 and $19 \mathrm{MeV}$, in the same range of excitation energies where strong resonance behavior in $\alpha+{ }^{12} \mathrm{C}$ scattering has been interpreted as reflecting the population of elongated alphaparticle configurations in 160 . The yield for the two- ${ }^{8} \mathrm{Be}$ decay of 160 is considerably smaller than that of the $\mathrm{O}^{t}+{ }^{12} \mathrm{C}\left(\mathrm{O}^{+} 2\right)$ decay, as expected from barrier-penetration considerations. The identification of these !evels and their association with resonances in $\alpha+{ }^{12} \mathrm{C}$ scattering could provide strong additional evidence in favor of the picture that elongated alpha-particle cluster configurations in ${ }^{24} \mathrm{Mg}$ may play an important role in these scattering reactions. Further experiments are planned to examine in detail the properties of the levels populated in ${ }^{16} \mathrm{O}$ through these reactions. 


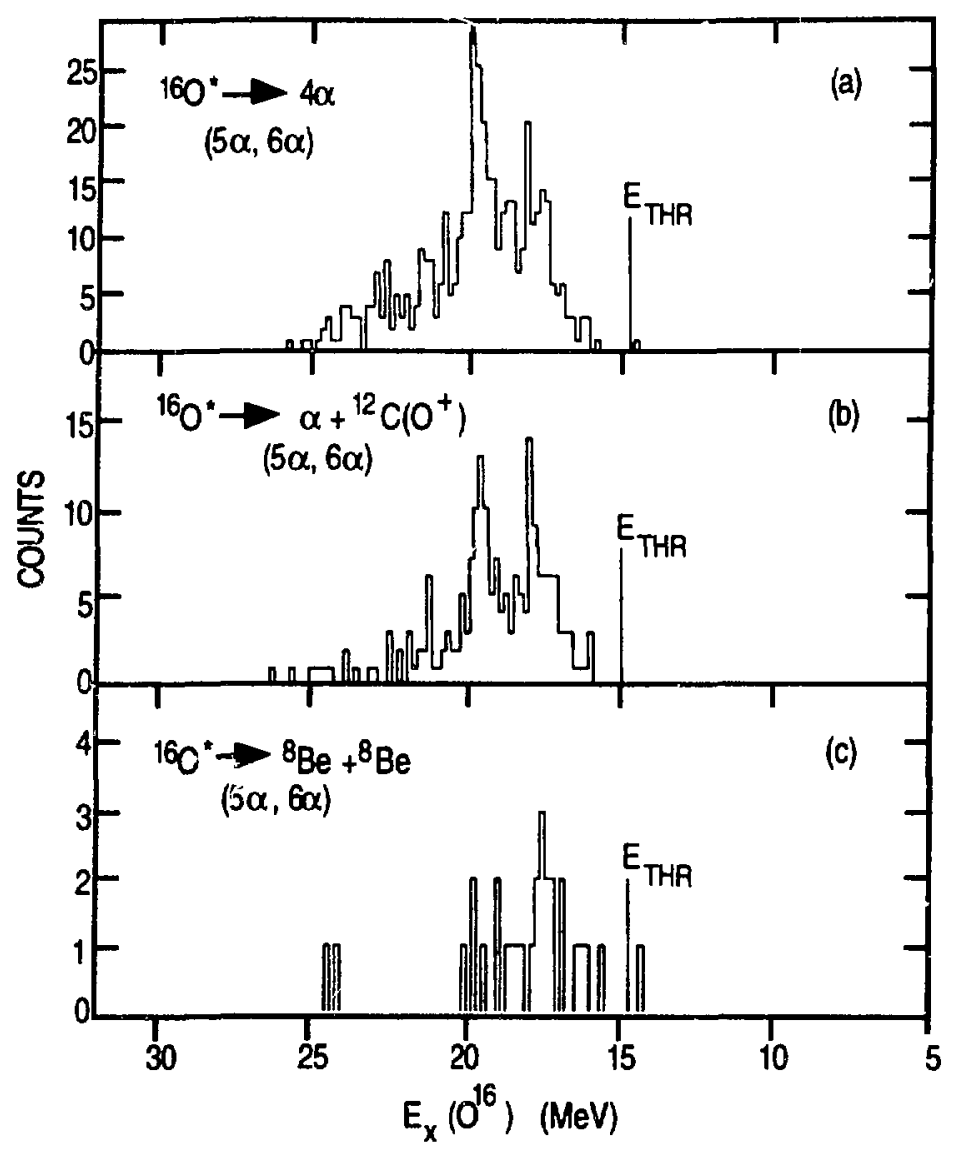

Fig. I-6. ${ }^{16} 0$ excitation-energy specira derived from 5 and 6 alpha coincidence events where the ${ }^{16} O$ decays to (a) any combination of 4 alpha particles, (b) $\alpha+{ }^{12} C\left(0^{+}{ }_{2}\right)$, and $(c)^{8} B e+{ }^{8} B e$.

\section{b.2. ${ }^{20} \mathrm{Ne}+{ }^{20} \mathrm{Ne}$ Elastic and Inelastic Scattering (S. Barrow,* Y. Miao,*}

J. T. Murgatroyd,* K. Pohl,* N. Wimer* R. W. Zurmühle* A. H. Wuosmaa, M. Freer, and R. R. Betts)

Resonance behavior in elastic and inelastic scattering of light, near-symmetric nuclei, is a welldocumiented phenomenon. Although the origin of these resonances is not completely understood, it is believed that they are associated with the formation of deformed configurations in the intermediate compound system. These resonance structures are particularly pronounced in target projectile systems composed of $\mathrm{A}=4 \mathrm{n}$ nuclei. However, due to experimental difficulties in the study of the ${ }^{20} \mathrm{Ne}+{ }^{20} \mathrm{Ne}$ reaction, the experimental data has remained incomplete for this system.

The development of the ECR source at ATLAS has facilitated the production of noble element beams. In conjunction with a ${ }^{20} \mathrm{Ne}$ target gas cell constructed at the University of Pennsylvania, ${ }^{1}$ the first ${ }^{20} \mathrm{Ne}$ beam from ATLAS was employed to perform an excitation function, spanning beam energies from 70 to $83 \mathrm{MeV}$ in $250-\mathrm{keV}$ steps. Using the kinematic coincidence-detection technique, the masses of the reaction products and the associated $\mathrm{Q}$ value were determined simultaneously. This allowed the resonance behavior of the elastic, several inelastic channels and alpha transfer channels to be studied separately.

*University of́ Pennsylvania, ${ }^{1}$ S. P. Barrow, Ph.D. Thesis, University of Pennsylvania (1994) 
The results of these measurements were somewhat surprising. Figure I-7(a) shows excitationfunction data for low-lying excitations in the $20 \mathrm{Ne}+{ }^{20} \mathrm{Ne}$ final state. Very little narrow structure is observed in the excitation function for these channels, although some peaks are observed for final states at higher excitation energy. Elastic-scattering angular distribution measurements reveal that the dominant angular momenta are consistent with the grazing angular momentum, in contrast to the results for the ${ }^{24} \mathrm{Mg}+{ }^{24} \mathrm{Mg}$ case, where the dominant angular momenta are 4 to 6 units higher than grazing. More interesting behavior is seen in the $\alpha$-transfer channel leading to the 160 $+{ }^{24} \mathrm{Mg}$ final state [Fig. 1-7(b)], where prominent $2 x$ xitation-function structures with widths on the order of $\Delta \mathrm{E}_{\mathrm{c} . \mathrm{m} .}=400-500 \mathrm{keV}$ are present. These results suggest that if states in ${ }^{40} \mathrm{Ca}$ with quasi-molecular properties exist, their overlap with the ${ }^{20} \mathrm{Ne}+20 \mathrm{Ne}$ charnel is small, and their structure more likely resembles a somewhat less deformed configuration than those suggested to be responsible for resonance behavior in the ${ }^{24} \mathrm{Mg}+{ }^{24} \mathrm{Mg}$ system.

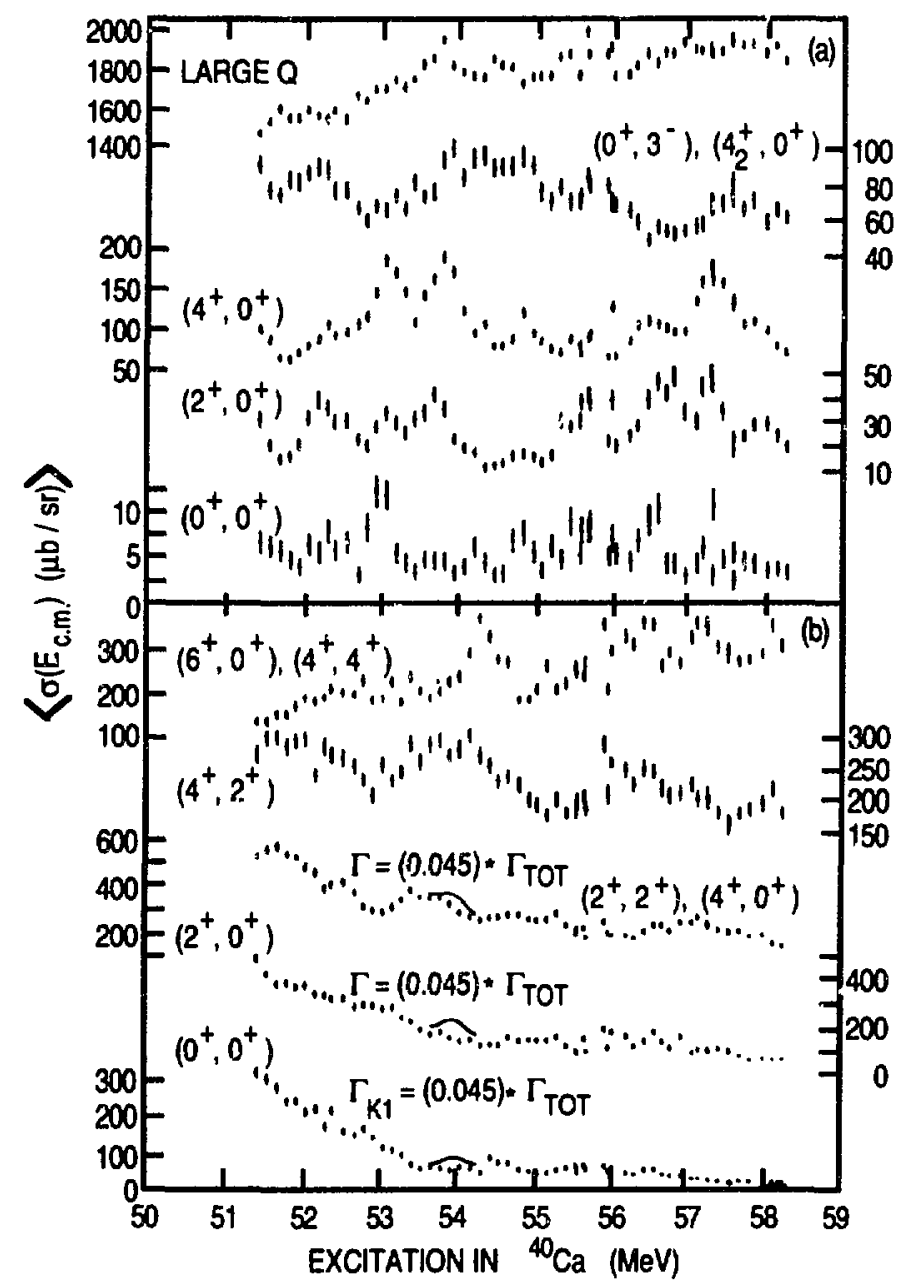

Fig. I-7. Excitation functions for (a) ${ }^{20} \mathrm{Ne}+{ }^{20} \mathrm{Ne}$ inelastic scatterirg and $(b)$ the ${ }^{20} \mathrm{Ne}\left({ }^{20} \mathrm{Ne},{ }^{16} \mathrm{O}\right)^{24} \mathrm{Mg}$ reaction. 


\section{b.3. Search for Many-Body Decay of Resonances in the ${ }^{24} \mathbf{M g}+{ }^{24} \mathrm{Mg}$ System \\ (A. H. Wuosmaa, M. Freer, R. W. Zurmühle,* C. Lee,* S. P. Barrow,* Y. Miao,* \\ N. Wimer,* B. R. Fulton, $\dagger$ and A. Murphy†)}

Prominent resonance behavior has long been known to occur in systems involving nuclei in the s-d shell composed of integral numbers of alpha particles. One of the most striking examples of this behavior is found in the ${ }^{24} \mathrm{Mg}+{ }^{24} \mathrm{Mg}$ system, where excitation functions reveal 3 groups of strong resonances at excitation energies between 60 and $70 \mathrm{MeV}$, with spins from 36 to $40 \hbar$ in the composite system ${ }^{48} \mathrm{Cr}$. These resonances were interpreted as arising from the population and subsequent binary decay of highly elongated configurations in the composite system ${ }^{48} \mathrm{Cr}$. One puzzling feature of these resonances, however, is that only a small fraction, approximately $30 \%$, of the total resonance strength is observed in the elastic and inelastic scattering channels for these peaks. The resonances were observed in alpha transfer reactions leading to the ${ }^{20} \mathrm{Ne}+{ }^{28} \mathrm{Si}$ final state, hut the resonance cross sections for these transfer reactions are far too small to explain the missing strength. One possibility is that these resonances possess large decay widths to particle unbourd final states, e.g. $\alpha+{ }^{20} \mathrm{Ne}+{ }^{24} \mathrm{Mg}, 2 \alpha+{ }^{20} \mathrm{Ne}+20 \mathrm{Ne}$, etc. These final states are, in general, quite difficult to study with a conventional detector setup.

We used an experimental setup consisting of two highly-segmented, large-area double-sided silicon strip detectors (DSSD) combined with a large solid-angle gas ionization telescope to study many-body fillal states in the energy region of one strong resonance observed in the ${ }^{24} \mathrm{Mg}+{ }^{24} \mathrm{Mg}$ system. A ${ }^{24} \mathrm{Mg}$ beam from the University of Pennsylvania Tandem Accelerator was used to bombard targets consisting of $30 \mu \mathrm{g} / \mathrm{cm}^{2}$ of ${ }^{24} \mathrm{Mg}$ evaporated on $15-\mu \mathrm{gg} / \mathrm{cm}^{2}{ }^{12} \mathrm{C}$ backings at two energies, $E_{\mathrm{cm}}=45.70$ and $46.25 \mathrm{MeV}$, at the peak and off the peak, respectively, of one strong ${ }^{24} \mathrm{Mg}+{ }^{24} \mathrm{Mg}$ elastic scattering resonance. Data were obtained for one week at each beam energy. Coincidences between any hit in either one or both of the DSSDs, and in the gas telescope were recorded in event mode. Particle identification was obtained from the relative time-of-flight information from the two DSSDs, as well as from the $\mathrm{E}-\Delta \mathrm{E}$ data in the gas telescope.

Several exit channels have been identified, including ${ }^{12} \mathrm{C}+{ }^{32} \mathrm{~S}+\alpha,{ }^{16} \mathrm{O}+{ }^{28} \mathrm{Si}+\alpha$, and ${ }^{20} \mathrm{Ne}+$ ${ }^{24} \mathrm{Mg}+\alpha$. A preliminary analysis indicates that the dominant reaction mechanism is inelastic scattering or $\alpha$ transfer to $\alpha$-unbound levels in either ${ }^{24} \mathrm{Mg}$ or ${ }^{28} \mathrm{Si}$, followed by $\alpha$-particle emission, although the ${ }^{12} \mathrm{C}+{ }^{32} \mathrm{~S}+\alpha$ data cannot be explained by such a mechanism. There is also some evidence for the enhancement of these yields on resonance, although the statistics are somewnat marginal. Finally, some weak evidence is seen for the population of fissioning states in ${ }^{44} \mathrm{Ti}$, via the ${ }^{24} \mathrm{Mg}\left({ }^{24} \mathrm{Mg}, \alpha\right){ }^{44} \mathrm{Ti}$ reaction, however these results require a more sensitive experiment to examine this reaction in detail. We are planning such an experiment to run at ATLAS, using a large array of double-sided silicon strip detectors, which should provide considerably more information about these many-body final states in the ${ }^{24} \mathrm{Mg}+{ }^{24} \mathrm{Mg}$ system.

*University of Pennsylvania, $\nmid$ Birmingham University, United Kingdom

\section{c. Fusion and Fission Reactions}

It is generally assumed that the sub-barrier fusion enhancement observed in reactions between heavy ions can be explained satisfactorily by including elastic and inelastic scattering together with the fusion channel in a coupled-channels description. Recently, various heavy systems have been investigated where deviations from a coupled-channels description were observed. To get a better understanding of possible causes for these discrepancies, fusion cross sections in the systems $58,64 \mathrm{Ni}+{ }^{92,},{ }^{00} \mathrm{Mo}$ and $58,64 \mathrm{Ni}+{ }^{78,86} \mathrm{Kr}$ were measured. The nuclei involved in the reactions differ by up to 14 units in neutron number, and in their internal structure span the range from closed-shell to transitional nuclei. The largest fusion enhancements were observed for transitional nuclei, especially ${ }^{78} \mathrm{Kr}$, pointing to a strong influence of the polarizability on the strength of the fusion process. This effect is not yet understood theoretically. 
The dynamics of fission and quasi-fission processes is a topic of great current interest. The theoretical models describing the re-arrangement of nuclear matier taking place in these processes are as yet rather simple and clo not converge in predicting crucial dynamical observables such as mass flow and time-scales. The description of fission-like processes in terms of diffusion has recently proved fruitful, but the input parameters in such a description, namely the dissipation and inertial tensors must be provided by other models. To advance in this area of study it is therefore of critical importance to provide experimental data which are sensitive to the dynamics in lowenergy nuclear reactions. The present program aims tc provide such comprehensive data.

A new program that emerged recently is the study of exotic nuclei far away from the valley of beta stability and extending to very heavy elements. This new program blossomed because of the availability of high-intensity heavy-ion beams at ATLAS, and the successful operation of the FMA. Exotic nuclei far from the valley of stability are usually produced with very small cross sections. The separation of background reactions is much improved with the recoil implantation system installed recently at the Fragment Mass Analyzer (FMA), where nuclei are identified according to their mass with good mass resolution (up to 525:1) in the focal plane and implanted in a Si-detector (segmented into 2304 pixels) located behind the focal plane detector. A subsequent alpha or proton-decay at the implantation site together with time-of-flight and recoil energy signals, reduced the background drastically so that cross sections in the nanobarn and sub-nanobarn level can be measured.

\section{c.1. Measurement of Fusion Cross Sections for ${ }^{58,64} \mathrm{Ni}+{ }^{92,100} \mathrm{Mo}$ Ising the Gas-Filled Magnet Technique (K. E. Rehm, J. Gehring, B. G. Glagola, D. J. Henderson, W. Kutschera, M. Paul,* F. Soramel, $†$ and A. H. Wuosmaa)}

The enhancement of the cross sections for heavy-ion-induced fusion reactions at sub-barrier energies was studied extensively during the last 10 years. In addition to the increased fusion probability, a strong dependence of the fusion yield on the neutron number in projectile and target was observed for various lighter systems, (e.g. $\mathrm{Ni}+\mathrm{Si}, \mathrm{Ni}+\mathrm{S}, \mathrm{Ni}+\mathrm{Ni}$ ). We studied the fusion cioss sections in the heavier systems $58,64 \mathrm{Ni}+92,100 \mathrm{Mo}$ using the gas-filled magnet technique.

The experiments were performed with $\mathrm{Ni}$ beams of $204-260-\mathrm{MeV}$ incident energy in the split-pole spectrograph, which was filled with $\mathrm{N}_{2}$ at a pressure of 0.3 torr. Because of charge-changing collisions between the ions and the residual gas molecules, the charge-state distributions or the evaporation residues (ERs) and the projectiles are centered around an average charge state $\bar{q}$, with a width that is only a few $\mathrm{cm}$ wide as measured in the focal plane of the spectrograph. The difference in the magnetic rigidity and the time of flight between the ERs and the incident ions allows for a clean separation of the fusion products from the elastically scattered beam particles even at very small scattering angles.

The data from these experiments are completely analyzed. A comparison with the results from a coupled-channels calculation including one- and two-phonon excitations in projectile and target, gives good agreement for ${ }^{53,64} \mathrm{Ni}+{ }^{92} \mathrm{Mo}$ and ${ }^{64} \mathrm{Ni}+{ }^{100} \mathrm{Mo}$, while for the ${ }^{58} \mathrm{Ni}+{ }^{100} \mathrm{Mo}$ system an additional enhancement of the fusion yield at low energies is observed that cannot be explained by the inclusion of multi-phonon coupling effects (Fig. I-8). We are presentiy studying the possible influence of transfer reactions on this discrepancy.

*Hebrew University, Ierusalem, Israel, †University of Padova, Italy 


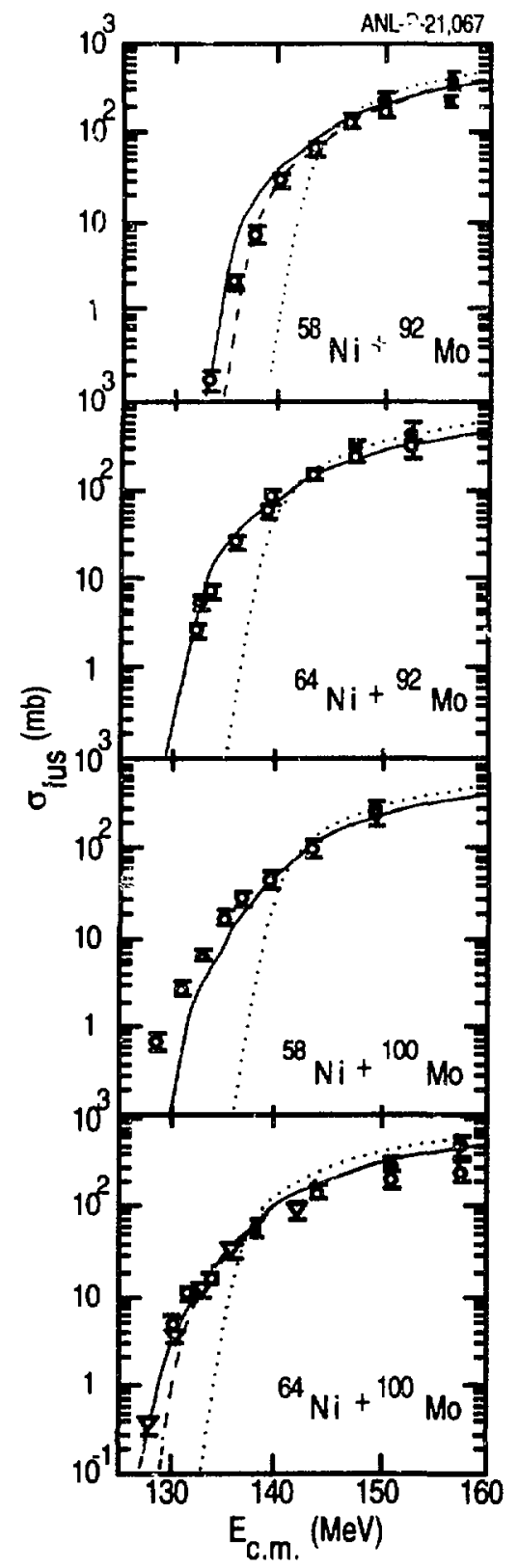

Fig. I-8. Cross sections for the production of evaporation residues in the systems $58 \mathrm{Ni}+{ }^{92,100} \mathrm{Mo}$ as function of the bombarding energy. The solid points include contributions from fission processes. The dotted lines are the result of a one-dimensional barrier penetration calculation. The dashed and solid curves are calculated within the coupled-channels formalism including one-phonon and one-plus-two-phonon couplings, respectively.

\section{c.2. Measurements of Fusion Cross Sections in the Systems ${ }^{58,64} \mathrm{Ni}+{ }^{78,86} \mathrm{~K}$ r} (K. E. Rehm, J. L. Chiang, H. Esbunsen, B. Crowell, J. Gehring, b. Glagola, M. Rhein, and A. H. Wuosmaa)

In continuation of our investigations of the nuclear structure dependence of the sub-barrier fusion enhancement in heavy-ion induced reactions, we have studied the systems ${ }^{58,64} \mathrm{Ni}+{ }^{78,86} \mathrm{Kr}$ at energies in the vicinity of the Coulomb barrier. Krypton ions were selected because, similar to the Mo case, there are strong changes in the nuclear structure when the neutron number is changed. However, different from Mo, where the softness of the nucleus changes with increasing neutron number, the most collective nucleus for the $\mathrm{Kr}$ case is the neutron-deficient ${ }^{78} \mathrm{Kr}$.

The experiment was performed with $\mathrm{Kr}$ beams from the positive-ion injector using enriched $78,86 \mathrm{Kr}$ gas in the ECR ion source. The separation of evaporation residues from the elastically scattered particles was achieved by using their difference in time-of-flight and the magnetic rigidity in a gas-filled magnet. 
The ${ }^{78} \mathrm{Kr}$-induced experiments are completely analyzed. For the ${ }^{86} \mathrm{Kr}+{ }^{58,64} \mathrm{Ni}$ case, an additional run is scheduled for 1994. A preliminary comparison of the ${ }^{78} \mathrm{Kr}+{ }^{58,64} \mathrm{Ni}$ data with the results of coupled-channels calculations including one- and two-phonon excitations reveals enhanced fusion yields at the lowest bombarding energies indicating a possible influence of the softness or polarizability of a nucleus on the fusion enhancement at low energies.

\section{c.3. High Energy $\gamma$-Rays from ${ }^{252}$ Cf Spontaneous Fission (B. B. Back, D. J. Hofman, * C. P. Montoya, * S. Schadmand,* R. Varma,* and P. Paul*)}

The spontaneous fission decay of ${ }^{252} \mathrm{Cf}$ has been analyzed in a statistical model with emphasis on describing high energy $\gamma$-ray spectra reported recently. An enhanced $\gamma$ emission in the range from 3 to $10 \mathrm{MeV}$ which is observed for nearly symmetric mass splits is readily understood as a result of the different fragment excitation energies. The model includes a viscous motion to the scission point with the possibility of pre-scission $\gamma$ emission. It was found that even with saddle-toscission times of $\tau_{\mathrm{sc}}<66 \times 10^{-19} \mathrm{~s}$, the maximum consistent with pre-scission neutron multiplicities, prescission $\gamma$ rays are overwhelmed by $\gamma$ emission from the final fragments. Thus, the strong angular anisotropy of $\gamma$-rays in the range $\mathrm{E}_{\gamma}=8-12 \mathrm{MeV}$ reported recently is unexplained within the present understanding of the fission process (see Fig. I-9).

*SUNY at Stony Brook

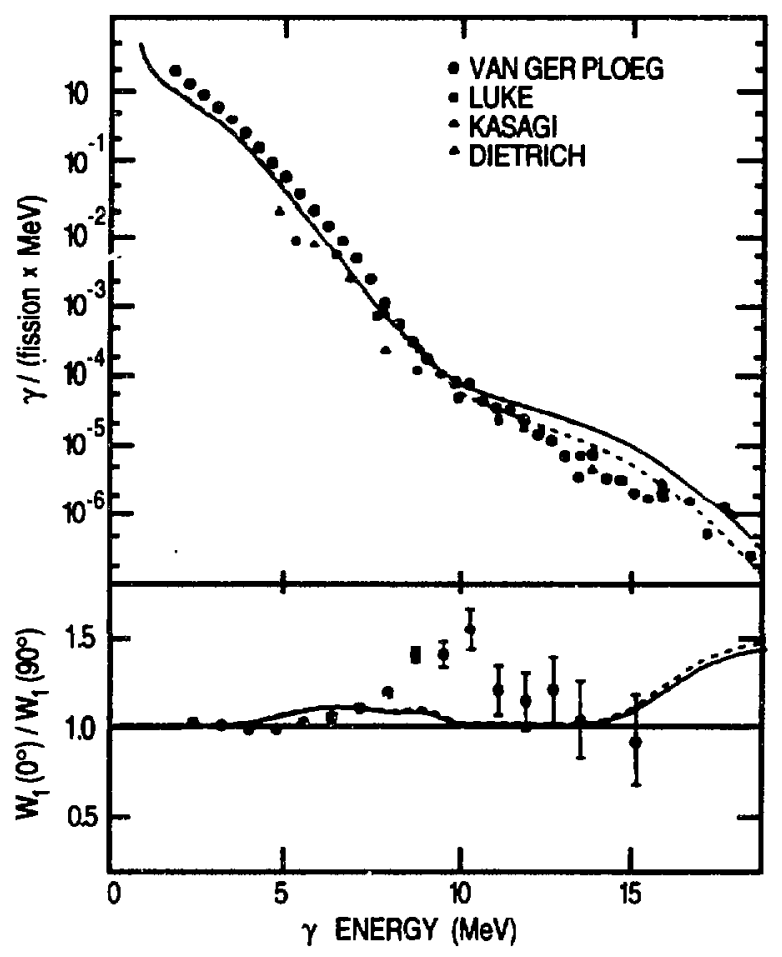

Fig. 1-9. Top panel: Comparison of the calcuiated $\gamma$ spectra (solid curve) to experimental data (filled circles [1], solid squares [2], solid triangles [3], and open triangles [4]). Bottom panel: The calculated anisotropy (solid curve) arising from Doppler shifts is compared to experimental data [1] (filled circles). The dashed curve represents a fit assuming a reduced initial excitation energy (see text).

${ }^{1}$ H. van der Ploeg et al., Phys. Rev. Lett. 68,3145 (1992), ${ }^{2}$ S. J. Luke et al., Phys. Rev. C 44 , 1548 (1991), 3J. Kasagi et al., Nucl. Phys. Soc. Jpn. 58, 620 (1989), 4F. S. Dietrich et al., Phys. Rev. C 10, 795 (1974). 


\section{c.4. Time-Scale in Quasi-Fission Reactions (B. B. Back, P. Paul,* J. Nestler,* K. S. Drese,* D. J. Hofman,* S. Schadmand,* and R. Varma*)}

The quasi-fission process arises from the hindrance of the complete fusion process when heavyion beams are used: The strong dissipation in the system tends to prevent fusion and leads the system towards reseparation into two final products of similar mass, reminiscent of a fission process. This dissipation slows down the mass transfer and shape transformation and allows for the emission of high energy $\gamma$ rays during the process, albeit with a low probability. Giant dipole $\gamma$ rays emitted during this time have a characteristic spectral shape and may thus be discerned in the presence of a background of $\gamma$ rays emitted from the final fission-like fragments. Since the rate of GDR $\gamma$ emission is very well established, the strength of this component may be used to measure the time scale of the quasi-fission process. In this experiment we have studied the reaction between $368-\mathrm{MeV} 58 \mathrm{Ni}$ and a ${ }^{165} \mathrm{Ho}$ target, where deep inelastic scattering and quasifission processes are dominant. Coincidences between fission fragments (detected in four positionsensitive avalanche detectors) and high-energy $\gamma$ rays (measured in a 10" $\times 10$ " actively shielded $\mathrm{NaI}$ detector) were registered. Beams were provided by the Stony Brook Superconducting Linac.

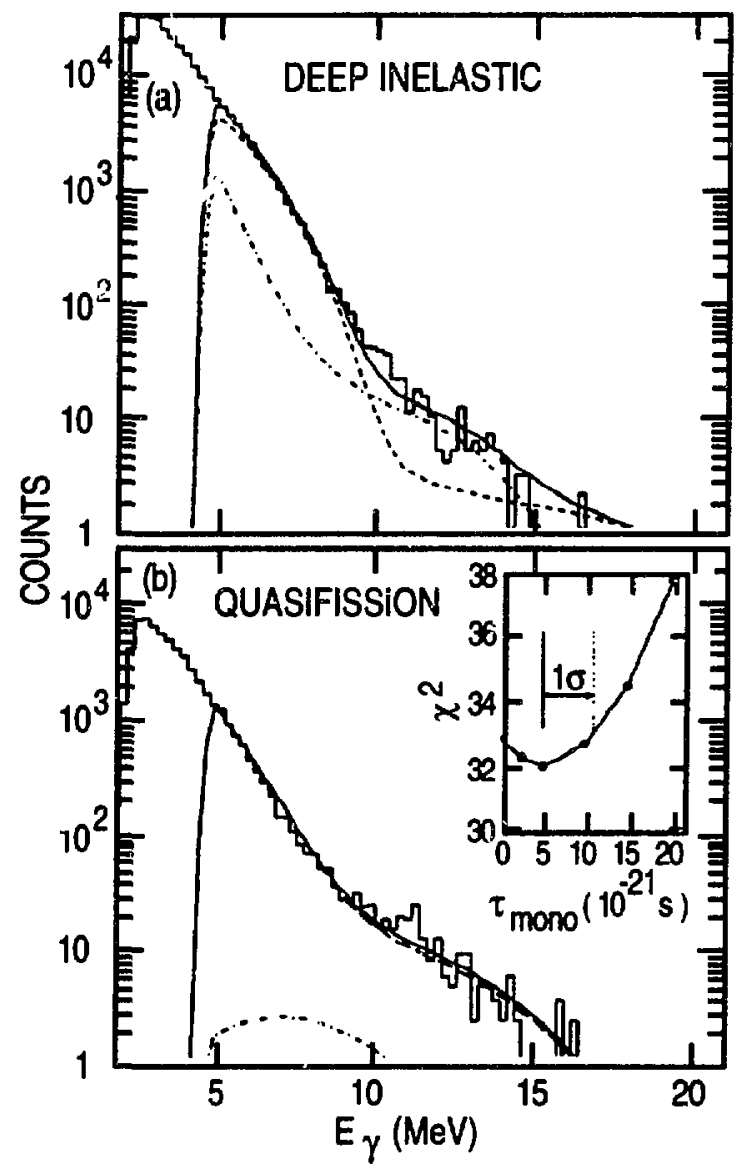

Fig. I-10. (a) Top: experimental $\gamma$ ray energy spectrum (histogram) observed in coincidence with deep elastic scattering events. The curves are fits assuming statistical emission of GDR $\gamma$ rays from the PLF (dashed) and TLF (dot-dashed) and their sum (solid curve) assuming almost equal energy sharing. (b) Bottom: experimental $\gamma$ ray spectrum (histogram) observed in coincidence with fission events. The curves are fits assuming statistical emission from the fission fragments only (dashed line) and the sum (solid line) including remission from the intermediate system (dot-dashed) calculated for $\tau_{\text {mono }}=510^{-21} \mathrm{~s}$. The $\chi^{2}$ dependence on $\tau_{\text {mono }}$ is illustrated in the insert. 
The $\gamma$-ray spectrum associated with deap inelastic scattering events is well reproduced by statistical cooling of projectile and target-like fragments with close to equal initial excitation energy sharing. The $\gamma$ spectrum associated with quasifission events is described well by statistical emission from the fission fraginents alone, with only weak evidence for GDR emission from the mono-nucleus (see Fig. I-10). A $1 \sigma$ limit of $\tau_{\mathrm{ss}}<11 \times 10^{-21} \mathrm{~s}$ is obtained for the mono-nucleus lifetime, which is consistent with the lifetime obtained from quasifission fragment angular distributions.

\section{c.5. Time Scale of the Fission Process (B. B. Back, P. Paul,* C. P. Montoya,* D. J. Hofman,* S. Schadmand,* and R. Varma*)}

It was realized recently that the fission process at high excitation energies is not wel! described by the standard statistical model. It was observed that the fission rate is smaller than this model precicts by using the neutron emission rate as a comparison standard. This reduction of the fission rate is a natural consequence of a description of fission as a diffusion process which is associated with a dissipation coefficient for the fission motion. The magnitude and temperature of the energy dissipation is a topic of current interest as several theoretical models were proposed that can be subjected to experimental scrutiny by measuring the fission rate and the time scale for the transition from saddle to scission. We carried out several such studies in which the emission of giant dipole $\gamma$ rays are used as a reference for the fission time scale. By measuring the $\gamma$-ray spectra in

*SUNY at Stony Brook

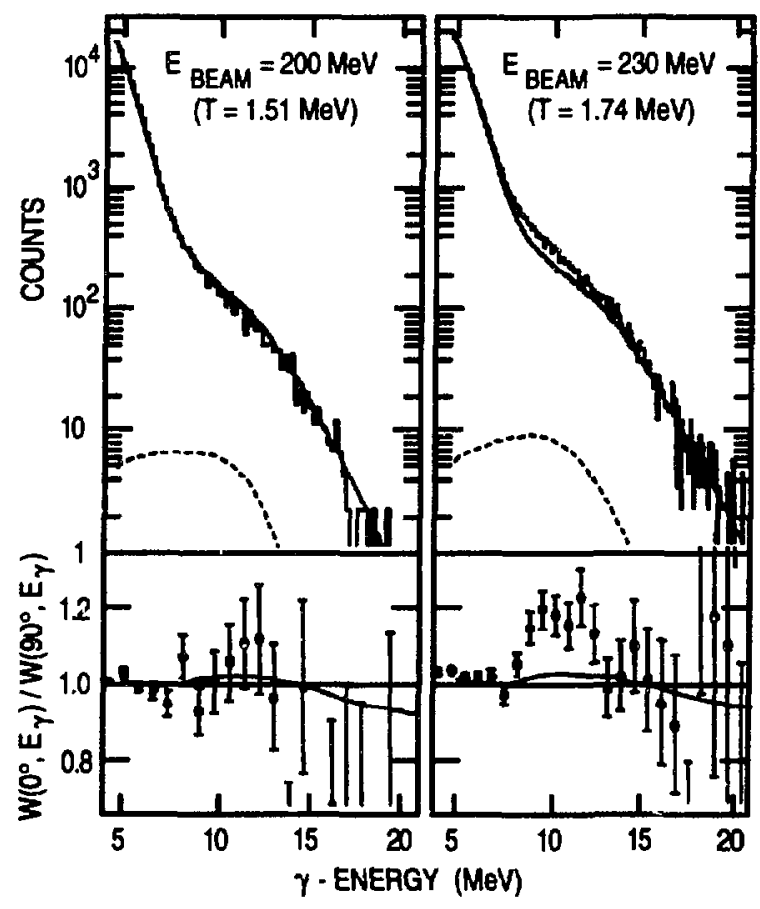

Fig. I-11. Fits to the experimentally measured $\gamma$ ray energy spectra for $200,230 \mathrm{MeV} 32 \mathrm{~S}+208 \mathrm{~Pb}$ without the inclusion of nuclear viscosity. The short dashed line is the pre-saddle yield, the long dashed line is from the subsequent decay of the fragments, the solid line is a sum of these components ad the histogram is the experimental data. The lower two panels show the $\gamma$ fission correlation data (points with error bars) compared to the calculated correlation resulting from the respective fits to the yenergy spectra. The temperatures are calculated using $T=l\left(E^{*}\right.$. $\left.\left.E_{\text {rot }}\right) / a\right]^{1 / 2}$ with $a=A / 8.8$ and the rotational energy $E_{\text {rot }}$ for the average angular momentum using the rigid-sphere moment of inertia. 
coincidence with fission fragments, it is possible to isolate pre-scission $\gamma$ rays on the basis of the spectral shape and angular distribution. From the analysis of several experiments using the reactions ${ }^{16} \mathrm{O}+{ }^{208} \mathrm{~Pb}$ at $\mathrm{E}_{\text {lab }}=100,120,140 \mathrm{MeV},{ }^{32} \mathrm{~S}+{ }^{\text {nat }} \mathrm{W}$ at $\mathrm{E}_{\text {lab }}=185,215,230 \mathrm{MeV}$, and ${ }^{32} \mathrm{~S}+{ }^{208} \mathrm{~Pb}$ at $\mathrm{E}_{\mathrm{lab}}=200,230 \mathrm{MeV}$, the surprising result was found that the contribution from pre-scission GDR $\gamma$ rays increases sharply over a rather narrow excitation energy region from $\mathrm{E}^{*}=$ 50-80 MeV, indicating a corresponding rise in the dissipation strength for the fission process (see Fig. I-11). The value reached at the higher excitation energies agrees well with the predictions from the one-body dissipation model, whereas the value in the low-energy range is consistent with negligible energy dissipation in the fission motion. These results were confirmed in a recent experiment with the ${ }^{32} \mathrm{~S}+{ }^{n a t} \mathrm{~W}$ reaction using beams in the energy range from $\mathrm{E}_{\text {lab }}=185-250$ $\mathrm{MeV}$. The results are published in Phys. Rev. Lett. $\underline{72}, 470$ (1994).

\section{c.6. The General Law of Radioactive Decay Applied to Fission of Hot Nuclei (B. B. Back)}

The law for spontaneous transmutations, such as radioactive decay processes in atomic and nuclear physics, was derived for the general case where the decay rates may be time-dependent. The application to the fission decay of hot nuclei is discussed within a simplified statistical model, with particular emphasis on the emission of neutrons prior to passage of the saddle point as well as during the descent from the saddle to scission (see Fig. I-12). The formalism is expected to have applications in a wide range of problems involving spontaneous transmutations in physics, chemistry and biology. This work will be published in the proceedings of the International School-Seminar on Heavy Ion Physics, Dubna, Russia, May 10-15, 1993.

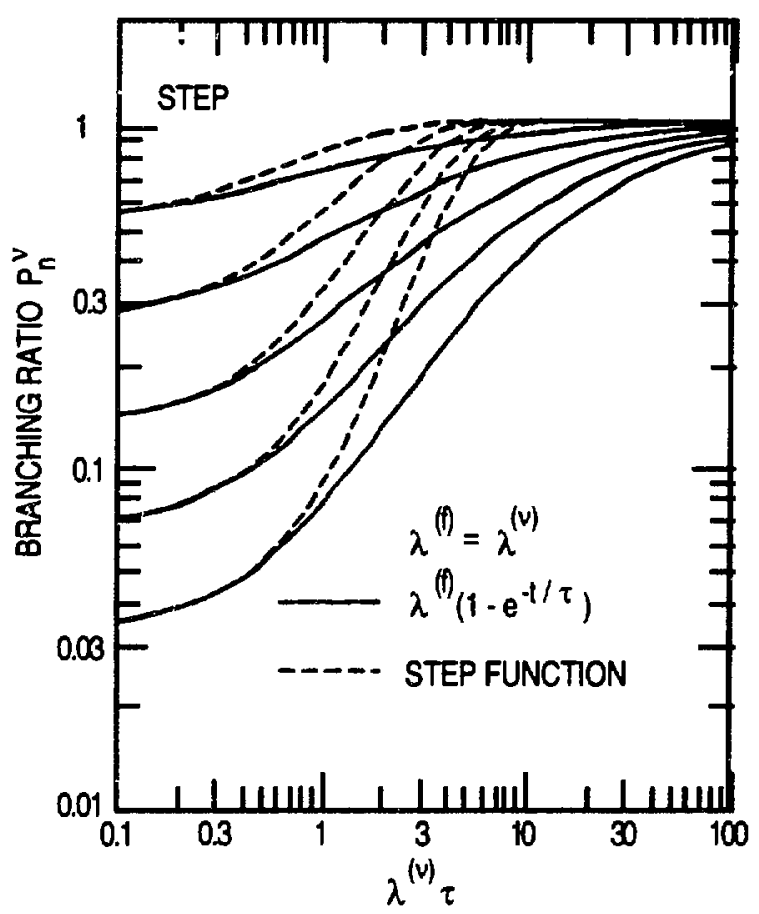

Fig. I-12. Branching ratios $\mathrm{P}^{v}$ for the idealized case with identical decay widths in consecutive steps are shown as a function of the quantity $\lambda^{v} \tau$ for the first through the fifth generation, $n=1-5$, along the decay path with a constant decay width $\lambda^{\nu}$. The solid and dashed curves correspond to a smooth and step function time dependence of the competing decay branch, $\lambda^{f}(t)$, respectively. 


\section{c.7. Evaporation Residue Cross Sections for ${ }^{32} S+{ }^{184} W$ - Energy Dissipation \\ in Hot Nuclei (B. B. Back, C. N. Davids, D. J. Henderson, A. H. Wuosmaa, D. Hofman,* and P. Paul*)}

Since it was suggested recently that the nuclear dissipation strength varies strongly with deformation of the system, it is essential to measure quantities which are sensitive to the dissipation strength in only one of the two deformation regimes, i.e. inside or outside the fission saddle point. It was realized recently that measurements of the evaporation residue cross section in hot nuclei would be very sensitive to the dissipation inside he saddle point only. In this case the dissipation strength manifests itself as an increase in evaporaiion residue cross section over the predictions by the statistical evaporation models. In order to exploit this sensitivity we have started an experimental program to measure evaporation residue cross sections in $32 \mathrm{~S}+184 \mathrm{~W},{ }^{208} \mathrm{~Pb}$ reactions to supplement the GDR $\gamma$-ray measurements performed for the same systems. The Fragment Mass Analyzer (FMA) is ideally suited for such measurements, when using a new detection setup with a double-sided Si strip detector placed $40 \mathrm{~cm}$ behind the focal plane of the instrument. This setup allows for accurate identification of evaporation residues by measuring the time-of-flight and implantation energy of the registered particles. Trial runs to test this method were successfully completed, and a further run to measure the transmission through the FMA and the detection system has been approved.

*SUNY at Stony Brook

c.8. Study of Proton Radioactivities (C. N. Davids, P. J. Woods, * B. B. Back, R. R. Betts, C. R. Bingham, $\nmid$ L. Conticchio, $\ddagger$ M. Freer, $\S$ D. J. Henderson, R. Hermann, C. L. Jiang, R. D. Page,* H. T. Penttilä, A. V. Ramayya,I K. E. Rehm, J. D. Richards, † K. S. Toth, II W. B. Walters, $\ddagger$ A. H. Wuosmaa, and B. E. Zimmerman $\dagger)$

Following the successful commissioning of the implantation facility (see F.a), where protons from the decay of $146,147 \mathrm{Tm}$ were observed, a study of additional cases of proton radioactivity has been initiated. In addition to confirming the decays of ${ }^{109} \mathrm{I},{ }^{151} \mathrm{Lu}$ and ${ }^{160} \mathrm{Re}$, a search for ${ }^{105} \mathrm{Sb}$ was attempted. These data are still under analysis. Figure I-13 shows some preliminary spectra from the study of ${ }^{160} \mathrm{Re}$ decay, illustrating the sensitivity provided by the parent-daughter correlation method. With the combination of the background reduction afforded by the new focusing mode of the FMA (see I.F.a), higher beam intensities and a target lotator, cross-sections at the tens of nanobarn level should be achievable.

*University of Edinburgh. United Kingdom, †University of Tennessee $\$$ University of Maryland. \$University of Birmingham, United Kingdom, TVanderbilt University, llOak Ridge National Laboratory 


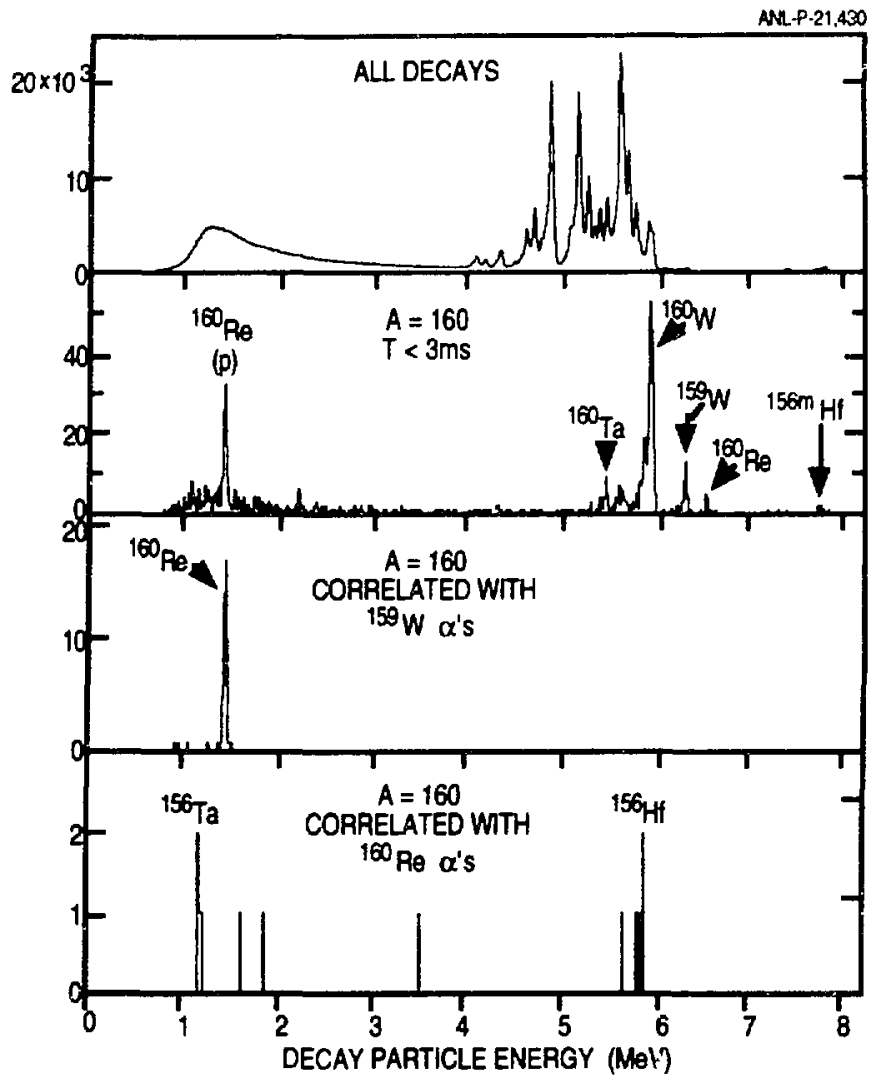

Fig. I-13. (a) All decay events from ions produced in $290-\mathrm{MeV} 58 \mathrm{Ni}+{ }^{106} \mathrm{Cd}$. (b) Decay events occurring within 3 ms of the implantation of an $A=160$ ion. (c) The subset of events in panel (b) which precede the alpha decay of 159 W. (d) The subset of events in panel (b) which follow the alpha decay of ${ }^{160} \mathrm{Re}$.

c.9. Alpha Desay of Heavy Nuclei (C. N. Davids, W. F. Henning, B. B. Back, C. R. Bingham, * L. Conticchio,† D. J. Henderson, R. Hermann, C. L. Jiang, H. T. Penttilä, K. E. Rehm,

Test experiments were performed to study the production of heavy nuclei and the observervation of their subsequent alpha decay in the implantation facility at the FMA. These include the systems ${ }^{58} \mathrm{Ni}+{ }^{116} \mathrm{Sn}\left(\mathrm{Z}_{\text {total }}=78\right),{ }^{92} \mathrm{Mo}+{ }^{92} \mathrm{Mo}\left(\mathrm{Z}_{\text {total }}=84\right)$, and ${ }^{64} \mathrm{Ni}+{ }^{154} \mathrm{Sm}\left(\mathrm{Z}_{\text {total }}=90\right)$. Figure I14 shows a parent-daughter correlation plot observed from the decay of $A=172$ ions from the ${ }^{58} \mathrm{Ni}$ +116 Sn experiment. A new focusing system for the FMA was used (see F.a.) which allows a very clean separation of recoils from the scattered beam background. This means that for the heaviest cases above, where fusion represents $\leq 1 \%$ of the total cross-section, the alpha decays can be studied at the sub-microbarn cross-section level. To reach even heavier systems, cold fusion of ${ }^{64} \mathrm{Ni}$ with $182,184,186 \mathrm{~W}$ and asymmetric combinations of neutron-rich $\mathrm{S}$ and $\mathrm{Ca}$ beams with $\mathrm{W}$, $\mathrm{Pb}, \mathrm{U}$, and $\mathrm{Pu}$ targets will be investigated.

*University of Tennessee, †University of Maryland, ¥Oak Ridge National Laboratory 


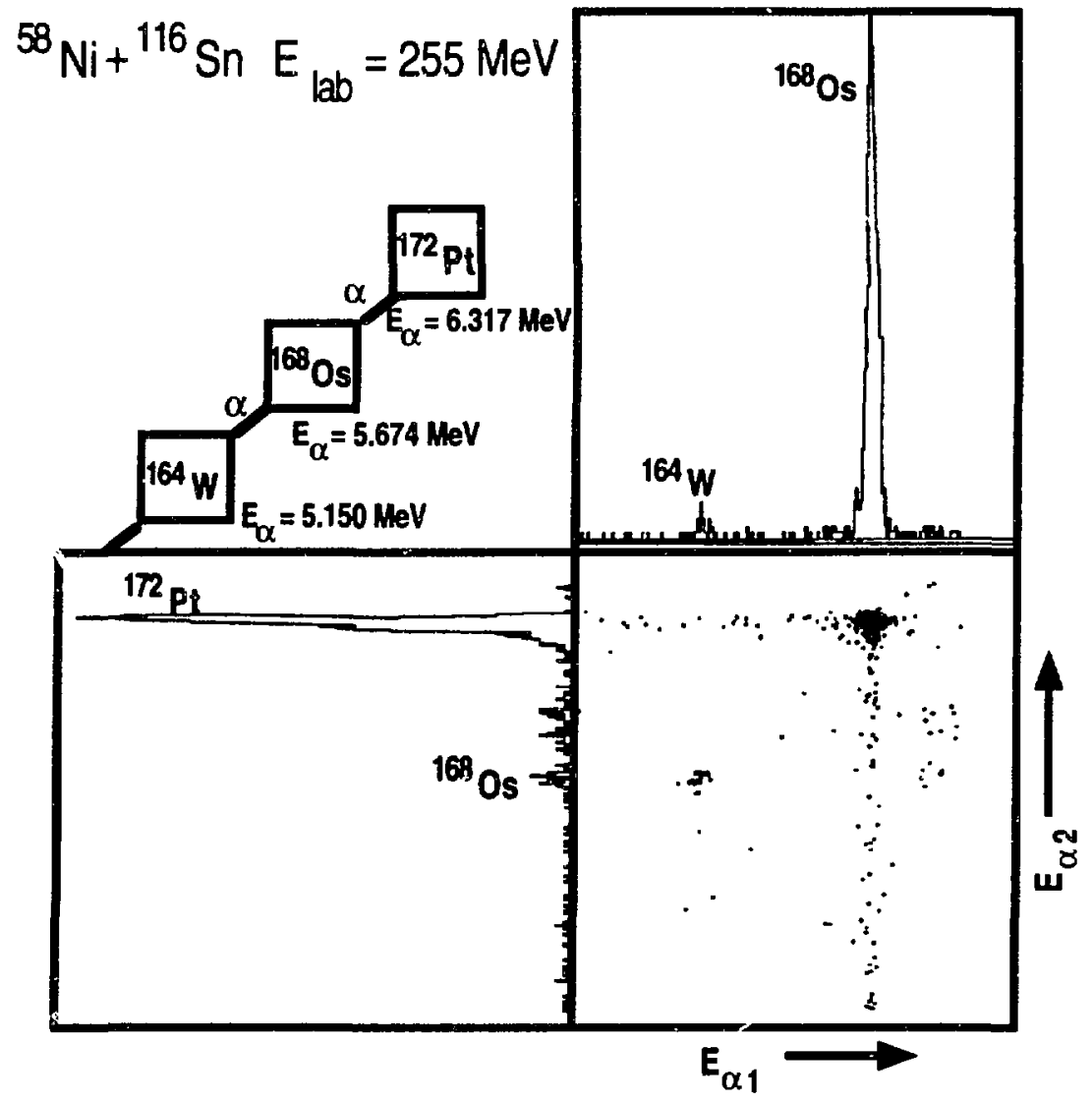

Fig. I-14. Correlation diagram showing parent-daughter relationships between alpha decays following the implantation of $A=172$ ions. 


\section{B. GAMMA-RAY SPECTROSCOPY STUDIES}

This research program focuses on two major areas: (a) studies of nuclear properties associated with very elongated nuclear shapes (superdeformed and hyperdeformed states), and (b) investigations of the evolution of the nuclear shape as a function of proton and/or neutron number as well as a function of angular momentum and temperature (excitation energy above the yrast line).

A major focus of the Argonne work is on the study of superdeformation, with particular emphasis on properties associated with this phenomenon in the A $\sim 190$ region. First evidence for the existence of this region of superdeformation was obtained at Argonne five years ago. Recently, first attempts were made to identify hyperdeformed shapes in the A $~ 180$ region. The data collected during these superdeformation studies also provide excellent opportunities to perform investigations of other structure phenomena of interest. In particular, such detailed studies allow objective (b) outlined above to be addressed.

New large $\gamma$-ray detector arrays are currently under construction in the United States (GAMMASPHERE) (GS) and in Europe (EUROGAM). These new arrays provide new opportunities for nuclear structure research. Argonne is participating vigorously in the construction of GAMMASPHERE and has performed experiments with the so-called "early implementation phase" of the device in 1993. The group is also collaborating in two experiments at EUROGAM. Firsi results from these experiments show clearly the promise of these devices. They are discussed below.

A number of projects described in this section took advantage of the capabilities of the ArgonneNotre Dame BGO $\gamma$-ray facility which consists of 50 hexagonal BGO detectors (used mainly as a sum-energy/multiplicity filter) surrounded by 12 Compton-suppressed Ge detectors. A wide variety of computer programs are available from the collaboration which provide assistance with setting up the experiments (gain matching, adjustment of constant-fraction discriminators, offset settings on ADCs, etc.) and with data reduction and analysis (sorting of the events into $\gamma-\gamma$ coincidence matrices under a variety of gating conditions, peak fitting routines, etc.). A scattering chamber for coincidence measurements between $\gamma$ rays and particles identified by either $\Delta E-E$ or time-of-flight techniques is also available. This equipment has been constructed by the University of Kansas group. A plunger apparatus for recoil-distance measurements of nuclear lifetimes has been developed by the Notre Dame group. The device fits entirely inside the BGO array. Dedicated chambers have been constructed for other experiments as well ( $\mathrm{g}$-factor measurements, fission-fragment coincidence measurements, etc.). Support for up to 7 Compton-suppressed spectrometers at the magnetic spectrograph is also available. Ten of the Compton-suppressed spectrometers can also be located at the target position of the Fragment Mass Analyzer (FMA). A special scattering chamber for use of the $\gamma$-ray detectors at the FMA is available as well as a dedicated electronics setup.

Several projects are joint efforts with groups from Lawrence Berkeley and Lawrence Livermore Laboratories, the University of Notre Dame, Purdue University, INEL-Idaho Natıonal Engineering Laboratory, North Carolina State University, the Australian National University, the University of Manchester, the University of Tennessee, and Yale University. The work at EUROGAM was performed in collaboration with several other European laboratories.

\section{a. States in the Superdeformed Secondary Minimum and Their Coupling with States in the Normal Well}

i. Introduction

The occurrence of an excited secondary minimum at large deformation provides a rare opportunity to study states which are cold, although highly excited with respect to the normal yrast line. 
Within the superdeformed well, isolated from normal states with smaller deformation, there is a cold "ground" state, as well as low-lying excited states which can give rise to sharp equally-spaced transitions. With increasing excitation energy, the coupling with states outside the well grows until the separate identity of SD states melts away; in addition, the collective properties may be altered. When the SD "yrast" state lies high enough above the true (normal) vrast state, then a coupling occurs between a cold system with a hot normal one, causing the SD band to decay.

Research on superdeformation at Argonne addresses the physics associated with states within the SD well and their coupling with states outside the well. Investigation of the decay out of SD states into lower-lying normal states allows us to examine the coupling between a cold, ordered system and a hot, chaotic one. This problem is also of general interest outside of nuclear physics. Discrete line spectroscopy investigates the cold SD bands. Study of the feeding of SD bands and of the associated quasicontinuum $\gamma$ rays probes the nature of excited states and their increasing mixing with normal states.

\section{ii. Decay of SD Bands}

The sudden decay of SD bands after a long series of up to 20 intraband lines is a striking phenomenon. To understand it, many experimental attempts were made but were not successful. We used a novel approach to address this long-standing problem. Instead of trying to decipher the fragmented decay pathways, the complete spectrum of $\gamma$ rays decaying out of the SD band was measured. We wire able to characterize the decay mechanism, and define experimental excitation energies and spins for a SD band in ${ }^{192} \mathrm{Hg}$. The complete spectrum of the $\gamma$ rays linking states in two separate wells was extracted. This spectrum, which has a quasicontinuous distribution with superposed broad structures (in particular a conspicuous bump between 1.4 and $2.2 \mathrm{MeV}$ ) and sharp peaks, establishes the decay mechanism as due to mixing of a SD state with some of the sea of normal states in which it is embedded. We performed calculations with this picture and can reproduce the sudden drop in transition intensity, as well as all the features of the decay spectrum. The calculations suggest that the clustering of $\gamma$ strength between 1.4 and $2.2 \mathrm{MeV}$ may be due to a perturbation of the level densities due to pairing, which may allow to probe the dissolution of pairing with temperature. The $\gamma$ spectra associated with the different stages of a $\gamma$ cascade which flows through SD bands show that the nucleus undergoes an unusual transition: chaos-orderchaos-order. It also demonstrates that the decay is a result of coupling a cold ordered system with a hot chaotic one. The spectra also reveal that when decay takes place the average number of steps from the SD state to the normal yrast line is $3.2 \pm 0.6$, the mean excitation energy above yrast is $4.3 \pm 0.9 \mathrm{MeV}$, and the mean spin is $10.1 \pm 0.5$.

We developed techniques for analyzing coincirences involving quasicontinuum $\gamma$ rays, and learned that the pronounced bump structure probably arises from a last-step transition to the vicinity of the yrast line.

\section{iii. Cold States}

Twenty three SD bands in the mass 190 region were found from work done at ATLAS, GAMMASPHERE and EUROGAM. This large body of data was vital in helping to identify the occurrence of "identical" bands, i.e. SD bands inneighboring nuclei which have transition energies with $\Delta E<1 / 500$, or which have identical dynamic moments of inertia $\mathfrak{I}^{(2)}$. The identical bands, which were not anticipated, are still not explained, but may imply a symmetry which has yet to be identified. We established which general class of orbitals give identical bands (natural parity orbitals which are flat as a function of frequency) and which ciasses do not (orbitals from two major shells above which plunge down with deformation). The latter generally exhibit alignment, which gives rise to the almost universal rise in $\mathfrak{I}^{(2)}$ observed in SD bands with $A \sim 190$. At the highest frequencies, we observed either a flattening of $\mathfrak{I}^{(2)}$ (in ${ }^{192} \mathrm{Hg}$ ) or a downturn (in ${ }^{194} \mathrm{Hg}$ ), which are predicted to occur after alignment is complete. However, when these orbitals are 
blocked, the $\mathfrak{I}^{(2)}$ is almost constant, as we first demonstrated in ${ }^{192} \mathrm{Tl}$. The presence of alignment also implies the persistence of pauring at large deformation and spin. We measured lifetimes of individual states of SD bands in $192,194 \mathrm{Hg}$ which prove that the deformation is indeed large and that it is stable with respert to spin and particle excitation.

Identification of vibrational states in the SD well can serve to establish the rigidity of the deformation with respect to $\beta, \gamma$ or octupole distortions. Theory has pointed out that SD nuclei may manifest octupole instability. The first indications for an octupole SD vibrational band in ${ }^{190} \mathrm{Hg}$ were found (from GAMMASPHERE data) at a surprisingly low energy $(\sim 600 \mathrm{keV})$ above the yrast SD band. Finally, we discovered a band in ${ }^{151} \mathrm{Dy}$, with energies midway between those in ${ }^{152} \mathrm{Dy}$, which provides additional evidence for pseudospin symmetry.

iv. Excited SD States

From EUROGAM and GAMMASPHERE data it was established that excited SD bands give rise to a pronounced E2 bump in the ' $y$ spectrum. This feature allows to probe the collective properties of excited SD states. There rse preliminary indications that the quadrupole moment and moment of inertia of the excited SD scates are larger than those of the yrast SD band in ${ }^{192} \mathrm{Hg}$. The coupling of excited SD and ND states and the mechanism for the unexpectedly large population of SD states have been studied by both experiment and theory. We are able to reproduce by Monte Carlo simulations all observables connected with the feeding: band intensities, variation of intensity with spin, entry distribution (in spin and energy) of states leading to trapping in the SD well, and the spectra of feeding $\gamma$ rays. In the process we set constraints on the energy of the SD band $(U=3.3-$ 4.3 MeV above normal yrast line at spin 10) and the well depth (3.5-4.5 MeV at spin 40).

\section{a.1. Spectrum of $\gamma$ Rays Connecting Superdeformed and Normal States in ${ }^{192} \mathrm{Hg}$}

(R. G. Henry, T. Lauritsen, T. L. Khoo, M. P. Carpenter, R. V. F. Janssens, I. Ahmad,

F. Hannachi,* I. Deloncle,* B. Gall,* M. G. Porquet,* C. Schuck,* G. Smith,*

R. Beraud,$\dagger$ Y. Lecoz, $\dagger$ M. Meyer,$\dagger$ N. Redon, $\dagger$ F. Azaiez, $\ddagger$ C. Bourgeois, $\ddagger$ J. Duprat $\neq$

A. Korichi, $\ddagger$ N. Perrin $\ddagger$ H. Sergolle $\ddagger$ H. Hubel,§ P. Willsau,§ J. Sharpey-Schafer,,

C. Beausang, I E. Paul, ( M. Joyce, I B. Wadsworth,\| R. Clark,\|l and J. Simpson**)

Almost a hundred superdeformed bands have been found in the mass 150 and 190 regions. Nevertheless, the energies and spins of the SD levels have still not been measured (with one possible exception). Many attempts have been made to decipher the highly fragmentedpathways connecting SD and normal states, but with hitherto no success. We adopted a new approach that consists of characterizing the overall spectral shape of the $\gamma$ rays linking SD and normal states.

We detected $\gamma$ rays from the ${ }^{160} \mathrm{Gd}\left({ }^{36} \mathrm{~S}, 4 \mathrm{n}\right){ }^{192} \mathrm{Hg}$ reaction using EUROGAM, a detector array with 43 Compton-suppressed Ge detectors. Pairwise gates were set on known SD transitions in ${ }^{192} \mathrm{Hg}$ in order to obtain the coincident $\gamma$-ray spectrum. Gamma rays that precede and follow the SD band can be distinguished on the basis of their Doppler shifts. However, the spectrum of statistical feeding transitions is broad and difficult to extricate by means of the Doppler shift alone; thus it was given by a calculation. We succeeded in isolating the spectrum of $\gamma$ rays linking SD and "normal" states. The spectrum is comprised of sharp lines and a quasicontinuous component, which has interesting structure. The most striking (and surprising) feature is a broad bump between 1.4 and $2.2 \mathrm{MeV}$ [see Fig. I-15(c)]. This spectrum reveais the mechanism for decay out of the SD well: it arises from coupling between a SD state and the sea of normal states in which it

\footnotetext{
*Centre de Spectrometrie Nucleaire et de Spectrometrie de Masse, Orsay, France, $\nmid$ Universite Claude Bernard, Lyon, France, ¥Institut de Physique Nucleaire, Orsay, France, §Universität Bonn, Germany, TUniversity of Liverpool, United Kingdom, IUUniversity of York, United Kingdom, **SERC Daresbury Laboratory, United Kingdom
} 
is embedded. The spectrum indicates that the decay from the SD state to the normal yrast line takes $3.2 \pm 0.6$ steps, that the excitation energy of the SD state above yrast is $4.3 \pm 0.8 \mathrm{MeV}$, and that the SD state from which the predominant decay takes piace has spin $10.1 \pm 0.5 \hbar$.

The different components of the spectrum associated with the formation and decay of SD states [see Fig. I-15(a-d)] reveals an unusual sequence of events, with a transition from chaos to order to chaos to order. A sudden transition from regularly-spaced SD lines to a broad thermal decay spectrum shows that the decay is a result of coupling a cold, ordered, SD system with a hot, chaotic, normal one.

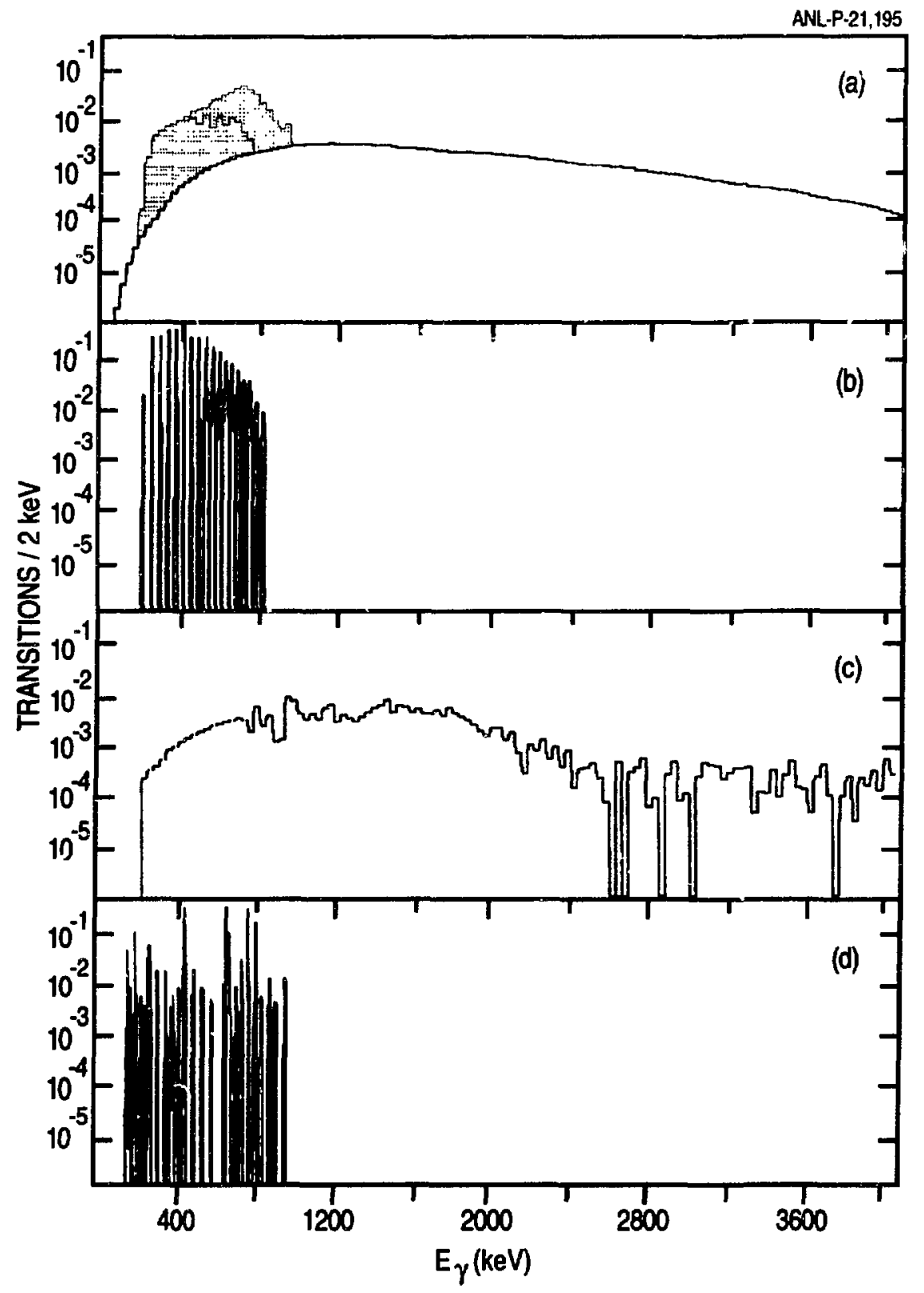

Fig. I-15. Spectra of $\gamma$ rays emitted at different stages in the feeding and decay of the SD band in ${ }^{192} \mathrm{Hg}$. (a) transitions feeding the SD band -- calculated statistical feeding spectrum and measured $E 2$ and $M 1 / E 2$ peaks from transitions between excited states in the SD well; $(b)$ regularly spaced E2 peaks. from the yrast (cold) SD bard; (c) spectrum of $\gamma$ rays linking SD and ND states, showing features characteristic of a hot system; and (d) sharp lines from ND nearyrast states populated in the decay of SD states. 


\section{a.2. New Techniques of Quasicontinuum Spectroscopy for Investigating the Decay Out of Superdeformed Bands (T. L. Khoo, T. Lauritsen, T. Dфssing, R. G. Henry, C. Tan,* I. Ahmad, M. P. Carpenter, B. Crowell, and R. V. F. Janssens)}

The spectrum of $\gamma$ rays decaying out of the superdeformed band in ${ }^{192} \mathrm{Hg}$ has a quasicontinuous distribution. Whereas methods to construct level schemes from discrete lines in coincidence spectra are well established, new techniques must still be developed to extract information from coincidences involving quasicontinuous $\gamma$ rays. From an experiment using EUROGAM, we obtained impressively clean 1 - and 2-dimensional $\gamma$ spectra from pairwise or single gates, respectively, on the transitions of the superdeformed (SD) band in ${ }^{192} \mathrm{Hg}$. We investigated methods to exploit the 2-dimensional quasicontinuum spectra coincident with the SD band to determine the excitation energy of the SD band above the normal yrast line. No strong peaks were observed in the 2- $\gamma$ sum spectra; only candidates of peaks at a 2-3 $\sigma$ level were found. This suggests that $2-\gamma$ decay is not the dominant decay branch out of SD bands, consistent with the observed multiplicity of 3.2 (see B.a.1.).

Our measurements of the spectrum of $\gamma$ 's following decay out the SD band in ${ }^{192} \mathrm{Hg}$ (see B.a.1.), reveal a , rominent bump around $1.6 \mathrm{MeV}$. Spectra from gates set on high-energy peaks show a conspicuous absence of the bump. Sharp high-energy lines can arise only from transitions to the immediate vicinity of the yrast line, since it is only here that the level density is sufficiently low. Hence, transitions in the bump must proceed parallel to the high-energy lines, and hence, comprise the last steps of decay to the yrast region.

\section{a.3. Calculations of the Decay Out of SD Bands (T. Lauritsen, T. L. Khoo, T. Dфssing, R. G. Henry, I. Ahmad, M. P. Carpenter, B. Crowell, and R. V. F. Janssens)}

We advocated that the decay out of SD bands to the lower-lying normal states arises because of statistical mixing with the sea of hot dense normal states in which the SD state is embedded. Using this model, the intensities of individual SD transitions and the variation of observed intensities with spin are reproduced. The sudden decrease of intensity at low spin requires that the barrier separating SD and normal states decreases with lowering spin, although it is still $\sim 1 \mathrm{MeV}$ above the SD minimum at the point of decay.

In this model, the $\gamma$ decays which connect SD and normal states should have a statistical-like spectral distribution (together with superposed sharp lines and broad features, which reflects the fact that the full statistical limit is not achieved). The spectrum is, in fact, that emitted by a highlyexcited normal state (which mixes with the SD state). We calculated this spectrum by a Monte Carlo simulation of the statistical cascade and with an analytic expression for the decay spectrum. The calculated spectra have a strong similarity with the measured spectrum for decay from the ${ }^{192} \mathrm{Hg}$ SD band (see B.a.1.): both the multiplicity and spectral shape are reproduced. We plan to perform further calculations using improved level densities.

\section{a.4. Calculation of the Spectrum of $\gamma$ Rays Connecting Superdeformed and Normally Deformed Nuclear States (T. Dфssing, T. L. Khoo, T. Lauritsen, I. Ahmad, M. P. Carpenter, B. Crowell, R. G. Henry, and R. V. F. Janssens)}

The decay out of superdeformed bands may occur by coupling to compound nuclear states of normal deformation. The decay caused by this coupling should be statistical electromagnetic nuclear decay, initiated at one or a few states of a rotating nucleus. Investigating the spectra of $\gamma$ rays of this decay in the mass regions $A=150$ and 190 will shed light on the properties of otherwise inaccessible excited nuclear states. 
Our first description of the $\gamma$-ray spectrum focuses on the pairing correlations, applying a schematic many-quasiparticle BCS level spectrum, and also schematic statistical decay matrix elements. The modification of the level density caused by the gradual weakening of pairing correlations gives rise to a broad bump in the statistical decay spectrum, resembling recent experimental results for decay from a SD band in ${ }^{192} \mathrm{Hg}$. In contrast to this, monotonous and featureless spectra are obtained in the absence of pairing. More detailed properties of the emitted $\gamma$ rays, such as $E_{\gamma} E_{\gamma}$ coincidence spectra and fluctuations of the spectra, are found to further elucidate the pairing correlations.

We are presently aiming at giving the results a more general foundation, by also investigating level schemes from a number of projected BCS states, by comparing grand canonical treatments of the pairing with level counting, and by applying more realistic cranked BCS level schemes.

\section{a.5. Spectra of $\gamma$ Rays Feeding Superdeformed Bands (T. Lauritsen, T. L. Khoo,

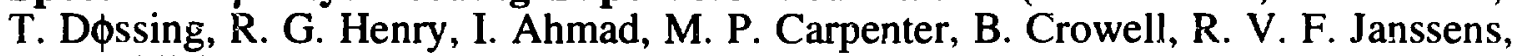 and D. Nisius)}

The spectrum of $\gamma$ rays coincident with SD transitions contains the transitions which populate the SD band. This spectrum can provide information on the feeding mechanism and on the properties (moment of inertia, collectivity) of excited SD states. We used a model developed to explain the feeding of SD bands to calculate the spectrum of feeding $\gamma$ rays. The Monte Carlo simulations take into account the trigger conditions present in our EUROGAM experiment (see B.a.1.). Both experimental and theoretical spectra contain a statistical component and a broad E2 peak (from transitions occurring between excited states in the SD well). There is good resemblance between thie measured and calculated spectra. Work is continuing to improve the quality of the fits, which will result in a better understanding of excited SD states. In addition, a model for the last steps, which cool the $\gamma$ cascade into the SD yrast line, needs to be developed. A strong M1/E2 lowenergy component, which was also observed, is believed to be responsible for this cooling.

\section{a.6. Decay from the Superdeformed Bands in ${ }^{194} \mathrm{Hg}$ (M. P. Carpenter, I. Ahmad,} S. Harfenist, R. G. Henry, R. V. F. Janssens, T. L. Khoo, T. Lauritsen, J. R. Hughes,* J. A. Becker,* M. J. Brinkman, ${ }^{*}$ B. Cederwall, $†$ M. A. Deleplanque, $\dagger$ R. M. Diamond, $†$ J. E. Draper, $\ddagger$ C. Duyar $\ddagger$ P. Fallon, $†$ E. A. Henry,* R. W. Hoff,* I. Y. Lee, $\dagger$ E. Rubel, $\neq$ F. S. Stephens, $†$ and M. A. Stoyer*)

Superdeformed bands in ${ }^{194} \mathrm{Hg}$ were studied using the early implementation of GAMMASPHERE. The response functions for the Ge detectors were measured for the first time as part of this experiment. Experiments were performed with both a backed target (where the residue stopped in the $\mathrm{Au}$ backing) and a thin target (where the residue recoiled into vacuum). This will permit measurements of the decay times of the quasicontinuum $\gamma$ rays. Preliminary analysis of the spectrum in coincidence with the yrast SD band in ${ }^{194} \mathrm{Hg}$ reveals the same features as found in the quasicontinuum structure in ${ }^{192} \mathrm{Hg}$ (see B.a.l.). These features include: statistical $\gamma$ rays feeding the SD band, a pronounced E2 peak from transitions feeding the SD band, a M1/E2 bump at low energies that is associated with the last stages of feeding of the superdeformed band, and a quasicontinuous distribution from $\gamma$ rays linking SD and normal states, including a sizable clustering of strength around $1.7 \mathrm{MeV}$. The similarity of the decay spectra suggests a common decay mechanism applies in these two cases, and probably in other cases where the SD band is high'y excited. The analysis is ongoing.

*Lawrence Livermore National Laboratory, $\nmid$ Lawrence Berkeley Laboratory, $\ddagger$ University of California, Davis 
a.7. Moment of Inertia at the Highest Spins in ${ }^{192} \mathrm{Hg}$ (R. G. Henry, T. Lauritsen, T. L. Khoo, M. P. Carpenter, R. V. F. Janssens, I. Ahmad, F. Hannachi,* I. Deloncle,* B. Gall, ${ }^{*}$ M. G. Porquet,* C. Schuck,* G. Smith,* R. Beraud, $\dagger$ Y. Lecoz, $\dagger$ M. Meyer, $\dagger$ N. Redon, $†$ F. Azaiez,$\ddagger$ C. Bourgeois, $\ddagger$ J. Duprat $\ddagger$ A. Korichi,$\ddagger$ N. Perrin, $\ddagger$

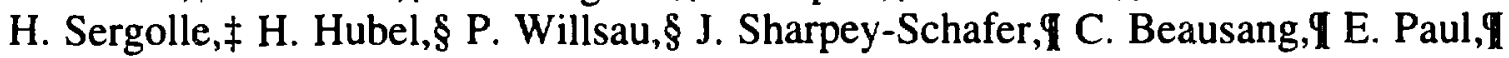
M. Joyce,I B. Wadsworth,II R. Clark,Il and J. Simpson**)

The dynamic moments of inertia $\mathfrak{I}(2)$ for almost all superdeformed (SD) bands in the $A=190$ region show a monotonic increase. We showed that particle alignment is responsible for this rise. If this is inceed the correct explanation, then the $\mathfrak{I}(2)$ should begin to turn down at the highest spins when allignment is complete. Our earlier data from ATLAS showed a continued rise in $\mathfrak{I}(2)$, which was not consistent with this expectation. New data with much better statistics from a EUROGAM experiment, however, show that the energies of the two highest transitions need to be revised. At the highest frequencies, a flattening of $\mathfrak{S}(2)$ is now observed (see Fig. I-16). Results of Hartree-Fock Bogoliubov calculations also show a general rise and flattening of $\mathfrak{I}(2)$.

${ }^{*}$ Centre de Spectrometrie Nucleaire et de Spectrometrie de Masse, Orsay, France, †Universite Claude Bernard, Lyon, France, $\ddagger$ Institut de Physique Nucleaire, Orsay, France, §Universität Bonn, Germany, TUniversity of Liverpool, United Kingdom, IIUniversity of York, United Kingdom, **SERC Daresbury Laboratory, United Kingdom

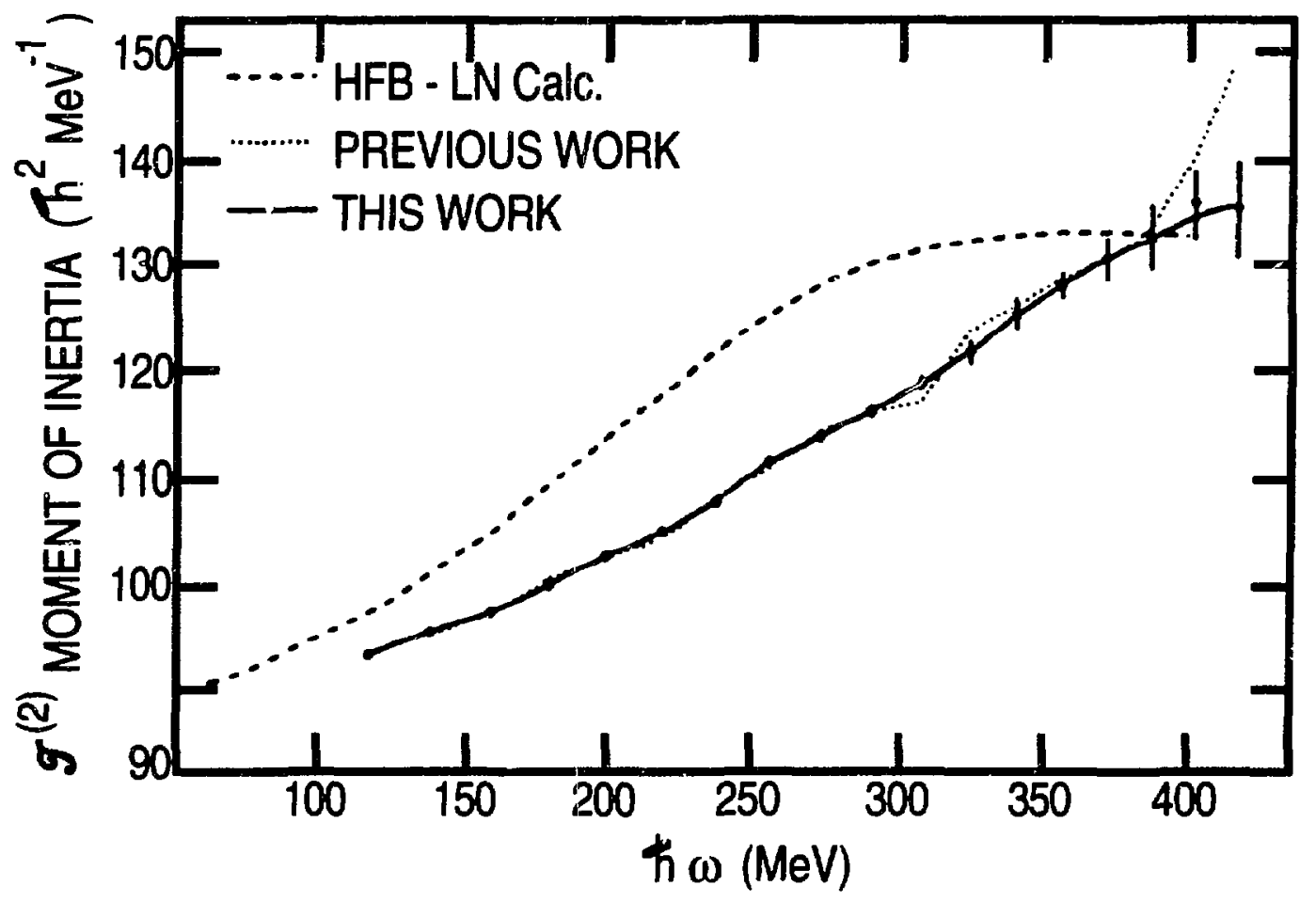

Fig. I-16. Comparison between the new experimental values (solid line), previous experimental values (dotted line) and HFB -LN calculations (dashed line) of the $\mathfrak{I}^{(2)}$ dynamical moment of inertia as a function of rotational frequency. 
a.8. Excited Superdeformed Bands in ${ }^{192} \mathrm{Hg}$ (T. Lauritsen, T. L. Khoo, I. Ahmad, M. C. Carpenter, B. Crowell, R. G. Henry, R. V. F. Janssens, D. Nisius, P. Fallon,* C. Schuck, $\uparrow$ B. Gall, $\uparrow$ F. Hannachi, $\uparrow$ and A. Korichi†)

Large shell gaps are calculated to exist for both protons and neutrons in ${ }^{192} \mathrm{Hg}$ at large deformation. This is supported by the fact that only one SD band was found in this nucleus. Thus, it is of interest to search for excited SD bands in this nucleus, with the eventual goal of determining their excitation energies to provide a measure of the shell gaps. With the operation of the new generation gamma-detector arrays, GAMMASPHERE and EUROGAM, it is now possible to detect weak excited bands in the superdeformed secondary minimum.

We performed an experiment on the nucleus ${ }^{192} \mathrm{Hg}$ using a thin target at GAMMASPHERE in its early implementation phase. An excited superdeformed band was found in this experiment, confirming preliminary suggestions from a EUROGAM experiment with a thick target. Unlike most SD bands in the mass 190 region that have a dynamical moment of inertia which rises monotonically with frequency, that of the excited band has a sharp rise and a fall. This is indicative of a band crossing. There are also tentative indications for another excited SD band. The interpretation of the data is still in progress.

$\overline{* L a w r e n c e ~ B e r k e l e y ~ L a b o r a t o r y, ~ † C S N S M, ~ O r s a y, ~ F r a n c e, ~} \stackrel{\ddagger}{\ddagger}$ IPN, Orsay, France

\section{a.9. Inter-Band Coincidences in the Superdeformed Well of ${ }^{190} \mathrm{Hg}$ (B. Crowell,} M. P. Carpenter, R. V. F. Janssens, R. G. Henry, I. Ahmad, T. L. Khoo, T. Lauritsen, and D. T. Nisius)

Very few experimental observables are ordinarily accessible for superdeformed. (SD) states in the A $\sim 150$ and $A \sim 190$ regions. The gamma-decay out of the superdeformed bands usually proceeds directly to the normally deformed states, through highly fragmented pathways, making it difficult to determine the spins, parities and excitation energies of the SD states. The in-band E2 transitions are so collective $\left(2 \times 10^{3}\right.$ single-particle units in the $A \sim 190$ region $)$ that it is typically impossible to detect any of the competing M1 and E1 transitions between states in the SD well.

In two recent experiments at GAMMASPHERE, we detected an excited SD band in the nucleus ${ }^{190} \mathrm{Hg}$ which decays to the previously observed vacuum SD band in that nucleus, (see Fig. I-17) rather than decaying into the sea of highly excited normally deformed states, as is the case for all other known SD bands in the $A \sim 150$ and $A \sim 190$ regions. Gamma-ray coincidence relationships are observed between the two bands which show that the depopulation of the excited band occurs gradually, over the range of rotational frequencies between 0.25 and 0.30 $\mathrm{MeV}$. The moment of inertia of the excited band displays a behavior which is unique for an eveneven nucleus in the $A \sim 190$ region of super-deformation, maintaining a constant value of $\mathfrak{I}^{(2)}=$ $125 \mathrm{MeV}^{-1}$ throughout the entire observed range of angular frequencies, from 0.25 to $0.35 \mathrm{MeV}$. All other even-even nuclei in the region show $\mathfrak{I}^{(2)} \approx 90-100 \mathrm{MeV}^{-1}$ at low spins, with a marked increase continuing up to the highest spins observed. Even more intriguing is the recent prediction, using generator coordinate calculations, of the characteristics of the one-phonon octupole-vibrational states in the superdeformed well. Extremely strong E1 transitions are predicted to exist between the one-phonon and zero-phonon bands, and quadrupole-octupole coupling effects are predicted to be irinportant, possibly accounting for the unprecedentedly large moment of inertia of the excited band we observed. 


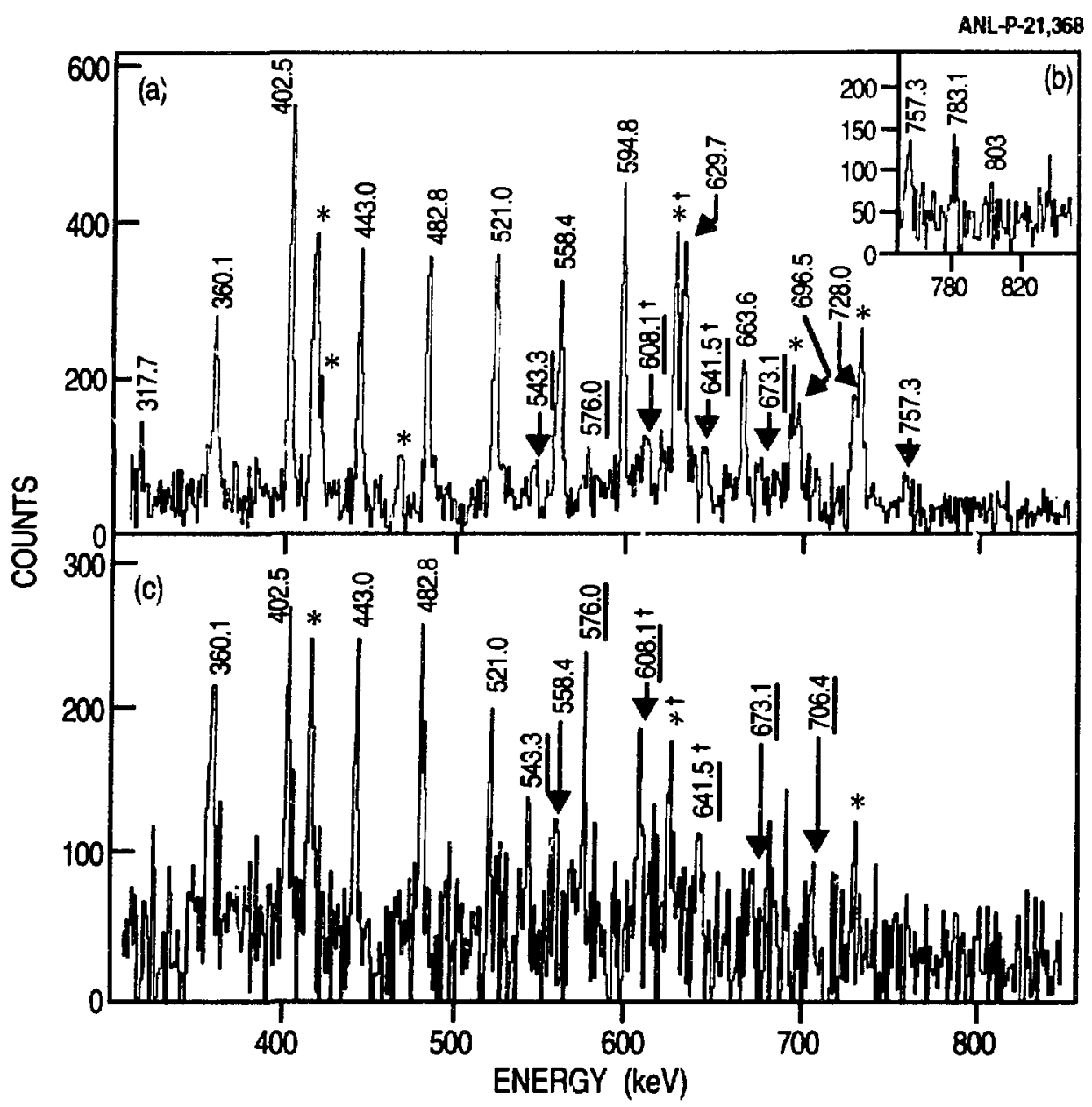

Fig. 1-17. Double-gated gamma-ray coincidence spectra, showing SD transitions in $190 \mathrm{Hg}$ :

(a) sum of double gates on transitions in the yrast SD band with $\hbar \omega<0.28 \mathrm{MeV}$; (b) similar to panel a, but with gates at high frequencies to enhance the top of the band; (c) sum of double gates on transitions in the excited SD band. Transitions in the excited SD band are marked with underlining, normally deformed transitions in coincidence with the SD bands are marked with asterisks, and transitions which are doublets with the proposed interband E1 transitions are marked with daggers. Areas of peaks do not correspond to actual gamma-ray intensities because some transitions have been used as gates, while others have not because they are unresolved doublets.

We also extended the vacuum SD band to higher and lower rotational frequencies, and detected a sharp increase in the moment of inertia at $\hbar \omega=0.38 \mathrm{MeV}$. This apparent observation of a bandcrossing is in good agreement with the most recent Hartree-Fock calculations.

We have tentatively located the actual discrete transitions which connect the two bands. These transitions proved elusive in the original GAMMASPHERE dataset, but were detectable with the greatly increased statistics ( 6 times greater) provided by the second experiment. Unfortunately, these $\gamma$ rays are close in energy to other transitions in ${ }^{190} \mathrm{Hg}$, making a firm assignment difficult. 
a.10. Spectroscopy of the Superdeformed Band in ${ }^{196} \mathrm{~Pb}$ (Y. Liang,

R. V. F. Janssens, M. P. Carpenter, I. Ahmad, R. G. Henry, T. L. Khoo, T. Lauritsen,

E. F. Moore, * H. L. Harrington, * I. G. Bearden, P. J. Daly, $\ddagger$ B. Fornal, $\ddagger$

Z. W. Grabowski, $¥$ R. H. Mayer, $\ddagger$ D. Nisius, $\ddagger$ M. Sferrazza, $\ddagger$ M. W. Drigert,$\S$

U. Garg,I and W. ReviolI)

The study of superdeformation (SD) in the $\mathrm{Pb}$ isotopes was undertaken with the following general motivations: (1) calculations for the $\mathrm{Pb}$ nuclei show rather strong octupole effects for which experimental evidence is currently at best rather weak; (2) finding excited SD bands in these isotopes would help to elucidate questions regarding the neutron and proton single-particle energies in the vicinity of the Fermi surface at large deformations, (3) the possible discovery of so-called "identical" SD bands, i.e. bands with transition energies essentially identical to those observed for superdeformed bands in neighboring nuclei, would allow us to test some of the explanations proposed to account for this unexpected phenomenon, (4) small changes in the deformation are expected with respect to ${ }^{192} \mathrm{Hg}$, the calculated doubly-magic SD nucleus.

We used the ${ }^{170} \operatorname{Er}\left({ }^{30} \mathrm{Si}, 4 \mathrm{n}\right)$ reaction to study the ${ }^{196} \mathrm{~Pb}$ nucleus with ATLAS beams of 142,146 and $155 \mathrm{MeV}$. Experiments were performed with thin targets, where the evapr, ration residues recoil into vacuum, as well as with $\mathrm{Pb}$-backed targets in which the recoiling nuclei are slowed down and lifetime information can be obtained with the DSAM (Doppler Shift Attenuation Method).

*North Carclina State University, $¥$ Purdue University, §Idaho National Engineering Laboratory, TUniversity of Notre Dame

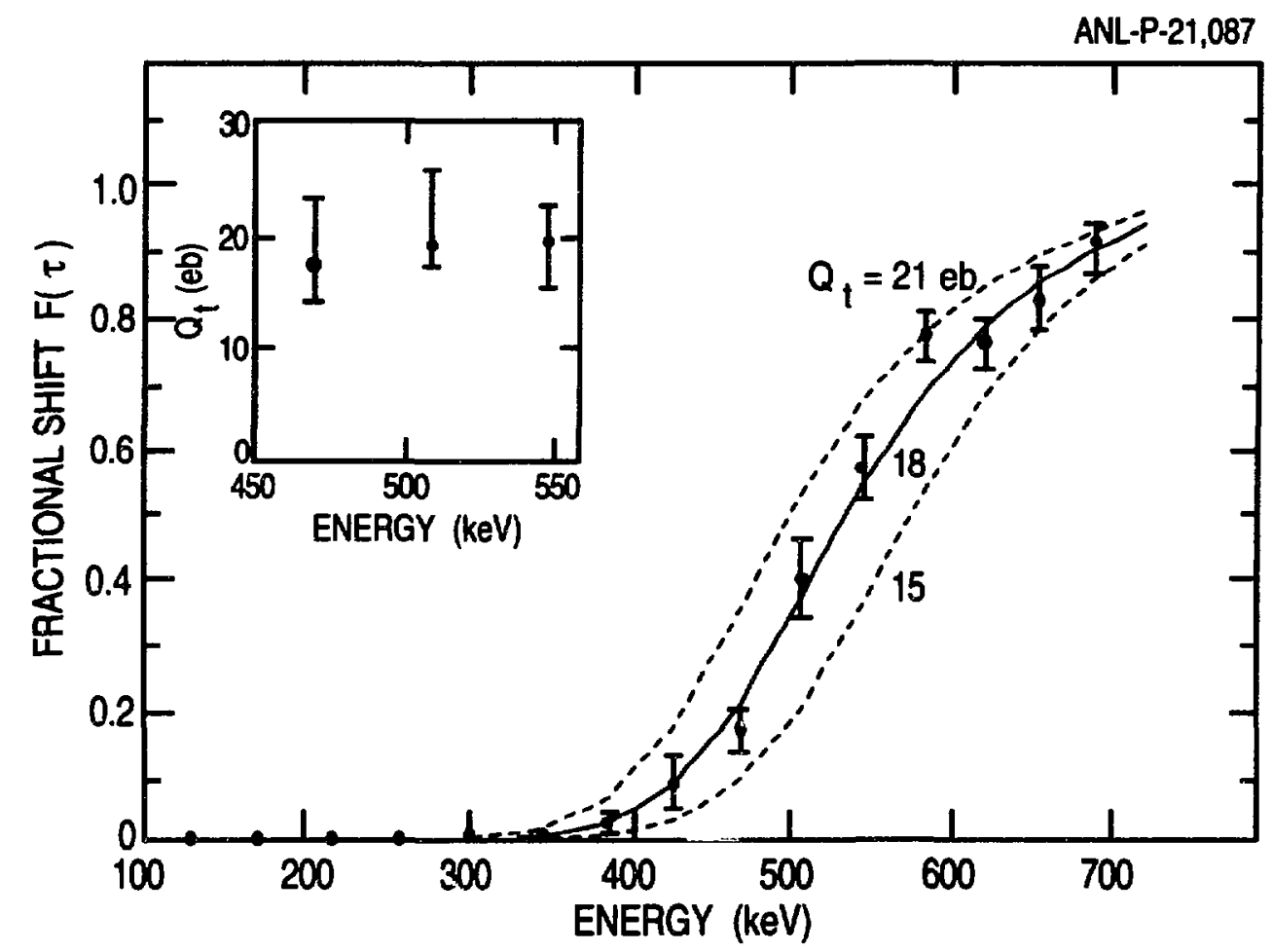

Fig. 1-18. Measured fraction of the full Doppler shift $F(\tau)$ for transitions in the superdeformed band of ${ }^{196} \mathrm{~Pb}$. The solid line corresponds to the calculated shift for a $Q_{0}$ value of $18 \mathrm{eb}$ and the dashed lines are for $Q 0$ values of 15 and $21 \mathrm{eb}$. Inset: Quadrupole moment values extracted from lineshape fits to the 468,508 , and $546 \mathrm{keV}$ transitions in the superdeformed band. 
The results can be summarized as follows. New transitions were added at the top and bottom of the previously known band. Gamma-ray directional correlations were measured for most transitions in the band verifying the expected stretched E2 character. The collectivity of the band was measured from the analysis of the Doppler shifted lineshapes of 3 SD transitions and from the fraction of full Doppler shift of $9 \mathrm{SD}$ transitions. An intrinsic quadrupole moment $\mathrm{Q}_{0}$ of $18.3 \pm$ 3.0 eb was deduced (see Fig. I-18). This value of $Q_{0}$ and those of several other SD bands in the $A \sim 190$ region were compared to the results of a number of theoretical calculations and were found to be consistent with a quadrupole deformation of $\beta_{2} \sim 0.5$. The data suggest that the deformation reaches a maximum value for $\mathrm{N}=112$ in both the $\mathrm{Hg}$ and $\mathrm{Pb} \mathrm{SD}$ bands. This trend is also present in most calculations. An analysis of the behavior of the $\mathfrak{I}^{(2)}$ moments of inertia corroborates the possibility of small differences in deformation between bands. The detailed behavior of $\mathfrak{I}^{(2)}$ as a function of rotational frequency in all $\mathrm{SD} \mathrm{Pb}$ nuclei was examined further within the framework of cranked Woods-Saxon calculations.

a.11. New Features of Superdeformed Bands in ${ }^{194} \mathrm{Hg}$ (R. V. F. Janssens, I. Ahmad, M. P. Carpenter, B. Crowell, R. G. Henry, T. L. Khoo, T. Lauritsen, B. Cederwall,*

I. Y. Lee, ${ }^{*}$ M. A. Deleplanque, ${ }^{*}$ R. M. Diamond,* P. Fallon,* A. O. Machiavelli,*

F. S. Stephens, ${ }^{*}$ M. J. Brinkmann, $\dagger$ J. A. Becker,$\dagger$ L. P. Farris, $\dagger$ E. A. Henry, $\dagger$

J. R. Hughes, $\uparrow$ M. A. Stoyer, $\dagger$ J. E. Draper,+ C. Duyar,$\ddagger$ E. Rubel,$\ddagger$ W. Satula,$\S$

I. Wiedenhoever,§ and R. Wyss§)

A striking difference between superdeformed (SD) nuclei near A $=190$ and those in the other regions is the behavior of the dynamic moment of inertia $\mathfrak{J}^{(2)}$ with the rotational frequency $\hbar \omega$. While the $\mathfrak{I}^{(2)}$ patterns of the SD bands near $A=130$ and $A=150$ show pronounced variations, the majority of the SD bands near $A=190$ display the same large, smooth increase of $\mathfrak{I}(2)$ within the frequency range $0.15<\hbar \omega<0.40 \mathrm{MeV}$. Current interpretations of this rise of $\mathfrak{I}^{(2)}$ within mean field theories invoke the gradual alignment of quasiparticles occupying high- $\mathrm{N}$ intruder orbitals in the presence of pair correlations. It is a direct consequence of these interpretations that, after the quasiparticle alignments have taken place, $\mathfrak{I}(2)$ will exhibit a downturn with increasing $\hbar \omega$ toward the rigid-body value. Up to now, no downturn in $\mathfrak{S}^{(2)}$ for the SD bands in the $\mathrm{A}=$ 190 mass region has been observed, raising some doubt as to our understanding of pair correlations and alignment effects at these large deformations.

An experiment was carried out at the 88-Inch Cyclotron facility of the Lawrence Berkeley Laboratory where excited states in ${ }^{194} \mathrm{Hg}$ were populated with the reaction ${ }^{150} \mathrm{Nd}\left({ }^{48} \mathrm{Ca}, 4 \mathrm{n}\right){ }^{194} \mathrm{Hg}$ at a beam energy of $206 \mathrm{MeV}$. The target consisted of a stack of two isotopically enriched, selfsupporting $\sim 500-\mu \mathrm{g} / \mathrm{cm}^{2} 150 \mathrm{Nd}$ foils. The gamma rays emitted in the reaction were detected with the GAMMASPHERE detector array (32 detectors for this experiment).

Compared with previous work, the three SD bands in ${ }^{194} \mathrm{Hg}$ were all extended to higher spins by two or three transitions. It is now possible to extend the dynamic moments of inertia up to frequencies larger than $0.4 \mathrm{MeV}$. In the strongest SD band, a change in the slope of $\mathfrak{I}(2)$ with $\hbar \omega$ is noticeable for $\hbar \omega \sim 0.37 \mathrm{MeV}$ and, for the first time in this mass region, a clear turnover is present around $\hbar \omega=0.43 \mathrm{MeV}$. The characteristics of the two other SD bands are very similar (see Fig. 1-19). These results can be understood, at least qualitatively, in the framework of new, improved cranked-shell-modei calculations where the monopole-pairing interaction was treated self-consistently by means of the Lipkin-Nogami method.

*Lawrence Berkeley Laboratory, †Lawrence Livermore Laboratory, $\ddagger$ University of CaliforniaDavis, §Manne Siegbahn Institute, Stockholm, Sweden 
A second important result of this work relates to the possible presence of a higher-order symmetry in SD nuclei. The appearance of a regular staggering pattern in a SD band in ${ }^{499} \mathrm{Gd}$ was presented recently as possible evidence for a new symmetry term in the Hamiltonian which is invariant under a rotation by $\pi / 2$. It was pointed out that a sequence of $4 \mathrm{p}-4 \mathrm{~h}$ states in 160 and oscillations in the $\mathrm{K}^{\pi}=0^{-}$bands in the actinide nuclei $236,238 \mathrm{U}$, might be other cases where this symmetry is realized to different degrees.

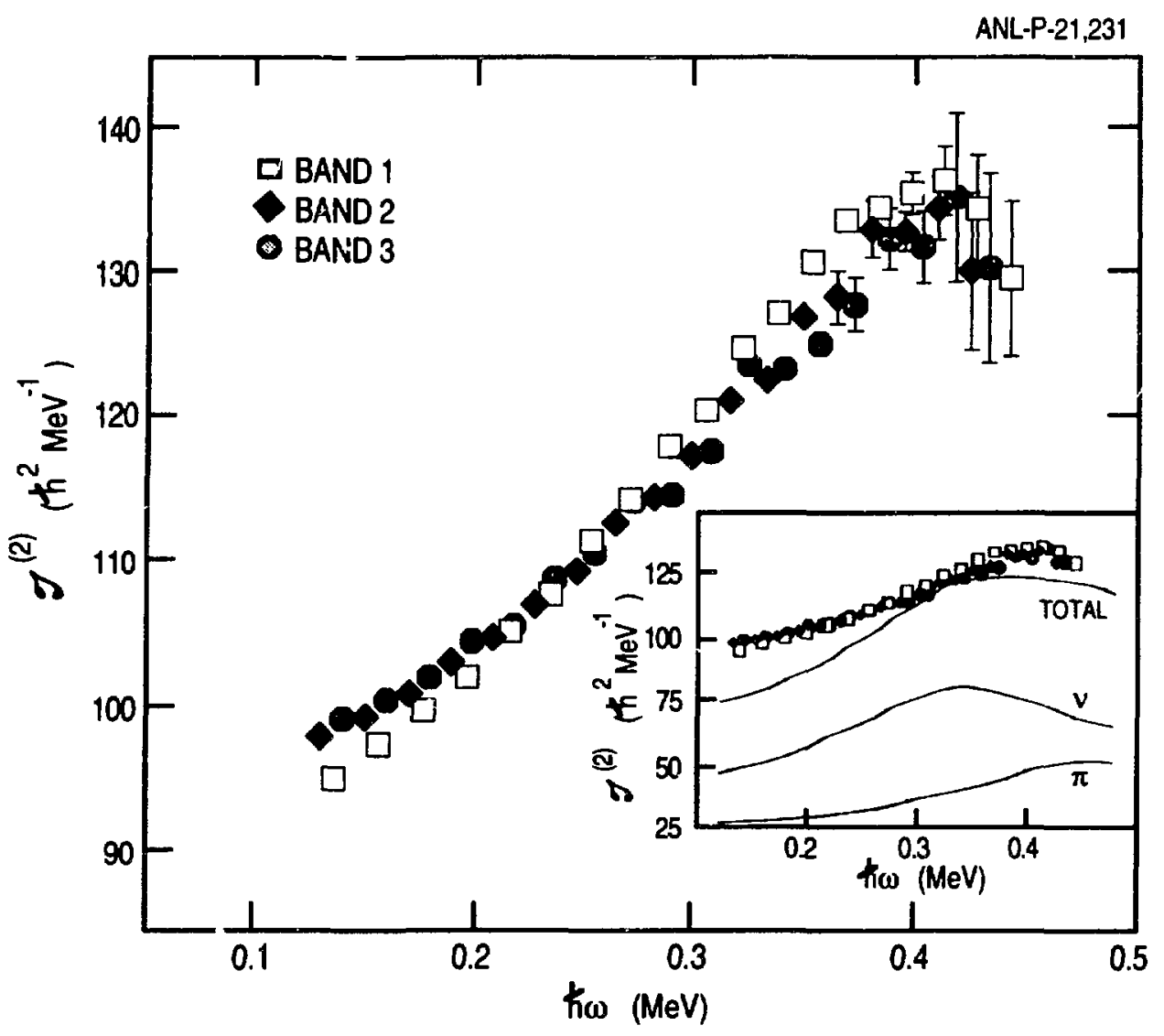

Fig. 1-19. Dynamic moments of inertia $\mathfrak{I}(2)$ as a function of $\hbar \omega$ for the SD bands in ${ }^{194} \mathrm{Hg}$. The last points for bands 2 and 3 are somewhat uncertain. The inset shows results from new, modified TRS calculations for the yrast SD configuration. The three curves in the inset represent the calculated total $\mathfrak{S}(2)$ values and their decomposition into neutron and proton contributions.

The increased resolving power obtained from high-fold coincidence data allowed us to determine the SD transition energies in ${ }^{194} \mathrm{Hg}$ with greater accuracy than in previous work and evidence for a staggering of the gamma-ray energies, similar to that observed for the SD band in ${ }^{149} \mathrm{Gd}$, is observed in all three bands (see Fig. I-20). There is at present no satisfactory detailed theoretical calculations explaining this observation. 


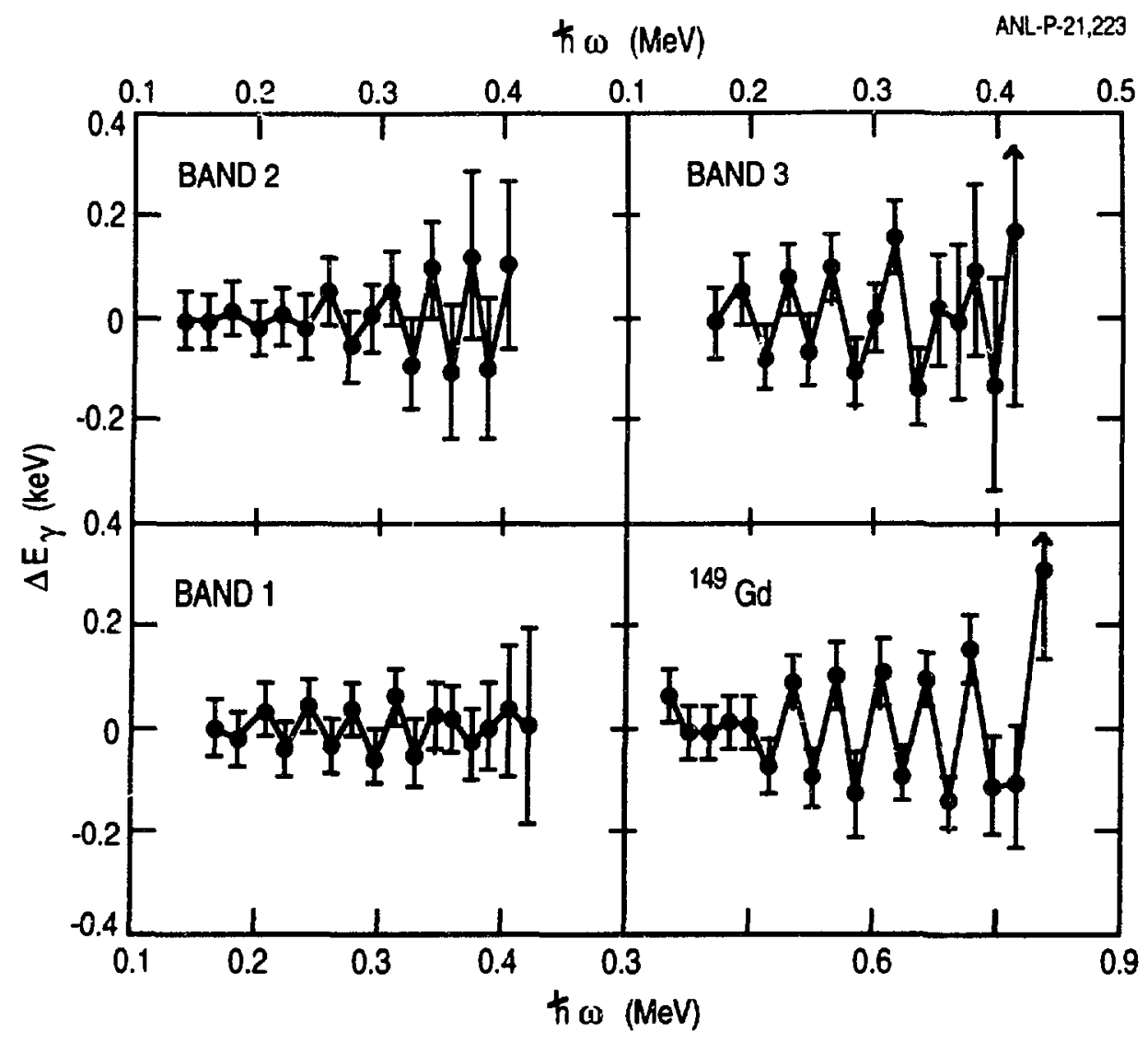

Fig. 1-20. Staggering $\Delta E$ yof the transition energies in the SD bands in ${ }^{194} \mathrm{Hg}$ and in the yrast $S D$ band in $149 \mathrm{Gd}$. The formula used to derive the staggering is :

$$
\Delta \mathrm{E}_{\gamma}(I)=\frac{3}{8}\left(E_{\gamma}(I)-\frac{1}{6}\left(4 E_{\gamma}(I-2)+4 E_{\gamma}(I+2)-E_{\gamma}(I-4)-E_{\gamma}(I+4)\right)\right.
$$

\section{a.12. Lifetime Measurement in the Superdeformed Bands of ${ }^{194} \mathrm{Hg}$}

(M. P. Carpenter, I. Ahmad, S. Harfenict, R. G. Henry, R. ' '. F. Janssens, T. L. Khoo, T. Lauritsen, J. R. Hughes, ${ }^{*}$ J. A.. Becker, ${ }^{*}$ M. J. Brinkman,* B. Cederwall, $†$

M. A. Deleplanque, $\dagger$ R. M. Diamond,$\dagger$ J. E. Draper, $\ddagger$ C. Duyar, $\neq$ P. Fallon, $\dagger$

E. A. Henry, ${ }^{*}$ R. W. Hoff,* I. Y. Lee $†$ E. Rubel, $\ddagger$ F. S. Siephens, $\uparrow$ and M. A. Stoyer*)

One of the first experiments performed by Gi: group at GAMMASPHERE was on ${ }^{194} \mathrm{Hg}$ using a thick target. The main purpose of the experiment was to look at the pioperties associated with the decay of the superdeformed band. However, due to the fact that three superdeformed (SD) bands were already identified in this nucleus, it was clear that the experimental conditions were ideal for measuring the transitional quadrupole moments $Q_{t}$ in each of the bands using the Doppler Shift Attenuaiion Method (DSAM). Our collaborators from LLNL took responsibiiity for performing the lifetime analysis.

Excited states in ${ }^{194} \mathrm{Hg}$ were populated with the ${ }^{150} \mathrm{Nd}\left({ }^{48} \mathrm{Ca}, 4 \mathrm{n}\right)$ reaction at a beam energy of 205 $\mathrm{MeV}$. The target consisted of $1.7-\mathrm{mg} / \mathrm{cm}^{2} 150 \mathrm{Nd}$ evaporated onto a $20-\mathrm{mg} / \mathrm{cm}^{2}$ Au backing. The front face of the target was covered with $200-\mu \mathrm{g} / \mathrm{cm}^{2}$ Au to retard target oxidation. Data were taken with the Early Implementation phase of the GAMMASPHERE spectrometer. For this

*Lawrence Livermore National Laboratory, †Lawrence Berkeley Laboratory, $\ddagger$ University of California, Davis. 
measurement, 25 Compton-suppressed Ge detectors were used and placed at forward and backward angles relative to the beam direction. Approximately 400 million expanded three-fold events were recorded during 3 days of beam time.

In order to perform the analysis, one dimensional spectra of backward and forward detectors were generated by placing double gates on superdeformed lines which are emitted after the recoiling nucleus has come to a stop. From these data, the fractions of full Doppler-shift $F(\tau)$ were obtained and lineshapes of individual $\gamma$ rays were analyzed to determine the dependence of the transition quadrupole moment on rotational frequency.

A previous lineshape analysis by our group at Argonne on the SD band in ${ }^{192} \mathrm{Hg}$ showed that $\mathrm{Q}_{\mathrm{t}}$ for the band was on average $20 \mathrm{eb}$ and was nearly constant with rotational frequency. There did appear to be a slight trend of decreasing $Q_{t}$ with increasing spin. For this experiment, the data were of sufficient quality to allow a DSAM analysis to be performed on two of the three SD bands. The average in-band $Q_{t}$ observed for the yrast $S D$ band is $Q_{t}=17.2(20) \mathrm{eb}$, whereas for the excited band the average is $Q_{1}=17.6(30)$ eb (see Fig. I-21). It should be noted that the measurement of the lifetimes in the excited band represent the first such lifetime information of an excited superdeformed band in this mass region. While quadrupole moments for the two bands are quite similar they are somewhat smaller than were nbtained in ${ }^{92} \mathrm{Hg}$. This is somewhat surprising especially for the excited band, since its dynamic moment of inertia is nearly identical to that of ${ }^{192} \mathrm{Hg}$. Finally, analysis of the lineshapes in both bands indicate that the side-feeding lifetimes are slower than the in-band lifetimes, an observation which was made previously for ${ }^{192} \mathrm{Hg}$.

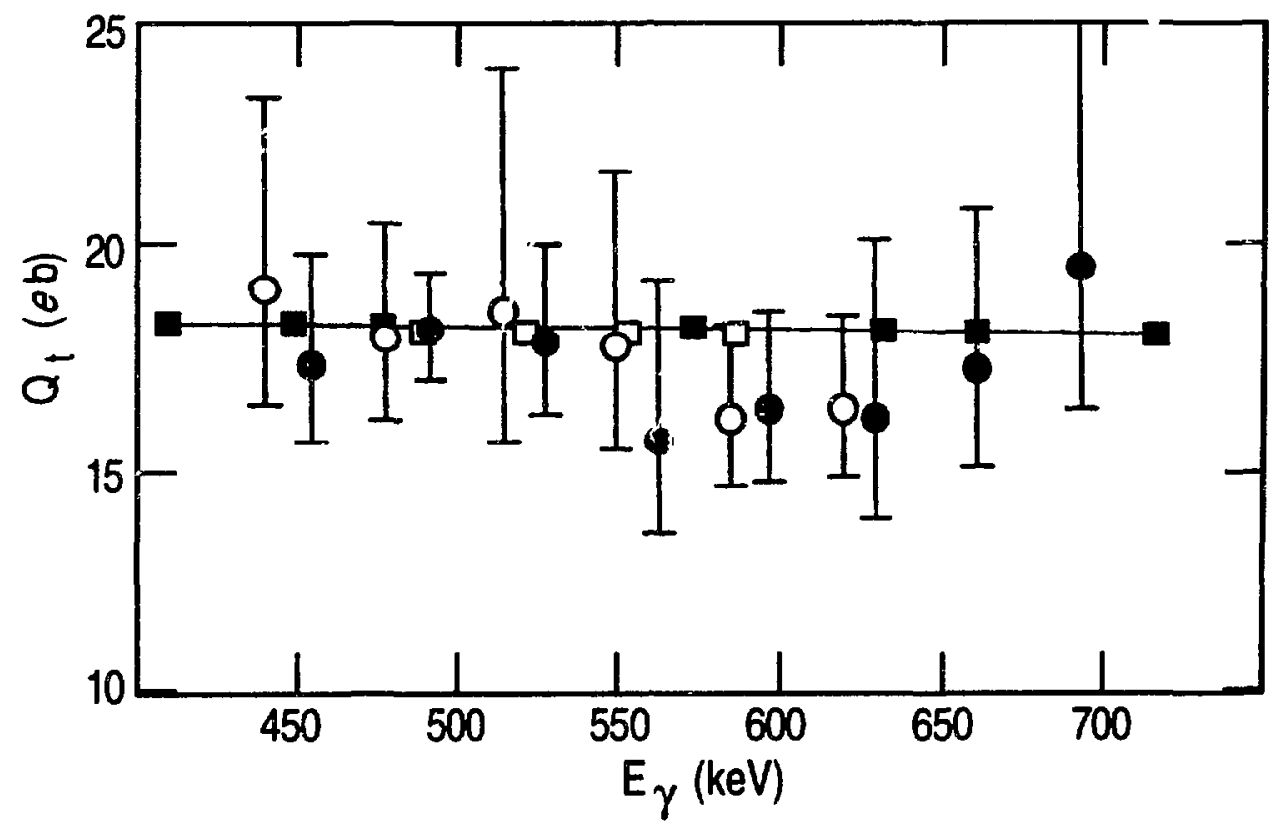

Fig. 1-21. Experimental transition quadrupole moment, $Q_{t}$, as a function of $\gamma$ ray energy for band $1(O)$ and band $2(o)$ in ${ }^{194} \mathrm{Hg}$. Theoretical values calculated with the CHF Method including pairing for band $I(\square)$, and band 2 (ए). A line joins the calculated values for band 1 . 
a.13. Search for Excited Superdeformed Bands in ${ }^{151} \mathrm{Dy}$ (D. Nisius, R.V.F. Janssens, B. Crowell, T. Lauritsen, M. P. Carpenter, I. Ahmad, R. G. Henry, T. L. Khoo, R. H. Mayer, $\dagger$ Z. W. Grabowski, $\dagger$ P. J. Daly, $\dagger$ P. Fallon, $\ddagger$ B. Cederwall, $\ddagger$ I. Y. Lee, $\ddagger$ F. S. Stephens, $\ddagger$ A. Machiavelli, $;$ M. A. Deleplanque-Stephens,$\ddagger$ R. M. Diamond, $\ddagger$ and P. J. Twin§)

Superdeformed (SD) nuclei in the mass $A=150$ region remain among the most fascinating to study because of the many unexpected features they exhibit. In particular, the observation of many "identical" SD bands in nuclei differing by several mass units remains a surprising feature for which there is currently no convincing explanation. In the first paper attempting to provide a theoretical framework to explain the first three cases in which identical SD bands have been observed (SD bands in the nuclei ( $\left.{ }^{151} \mathrm{~Tb}^{*},{ }^{152} \mathrm{Dy}\right),\left({ }^{150} \mathrm{Gd}^{*},{ }^{151} \mathrm{~Tb}\right)$ and $\left({ }^{153} \mathrm{Dy} *,{ }^{152} \mathrm{Dy}\right)-$ the * denotes an excited SD band), Nazarewicz et al. proposed an interpretation within the strong coupling limit of the particle-rotor model where the orbitals are no longer described within the Nilsson representation, but instead within the pseudo-spin coupling scheme. One of the predictions within this model is the existence of an excited SD band in ${ }^{151}$ Dy for which the transition energies are exactly midway between those of the yrast SD band in ${ }^{152} \mathrm{Dy}$. This new SD band would be the first one corresponding to a decoupling parameter $a=-1$.

The yrast SD band in ${ }^{151}$ Dy was discovered several years ago by our group in an experiment performed at ATLAS. We performed a new measurement with increased sensitivity at GAMMASPHERE, with the reaction used earlier, i.e. ${ }^{122} \mathrm{Sn}\left({ }^{34} \mathrm{~S}, 5 \mathrm{n}\right)$ at $175 \mathrm{MeV}$. Over one billion triple and higher fold coincidence events 'vere collected in a four-day run. The data analysis is still in its early stages. Nevertheless, at this point in time the following observations can be made. At least three excited SD bands were seen in ${ }^{151} \mathrm{Dy}$. One of these fits the pseudo-spin picture discussed above. The two other bands are nearly degenerate at low transition energies.

The analysis efforts continue. They focus not only on the SD structures, but also on other cascades in ${ }^{151}$ Dy.

†Purdue University, $\ddagger$ Lawrence Berkeley Laboratory, §University of Liverpool, United Kingdom

a.14. Search for Extremely Deformed Systems in ${ }^{182}$ Gs (M. P. Carpenter, R. R. Chasman, R. V. F. Janssens, I. Ahmad, B. Crowell, R. G. Henry, T. L. Khoo, T. Lauritsen, and D. Nisius)

We performed a recent experiment at GAMMASPHERE to search for elongated shapes in the nucleus ${ }^{182}$ Os. Recent calculations by $R$. R. Chasman showed that this nucleus is the most promising for finding structures with major-to-minor axis ratios of 2.2:1 or greater. These calculations include a necking degree of freedom which is thought to be an improvement over past cranked Strutinsky calculations where predictions for extended shapes in $\mathrm{Yb}$ and Er nuclei were made.

In order to populate ${ }^{182} \mathrm{Os}$ at spins $>60 \hbar$, we utilized the ${ }^{138} \mathrm{Ba}\left({ }^{48} \mathrm{Ca}, 4 \mathrm{n}\right)$ reaction. We estirnated that lighter reactions would supply enough angular momentum to the compound system but would not yield enough excitation energy to populate states with the required angular momentum in the $4 \mathrm{n}$ channel. The target was made by evaporating ${ }^{138} \mathrm{Ba}$ onto a $500-\mu \mathrm{g} / \mathrm{cm}^{2} \mathrm{Au}$ foil, followed by the evaporation of $\sim 250-\mu \mathrm{g} / \mathrm{cm}^{2}$ Au onto the exposed side of the Ba. Such a target was necessary since $\mathrm{Ba}$ is highly reactive with oxygen. Even after taking this precaution, the target suffered oxidation which adversely effected the quality of our data. 
The experiment was performed in two parts. The first part consisted of a feasibility run at ATLAS to (i) determine the optimal beam energy for the proposed experiment and (ii) test whether or not the $\mathrm{Ba}$ target would survive when exposed to the $\mathrm{Ca}$ beam. From this run, we were able to determine that a beam energy of $220 \mathrm{MeV}$ was optimal and that the GAMMASPHERE experiment was feasible even though significant oxidation of the target had taken place.

The second part of the experiment was performed at GAMMASPHERE using the predetermined energy of $220 \mathrm{MeV}$. A single target was used and the ${ }^{138} \mathrm{Ba}$ had a thickness of $750 \mu \mathrm{g} / \mathrm{cm}^{2}$. The run lasted four days and $\sim 440 \times 10^{6} 3$-fold and higher Compton-suppressed Ge events were collected. Currently, the data are being analyzed. However, no strong evidence for highly deformed structures was found.

\section{b. Shape Changes in Nuclei}

Research on the evolution of the nuclear shape as a function of spin and excitation energy along the yrast line and its vicinity concentrated mainly on nuclei near $A=190$, i.e. in the region where most of the superdeformation studies by the Argonne group are carried out. This region is of particular interest because, as one gets close to the $Z=82$ closed shell, the occupation of specific orbitals is expected to have a large effect on the overall nuclear shape. This region is also one of the very few where the cranked shell model can be tested in the limit of oblate collective rotation. In fact, in some nuclei of this region, collective bands associated with prolate and oblate collective shapes were shown to coexist. Thus, the cranked shell model can be tested in both the prolate and oblate limits in a single nucleus. Our studies have concentrated on the $\mathrm{Hg}$ isotopes with $\mathrm{A}=188-191$ which involve mainly neutron excitations and on $\mathrm{Pb}$ nuclei with $\mathrm{A}=192,195,196$ where proton excitations turn out to be particularly intriguing. Indeed, sequences of Ml transitions were observed in these nuclei. Insight into the associated quasiparticle configurations was obtained not only from the usual level properties (spins, excitation energies etc..), but also from the detailed measurement of lifetimes.

The Fragment Mass Analyzer (FMA) is now fully operational and can be used in conjunction with 10 Compton-suppressed Ge detectors for spectroscopy studies in nuclei located far from the valley of stability. The first experiments concentrated on the neutron deficient ${ }^{183} \mathrm{Hg},{ }^{186} \mathrm{~Pb}$ and $187,185 \mathrm{Tl}$ nuclei. It was shown that both proton and neutron excitations contribute to the onset of prolate deformation at low spin in these nuclei. Furthermore, the resulis on ${ }^{186} \mathrm{~Pb}$ and ${ }^{183} \mathrm{Hg}$ should also be placed in a broader context. In both nuclei some of the reported bands have transition energies "identical" to those seen in neighboring nuclei, a phenomenon first observed rather systematically in superdeformed nuclei, for which there is at the moment no satisfactory theoretical explanation. All projects at the FMA involve strong coilaborations with outside users.

Finally, other aspects of the research program reflect major efforts by collaborators from outside institutions. These include (1) the study of shape-driving orbitals in Pt-Ir-Au nuclei through lifetime measurements, (2) the study of neutron-rich nuclei from the prompt radiation of fission fragments, (3) the study of the decays of high-K isomers in ${ }^{176} \mathrm{~W}$ and (4) the study of quasiparticle excitations in neutron-rich $S n$ nuclei following complex heavy-ion-induced reactions involving the exchange of several nucleons between the target and the projectile. 
b.1. Yrast and Near-Yrast Spectroscopy in 188-190Hg (R. V. F. Janssens, M. P. Carpenter, I. Ahmad, T. L. Khoo, T. Lauritsen, I. G. Bearden, † P. J. Daly, $\dagger$ Z. W. Grabowski, $\uparrow$ B. Fornal, $\uparrow$ R. M. Mayer, $\uparrow$ U. Garg, $\ddagger$ W. Reviol,$\ddagger$ and M. W. Drigert§)

The data obtained in experiments designed to study superdeformation are usually of such quality that they allow for a very detailed study of all band structures in the nucleus under investigation. This was certainly the case for the nuclei $188,189,190 \mathrm{Hg}$ where such investigations were carried out at ATLAS over the last three years. With the ${ }^{156} \mathrm{Gd}\left({ }^{36} \mathrm{~S}, 4 \mathrm{n}\right){ }^{188} \mathrm{Hg}$ reaction at $167 \mathrm{MeV}$ and ${ }^{160} \mathrm{Gd}\left({ }^{34} \mathrm{~S}, 4 \mathrm{n}\right.$ or $\left.5 \mathrm{n}\right){ }^{190,189} \mathrm{Hg}$ reactions at 159,162 and $165 \mathrm{MeV}$, large $\gamma-\gamma$ coincidence data sets were obtained both with a stack of two $500-\mu \mathrm{g} / \mathrm{cm}^{2}$ self-supporting targets and with a 1 $\mathrm{mg} / \mathrm{cm}^{2}$ target onto which a $15-\mathrm{mg} / \mathrm{cm}^{2}$ Au or $\mathrm{Pb}$ layer was evaporated. All data $\mathrm{rts}$ were taken with the Argonne-Notre Dame $\gamma$-ray facility and contain in excess of $10^{8}$ events from which high multiplicity $\gamma$-ray cascades in the nuclei of interest can be extracted through suitable total multiplicity and sum-energy gating.

†Purdue University, $¥$ University of Notre Dame, §Idaho National Engineering Laboratory

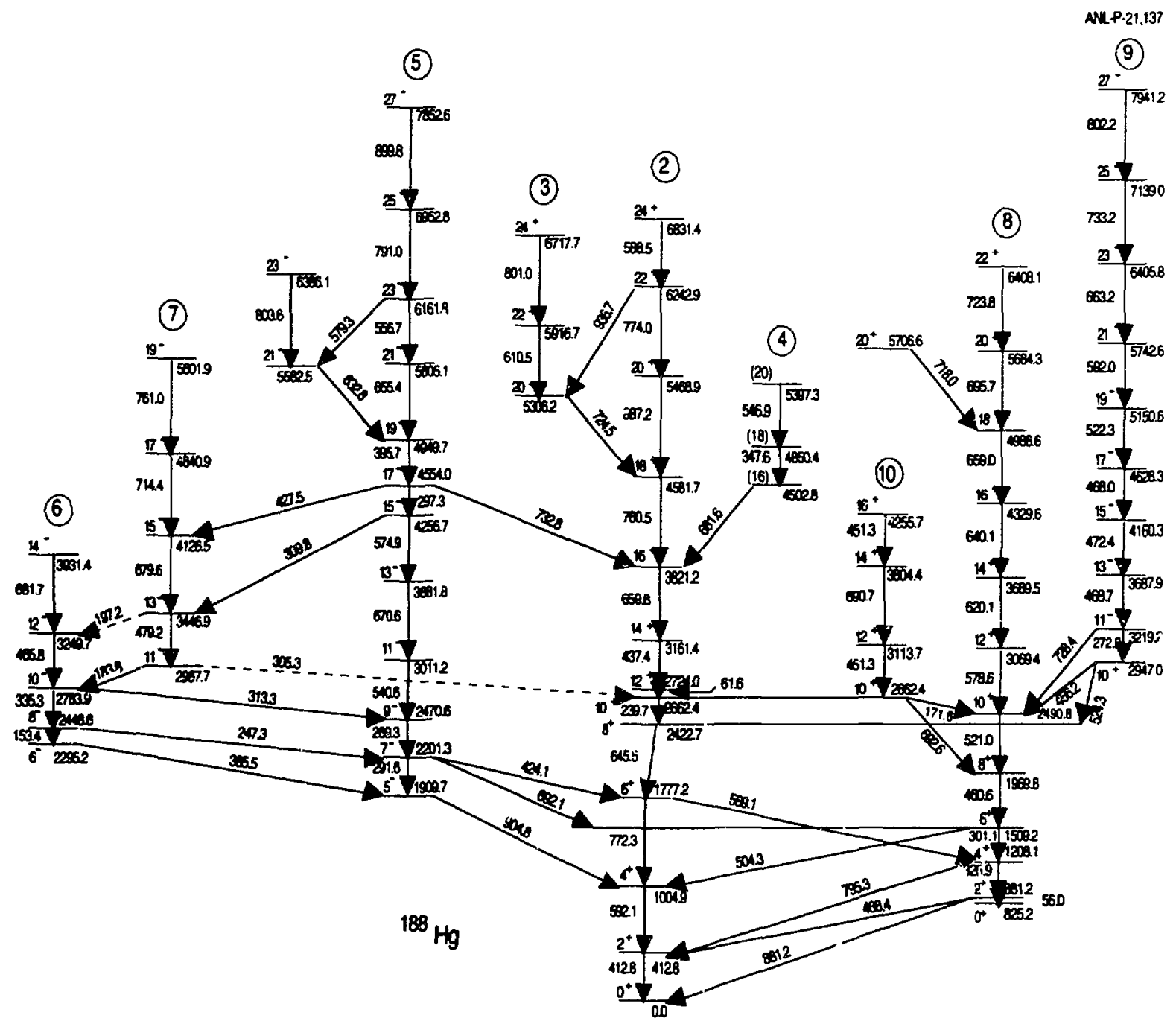

Fig. I-22. Proposed level scheme for ${ }^{188} \mathrm{Hg}$. All level energies and transition energies are given in keV. 
The experimental investigation of the level structures of all three nuclei is now complete. In ${ }^{188} \mathrm{Hg}$, two distinct types of rotational structures emerge (see Fig. I-22). One type is based on the ground state and corresponds to the rotation of an oblate, collective nucleus $\left(\beta_{2} \sim 0.15, \gamma=-60^{\circ}\right.$, in the Lund convention). A total of 7 band structures are associated with this shape (bands 1-7). From the observed energies they all appear to be of rotational character. The other 3 band structures (bands 8-10) seen in ${ }^{188} \mathrm{Hg}$ are associated with a prolate collective shape $\left(\beta_{2} \sim 0.2, \gamma=\right.$ $0^{\circ}$ ) which is known for some time to coexist with the oblate structures at low and moderate spin. In ${ }^{189} \mathrm{Hg}$ and ${ }^{190} \mathrm{Hg}$ several band structures were observed as well. In these cases it appears that the rotational structures are all associated with oblate collective rotation. However, in each nucleus other level sequences which display a more irregular pattern were also seen. It is thought that these sequences may correspond to a departure from axial symmetry. The situation appears to have some similarities with that observed in previous studies of $191,192 \mathrm{Hg}$ which were described in earlier reports.

Total Routhian surface calculations were performed for all three nuclei in order to investigate the available deformation space and determine the deformation parameters for which pronounced minima in the total energy occur. These calculations then became the starting point for detailed cranked shell-model calculations in which one tries to understand the various band crossings seen in the data and one proposes specific configurations for the observed structures.

b.2. Collectivity of Dipole Bands in ${ }^{196} \mathrm{~Pb}$ (Y. Liang, R. V. F. Janssens, M. P. Carpenter, I. Ahmad, R. G. Henry, T. L. Khoo, T. Lauritsen, E. F. Moore,* H. L. Harrington,* I. G. Bearden, P. J. Daly, $\ddagger$ B. Fornal, $\ddagger$ Z. W. Grabowski, $\ddagger$ R. H. Mayer, $\ddagger$ D. Nisius, M. Sferrazza, $\ddagger$ M. W. Drigert, $\S$ U. Garg,I and W. Reviol\$)

The study of superdeformation in ${ }^{196} \mathrm{~Pb}$ described in section B.a.10. above provided a data set of such quality that it allowed us to perform a detailed study of the yrast and near-yrast level structure in this nucleus. Of particular interest is the investigation of rotational sequences of $\mathrm{Ml}$ transitions which were observed very recently in several $\mathrm{Pb}$ isotopes at moderate spin and excitation energy. These $\Delta I=1$ bands are generally characterized by large $B(M 1) / B(E 2)$ ratios, which are characteristic of high-K proton configurations.

Last yeär we reported on the discovery of four Ml bands in $196 \mathrm{~Pb}$. Three of these are "regular", i.e. the transition energies increase smoothly with spin, suggesting a rotational behavior. The other band is "irregular", i.e. it does not display this behavior. Four bands were also found in the odd-even neighbor ${ }^{195} \mathrm{~Pb}$.

We recently completed an experiment at ATLAS in which the lifetimes of states in two of the M1 bands in ${ }^{196} \mathrm{~Pb}$ were measured with the DSAM method. The reaction ${ }^{170} \mathrm{Er}\left({ }^{30} \mathrm{Si}, 4 \mathrm{n}\right)$ was used at a beam energy of $142 \mathrm{MeV}$ and the gamma rays were detected with the Argonne-Notre Dame BGO gamma-ray facility. The recoiling nuclei were stopped in a $\mathrm{Pb}$ backing. Individual level lifetimes were extracted from the data by a detailed analysis of the Doppier-broadened gamma-ray lineshapes in the two strongest $\mathrm{Ml}$ bands. Under the assumption of pure M1 radiation, average reduced transition strengths $\mathrm{B}(\mathrm{Ml}) \sim 1.5$ W.u. were obtained in one of the "regular" bands and in the "irregular" band. In both bands the $B(\mathrm{M} 1)$ values appear to decrease with increasing spin. Cranked shell-model calculations are currently being carried out to try to account for these bands. It is likely that aligned, high-K proton configurations play an important role in these structures.

*North Carolina State University, $¥$ Purdue University, §Idaho National Engineering Laboratory, qUniversity of Notre Dame 
b.3. Collective and Quasiparticle Structures in ${ }^{192} \mathrm{~Pb}$ (M. P. Carpenter, R. V. F. Janssens, I. Ahmad, 'T. L. Khoo, T. Lauritsen, Y. Liang, A. J. M. Plompen,* M. N. Harakeh,* W. H. A. Hesseling,* G. Van't Hof,* N. Kalentar-Nayestanaki,* J. P. S. van Schagen, ${ }^{*}$ U. Garg, $†$ W. Reviol, $†$ D. Ye, $\dagger$ and I. G. Bearden)

The structure of ${ }^{192} \mathrm{~Pb}$ was investigated at ATLAS with the ${ }^{173} \mathrm{Yb}\left({ }^{24} \mathrm{Mg}, 5 \mathrm{n}\right)$ reaction at a beam energy of $132 \mathrm{MeV}$. The $0.9-\mathrm{mg} / \mathrm{cm}^{2}$-thick ${ }^{173} \mathrm{Yb}$ target was evaporated on a $15-\mathrm{mg} / \mathrm{cm}^{2}$-thick natPb backing in which the recoils were stopped. The measurement was performed with the Argonne-Notre Dame BGO $\gamma$-ray facility. The level scheme was extended up to an excitation energy of $7 \mathrm{MeV}$ and a spin of $23 \hbar$.

Two collective bands of levels linked by Ml transitions and four groups of states of non-collective character were observed. Ml bands of this type were observed in several $\mathrm{Pb}$ isotopes (see preceding contribution) and the present case is one of the few where the excitation energy of these "oblate collective" structures could be determined. Based on comparisons with experimental data on odd-A Tl isotopes and with cranked shell-model calculations assuming an oblate shape, the collective $\mathrm{Ml}$ bands are interpreted as having an underlying deformation-ailigned two-quasiproton configuration coupled to a pair of rotation-aligned $i_{13 / 2}$ quasineutrons. The four groups of noncollective level sequences are all based on isomeric states which can be understood as quasiparticle excitations within the spherical shell model. Two of these sequences are interpreted as resulting from the coupling of the $2^{+}, 4^{+}, 6^{+}$and $8^{+}$quasiparticle excitations coupled to maximally aligned two-quasineutron excitations. The two other excitations are most likely of the same character, but precise configurations are not being proposed because the available experimental information is more limited and does not allow for unambiguous assignment. Supporting evidence for this interpretation was obtained from predictions of the quasiparticle multi-step shell model of $J$. Blomqvist and collaborators. The level scheme of $192 \mathrm{~Pb}$ can be regarded as a good example of the intriguing and complex interplay between collective and single-particle degrees of freedom that occurs at moderate and high spins in nuclei near the doubly-magic ${ }^{208} \mathrm{~Pb}$ nucleus.

*Free University, Amsteráam, The Netherlands, †University of Notre Dame

b.4. Prolate Collectivity in ${ }^{187}$ Tl (M. P. Carpenter, R. V. F. Janssens, I. Ahmad, I. G. Bearden, R. G. Henry, T. L. Khoo, T. Lauritsen, Y. Liang, W. Reviol,* L. L. Riedinger,* J.-Y. Zhang,* C. R. Bingham,* W. F. Mueller,* and B. E. Zimmerman*)

A study on ${ }^{187} \mathrm{Tl}$ was carried out recently at ATLAS using the Argonne-Notre Dame BGO $\gamma$-ray Facility. The purpose of the experiment was tc study in detail the level structure of ${ }^{187} \mathrm{Tl}$ to high spin. The states in ${ }^{187} \mathrm{Tl}$ were populated with the ${ }^{156} \mathrm{Gd}\left({ }^{35} \mathrm{Cl}, 4 \mathrm{n}\right){ }^{187} \mathrm{Tl}$ reaction at beam energies of $169 \mathrm{MeV}$ and $172 \mathrm{MeV}$. The target had a thickness of $0.98 \mathrm{mg} / \mathrm{cm}^{2}$ and was backed with 16 $\mathrm{mg} / \mathrm{cm}^{2}$ of $\mathrm{Au}$ in order to stop the recoiling evaporation residues. A total of 35 million events where at least two Compton-suppressed Ge detectors fired in prompt coincidence were recorded to tape.

Two new $\gamma$ sequences of opposite parity were established from the data (see Fig. I-23). The negative-parity sequence is observed up to a spin of $43 / 2 \hbar$ and an excitation energy of $6.8 \mathrm{MeV}$ while the positive-parity sequence has been extended to $I=45 / 2 \hbar$ and an excitation energy of 5.3 $\mathrm{Mc} V$. In addition to these bands, a sequence of four $\Delta \mathrm{I}=1$ transitions accompanied by crossover transitions of stretched E2 character were also identified.

*University of Tennessee 
In order to interpret these results, it should be noted that the light mercury isotopes ( $\mathrm{N} \leq 110)$ show very nice examples of co-existing oblate and prolate structures in the same nucleus. Within this context, the strongly coupled structure identified in the data was interpreted as an oblate band built on the high-K $9 / 2^{-} \pi$ h $9 / 2$ orbital as observed in heavier odd-A Tl isotopes. The other two bands were assigned to $\pi i_{13 / 2}, K=1 / 2$ and $\pi h_{9 / 2}, K=3 / 2$ configurations, both associated with similar deformed prolate shapes of $\varepsilon_{2}-0.23$. The identification of the prolate h9/2 level structure represents the first such observation of this prolate configuration in the $\mathrm{Tl}$ isotopes.

(3)

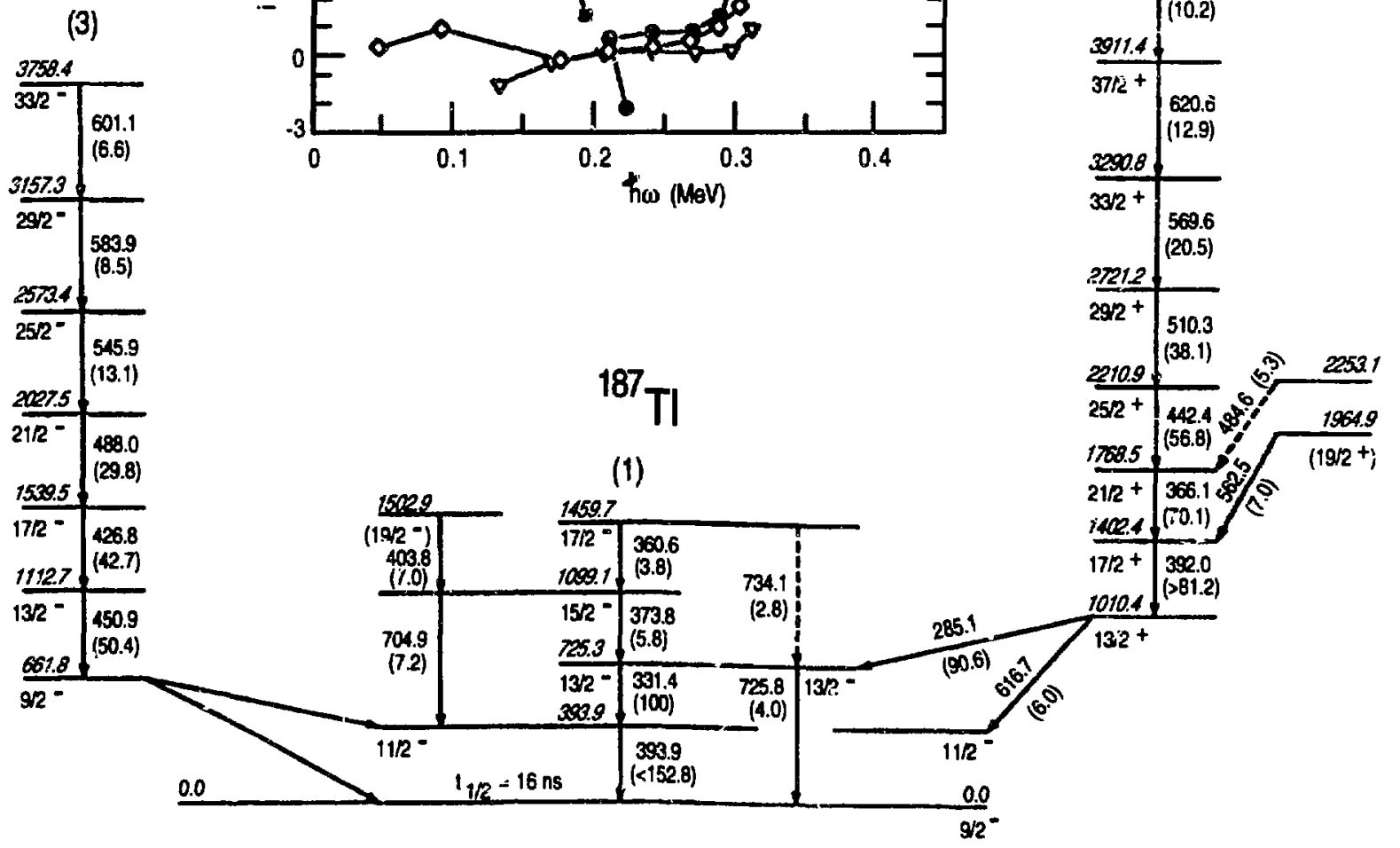

Fig. 1-23. Partial level scheme of ${ }^{187} \mathrm{Tl}$ obtained in this study. Transitions are labeled by their energy in keV; $\gamma$-ray intensities normalized to 100 for the 331.4-keV transition are given in parenthesis. Level energies are given relative to the $9 / 2-$ isomeric state. Spin-parity assignments of levels are based on DCO ratios and intensity balances. Inset: Aligned angular momentum versus rotational frequency for bands 2 and 3 in $187 \mathrm{Tl}$ and prolate bands in $186 \mathrm{Hg}$ and ${ }^{188} \mathrm{~Pb}$. The Harris parameters $\mathfrak{I}_{0}=26 \mathrm{~h}^{2} \mathrm{MeV}^{-1}$ and $\mathfrak{I}_{1}=200 \mathrm{~h}^{-1} \mathrm{MeV}^{-3}$ are used for the rotating reference. 
b.5. High-K Isumers in $176 \mathbf{W}$ (B. Crowell, D. J. Blumenthal, M. P. Carpenter, R. Henry, R. V. F. Janssens, T. L. Khoo, T. Lauritsen, Y. Liang, C. J. Lister, F. Soramel, P. Chowdhury,* S. J. Freeman, $\dagger$ and I. G. Bearden)

In ellipsoidal, a ially symmetric nuclei, the $\mathrm{K}$ quantum number is approximately conserved, where $\mathrm{K}$ is defined as the projection of the total angular momentum along the axis of symmetry. Because of the approximate conservation of $\mathrm{K}$, the decay of these high-K states is subject to selection rules, often causing them to be long-lived isomers. The high- $K$ isomers are able to decay to states of lower $\mathrm{K}$ only because of very small admixtures of the lower values of $\mathrm{K}$ in their wave-functions. Until the last decade, it was believed that the physical mechanisms of K-mixing, in the Coriolis

force, were fairly well understood, and that the size of the mixing always decreased exponentially as a function of $\Delta \mathrm{K}$, as would be expected from perturbation theory. Transitions were observed more recently in a few nuclei with large $\Delta \mathrm{K}$ and abnormally low hindrance factors, raising questions about the applicability of the Coriolis mixing picture. These transitions, however, never constituted more than a small fraction of the total decay of the high- $K$ state, with the rest proceeding through states of intermediate $K$.

In our April 1992 experiment at ANL, using the reaction ${ }^{150} \mathrm{Nd}\left({ }^{30} \mathrm{Si}, 4 \mathrm{n}\right)$, we observed an isomer in $176 \mathrm{~W}$ with a completely new type of behavior for a high-K state. The isomer, with $\mathrm{K}^{\pi}=14^{+}$ and $t_{1 / 2} \approx 70 \mathrm{~ns}$, shows an extreme!y unusual pattern of decay (Fig. I-24), with several direct

*Yale University; current address: Wellesley College, †Yale University; current address: Schuster Laboratory, Manchester, United Kingdom

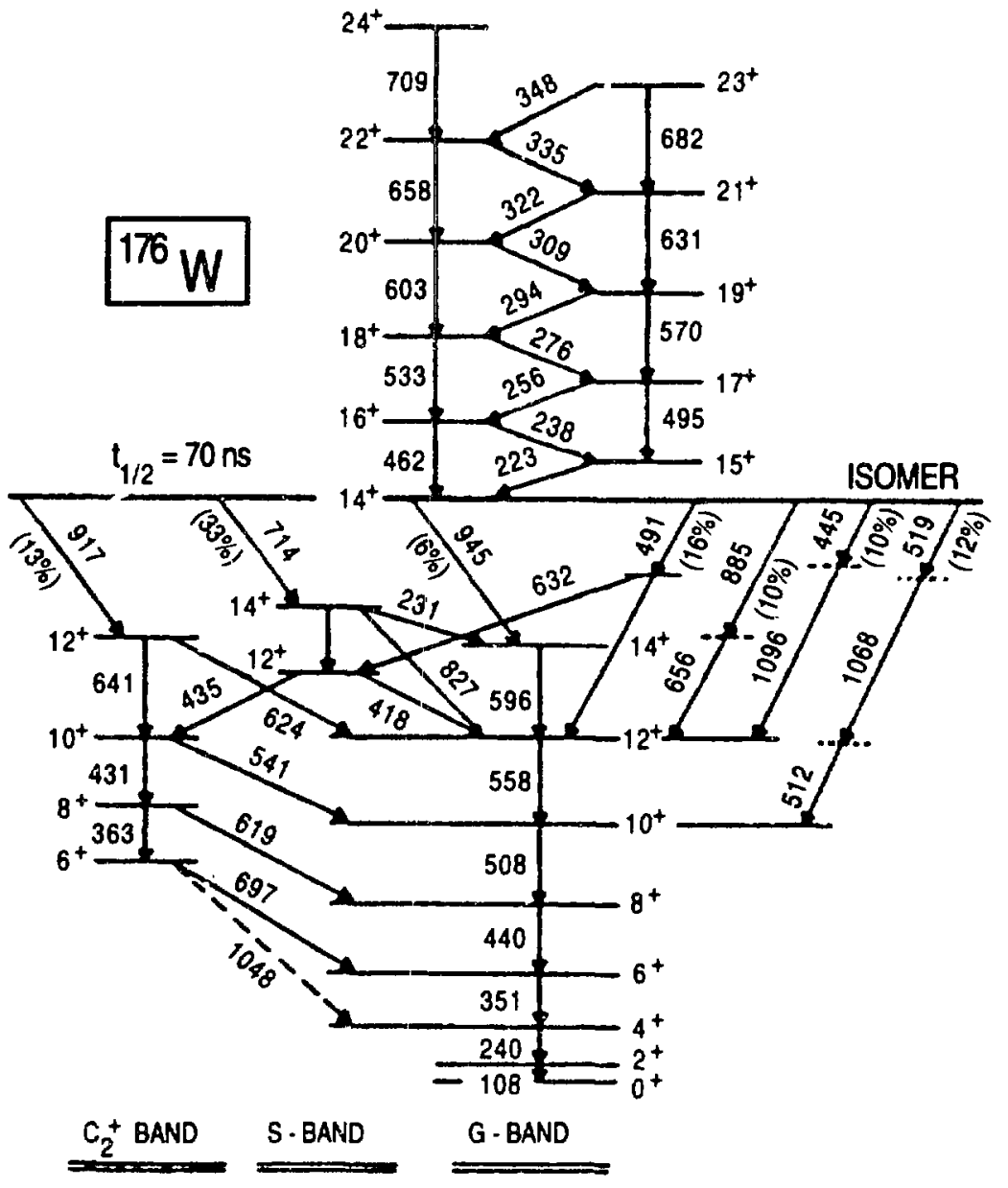

Fig. 1-24. Feeding the decay of the $K^{\pi}=14^{+}$isomer in $176 \mathrm{~W}$. 
branches to $\mathrm{K}=0$ states, and, in dramatic contrast to all previousexperience, had no detectable decay to any of the available states with $\mathrm{K}>0$ that were mapped out in our study. The isomer is only populated at the level of $2 \%$ of the $4 \mathrm{n}$ channel, and very weak gamma-ray transitions had to be detected. The combination of the availability of pulsed beams from ATLAS and detailed timin: information from the Argonne-Notre Dame BGO Array made the Argonne facility uniquely wellsuited for this type of spectroscopy.

We examined the decays of this isomer in terms of detailed theoretical calculations, with the goal of distinguishing Coriolis mixing and tunneling in the shape degrees of freedom as two possible mechanisms for $\mathrm{K}$-violation. While previous models of $\mathrm{b}^{\text {:- }}$ hly $\mathrm{K}$-violating decays using Coriolis mixing gave predictions which were not consistent with the new data, a surprisingly good explanation of these decays was achieved through models of tunneling.

In addition to the 70-ns $14^{+}$isomer, and a 10 -ns isomer at $\mathrm{J} \approx 18$, hints of the presence of a higher-spin isomer came from the following observations. An extremely weak rotational band of interlinked $\mathrm{M} 1$ and E2 transitions was observed in both prompt and delayed gamma-ray spectra. The transitions in this cascade were found to be significantly enhanced when gated on a high delayed fold in the BGO array, suggesting they were fed by a higher-lying isomer. The intensity of the strongest transition in this band was less than $1 \%$ of the $4 \mathrm{n}$ channel. The decay of the bandhead (with $\mathrm{K} \approx 13$ ) was fragmented and below the limits of detection from the statistics available from the previous experiment.

\section{b.6. Lifetime Measurements in 182Pt (I. Ahmad, I. G. Bearden, M. P. Carpenter, R. V. F. Janssens, T. L. Khoo, T. Lauritsen, J. C. Walpe, $\dagger$ U. Garg, $†$ \\ S. Naguleswaran, $\dagger$ W. Reviol, $\dagger$ J. Wei, $\dagger$ and D. Ye $\dagger$ )}

Shape coexistence at low spin has been well documented in the $\mathrm{Hg}$ isotopes. It was also proposed in the Pt isotopes on the basis of the Woods-Saxon-Strutinsky model. Strong indications of mixing between prolate and oblate shapes in the yrast bands of $184,186 \mathrm{~F}$ t were observed in lifetime measurements performed by our group and reported ear'ier. We have continued to explore this phenomenon in ${ }^{182} \mathrm{Pt}$ by measuring lifetimes of the yrast states with the Doppler-shift recoil distance technique. Lifetimes were derived for all states up to 14+. The main feature of the results is the nearly constant value of the quadrupole transition moments at low spins, in sharp contrast with the marked increase observed in $184,186 \mathrm{Pt}$. This might imply that the "mixing" between the two shapes is minimal in ${ }^{182} \mathrm{Pt}$. There also appears to be a decrease in the $\mathrm{B}(\mathrm{E} 2)$ transition probabilities after the first back-bend, which is possibly due to the onset of triaxiality.

\section{†University of Notre Dame}

b.7. Lifetime Measurements in ${ }^{181}$ Ir and ${ }^{187} \mathrm{Au}$ (R. V. F. Janssens, I. Ahmad, I. G. Bearden, M. P. Carpenter, R. G. Henry, T. L. Khoo, T. Lauritsen, J. C. Walpe, $†$ U. Garg, $\uparrow$ S. Naguleswaran, $\uparrow$ Q.-W. Ren, $\nmid W$. Reviol,$\ddagger$ L. L. Riedinger,$\ddagger$ and X.-Z. Wang $\ddagger$ )

We recently completed lifetime measurements in ${ }^{181} \mathrm{Ir}$ and ${ }^{187} \mathrm{Au}$ using the recoil distance technique. The primary aim behind these experiments was the study of the shape-driving properties of proton intruder states in the $\mathrm{A}=180$ region. The dominant bands in the ${ }^{87} \mathrm{Au}$ and 181 Ir nuclei are built on the $\pi \mathrm{h}_{9 / 2}$ and $\pi \mathrm{i}_{13 / 2}$ single particle states which are calculated to be associated with rather different prolate deformations in the most recent cranking calculations.

†University of Notre Dame, $\ddagger$ University of Tennessee 
The experiments were carried out at the ATLAS facility, using the Argonne-Notre Dame GammaRay Facility in conjunction with the Notre Dame plunger device. The reactions ${ }^{154} \mathrm{Sm}\left({ }^{31} \mathrm{P}, 4 \mathrm{n}\right){ }^{181} \mathrm{Ir}$ at $140 \mathrm{MeV}$ and ${ }^{154} \mathrm{Sm}\left({ }^{37} \mathrm{Cl}, 4 \mathrm{n}\right){ }^{187} \mathrm{Au}$ at $160 \mathrm{MeV}$ were used.

While the analysis is not completed, the following preliminary results can be mentioned. Lifetimes could be measured for the first five iransitions in the $\pi \mathrm{h} 9 / 2$ band in ${ }^{187} \mathrm{Au}$ as well as for the $21 / 2^{+}$. $17 / 2+$ transition in the $\pi \mathrm{i} 13 / 2$ band. It is clear from the analysis that the $\pi \mathrm{i} 13 / 2$ orbital does correspond to a larger deformation $\left(\mathrm{Q}_{t}=8.2 \pm 0.7 \mathrm{e.b}\right)$ than the $\pi \mathrm{h} 9 / 2$ orbital $\left(\mathrm{Q}_{t}=6.2 \pm 0.7 \mathrm{e.b}\right)$. The same observation appears to be valid for the two orbitais in ${ }^{181} \mathrm{Ir}$, where the average $\mathrm{Q}_{\mathrm{t}}$ value $(6.1 \pm 0.7 \mathrm{eb})$ in the $\pi \mathrm{h} 9 / 2$ band is again lower than the similar quantity $(8.9 \pm 0.8 \mathrm{eb})$ in the $\pi \mathrm{i}_{13 / 2}$ band. It is also clear from the data that the $\pi \mathrm{i}_{13 / 2}$ band has larger deformation in ${ }^{181}$ Ir than in ${ }^{187} \mathrm{Au}$, in keeping with the prediction in cranked shell model calculations that this should happen because the proton Fermi level has now risen closer to the $\pi$ i $13 / 2$ orbital.

b.8. High-Spin Gamma-Ray Spectroscopy of ${ }^{96-98}$ Ru (M. P. Carpenter, I. Ahmad, R. V. F. Janssens, T. L. Khoo, T. Lauritsen, Y. Liang, W. Reviol,* U. Garg,* A. Aprahamian,* B. Davis,* S. Naguleswaran,* J. C. Walpe,* D. Ye,* and I. G. Bearden)

It was predicted by $\AA$ berg that nuclei with mass $90-100$ (e.g. ${ }^{96} \mathrm{Ru}$ ) exhibit collective rotation associated with a "dumb-bell" shape. In order to test this prediction, an experiment was performed at ATLAS which populated excited states in the nuclei ${ }^{96,97,98} \mathrm{Ru}$ using the ${ }^{65} \mathrm{Cu}\left({ }^{36} \mathrm{~S}\right.$,pxn) reaction at 135 and $142 \mathrm{MeV}$. The $\gamma$ decay of these nuclei was measured with the Argonne-Notre Dame BGO facility. The ${ }^{65} \mathrm{Cu}$ target had a thickness of $0.5 \mathrm{mg} / \mathrm{cm}^{2}$ and was evaporated onto a Au foil in order to stop all recoils at the target position.

We established from the coincidence data gated on BGO multiplicities $K \geq 8$ and $K \geq 15$ two main band structures in $96,97,98 \mathrm{Ru}$, and thus extended significantly the previously-known high-spin level structure of all three nuclei. The $\gamma$ transitions observed in one of these two structures suggest that this sequence is rotational in character and co-exists with the near-spherical ground state, i.e. the transitions connecting levels in these sequences are stretched quadrupoles and show a smooth increase in energy with increasing spin. Recent Total Routhians Surface (TRS) calculations predict the exis.ence of deformed prolate neutron configurations with negative parity in all three Ru nuclei at near yrast energies. The configurations associated with these minima involve the $\mathrm{vh}_{11 / 2}$ orbital and the deform-ations are quite small $\left(\beta_{2}<0.13\right)$. According to these calculations, an addition of a pair of aligned $\mathrm{g}_{9 / 2}$ protons is important in stabilizing these minima. For ${ }^{97} \mathrm{Ru}$, the favored configuration for the collective band is $\left[\mathrm{vh}_{11 / 2} \otimes\left(\pi \mathrm{g}_{9 / 2}\right)^{2}\right]$. For the even-A nuclei, the neutron configuration associated with these minima is $\left[\mathrm{vh}_{11 / 2} \otimes \pi \mathrm{g} 7 / 2\right]$. The data also contain evidence for a weak sequence of $\gamma$ rays with very regular energy spacings $(\Delta E \gamma \cong 60 \mathrm{keV})$. In order to gain more infornation on this structure, which may be suggestive of large deformation, an experiment was compieted very recently at GAMMASPHERE. The analysis is in progress.

*University of Notre Dame 
b.9. The Level Structure of the Odd-Odd Nucleus ${ }^{224}$ Ac (I. Ahmad, J. E. Gindler,* M. P. Carpenter, D. J. Henderson, E. F. Moore,† R. V. F. Janssens, I. G. Bearden, and C. C. Foster $\$$ )

The level structure of the odd-odd nucleus ${ }^{224} \mathrm{Ac}$, which is expected to contain large octupoleoctupole correlations, was studied by measuring alpha, gamma, and electron spectra associated with the decay of the parent ${ }^{228} \mathrm{~Pa}\left(\mathrm{t}_{1 / 2}=22 \mathrm{~h}\right)$. The ${ }^{228} \mathrm{~Pa}$ activity was produced by the irradiation of ${ }^{232} \mathrm{Th}$ foils with $45-\mathrm{MeV}$ protons at the Indiana University Cyclotron Facility. The irradiated foil was brought to Argonne, where Pa was chemically separated from the bulk ${ }^{232} \mathrm{Th}$ and fission products. Thin sources of the purified $\mathrm{Pa}$ fraction were prepared and these were used for the measuremerits of alpha singles as well as alpha-gamma, alpha-gamma-gamma and alpha-electron spectra. From a detailed analysis of the data, a partial level scheme for ${ }^{224} \mathrm{Ac}$ was constructed (see Fig. I-25, I-26).

*Chemistry Division, ANL, $\uparrow$ North Carolina State University, §Indiana University Cyclotron Facility

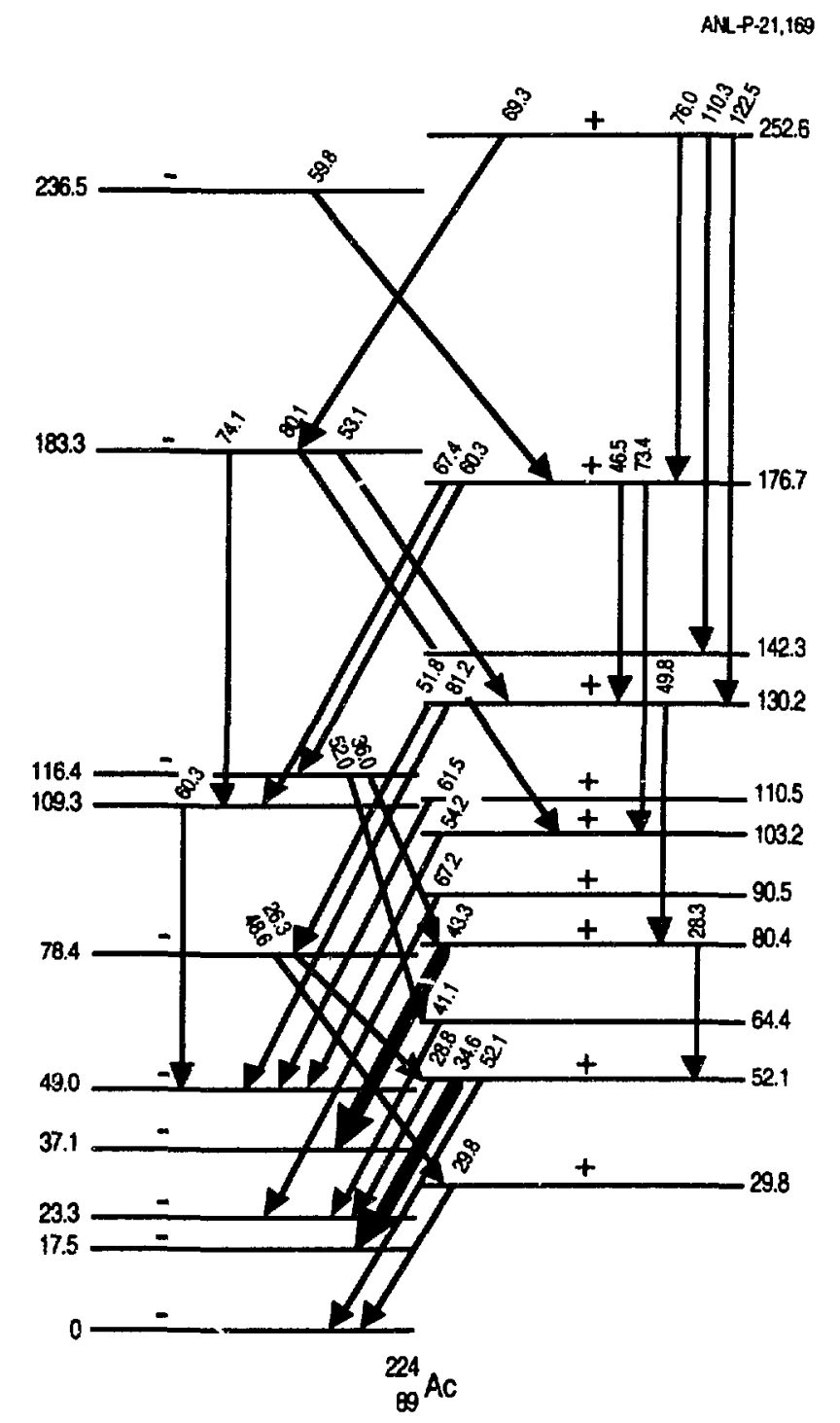

Fig. I-25. Low-energy portion of the 224 Ac level scheme deduced in the present study. Positiveparity levels are shown on the right side and the negative parity on the left. 
Although spins and parities of most of the levels were not uniquely determined, the data show the presence of positive and negative parity bands and fast $E l$ transitions between them. Two bands at 354 and $360 \mathrm{keV}$, which are shown to have opposite parities, appear to be the members of a parity doublet. The hindrance factors of alpha transitions to these levels are very similar suggesting similarities in the wavefunctions of the two states. The similarity between the wavefunctions of the members of the parity doublet constitute the strongest evidence of octupole deformation.

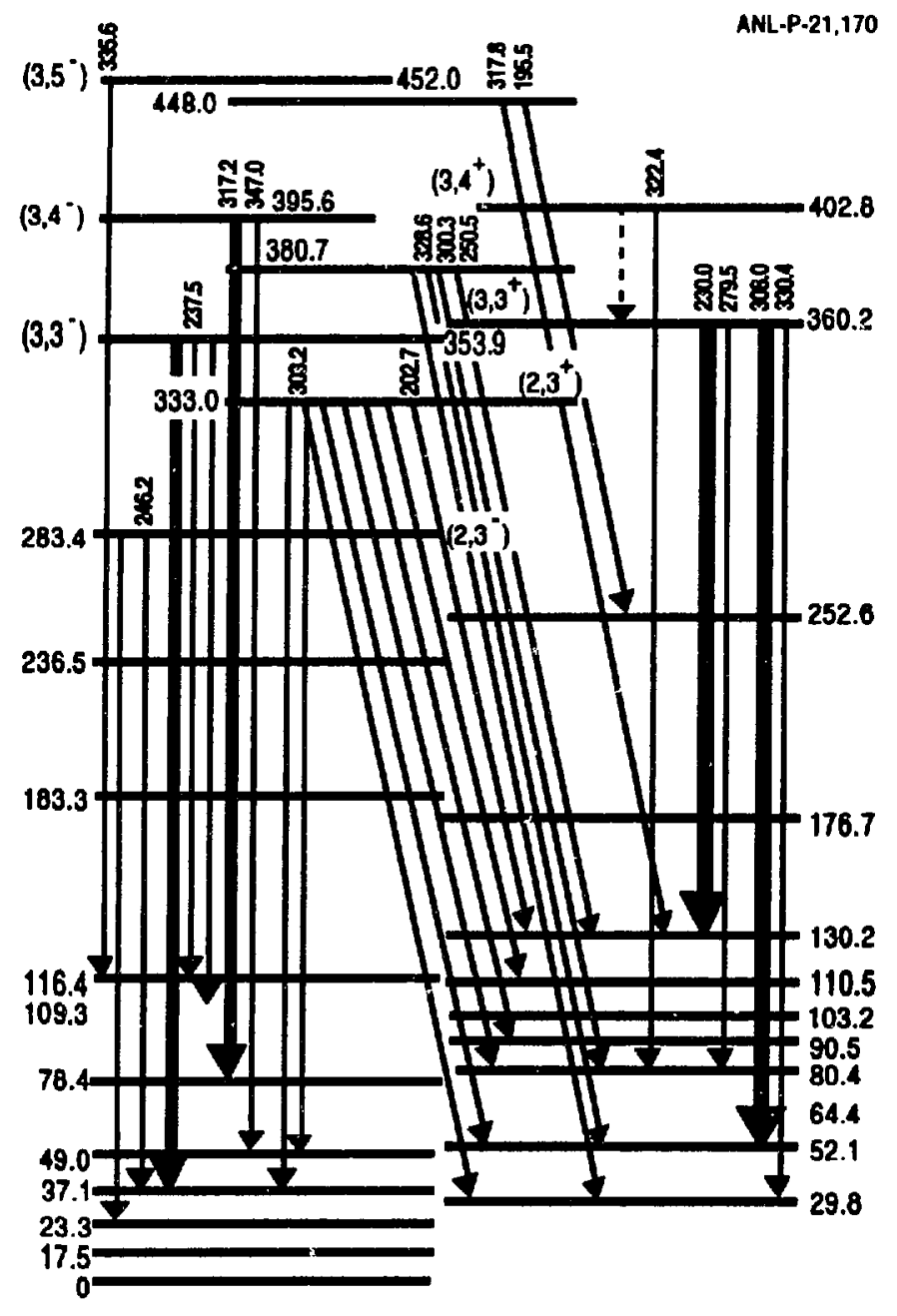

Fig. I-26. Higher energy portion of the level scheme of ${ }^{224}$ Ac deduced in the present work. Positive-parity levels are shown on the right side and the negative parity states on the left.

\section{b.10. Octupole Shapes in Nuclei (I. Ahmad and P. A. Butler*)}

A review article on "Octupole Deformation in Nuclei" was published. The article summarizes the current experimental knowledge about the octupole shapes in nuclei. The review shows that some odd-mass nuclei in the Th region contain large octupole-octupole correlations in their ground states. In particular, the odd-proton $\mathrm{Ac}$ and $\mathrm{Pa}$ nuclei exhibit the maximum correlations. At medium spins, nuclei in the mass 224 as well as mass 144 regions exhibit properties characteristic of reflection asymmetric shape. The article concludes that at present many nuclei were observed with soft octupole shape but no example was found for the permanent octupole deformation.

*University of Liverpool, United Kingdom 


\section{b.11. Lifetime Measurements in New Neutron-Rich Nd Isotopes with the Doppler Profile Method (I. Ahmad, C. J. Lister, L. R. Morss, ${ }^{*}$ K. L. Nash,* C. W. Williams, ${ }^{*}$ W. R. Phillips, $\dagger$ J. L. Durell, $\nmid$ F. Liden, $\dagger$ C. J. Pearson, $\dagger$ J. A. Shannon, $\dagger$ B. J. Varley, $\dagger$ N. Schulz, $\ddagger$ M. Bentaleb, $\ddagger$ and E. Lubkiewicz $\ddagger)$}

Coincidences between prompt gamma rays emitted in the decay of fission fragments from a ${ }^{248} \mathrm{Cm}$ source were studied using the EUROGAM array of 45 suppressed Ge-detectors augmented by 5 LEPS detectrrs. A $5-\mathrm{mg}{ }^{248} \mathrm{Cm}$ source, mixed with $65-\mathrm{mg} \mathrm{KCl}$ and pressed in the form of a 7$\mathrm{mm}$ diameter pellet was used for this experiment. Transitions in the isotopes ${ }^{154} \mathrm{Nd}$ and ${ }^{156} \mathrm{Nd}$, about which nothing was previously known, were identified by gating on gamma rays in the complementary $\mathrm{Kr}$ fragments. From this analysis, yrast levels in the ground-state bands of ${ }^{152} \mathrm{Nd}$, ${ }^{154} \mathrm{Nd}$ and ${ }^{156} \mathrm{Nd}$ were identified up to spin $16 \hbar$. The static moments of inertia for the groundstate bands of these isotopes were found to be $41.2,42.2$ and $44.6 \hbar^{2} \mathrm{MeV}^{-1}$. The moments of inertia for each band increase slowly with spin up to an expected band crossing near $\hbar \omega=0.3$ $\mathrm{MeV}$, indicating a strong interaction between the ground and $\mathrm{s}$ bands in each nucleus. It is assumed that the ground and $s$ bands have similar deformations in each nucleus separately and that measurements of the lifetimes of the high-spin states will give a measure of the intrinsic (charge) quadrupole moment of each ground state. Clean, gated spectra for transitions in each isotope were obtained from triple-gamma coincidence data. These spectra showed symmetrically broadened Doppler profiles for transitions from the $12^{+}, 14^{+}$and $16^{+}$levels, which have lifetimes of $\sim 1$ to $\sim 2$ ps, comparable to the slowing-down times of the $\mathrm{Nd}$ fragments in the $\mathrm{KCl}$ source pellet. Fitting these Doppler profiles using a simple model for the feeding of the states and assuming a constant intrinsic quadrupole moment for each nucleus separately gave preliminary values for the quadrupole moments of $7.5(+2.3,-0.7), 7.6(+0.6,-0.5)$ and $7.6(+0.7,-0.6)$ eb for isotopes with $\mathrm{N}=92,94$ and 96 , respectively. The errors on these moments do not include any systematic error from lack of precise knowledge of the stopping power. However, the relative values have no uncertainty from that source. The preliminary results suggest that the charge deformation in these isotopes changes only slightly as the isospin is increased, once large deformation has already been established.

*Chemistry Division, ANL, †University of Manchester, United Kingdom, †CRN, Strasbourg, France

\section{b.12. The Observation of a Two-Quasi-Neutron Rotational Band in ${ }^{102} \mathrm{Zr}$ (I. Ahmad, C. J. Lister, L. R. Morss, ${ }^{*}$ K. L. Nash, ${ }^{*}$ C. W. Williams, ${ }^{*}$ W. R. Phillips, $\dagger$ J. L. Durell $\uparrow$ F. Liden, $\dagger$ C. J. Pearson, $\dagger$ J. A. Shannon, $\dagger$ B. J. Varley, $\dagger$ N. Schulz, $\ddagger$ M. Bentaleb, $\ddagger$ and E. Lubkiewicz $\ddagger$ )}

Triple-gamma coincidence data on prompt gamma-rays emitted from ${ }^{248} \mathrm{Cm}$ fission fragments were obtained using the EUROGAM gamma-detector array. Analysis of data on $102 \mathrm{Zr}$ led to the observation of a two-quasi-particle rotational band. The properties of this band confirm assignments made in earlier work and give information on pairing in neutron-rich nuclei near $\mathrm{A}=$ 100. The bandhead lies at an energy of $1821 \mathrm{keV}$ and the band shows near-perfect rotational characteristics. From the level spacings the $\mathrm{K}$ quantum number of the band was unambiguously determined to be 4 . Our previous work established the ground-state configurations of $101 \mathrm{Zr}$ and ${ }^{103} \mathrm{Zr}$ as the $3 / 2^{+}[411]$ and $5 / 2-[532]$ Nilson single-particle orbitals, respectively. An excited band with $\mathrm{I} \pi=5 / 2^{-}$was also observed in ${ }^{101} \mathrm{Zr}$. It is therefore likely that the two-quasi-particle band observed now in ${ }^{102} \mathrm{Zr}$ has the intrinsic structure of the parallel coupling of the $3 / 2^{+}$and $5 / 2^{-}$ neutron states, i.e. $I^{\pi}=4$. Confirmation of this assignment comes from the gamma-ray branching ratios which determine the ratio $\left(\mathrm{g}_{\mathrm{K}}-\mathrm{g}_{\mathrm{R}}\right) / \mathrm{Q}_{0}$ for a rotational band, where $\mathrm{g}_{\mathrm{K}}$ is the gyromagnetic

*Chemistry Division, ANL, †University of Manchester, United Kingdom, ‡CRN, Strasbourg, France 
ratio for the intrinsic state, $\mathrm{g}_{\mathrm{R}}$ is that for the rotational motion and $\mathrm{Q}_{0}$ is the intrinsic quadrupole moment. The measured values for the two quasi-neutron band are in excellent agreement with those expected for the proposed structure as calculated from the observed ratios for the singleparticle bands in ${ }^{101} \mathrm{Zr}$ and ${ }^{103} \mathrm{Zr}$. Further data analysis is in progress to search for and identify other excited rotational bands in neutron-rich nuclei in the mass 100 region. These data will not only provide information on the single-particle configurations in this region, but also lead to estimates of the pairing gap far from stability.

\section{b.13. Gamma Spectrum Following Neutron Capture in ${ }^{167} \mathrm{Er}$ (D. Visser*, \\ T. L. Khoo, R. G. Henry, T. Lauritsen, I. Ahmad, M. P. Carpenter, B. Crowell, \\ R. V. F. Janssens, C. J. Lister, D. Nisius, J. L. Durell, $\uparrow$ W. R. Phillips, $\uparrow$ \\ J. A. Shannon, $\uparrow$ B. J. Varley, $\dagger$ R. Casten, $\ddagger$ and R. Gill $\ddagger)$}

We measured the spectrum of gamma rays that are emitted after capture of neutrons in ${ }^{167} \mathrm{Er}$, using Compton-suppressed Ge detectors from TESSA at the BNL reactor. The measuremant was motivated by our experiment on the spectrum of $\gamma$ rays from the decay out of SD bands (B.a.l.). In both cases, $\gamma$ decay originates from a sharp highly excited state, and we wanted to compare the spectra. The ${ }^{168} \mathrm{Er}$ spectrum will also provide information on the $\gamma$ strength function and on level densities. The spectrum, which was corrected for detector response and efficiency, reveals primary (first-step, high-energy) transitions up to nearly $8 \mathrm{MeV}$, secondary (last-step, lowerenergy) transitions, as well as a continuous statistical component. The spectrum from decay out of the SD band in ${ }^{192} \mathrm{Hg}$ also shows these features. Monte Carlo calculations of the decay process will be undertaken to generate the $\gamma$ spectrum. The sensitivity of the spectrum to the $\gamma$ strength function and to level densities will be explored.

*Western Michigan University, †University of Manchester, United Kingdom, $\ddagger$ Brookhaven National Laboratory

\section{b. 14. Gamma-Ray Studies of Neutron-Rich Products of Deep-Inelastic Heavy-Ion Reactions (M. P. Carpenter, R. G. Henry, R. V. F. Janssens, T. L. Khoo, T. Lauritsen, Y. Liang, F. Soramel, 1. G. Bearden, B. Fornal $\nmid \uparrow$ R. H. Mayer, $\ddagger$ D. Nisius, $\ddagger$ \\ M. Sferrezza, $\ddagger$ R. Broda, $\nmid$ P. J. Daly, $\ddagger$ and Z. W. Grabowski, $\ddagger)$}

By thick target $\gamma$ coincidence measurements, we are exploring the spectroscopy of neutronexcessive products of heavy-ion collisions that cannot be reached by fusion-evaporation reactions. Since the production method unavoidably delivers many product nuclei with similar yields, the data analysis is often formidable, but it can take full advantige of the sensitivity and selectivity of modern $\gamma$-ray detector arrays.

Our first application of these techniques was to neutron-rich sdf-shell nuclei produced in reactions of ${ }^{34} \mathrm{~S},{ }^{36} \mathrm{~S}$ and ${ }^{37} \mathrm{Cl}$ beams on ${ }^{160} \mathrm{Gd}$ targets just above the Coulomb barrier. High quality $\gamma /$ data came to us as byproducts of ATLAS studies of superdeformed bands in the A $=190$ region, and information about deep inelastic products could be extracted from lower multiplicity subsets of the $\gamma$ events. Notable results included the observation of yrast cascades in four $\mathrm{N}=20$ isotories, and of previously unknown yrast states in ${ }^{33} \mathrm{Si},{ }^{34} \mathrm{P},{ }^{38} \mathrm{~S}$, and ${ }^{39} \mathrm{Cl}$.

Subsequently, dedicated $\gamma \gamma$ experiments were performed at ATLAS for the systems ${ }^{122,124} \mathrm{Sn}+$ $325 \mathrm{MeV}{ }^{76} \mathrm{Ge},{ }^{122,124} \mathrm{Sr}+344 \mathrm{MeV}{ }^{80} \mathrm{Se}$ and ${ }^{124} \mathrm{Sn}+665 \mathrm{MeV}{ }^{136} \mathrm{Xe}$. The main aim was to investigatc the yrast spectroscopy of heavy tin nuclei not accessible by fusion-evaporation. The data obtained included new information about yrast states of moderately high spins in more than 50 neutron-rich nuclei. Isotopic assignments for previously unknown $\gamma$-ray cascades rest in part on

†INP, Cracow, Poland, $\ddagger$ Purdue University 
observed $\gamma \gamma$ cross-coincidences between light and heavy partner products. Results for tin isotopes in the mass-range $A=119-126$ feature decay schemes for ten new yrast isomers and include a complete series of $B(E 2)$ determinations for $\left(v_{11 / 2}\right)^{n}$ states $(n=2,3)$. The tiny $B\left(E 2,27 / 2^{-}-23 /\right)^{-}$ ) observed in ${ }^{123} \mathrm{Sn}$ - less than 0.002 W.u. - is a clear manifestation of half-filling of the $v_{11 / 2}$ subshell near $\mathbf{N}=73$. Yrast cascades in several neutron-rich $\mathrm{Sb}$ and In nuclei were also identified.

Extensive yield distributions with $\mathrm{A}$ and $\mathrm{Z}$ for projectile-like and target-like even-even products of the above reactions were obtained from measured $\left(4^{+}-2^{+}, 2^{+}-0^{+}\right) \gamma$ coincidence intensities. Analysis of these distributions gave estimates of the average number of evaporated neutrons, which were then used to extract yield patterns for primary products. Calculations of minimum potential energy surfaces for the appropriate dinuclear systems can explain satisfactorily the gross features of the observed yield patterns. Moreover, they enable one to make reliable predictions on the basis of $\mathrm{N} / \mathrm{Z}$ equilibration concerning the outcome of future experiments using other targetprojectile combinations.

\section{b.15. Structure Studies in the Light $\mathrm{Hg}$ Isotopes from Gamma-FMA Coincidence Measurements (R. G. Henry, R. V. F. Janssens, M. P. Carpenter, C. Davids, T. L. Khoo, T. Lauritsen, F. Soramel, Y. Liang, I. G. Bearden, B. Fornal, $\dagger$ R. M. Mayer, $†$ D. Nisius $\nmid$ M. W. Drigert, $\ddagger$ W. Reviol,$\S$ W. Chung, $\S$ and K. BindraI)}

We completed the first experiment at the FMA where the device was used in conjunction with 10 Compton-suppressed Ge detectors located at the target position. The main purpose of the experiment' was to evaluate the capabilities of the setup in a case where (1) several reaction channels of widely different yields are present, (2) where weak gamma-ray branches are present (to study the limits of detection sensitivity), (3) where isomers occur (which affect the FMA transmission and allow the investigation of the use of thin $C$ foils to reset the charge-state dis,tribution of the recoils), and (4) where some new interesting physics questions may be addressed.

In the previcus year $v e$ reported on the results of the transmission and charge-state dependence on isomers, internal conversion, the thickness and distance of $C$ foils from the target, as well as the optimum gating parameters and conditions for sorting gamma-ray spectra. The analysis of gammarecoil and gamma-gamma-recoil data demonstrates the power and sensitivity of the device. The superdeformed band in ${ }^{192} \mathrm{Hg}$ which is $2 \%$ of the $192 \mathrm{Hg}$ yield and $0.5 \%$ of the total reaction products was easily detected in gamma-gamma-recoil coincidences. This was the first direct identification of a superdeformed band with the recoils from its nucleus.

The gamma spectra in coincidence with all $\mathrm{Hg}$ masses from 189 to 193 were extracted and the gamma rays identified from previous studies indicated that these were indeed very clean spectra. The weakest residue analyzed was ${ }^{189} \mathrm{Hg}$ which arose from the reaction on an impurity Gd isotope in tive ${ }^{160} \mathrm{Gd}$ target. The ${ }^{189} \mathrm{Hg}$ recoils comprised only $0.7 \%$ of the total reaction products and still a very clean gamma-ray spectrum was extracted. A gamma-gamma-recoil spectrum was also obtained for the ${ }^{189} \mathrm{Hg}$ recoils and although low in statistics was very clean as well.

The FMA results were compared to those from the EUROGAM and GAMMASPHERE arrays by analyzing data on the same reaction and ${ }^{160} \mathrm{Gd}$ target as used in the above work. The data from the large arrays were able to produce a ${ }^{189} \mathrm{Hg}$ spectrum, however the gate had is be set blindly since the strongest transition in that nucleus could not be seen without gating, and secondly, the resulting spectrum was not as clean as that produced by the FMA. This comparison demonstrates the superiority of the FMA when looking at weak and unknown recoil channels. Furthermore, this comparison indicates the complementary nature of the FMA and the large arrays and suggests that

†Purdue University, $\ddagger$ I daho National Engineering Laboratory §University of Notre Dame, TVanderbilt University 
the optimur. benefit and power of both may be realized by coupling the GAMMASPHERE array to the FMA.

\section{b.16. Spectroscopy of $186 \mathrm{~Pb}$ with Mass Identification (M. P. Carpenter,}

R. V. F. Janssens, I. G. Bearden, R. G. Henry, D. Nisius, C. N. Davids, T. L. Khoo, T. Lauritsen, H. Penttilä. D. J. Henderson, A. M. Baxter, ${ }^{*}$ A. P. Byrne, ${ }^{*}$ and G. D. Dracoulis*)

It was predicted for some time that deformed prolate structures lie at low excitation energy in the neutron deficient lead nuclei around the neutron mid-shell $(\mathrm{N} \sim 104)$. A recent study in Berlin has identified two prolate-like rotational bands that were assigned to ${ }^{188} \mathrm{~Pb}$ and ${ }^{186} \mathrm{~Pb}$. However, no direct mass identification relating these bands to a specific nucleus was obtained from the experiment. In order to confirm the Berlin claims in the case of $186 \mathrm{~Pb}$, an experiment was performed at ATLAS which measured $\gamma-\gamma$ coincidences gated by the Fragment Mass Analyzer (FMA) to obtain unarnbiguous mass assignments for the $\gamma$-cascade in question.

*Australian National University, Canberra, Australia

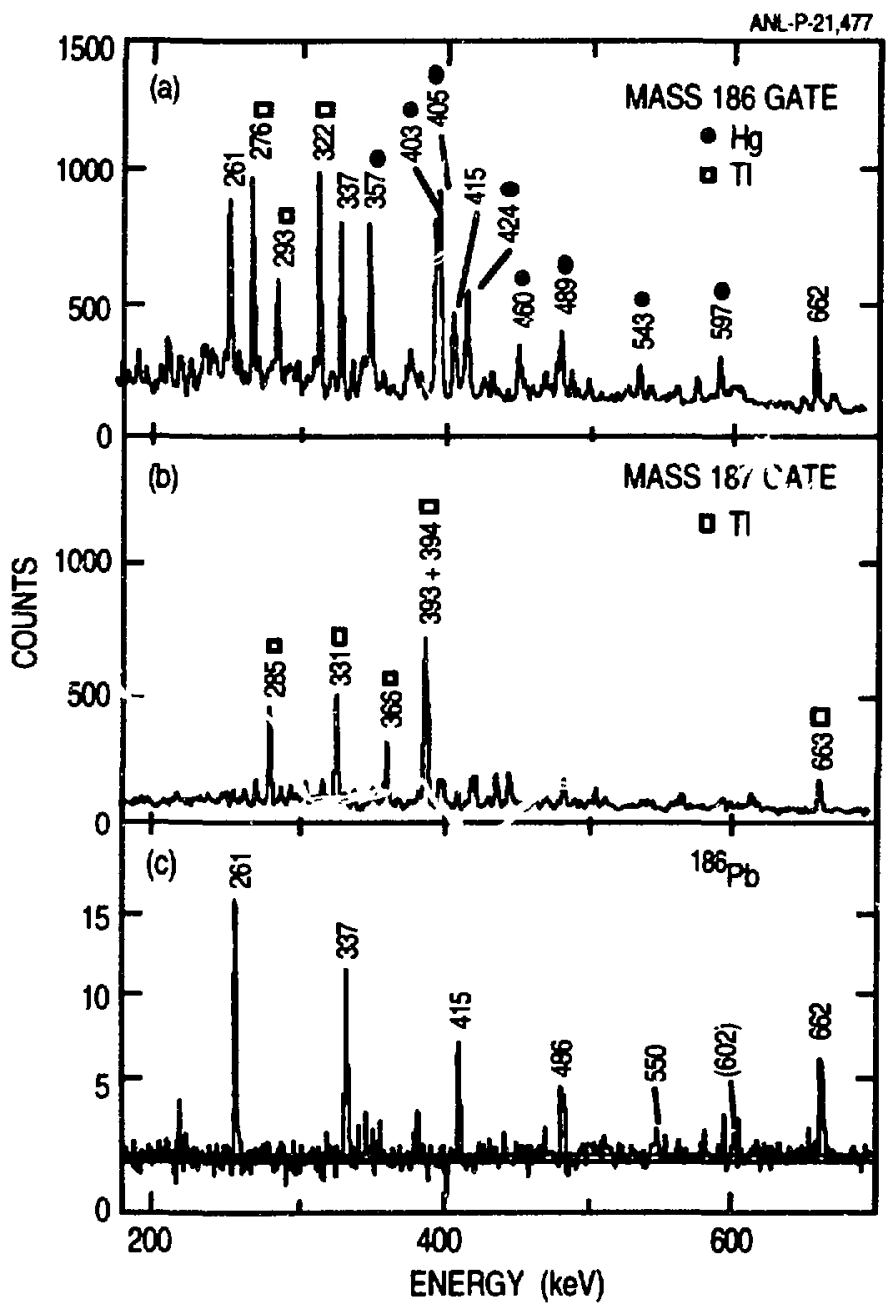

Fig. I-27. (a) and (b) are part of singles $\gamma$ ray spectra gated by FMA position signals; (a) is a sum of gates on the mass-186 peak in the mass spectrum and $(b)$ is a sum of gates on the mass-187 peak. (c) is a sum of projections from the FMA- $\gamma-\gamma$ coincidrnce data with gates on the 261-, 337-, 415- and 662-keV lines, and with gates to select mass-186 evaporation residues. The larger peaks are labelled by their energies and the nucleus to which they are attributed if it is not $186 \mathrm{~Pb}$. 
Excited levels in ${ }^{186} \mathrm{~Pb}$ were populated by bombarding a ${ }^{154} \mathrm{Gd}$ target with a beam of ${ }^{36} \mathrm{Ar}$ at 174 $\mathrm{MeV}$. The target was a $0.38 \mathrm{mg} ;=\mathrm{m}^{2}$ self-supporting foil enriched to $66.5 \%$ in ${ }^{154} / \mathrm{Gd}$. The FMA was set at $0^{\circ}$ to the beam direction to accept evaporation residues recoiling from the target, and $\gamma$ radiation was detected by ten Compton-suppressed Ge detectors placed around the target.

The data were analyzed by constructing an $\mathrm{E}_{\gamma} \mathrm{E}_{\gamma}$ matrix which was in coincidence with mass 186. The analysis has attributed seven transitions which form a cascade to $186 \mathrm{~Pb}$ (see Fig. I-27). The first five transitions were in agreement with the previous work. By plotting the dynamic moment of inertia as a function of rotational frequency $\hbar \omega$ and comparing it to the yrast band in the isotone ${ }^{184} \mathrm{Hg}$, one finds the moments of inertia for the two nuclei to be nearly identical over five of the seven transitions. This supports the view that the ${ }^{186} \mathrm{~Pb}$ band is built on essentially the same prolate structure assigned to the ${ }^{184} \mathrm{Hg}$ yrast band. A similar band was identified in ${ }^{188} \mathrm{~Pb}$, and these two examples provide the first direct evidence for prolate deformation in the lead isotopes.

In addition to this s $\mathrm{tu}$ dy on $186 \mathrm{~Pb}$, the experiment yielded mass identification for both ${ }^{187} \mathrm{Tl}$ and ${ }^{185} \mathrm{Tl}$. The ${ }^{187} \mathrm{Tl}$ identification came from FMA- $\gamma$ coincidences using the reaction ${ }^{155} \mathrm{Gd}\left({ }^{36} \mathrm{Ar}, \mathrm{p} 3 \mathrm{n}\right){ }^{187} \mathrm{Tl}$ at $174 \mathrm{MeV}$, and the ${ }^{185} \mathrm{Tl}$ identification came from FMA- $\gamma$ coincicences using the reaction ${ }^{154} \mathrm{Gd}\left({ }^{36} \mathrm{Ar}, \mathrm{p} 4 \mathrm{n}\right){ }^{187} \mathrm{Tl}$ at $184 \mathrm{MeV}$. Both of these nuclei exhibit prolate bands which are built on $h_{9 / 2}$ and $i_{13 / 2}$ intruder configurations.

b.17. Identification of ${ }^{183} \mathrm{Hg}$ : Identical Bands in ${ }^{183,185} \mathrm{Hg}$ (R. V. F. Janssens, C. N. Davids, I. Ahmad, I. G. Bearden, M. P. Carpenter, R. G. Henry, T. L. Khoo, T. Lauritsen, Y. Liang, H. Penttilä, K. S. Bindra, A. V. Ramayya,ł W. C. Ma,军 B. R. S. Babu, $\neq$ J. H. Hamilton, $;$ L. Chaturverdi, $\neq$ J. Kormicki, $;$ C. Baktash,$\S$ W. Nazarewicz,I and J. A. Sheikh§)

In even-even $\mathrm{Hg}$ isotopes with $\mathrm{A}=180-188$, level structures associated with the oblate groundstate configurations coexist with a number of excited rotational bands associated with a prolate shape. There is also evidence for an excited band with even larger deformation in ${ }^{186} \mathrm{Hg}$, i.e. for a shape intermediate oetween "normal" prolate deformation and superdeformation which was seen in the heavier $\mathrm{Hg}$ nuclei with $\mathrm{A}>188$. As is often the case, the study on odd-even nuclei provides useful information on the nature of the orbitals responsible for the various shapes. Until now, no information was available on the band structures of $\mathrm{Hg}$ odd-even isotopes with $\mathrm{A}<185$. The latter isotopes are important, especially in view of recent calculations which predict competition between low-lying oblate and prolate shapes, some of which are associated with deformations as large as $\beta_{2}$ $=0.6-0.8$.

We identified level structures in ${ }^{183} \mathrm{Hg}$ for the first time from fragment-gamma and gamma-gamma coincidence measurements using the Fragnent Mass Analyzer (FMA) and 10 Compton-suppressed Ge detectors from the Argonne-Notre Dame gamma-ray facility. The ${ }^{183} \mathrm{Hg}$ niclei were produced with the ${ }^{155} \mathrm{Gd}\left({ }^{32} \mathrm{~S}\right.$, in) reaction at a beam energy of $160 \mathrm{MeV}$. The gamma-ray transitions in ${ }^{183} \mathrm{Hg}$ were placed into five bands (Fig. I-28). Two of these were associated with the [624]9/2+ Nilsson orbital and exhibit signature splitting, as expected for $i_{13 / 2}$ excitations built on a prolate shape with moderaie deformation. Two other bands which do not show signature splitting were associated with the [514]7/2- orbital. They exhibit the unexpected feature that both the in-band and intra-band transitions are very close in energy to those of the bands based on the same intrinsic configuration in ${ }^{185} \mathrm{Hg}$. Thus, we found "identical" bands in odd $\mathrm{Hg}$ nuclei with moderate prolate deformation, a feature very similar to that observed for superdeformed bands in the $A=150$ and 190 mass regions and for rotational bands of normal deformation in a few actinide and rare-earth nuclei.

$\ddagger$ Vanderbilt University, §Oak Ridge National Laboratory, qOak Ridge National Laboratory and University of Tennessee 
Calculations using the Woods-Saxon-Strutinsky model were performed in order to understand the observations. It was found that the identical bands are related to the similar behavior of transitions in the excited prolate bands of the respective $182,184 \mathrm{Hg}$ cores and to the absence of significant Coriolis interactions.

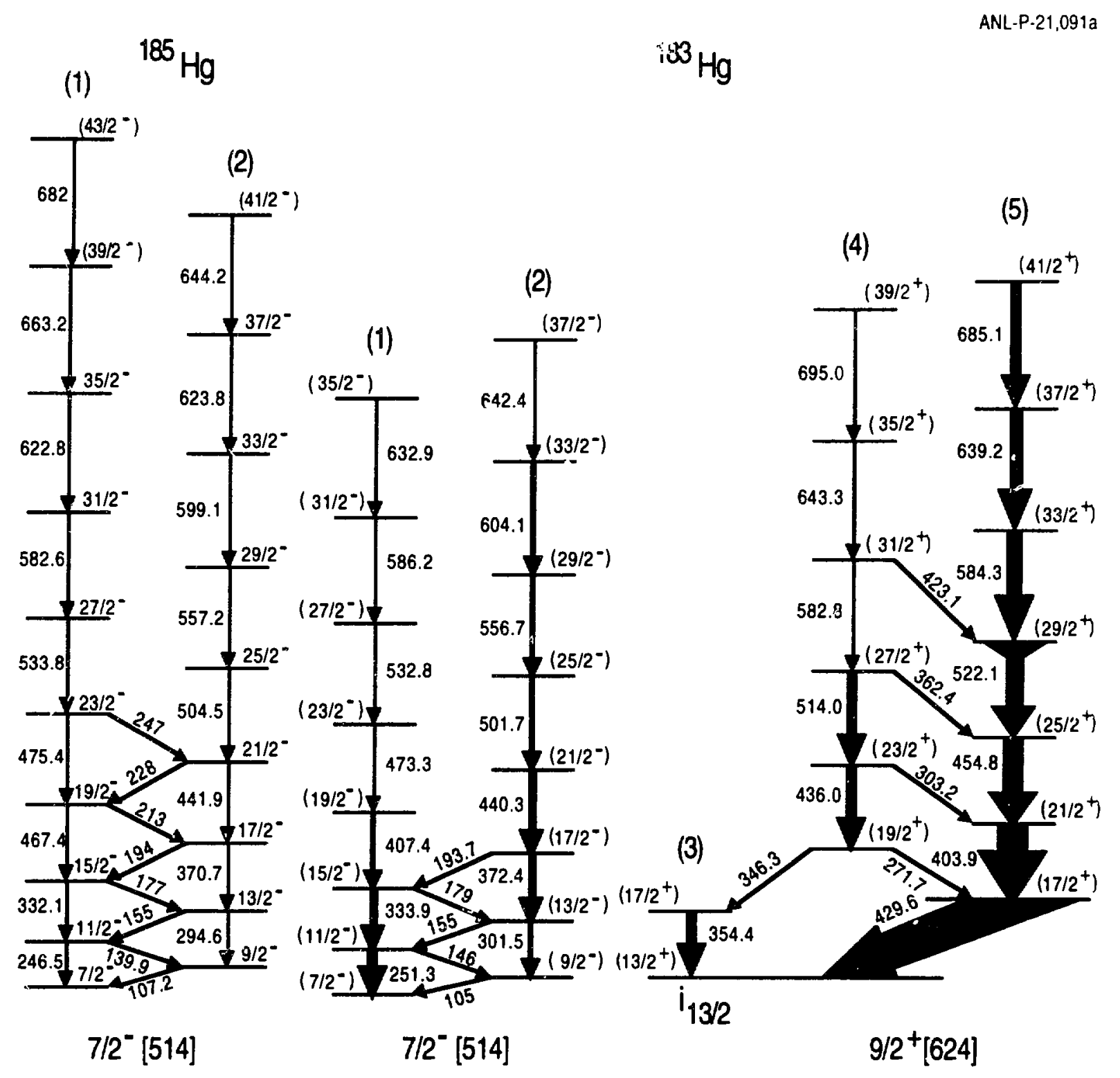

Fig. I-28. The energy leveis in ${ }^{183} \mathrm{Hg}$ observed in this work. A comparison with $185 \mathrm{Hg}$ is also given for bands associated with the $7 / 2-[514]$ orbital. 


\section{b.18. FMA Studies of Proton-Rich Nuclei $\mathbf{N} \sim 82$ by Gamma-ray and Conversion Electron Spectroscopy (D. Nisius, I. G. Bearden, R. V. F. Janssens, I. Ahmad, C. N. Davids, R. G. Henry, T. Lauritsen, T. L. Khoo, H. Penttilä, B. Crowell, D. Henderson, Z. W. Grabowski, $\uparrow$ P. J. Daly, $\dagger$ L. Richter, $\dagger$ R. H. Mayer, $\dagger$ and M. Sferrazza†)}

One of the many powerful uses of the FMA for nuclear structure studies is one where the decay of an isomer is examined at or just behind the focal plane of the instrument following proper mass selection. This technique was applied successfully, for example, to the study of $(\mathrm{hl} 1 / 2)^{\mathrm{n}}$ proton seniority isomers in nuclei with $N-82$ and $Z>64$. Since isomeric decay gamma rays are emitted isotropically, multipolarity information is seldom available from gamma-ray studies and spin and parity assignments are usually made on the basis of calculations and systematics.

Conversion electron measurements allow for multipolarity assignments to be made. Furthermore, the transitions depopulating isomers directly are often of very low energy, i.e. they are highly converted. Thus, by detecting the conversion electrons these transitions can be identified.

A small chamber for the detection of conversion electrons behind the focal plane of the FMA was constructed. It accommodates a catcher foil to stop the mass-selected recoils. It also contains a holder for two silicon PIN diodes operated at room temperature for the detection of the electrons. A first experiment was performed in July employing the reaction ${ }^{94} \mathrm{Mo}+250 \mathrm{MeV} 58 \mathrm{Ni} \rightarrow{ }^{152} \mathrm{Yb}^{*}$ to study the seniority isomers in ${ }^{149,150} \mathrm{Er}$. The decay gamma rays and the conversion electrons were detected respectively by one large Ge detector and by the two silicon PIN diodes mentioned above. In both nuclei $\mathrm{K}, \mathrm{L}$ and $\mathrm{M}$ shell conversion coefficients down to 0.01 were established for all transitions, not only proving that the system is working well, but also establishing the multipolarity of many transitions for the first time. The possibility of improving the detection sensitivity by adding more PIN diodes or of improving the resolution by cooling these detectors are being considered.

†Purdue University 


\section{ACCELERATOR MASS SPECTROMETRY (AMS) AND EXPERIMENTS WITH SECONDARY BEAMS}

Research with AMS concentrates on projects that utilize some of the unique properties of ATLAS: (i) the possibility to start with negative or positive ions from the tandem or ECR-PII injectors, respectively, the latter allowing us to perform experiments with noble gas radioisotopes; (ii) acceleration of the rare isotopes to the high energies required to remove isobaric background by fully stripping; and (iii) acceleration of ions from the whole periodic table to energies sufficiently high to perform isobar separation with the gas-filled magnetic spectrograph.

After the successful detection of $269-\mathrm{yr}{ }^{39} \mathrm{Ar}$ at natural levels at ATLAS a series of experiments studying AMS with $210,000 \mathrm{yr}^{81} \mathrm{Kr}$ have been initiated. These experiments were performed both at ATLAS and at the A1200 reioil separator at MSU.

Accurate half-lives are the backbone of dating experiments. A project to determine the ${ }^{44} \mathrm{Ti}$ halflife has begun. This is of particular interest in connection with a possible detection of ${ }^{44} \mathrm{Ti}$ in supernova remnants by space-based gamma-ray spectrometers.

Experiments in the areas of astrophysics and applied physics atilizing some unique features of secondary beams have begun. The first studies will use the $1.8-\mathrm{hr}{ }^{18} \mathrm{~F}$ isotope. For an astrophysical experiment studying the transition from the hot CNO cycle to the rp-process ${ }^{18} \mathrm{~F}$ will be produced at a high-current cyclotrun and install the radioisotope $18 \mathrm{~F}$ in the negative-ion source of the tandem. For a study of the wear analysis of various industrial components, $18 \mathrm{~F}$ will be produced at ATLAS via the $\mathrm{n}\left({ }^{18} \mathrm{O},{ }^{18} \mathrm{~F}\right) \mathrm{n}$ reaction and implanted into the material of interest. Improvements by a factor of 100 in the sensitivity of wear analysis compared to previous techniques are expected.

a. Detection of ${ }^{81} \mathrm{Kr}$ with Accelerator Mass Spectrometry (W. Kutschera, I. Ahmad, P. J. Billquist, B. G. Glagola, R. Harkewicz, R. C. Pardo, K. E. Rehm, M. Paul,* T. Antaya, $\uparrow$ D. J. Morrissey, $\dagger$ and B. M. Sherrill $\dagger$ )

From a geochemical point of view, the long-lived noble gas radionuclide ${ }^{81} \mathrm{Kr}\left(\mathrm{t}_{1 / 2}\right.$ $=210,000 \mathrm{a}$ ) is an almost ideal tracer. It is produced in the atmosphere by cosmic-ray-induced spallation and neutron actiration of stable $\mathrm{Kr}$ isotopes and is well mixed in the atmospheric krypton reservoir, which is of constant size and has essentially no sources or sinks. Due to the long halflife, short-term fluctuations $\left(<10^{5}\right.$ a) in the cosmogenic production rate should be well smoothed out. ${ }^{81} \mathrm{Kr}$ should also be essentially free from anthropogenic contributions. It is shielded from beta feeding of isobaric fission products by stable ${ }^{81} \mathrm{Br}$, and neutron activation during the periods of atmospheric uuclear weapons testing should amount to less than $1 \%$ of cosmogenic ${ }^{81} \mathrm{Kr}$. The natural ${ }^{81} \mathrm{Kr} / \mathrm{Kr}$ ratio in atmospheric $\mathrm{Kr}$ is $5 \times 10^{-13}$, which is within the typical range of AMS detect:on of cosmogenic radionuclides.

The most interesting application of ${ }^{81} \mathrm{~K}-$ would be the absolute dating of deep ice cores, which presently cannot be performed by any other method. However, the very low concentration of ${ }^{81} \mathrm{Kr}$ in ice ( 1000 atoms $/ \mathrm{kg}$ ice) requires an overall detection efficiency not yet reached with AMS even for the most favorable radionuclides $\left({ }^{14} \mathrm{C}\right)$. Therefore, our first goal was to develop AMS for measuring ${ }^{81} \mathrm{Kr}$ in the atmosphere.

At ATLAS, an experiment utilizing the ECR ion source and the gas-filled spectrograph was performed with $480-\mathrm{MeV}{ }^{81} \mathrm{Kr}$. With neutron activated krypton samples, ${ }^{81} \mathrm{Kr}$ was detected at a level of ${ }^{81} \mathrm{Kr} / \mathrm{Kr}=2 \times 10^{-10}$. However, at the natural level of $5 \times 10^{-13}$, the interference from

*Hebrew University, Jerusalem, Israel, $\uparrow N S C L$, Michigan State University 
${ }^{81} \mathrm{Br}$ was too high. Therefore, an isobar separation with full stripping at $45 \mathrm{MeV} / \mathrm{A}$ with the MSU $\mathrm{K} 1200$ cyclotron was attempted. At this energy, $70 \%$ of ${ }^{81} \mathrm{Kr}$ was fully stripped to $36^{+}$ions in a 18.8-mg thick Be foil. Subsequent analysis of the ions in the A1200 recoil separaior easily separated ${ }^{81} \mathrm{Br}^{35^{+}}$ions, but nuclear reactions of ${ }^{81} \mathrm{Br}$ in the $\mathrm{Be}$ foil produced ${ }^{81} \mathrm{Kr}$ particles which were difficult to distinguish from genuine ${ }^{81} \mathrm{Kr}$ beam particles, below the $10^{-11}$ level. In the future, an experiment will be tried by operating the A1200 in a high resolution mode, where a clean separation of beam particles and reaction products should be possible.

b. Half-Life of ${ }^{44}$ Ti ( I. Ahmad, W. Kutschera, G. Cini Castagnoli,* and M. Paul $\dagger$ )

The half-life of ${ }^{44} \mathrm{Ti}$, which is $\sim 50$ years, is of interest to cosmologists who need an accurate value to determine the production of heavy elements in supernova. The measurement of ${ }^{44} \mathrm{Ti}$ half-life was started two years ago. Three sets of samples - a pure $200 \mathrm{nCi}{ }^{44} \mathrm{Ti}$ sample, a pure $300 \mathrm{nCi}$ ${ }^{60} \mathrm{Co}$ source and a mixed ${ }^{44} \mathrm{Ti}$ and ${ }^{60} \mathrm{Co}$ source of similar strength - were prepared and half-life measurements were started at ANL, Torino and Jerusalem. Each sample is counted with a $25 \% \mathrm{Ge}$ detector for a period of 2 days at an interval of 4 months. The room background is also measured for the same length of time. Measurements are made at two different source-to-detestor distances so that contributions from summing can be estimated. We hope to obtain a half-life with an uncertainty of $5 \%$. The measurements at all three laboratories are in progress.

*Instituto di Cosmogeofisica, CNR Torino, Italy, † Hebrew Úniversity, Jerusalem, Israel

c. Secondary Beam Development with Inverse Kinematic Reactions

(W. Kutschera, J. P. Greene, R. C. Pardo, K. E. Rehm, J. P. Schiffer, D. Berkovits, *

M. Paul,* and T. F. Wang $\dagger$ )

Beams of short-lived ( $s 1 \mathrm{hr}$ ) radionuclides are of particular interesi for studying reactions relevant to nucleosynthesis in so-called "hot" stellar matter. Qualitatively, this is a region where particle reactions on short-lived radionuclides start to compete with beta decay. Since targets of short-lived radionuclides are impractical, beams of these nuclides are required.

We pursue an approach where a beam of $64-\mathrm{sec}^{17} \mathrm{~F}$ is protuced halfway through ATLAS with the reaction $\mathrm{H}\left({ }^{17} \mathrm{O},{ }^{17} \mathrm{~F}\right) \mathrm{n}$. The goal is to obtain enough intensity to study the secondary-beam reaction $\mathrm{H}\left({ }^{17} \mathrm{~F},{ }^{14} \mathrm{O}\right)^{4} \mathrm{He}$ with the split-pole magnetic spectrograph. The inverse of this reaction, ${ }^{14} \mathrm{O}(\alpha, \mathrm{p})^{17} \mathrm{~F}$ mediates the "breakout" from the hot CNO cycle. It was suggested that a measurement of the cross section at an equivalent alpha-particle energy of $2 \mathrm{MeV}$ (sigma $\sim 0.5 \mathrm{mb}$ ) will give valuable information to extrapolate to the interesting region of stellar temperatures around $5 \times 10^{8}$ degrees Kelvin.

The major technical challenge for the production of a ${ }^{17} \mathrm{~F}$ beam with sufficient intensity $\left(10^{5}\right.$ to $10^{7}$ pps), is a hydrogen target that can withstand a primary ${ }^{17} \mathrm{O}$ beam of high brilliance $\left(\sim 1 \mathrm{p} \mu \mathrm{A} / \mathrm{mm}^{2}\right)$. Our studies of a rotating target using Al-coated polypropylene foils have continued. An increase in beam intensity by a factor of 100 (compared to stationary ioils) was achieved. This increase, however, is correlated with a larger gas load which requires additional pumping capacity in the vicinity of the target. We are presently looking into other options such as gas targets or the use of conductive polymers which have better heat conducting properties.

*Hebrew University, Jerusalem, Israel, †̣ Lawrence Livermore National Laboratory 
d. Development Studies for an Experiment with a ${ }^{18} \mathbf{F}$ Beam (K. E. Rehm, J. Nolen, J. Gehring, C. L. Jiang, R. C. Pardo, J. P. Schiffer, J. Nickles, * A. Roberts, and M. Paul $\dagger$ )

As a first step towards experiments with radioactive beams at ATLAS we are presently studying the possibility of using radioisotopes with suitable haif lives using the existing ion sources. These radioisotopes will be produced with high-current accelerators and should have half lives in the range of 2-24 hrs, i.e. short enough to avoid a long-lasting contamination but long enough to allow a measurement with sufficient statistics. Some examples for these isotopes are ${ }^{18} \mathrm{~F},{ }^{48} \mathrm{Cr}$ and ${ }^{76} \mathrm{Kr}$. In a first experiment we are planning to produce ${ }^{18} \mathrm{~F}$ which is a well-known PET isotope produced at the medical cyclotron of the University of Wisconsin, deposit the material electrolytically onto a source holder for the SNICS source, transport it to Argonne and measire the cross section for the reaction $\mathrm{p}\left({ }^{18} \mathrm{~F},{ }^{15} \mathrm{O}\right)^{4} \mathrm{He}$ which is of importance in the astrophysi:al $\mathrm{rp}$ process. Test experiments which study the feasibility of this approach with the reaction $\mathrm{p}\left({ }^{18} \mathrm{O},{ }^{15} \mathrm{~N}\right){ }^{4} \mathrm{He}$ were already performed and a first run with ${ }^{18} \mathrm{~F}$ is planned later this year.

*University of Wisconsin, †Hebrew University, Jerusalem, Israel
e
Study of Wear Analysis with 18 F
(J. Nolen, K. E. Rehm, B.
B. Glagola, J. Greene,
C. L. Jiang, and B. Hillberry*)

We are studying the possible use of low-energy radioactive beams for the wear analysis of various industrial components (e.g. engine parts and orthopedic implants). Previous experiments with ${ }^{7} \mathrm{Be}$ and ${ }^{22} \mathrm{Na}$ studied components at implantation depth of several tens of micrometer. We plan to demonstrate that this technique can be improved to wear studies in the critical first micrometer of the surface layer.

This increased sensitivity can be achieved by implanting a sufficient amount of activity into the material of interest. On the other hand the amount should be small enough so that the intrinsic structure of the material is not changed during the implantation process. These two 1 equirements restrict the possibility for potential candidates to radioisotopes with a sufficiently short half life.

We plan to use ${ }^{18} \mathrm{~F}\left(\mathrm{~T}_{1 / 2}=2 \mathrm{hrs}\right)$ in a first experiment to develop and optimize the technique. ${ }^{18} \mathrm{~F}$ will be produced with an 180 beam via the $p\left({ }^{18} \mathrm{O},{ }^{18} \mathrm{~F}\right) \mathrm{n}$ reaction.

The separation of the $18 \mathrm{~F}$ products from the beam will be done in the split-pole spectrograph using the $9^{+}$charge state. In the focal plane the ${ }^{18} \mathrm{~F}$-ions will be implanted into the surface area of various materials. After implantation the components will be moved to a turntable for tribology measurements. From the amount of ${ }^{18} \mathrm{~F}$ implanted into the surface area, and the expected range distribution, it can be estimated that with this technique a depth resolution for the wear analysis of $1 \mathrm{~nm}$ should be achievable. This corresponds to an improvement by about a factor of 100 compared to previous techniques. The first test runs are planned for summer 1994.

*Purdue University 


\section{OTHER TOPICS}

In addition to the research described in the previous sections, some effort was devoted to other topics, mainly related to the behavior of cooled beams of charged particles contained in storage rings or ion traps.
a.
Measurements with Laser-Cooled Beams at ASTRID
J. S. Nielsen,* O. Poulsen,* and J. P. Schiffer)
(J. S. Hangst,*

The collaboration is continuing to study the properties of very cold laser-cooled beams in the ASTRID storage ring in Aarhus, Denmark. In the past year, new CW ultraviolet lasers were installed that permit the cooling of a beam of ${ }^{24} \mathrm{Mg}^{+}$ions. These have a large advantage over the previously accessible $\mathrm{Li}^{+}$beams, since all the $\mathrm{Mg}$ ions are cooled by the laser, while with the previously studied $\mathrm{Li}$ only the small fraction that was in the isomeric state (about $10^{-4}$ of the beam) could be cooled. The average cooling power for $\mathrm{Mg}$ is about 25000 times that for $\mathrm{Li}$, and amounts to $38 \mathrm{keV} / \mathrm{s}$. This should be ample to cool the bearn, and other limits to beam cooling are likely to occur. As a first step the beam energy was shifted by a small amount as a measure of the cooling force. Only one laser was ready in 1993 and, thus, cooling between two lasers could not yet be studied. However, partial cooling was achieved and the coupling between longitudinal and transverse temperatures established. Next, the beam was bunched in time with a small r.f. voltage and the laser was scanned exerting a force against that of the r.f. electrode, thus trapping the beam in time and velocity spread. The length of the bunches decreases under such cooling and the properties of these bunches are being investigated. In 1994 cooling between two lasers will become possible and the limits to cooling will be better explored. Simulations are being carried out to match the new experimental conditions, particularly with laser cooling.

\section{*Iniversity of Aarhus, Denmark \\ b. Properties of Crystalline Ions (J. P. Schiffer)}

Simulations of the lower limit of temperatures has been carried out in the past but were limited by the amount of computer time available at the ER Crays. In the past year priority was given to cooling a cloud of ions that is an order of magnitude larger than any that were studied in simulations previously. The process of cooling now is more or less completed, for 20,000 ions in isotropic confinement. The resulting spherical cloud exhibits 18 well-defined concentric shells and the properties of this cloud will be investigated in the future.

\section{c. Electron Capture Decay of ${ }^{231} \mathrm{U}$ (I. Ahmad, E. Browne,* K. E. Gregorich,* S. A. Kreek, ${ }^{*}$ D. M. Lee, ${ }^{*}$ and D. C. Hoffman*)}

The electron capture decay of ${ }^{231} \mathrm{U}\left(\mathrm{t}_{1 / 2}=4.2 \mathrm{~d}\right)$ was studied by measuring the gamma-ray and low-energy conversion eiectron spectra of a chemically purified ${ }^{231} \mathrm{U}$ source. The ${ }^{231} \mathrm{U}$ sample was prepared at the LBL 88 -inch cyclotron by irradiating ${ }^{233} \mathrm{U}$ targets with $49-\mathrm{MeV}$ protons. The recoiling ${ }^{231} \mathrm{~Np}$ atoms were carried away from the target area with a helium-jet system and collected on a teflon disc. After the ${ }^{231} \mathrm{~Np}\left(\mathrm{t}_{1 / 2}=49 \mathrm{~min}\right)$ decayed to ${ }^{231} \mathrm{U}$, the uranium was chemically isolated and a thin source prepared. Gamma singles spectra were measured with a LEPS detector and the electrons were measured with a room-temperature Si PIN diode. From these measurements conversion coefficients, EC intensities, and log ft values were determined.

\footnotetext{
*Lawrence Berkeley Laboratory
} 


\section{E. EQUIPMENT DEVELOPMENT AT THE ATLAS FACILITY}

The installation of the various detector systems at APEX was completed and the experimental program with the new devices has started. Initial experience shows that, in essentially all respects, the apparatus performs as conceived and designed. Beams of ${ }^{238} \mathrm{U}$ of intensity up to $5 \mathrm{pnA}$ from ATLAS were used to bombard $1 \mathrm{mg} / \mathrm{cm}^{2}{ }^{181} \mathrm{Ta}$ targets and beam induced positrons were measured with high resolution and essentially zero background. The measured acceptance of the device is close to specifications. The data from the first runs are currently being analyzed but it is already clear that the first operation of the complete device has led to a significant data set.

The Fragment Mass Analyzer (FMA) is now in routine operation and a large fraction of the experiments are performed with this new device. A new implantation system for the FMA focal plane was developed which is crucial for background free studies of nuclei at the limits of stability. These nuclei are identified according to their mass with excellent mass resolution (up to 525:1) in the focal plane and implanted in a Si-detector (segmented into 2304 pixels) located behind the focal plane detector. A subsequent alpha or proton-decay at the implantation site, is correlated with timeof-flight and recoil energy signals, and results in a drastic reduction of the background so that cross sections in the nanob $\mathrm{dr}$ and sub-nanobarn level can be measured.

In the GAMMASPHE. E project, the Physics Division took responsibility for procuring and testing the BGO detectors, for the design and fabrication of the scattering chamber, and for part of the software development. Procurement of BGO detectors is proceeding smoothly and is ahead of schedule, with 83 detectors delivered. Work on the scattering chamber is approaching the final phase and our software effort is about half con pleted.

Other development projects at ATLAS include the double-sided Si strip-detector array and first tests using the gas-filled magnet technique for measurements with radioactive ion beams at very low velocities.

a . Fragment Mass Analyzer Project (C. Davids, B. Back, K. Bindra, M. Freer, D. Henderson, R. Hermann, H. Penttilä, and A. Wuosmaa)

The FMA is now in routine operation, with about half the ATLAS experiments being performed using the FMA. The beam time is split equally between target and focal-plane experiments.

A new implantation system for the FMA focal plane was developed. It uses a $60-\mu$ thick doublesided silicon strip detector (DSSD), with 48,300 - $\mu$-wide strips on each side. The strips on the iront are orthogonal to those on the back, giving a quasi-pixel structure. A total of 96 preamps, fast amps, and QDC channels are used to instrument the detector. Radioactive ions can be implanted in the detector, and their subsequent proton and alpha decays can be studied.

In the commissioning run, the decay of the proton radioactivity ${ }^{147} \mathrm{Tm}$ was observed aft $\mathrm{r}$ producing it via the reaction ${ }^{92} \mathrm{Mo}\left({ }^{58} \mathrm{Ni}, \mathrm{p} 2 \mathrm{n}\right){ }^{\mathrm{P}}{ }^{47} \mathrm{Tn}$. at $261 \mathrm{MeV}$. Each event was tagged as a "recoil" or a "decay", depending on the presence ur absence of a signal from the focal-plane detector. Eäch event was also time-stamped with a clock signal. The $\mathrm{x}$ - and $\mathrm{y}$-coordinates of each recoil event was determined, and when a decay event subsequently occurred in that pixel, the time interval between these two events was stored in a histogram. In this way the decays of both the ground state $(559 \pm 26 \mathrm{~ms})$ and isomericstate $(390+87-76 \mu \mathrm{s})$ were observed. ${ }^{146} \mathrm{Tm}$ was also studied in the same experiment. The system was also used for a number of experiments studying proton radioactivities and alpha emitters in the $\mathrm{A}>150$ region.

A new mode of focusing for the FMA was developed, which allows recoils with up to three charge states to be implanted in the DSSD. The detector is positioned $40 \mathrm{~cm}$ behind the focal plane. at the point where the M/q dispersion of the FMA vanishes. This means that the different misses and 
charge states are brought approximately to the optic axis at this point, resulting in an increased yield. The recoils are distributed uniformly over the face of the detector, making the best usage of the pixel configuration. In addition, with this new configuration, measurements of the recoil energy and time of flight between the focal plane and strip detector can be used to eliminate the background due to scattered beam. In experiments using this new focusing mode, a large increase in sensitivity was attained, which is especially valuable for investigating the decays of short-lived low-yield exotic nuclei.

A silicon detector was used at the FMA focal plane to observe conversion electrons from the decay of high-spin microsecond is $n$ mers in ${ }^{151} \mathrm{Yb}$, with excellent results. Further measurements are p!anned.

A small turbopump was installed at the FMA target chamber, enabling faster turnaround for target changes and giving a better vacuum in the chamber. A target wobbler was also constructed for use in this scattering chamber. It allows target rotation speeds of up to $200 \mathrm{rpm}$, and should enable fragile targets to be bombarded with the more intense beams that are available from the ATLAS positive-ion injector. Another version of the small scattering chamber was constructed at Louisiana State University, incorporating a flange to couple to a cooled high-resolution electron detector. Inbeam measurements of conversion electrons will be attempted in the near future.

b. The ATLAS Positron Experiment (APEX) (The APEX Collaboration - ANL, Chicago, FSU, MSU, Princeton, Queen's, Rochester, Washington, and Yale)

This experiment is designed to study the phenomena associated with the emission of positrons produced in the collision of very heavy ions. In particular, it is aimed at elucidating the origin of the narrow peaks observed in the spectra of positrons and positron-electron pairs measured in coincidence with scattered heavy ions in systems such as ${ }^{238} U+{ }^{181} \mathrm{Ta},{ }^{238} U+{ }^{238} U$ and ${ }^{238} U+$ $232 \mathrm{Th}$, reported by two different groups at GSI Darmstadt, Germany. Despite almost ten years of study by the GSI groups, the phenomenon has remained a rather elusive one and, to date, no wholly consistent explanation of the origin of the narrow lines has emerged.

The most pressing experimental question at the present time concerns a determination of the kinematics of the positron electron coincidence events to determine whether or not the pairs originate from the two-body decay of some previously unknown neutral object, the existence of which in this mass range was hitherto unexpected. Should such an object exist, APEX is designed to provide a measure of its invariant mass to a precision of $25 \mathrm{keV}$. Further, the superior count-rate capabilities of APEX compared to the GSI experiments, coupled with the $100 \%$ duty factor of ATLAS, allows for the accumulation of data at much higher rates than previously possible.

The past year has seen the transition of the experiment from construction and testing to the first physics data taking. There has been a steady progression of the level of operation of the apparatus, coupled with an increase in the intensity of available beams from ATLAS to the point now where essentially all the design goals have been reached. At the time of writing, a two-week-long run has just been completed which has yielded a data set for the ${ }^{238} \mathrm{U}+{ }^{18} \mathrm{~T}$ Ta system with statistics considerably in excess of those typically obtained in the GSI experiments. In the following paragraphs, we outline the progress made during the past year and indicate the likely course of events in the coming twelve months.

During Fall 1992, the first U beams from ATLAS were used to test the focusing properties of the APEX beamline and the bunching of the beam when transported to the APEX target position. These tests were carried out with rather weak beams $(<0.1 \mathrm{pnA})$ and at energies somewhat lower than that required for significant positron production. These beams were also used to test the performance of the heavy-ion counter array which showed performance similar to that expected on 
the basis of the design. Concurrently with this testing, installation of the electronics for the silicon arrays was carried out, limited by the rate at which the final components became available. The integration of the various components comprising the trigger and positron identification was carried out and their correct functioning verified with positron sources.

In early 1993, when the first of the silicon electronics systems was completed, the first measurement of beam-induced positrons was performed. Although both the beam energy and intensity were marginal, it was clear from this measurement that the positron identification scheme using position-sensitive detection of the annihilation photons, was fundamentally sound. Further important information on the level of backgrounds at both the trigger level and in the final analysis was also obtained, allowing for improvements to be made through the installation of shielding around the beam dump. At present, the major source of background triggers arises from the radioactive decay of $T h$ and $K$ found naturally occurring in the walls and floor of the target area.

The first spectrum of beam-induced positrons was obtained from a run in March 1993 with a.5.9$\mathrm{MeV} / \mathrm{u}{ }^{238} \mathrm{U}$ beam incident on a $1-\mathrm{mg} / \mathrm{cm}^{2}{ }^{181} \mathrm{Ta}$ target. In this run, the beam intensity was as high as $4 \mathrm{pnA}$, representing a factor of 100 improvement over the intensities of Fall 1992. The total number of positrons detected in this run was approximately 10,000 with about 1,500 positron-electron coincidence events.

The remainder of the beam-left silicon array was instrumented for a run in May 1993. In this case the silicon array was cooled for the first time, and the resolution for $400-\mathrm{keV}$ electrons from ${ }^{113} \mathrm{Sn}$ decay was found to be approximately $13 \mathrm{keV}$ when the individual spectra for all 216 detector elements of the array were added. In this run, the improvid acceptance resulting from the increased number of silicon elements gave an order-of-magnitude increase in the number of detected positrons and positron-electron pairs, the numbers being 100,000 and 15,000, respectively.

During the following months, the beam-right silicon array was assembled and installed together with the first two modules of the final silicon preamplifier motherboards which incorporated improvements based on experience from the previous runs. This configuration, a full beam-left array and a partial beam-right array allowed for the first time the measurement of back-to-back positron-electron coincidences which was carried out in August 1993. Unfortunately, a power failure during the early stages of the run limited the statistics that could be obtained and further, the 1siolution of some of the silicon detectors deteriorated due to build up of surface contaminants on the cold surfaces of the detectors as a consequence of the power outage. Nevertheless, a data set consisting of 150,000 positrons and 15,000 positron-electron coincidences was accumulated. Based on the reported cross sections, this data set would have been expected to show evidence for the $748 \mathrm{keV}$ sum-energy line, but only at the 2 sigma level.

The delivery of a complete set of the final design silicon preamplifier motherboards in November 1993 allowed for the first data taking with the fully instrumented apparatus in early December. These new electronics allowed for a real improvement in the silicon energy resolution, with overall values of better than $10 \mathrm{keV}$ being observed in measurements with sources. Also installed were new fast amplifiers for the heavy-ion detector array which allowed improved detection efficiency for the low energy target recoils resulting for the forward angle scattering of the beam. Data were taken at two energies, $6.1-$ and $6.3-\mathrm{MeV} / \mathrm{u}{ }^{238} \mathrm{U}$ incident on a $1-\mathrm{mg} / \mathrm{cm}^{2}{ }^{181} \mathrm{Ta}$ target, thus covering the entire energy range over which sharp sum-energy lines have been reported for this system. The accelerator and apparatus performed essentially flawlessly for the entire two-week period with only minimal down time for accelerator problems and the necessary source calibrations of APEX. Over 1,000,000 total positrons were measured, 500,000 at each energy and 250,000 and 170,000 pairs at the lower and higher energy, respectively. This number of events is considerably larger than typically reported from the GSI experiments and the good performance 
of the APEX detector is thus demonstrated. The data are currently being analyzed and should definitely allow conclusions to be drawn regarding the nature of the GSI phenomenon.

The plans for future running of APEX will naturally depend on the outcome of the analysis of the most recent data set. A number of possibilities exist which are:

1) Run on thin (300-microgram/ $\mathrm{cm}^{2}$ ) ${ }^{181} \mathrm{Ta}$ targets to investigate the possible energy dependence of the line production cross section.

2) Study the ${ }^{238} U+{ }^{238} U$ system where the highest fields are produced and for which back-to-back line structure was reported.

3) Study other systems such as ${ }^{238} \mathrm{U}+{ }^{232} \mathrm{Th}$ and ${ }^{232} \mathrm{Th}+{ }^{181} \mathrm{Ta}$ where sharp sum-energy lines were also reported.

4) Run with ${ }^{238} \mathrm{U}$ and ${ }^{232} \mathrm{Th}$ beams on a variety of targets to verify the line phenomenon in singles positron measurements.

These experiments will be scteduled in the coming year and we therefore expect significant physics results to be forthcoming in this period with perhaps the explanation of the positron line phenomenon as well.

Considerable effort has been directed towards understanding the performance of APEX through Monte-Carlo simulations of the response of the various transport and detector systems. These calculations were carried out using the GEANT program which simulates the geometry and all components of APEX. The results of these simulations were compared with the results of measurements with various sources. In general, the agreement between measured and calculated performance is good and also demonstrates that the performance goals outlined in the original proposal were reached. Particularly important among these measurements and simulations was the pair decay from the excited $0^{+}$state at $1.761 \mathrm{MeV}$ in $90 \mathrm{Zr}$ fed in the beta decay of $90 \mathrm{Y}$. This decay simulates quite effectively the signal and background in the beam-induced production of the positron-electron sum energy lines. The beta decay of $90 \mathrm{Y}$ results in the production of roughly one pair per $10^{5}$ electrons, the pair sum energy being $739 \mathrm{keV}$, very close to the energy of the reported lines in the heavy-ion experiments. Further, the determination of the angular correlation of the positron-electron pair allows a test of the ang!e measuring capabilities of the apparatus.

In order to test directly the angle measuring capabilities of APEX, a timed and collimated source of electrons has been devised, using a small PIN diode detector and collimation system placed at the APEX target position. This detector is designed to trigger on one of the two conversion electrons in a cascade decay, such as ${ }^{207} \mathrm{Bi}$, the other being emitted at a well-defined angle to the solenoid axis through a collimator. In this way, the correct functioning of the angle determination of APEX can be verified.

At the end of the last beam time in November, the rotating target wheel was tested under experimental conditions. This device is designed to allow the use of fragile targets which would otherwise fail under the intense heating produced by the energy loss of the beam as it passes through the target. The target wheel contains four targets. The beam is swept off using a signal derived from the phase of the rotating wheel, thus preventing the beam from striking the spokes of the wheel. The rotation is fast enough so that the temperature of any one spot of the target never exceeds the evaporation temperature of the target material. The wheel functioned well, but it was found that the thin evaporated Ta targets used were too brittle to withstand the thermal stresses caused by the heating. A test with $U$ targets showed that these had only rather small amounts of $U$ 
present, most likely caused by the high temperatures experienced by the target substrates during evaporation of the $U$ during fabrication. These problems will be worked on before the next beam time.

A number of upgrades and improvements are planned for APEX during the coming year. These include replacement of approximately one-half of the silicon detectors with multi-guard ring design detectors which have proved superior in performance and ease of operation. Several phototubes on the annihilation detector arrays will be replaced with new tubes with higher gain. We will begin to work on schemes for improving the timing resolution of the silicon detectors and implementing the electronics for allowing detection of positrons and electrons which have energies lower than the threshold of the fast timing discrimirators. In part this may be accomplished by reducing the noise on the fast side of the silicon eler'ronics and also by implementing a discriminator circuit on the shaped silicon energy signals. Lastly, improved segmentation of the heavy-ion counter array will allow higher counting rates. The ability of these detectors to run at high rates is currently the limiting factor in our data-taking capabilities.

\section{c. GAMMASPHERE Activities at Argonne (T. L. Khoo, M. Carpenter, I. Ahmad, J. Falout, R. V. F. Janssens, T. Lauritsen, and D. Visser)}

A powerful third-generation national gamma-ray facility consisting of $110 \mathrm{Ge}$ detectors with BGO Compton suppressors is being constructed at LBL. After 18 months of operation there it will move to another site. This detector system combines calorimetric and multiplicity information with the excellent energy resolution, large efficiency, and high granularity of the Ge detectors. The large number of Ge detectors is essential for high- $(\geq 3)$ fold coincidences. Since each additional fold results in roughly an order-of-magnitude improvement in selectivity, this feature makes it possible to isolate cleanly weak structures, where new physics will undoubtedly lie.

Since GAMMASPHERE represents a national facility, we are committed to participate in its constriction. T. L. Khoo is the current Chairman of the GAMMASPHERE Scientific Advisory Committee (formerly Steering Committee) which follows, and provides advice on, the construction of GAMMASPHERE. R. V. F. Janssens has been Chairman of the Users Executive Committee since its inception.

Through our activities in R\&D, planning committees, and the Steering Committee, ANL has played a major role in securing funding for the project and in defining the instrument. Now, with the project in the construction phase, we are vigorously participating in GAMMASPHERE construction. Argonne is responsible for: (i) all aspects of the BGO detectors, including specifications, design, procurement, testing and mechanical verification; (ii) a substantial portion of the front-end VME software, including all asperts which involve Ge and BGO detector parameters (calibration, trapping corrections, threshold levels, etc.), as well as event simulation and user interface; (iii) target chambers for both the early implementation phase (consisting of 30 Comptonsuppressed spectrometers) and the final phases. Procurement of BGO detectors proceeds smoothly and is ahead of schedule, with 83 detectors delivered. Testing of these detectors has represented our present major effort, but the bulk of testing will now be done by Stony Brook. Work on the final-phase scattering chamber is approaching the last phase. Our software effort is about half completed.

Earlier Argonne GAMMASPHERE contributions include: (i) providing funds for purchasing prototype Ge and BGO detectors; (ii) procuring all prototype detectors; (iii) testing of all prototype detectors including measurements of the combined performance of the Ge and BGO detectors as a Compton-suppressed spectrometer; (iv) developing software methods to correct for ballistic deficit and charge trapping at neutron-damaged sites in Ge detectors; (v) participating strongly in development of the detector configuration, electronics, computer hardware/software, and mechanical support design of GAMMASPHERE - for example we proposed the electronic- 
honeycomb design; (vi) continuing to suggest ways to improve the performance of GAMMASPHERE, e.g. to avoid the degradation due to neutrons and a segmented readout scheme for coaxial Ge detectors; and (vii) participating in writing the GAMMASPHERE Baseline Review Document (May 1991) and the GAMMASPHERE functional requirements, which define the capabilities of the instrument.

\section{c.1. Testing and Procurement of the BGO Compton-Suppression Detectors \\ (M. P. Carpenter, I. Ahmad, G. A. Annan, A. Bernstein, S. Harfenist, \\ R. V. F. Janssens, T. L. Khoo, and D. Visser)}

GAMMASPHERE, the national $\gamma$-ray facility, when completed will consist of 110 Comptonsuppressed Ge detectors. The bismuth germanate (BGO) Compton-suppression detector system for each Ge detector consists of one tapered hexagonal BGO side shield and one slotted BGO back plug. Due to the geometry of the array, three types of annular shields will be required. These types are referred to as $B, C$ and $D$, and the array will consist of 60,30 and 20 of these units, respectively.

Shield types B, C and D are divided into six optically separate sections, each with its own pair of photomultiplier tubes.

Argonne has taken responsibility for the procurement and testing of the BGO Comptonsuppression units. The procurement of $\mathrm{GO}$ detectors for GAMMASPHERE is proceeding extremely well and ahead of schedule. As of January 1994, we have received 38 B-type, $30 \mathrm{C}$ type, 15 D-type and 82 backplug detectors from the two vendors. All but five of these detectors were delivered to ANL for testing. Recently, five B-type detectors were delivered to the University of Stony Brook in order for testing of detectors to begin at this institution. Approximately twenty-five B-type detectors will be tested at Stony Brook.

The vast majority of crystals meets or exceeds the performance criteria established previously. However, several of the more recent detectors delivered by both vendors have had problems meeting the uniformity specification. Several detectors were returned and it is anticipated that the uniformity problem will be easily solved. Examination of the physical dimensions of the detectors using test molds and a coordinate measuring machine (CMM) has shown that the BGO detectors conform weli to the tight mechanical specifications.

\section{c.2. Target Chamber for GAMMASPHERE (M. P. Carpenter, J. Falout, I. Ahmad, R. V. F. Janssens, and T. L. Khoo)}

As one of our responsibilities for GAMMASPHERE, we have taken on the task of designing and constructing two target chambers and associated beamlines to be used with the spectrometer. The first chamber is currently in use with the early implementation phase of GAMMASPHERE, and consists of two spun-Al hemispheres welded together giving a wall thickness of 0.063 inches and a diameter of 12 inches. The top and bottom of the sphere have been lopped off in order to take into account the geometry defined by the support. The flat surfaces at the top and bottom are fitted with lids, and the target is supported from the top lid. The total height of the chamber is 8.875 inches.

The second chamber will be used in conjunction with the final support frame tor GAMMASI'HERE. When completed, the chamber will be a 14-inch-diameter sphere and will take up most of the space available between the target and heavimet shielding. Limited access to the inside of the chamber will come from the side where a six-inch opening has been made available. Five ports will be placed on the chamber, all located at pentagon positions and out of the view of all Ge detectors. Two of these ports will attach to the beamlines, and one will be used for attaching ine chamber to a support strut. A target drive will be mounted inside the support strut and will be 
remotely controlled. A port directly opposite the support port will also be made available. For most experiments it is envisioned that the target ladder will be driven out through this port in order to mount or detach it. The final port can be used for viewing the target. Specification drawings are completed and final approval is pending. Construction will begin soon.

c.3. GAMMASPHERE Software Development (T. Lauritsen, I. Ahmad, M. P. Carpenter, R. V. F. Janssens, and T. L. Khoo)

ANL responsibilities for the GAMMASPHERE project include the development and testing of software for a range of calibration tasks for the detector array. Among other things, we developed routines for the energy calibration of both the germanium detectors and the BGO Compton suppressor shields. The latter involved the use of Compton scattering of gamma-rays from the germanium crystals into the BGO crysials which are shielded from gamma-rays emiited at the target position in the center of the array. Thus, the BGO crystals can be calibrated even with heavymet shields in front of the BGO detectors.

We also developed techniques to use a 2-dimensional energy-time histogram to reject neutron events efficiently in the germanium detectors without losing efficiency at low energies. The method was automated so that a large number of detectors with quite differeñi timing characteristics can be processed without much manual adjustment. We plan to expand this technique to minimize false suppression by neutron hits in the BGO shields. In this way we can improve the high-fold efficiency of the GAMMASPHERE array.

Graphical User Interfaces (GUI) using TCL/TK were developed for the germanium energy calibrations, and "point-and-click" interfaces were also developed for other utilities. This will make it easy for users of the array to perform calibration tasks without the necessity of reading manuals since the tasks are guided by easy-to-understand pull-down menus.

We are currently involved in the finalization of the EPICS interface for the slow signal controls of the GAMMASPHERE array. Finally, utilities were created to read and write spectra from the front-end filter formatters to spectra in the ORNL forma:. The spectra can then be displayed in the ORNL data display and manipulation package, whict, is part of ORNL's contribution to the GAMMASPHERE project.

\section{c.4. Software for Analyzing High-Fold Coincidence Data (T. Lauritsen, B. Crowell,} I. Ahmad, M. P. Carpenter, R. Henry, R. V. F. Janssens, T. L. Khoo, and D. T. Nisius)

The data froh 1 most gamma detector arrays comes in a format that is not easily readable. Moreover, the formats from the various arrays are all different and quite often the data is not calibrated. It is therefore advantazeous to preprocess and compress the data from the primary data tapes to a simplified common format before further processing. We developed such a utility and used it on data acquired at both the European EUROS.AM detector array and the United States GAMMASPHERE array in its early implementation phase. We expanded the utility to do a dynamic energy calibration of the germanium signals in addition to processing of 2-dimensional energy-time information. Thus, in the subsequent data analysis very little, if any, processing must be done to the data from the preprocessed high-density tapes. This makes the development of sorting algorithms to take advantage of the high fold of the events from the new generation arrays much easier.

The use of three-dimensional histograms, i.e. coincidence cubes, provides a great deal of power and flexibility for analyzing three-fold data. We built on the work of $J$. Kuehner and G. Hackman, who wrote software for sorting data into such a histogram, in a compressed format. 
We developed a method for creating one-dimensional gated histograms from a cube with the proper background subtraction. Our software incorporates a fully automatic algorithm for eliminating the contributions to the gated spectrum of the smooth background underlying the coincidence peak on which one is gating. A full propagation of errors is carried out, to allow peaks in the final 1dimensional gated spectrum to be fitted with the appropriate error bars. We used the software successfully in the analysis of several datasets from GAMMASPHERE, and are currently preparing a description of this work for publication. The algorithm can be generalized in a very natural way to four-fold and higher coincidence data.

We also developed software to search for superdeformed bands in a gamma-ray triples dataset. Automatic search routines offer speed and convenience; in turn, the speed makes it practical to explore a variety of search patterns. We used the algorithm successfully to find four previously unknown superdeformed bands in ${ }^{151}$ Dy (as well as several superdeformed bands which were independently discovered in a concurrent search by hand). We are currently considering ways to improve the searching algorithm, and are studying the possibility of implementing a similar algorithm for four-fold coinciderce data, on the Argonne Mathematics and Computing Division's SP-1 parallel processing computer.

Mostly it is desirable to sort the high-fold data from the new large detector arrays into cubes ( $\mathrm{E}_{\gamma}$ vs. $E_{\gamma}$ vs. $E_{y}$ ). However, to find the multipolarities of the gamma rays it is necessary to sort the data according to the detector angle, and it becomes impossible to generate cubes for all angle combinations due to a limited amount of disk space. We developed software to make primarygated angle-sorted matrices, enabling us to do such analyses and at the same time exploit the cleanliness of double-gated data. Algorithms were developed for a correct background subtraction of the data. The software package can use non-linear energy scales so that data up to high energies can be retained in the matrices without loss of resolution. The use of this software package on data obtained from the EUROGAM array has enabled us to isolate the spectrum of $\gamma$ rays following decay of the superdeformed hand in $192 \mathrm{Hg}$.

\section{d. Status of the Argonne Notre Dame BGO Gamma-Ray Facility at ATLAS}

(R. V. F. Janssens, M. P. Carpenter B. Crowell, J. Falout, J. Joswick, T. L. Khoo, R. G. Henry, T. Lauritsen, J. Ti: $i_{2}$, P. Wilt, I. G. Bearden, and D. Nisius)

The gamma-ray facility at ATLAS consists of (a) a $4 \pi$ gamma-sum/multiplicity spectrometer with 50 BGO hexagonal elements (inner array) and (b) 12 Compton-suppressed germanium detectors (CSG) external to the inner array. During the past year the effort related to this facility continued on several fronts.

-- Because of neutron damage, annealing was performed on all Ge detectors. Four of these were annealed twice. The performance of the detectors was recovered in every case.

-- Maintenance and repairs had to be performed on several electronics modules and, in particular, on some of the CAMAC units. None of these problems affected an experiment for more than a couple of hours.

-- Preventive maintenance was performed on the $\mathrm{LN}_{2}$ filling system (inspection of all filling lines and check of the various functions of the control modules).

-- Software programs for automatic gain matching and other control parameters of the array elements and CSGs were upgraded.

-- 10 of the CSGs were moved to the FMA for long periods of time and were used in conjunction with this device. Such a move takes about 1 day, does not require that the Ge detectors be warmed up, and has not resulted in any noticeable loss in performance of the CSGs. 
e. A Particle Ball for GAMMASPHERE (R. R. Betts, A. H. Wuosmaa, C. J. Lister, and D. Blumenthal)

The availability of the new generation of gamma-ray detector arrays, such as GAlAMASPHERE and EUROGAM, opens up many new and exciting possibilities for charged-particle gamma-ray coincidence experiments which will aJdress new aspects of nuclear structure and reaction physics. Examples of these experiments include properties of highly-exrited high-spin nuclei and the study of exotic states in light nuclei. To tully exploit the capabilities of the gamıma detection arrays, the charged-particle detection system should also possess optimum efficiency and resulution in both energy and position, which leads us to the concept of a "Particle-Ball". In addition to its use in conjunction with gamma arrays, such a device would also have powerful capabilities in a standalone mode particularly in experiments with radioactive beams whose low intensity will make highefficiency detection systems imperative.

We started the conceptual design and specification of such a device. One conclusion from these early discussions and planning is that the device needs to be rather fiexible to allow many different geometric configurations, optimized to the needs of specific experiments. We have therefore been led to the concept of detector modules, each with its own electronics and readout, any number of which can be configured in the geometry dictated by the requirements of each type of experiment. The basic element will most likely consist of a $5 \times 5 \mathrm{~cm}^{2}$ double-sided silicon strip detector with 25 strips on each face, thus giving an effective 625-pixel detector with 2-mm position resolution in both $\mathrm{X}$ and $\mathrm{Y}$ coordinates. We are starting to evaluate the options available for readout of such a detector using an integrated circuit preamplifier, ADC, and logic modiles similar to those commonly used in high-energy physics experiments with silicon strip detectors. Orie particularly interesting pc ssibility is that the electronics currently being developed for the RHIC experiment, PHOBOS, may be useful in this application. In PHOBOS, the need to determine an energy loss of up to $10 \mathrm{MeV}$ for charged particles which pass through their detectors is in the same range as that required for low-energy experiments. The resolution that can be obtained with these electronics is of course a crucial question. In the coming year we will start evaluation of various types of integrated circuits in the environment of low-energy experiments. Assuming that this proceeds in a satisfactory manner, the community of interested users vill most likely develop a proposal for funding of the complete device.

\section{f. The Study of Fusion Reactions with a Gas-Filled Magnetic Spectrograph} (K. E. Rehm, J. Gehring, D. Henderson, W. Kutschera, and M. Paul†)

We started a program to measure excitation functions of fusion yields using a gas-filled spectrograph. Normal $\Delta E-E$ techniques are limited by the count rate the detectors can handle. We built a parallel-grid avalanche counter (PGAC) for the focal plane of the Enge split-pole spectrograph which was optimized for fusion measurements. It has a 1- $\mu$-thin Mylar entrance foil, an increased vertical acceptance, and a divided anode which allows the detection of elastically scattered particles and evaporation residues independently in the two halves of the detector. The efficiency of the detector system for fusion cress section measurements was determined by detecting evaporation residues (ER) from the systems ${ }^{32} \mathrm{~S}+{ }^{64} \mathrm{Ni},{ }^{58} \mathrm{Ni}+{ }^{64} \mathrm{Ni}$ and ${ }^{64} \mathrm{Ni}+{ }^{100} \mathrm{Mo}$. A comparison with cross sections measured previously for these three systems showed good agreement. Monte Carlo calculations with the modified program RAYTRACE were performed to study the optimum conditions for the gas-filled magnet technique. It appears that the separation between elastically scattered particles and evaporation residues is largest at the highest possible gas pressures, especially at low bombarding energies. These results were confirmed by the measurements.

$\lceil$ Hebrew University, Israel 


\section{g. Study of the Particle Identification for Low-Z Ions at Energies Below 1 MeV/u (K. E. Rehm, C. L. Jiang, and M. Paul*)}

Many of the planned experiments with secondar y beams will use inverse reaction kinematics with a hydrogen target consisting of $\mathrm{CH}_{2}$. As a consequence, the number of reaction products increases because reactions involving $\mathrm{H}$ and $\mathrm{C}$ can occur. Thus, in many cases the mass and charge of the outgoing particles has to be determined and this can be difficult if the energy of the ions is below 1 $\mathrm{MeV} / \mathrm{u}$. For a planned experiment with ${ }^{18} \mathrm{~F}$ beams an additional complication arises because the $\beta^{+}$ decay of ${ }^{18} \mathrm{~F}$ to ${ }^{18} \mathrm{O}$ causes the beam purity of the accelerated beam to change during the experiment. We, therefore, started to investigate if a gas-filled magnet can be used for particleidentification at very low velocities and low $\mathrm{Z}$.

The experiment was performed with a $600-\mathrm{keV} / \mathrm{u} 180$ beam from the tandem accelera:or using the split-pole spectrograph which was filled with $\mathrm{N}_{2}$ at $\mathrm{p}=0.5$ torr. The particles were detected in the focal plane with a $x$-y-position-sensitive parallel-grid avalanche counter. Using ${ }^{12} \mathrm{C},{ }^{14} \mathrm{~N},{ }^{15} \mathrm{~N}$, ${ }^{18} \mathrm{O}$ and ${ }^{19} \mathrm{~F}$ ions with energies of $\sim 500 \mathrm{keV} / \mathrm{u}$ generdted in various nuclear reactions, the particle separation using magnetic rigidity and time-of-flight measuremeuts was investigated. A preliminary analysis indicates that the separation of ${ }^{15} \mathrm{~N}$ from ${ }^{18} \mathrm{O}$ in magnetic rigidity is such that a complete separation from the beam particles is possible. First experiments with different gases ( $\mathrm{He}, \mathrm{CO}_{2}, \mathrm{Ar}, \mathrm{Kr}$ ) were also performed to study the optimum conditions for the gas-filled magnet technique. A comparison with the results of Monte Carlo simulations is planned for the immediate future.

\section{*Hebrew University, Jerusalern, Israel}

\section{h. Radiation Safety at Low-Energy Heavy-Ion Accelerators (B. B. Back)}

The beam-induced radiation hazards at lowv-energy heavy-ion accelerators are evaluated on the basis of nuclear physics principles. The flux, energy and angular distribution of neutrons and $\gamma$ rays generated by stopping the beam in various materials are calculated and compared to available experimental data. Beam interactions with the residual gas in beam pipes are rot considered. The induced radioactivity of beam-stopping components is estimated.

\section{i. Physics Division Computer Facilities (D. R. Cyborski and K. M. Teh)}

The Physics Division maintains several computer sy'stems for data analysis, general-purpose computing, and word processing. Prior to this year, this w'sk :vas concentrated primarily on two VMS VAXclusters within the Division. Over the past year, these capabilities were broadened considerably with the addition of several RISC-based UNIX workstations.

The main Divisional VAXcluster which consists of two VAX 3300s, configured as a dual-host system, serves as boot nodes and disk servers to seven other satellite nodes consisting of two VAXstation 3200s, three VAXstation 3100 machines, a VAX-11/750, and a MicroVAX II. There are three $6250 / 1600$ bpi 9-track tape drives, six 8-mm tapes and about $9.1 \mathrm{~GB}$ of disk storage served to the cluster by the various satellites. Also, two of the satellites (the MicroVAX and VAX11/750) have DAPHNE front-end interfaces for data acquisition. While the system has operated reliably for the past year, small improvements continue to be made. These include relieving some of the word-processing tasks with Macintoshes and PCs and the addition of a direct tape-to-tape copying facility on one of the satellite nodes. Although not part of the main cluster, a DEC 3000 Alpha machine obtained for data acquisition is also available for data replay.

Computers within the Division have access to the following networks: Argonne DECnet, Eitnet, HEPnet, ESnet, and Internet. The weak-interactions and medium-energy physics groups operate a VAXcluster consisting of two VAXstation $4000 / \bmod 90$, of one VAXstation 3200 , one 
VAXstation 3100, and a MicroVAX II. The weak-interactions MicroVAX II is equipped with a 9 track tape drive and a DAPHNE data-acquisition system.

The ATLAS and Dynamitron VAX-11/750s continue to operate reliably. The Dynamitron VAX (also a pari of the VAXcluster) is used routinely for data acquisition and replay. The ATLAS VAX (not clustered) provides data acquisition for two sisinltaneous i'sers at the ATLAS accelerator, as well as replay when CPU time and memory are available. The VAX $\angle n 00$, acquired as a dedicated data acquisition computer for the APEX experiment, was also improved with added memory, an additional tape drive, and more hard disk storage. It is also being used heavily for data replay.

In addition to the VMS VAX clusters, the Division also maintains three UNIX clusters. The Theory Group has an IBM RS/6000 workstation which serves several X-windows terminals. The Medium-Energy Group has two Silicon Graphics workstations which are used for data analysis and a Sun IPX Sparcstation which is used for CAD/CAM. The Division's main UNIX cluster, which consisted of 2 Sun Sparcstations, saw the addition of three DEC 3000/300L workstations this past year. Efforts to configure these five machines in a clientiserver type configuration were completed successfully. They are now being used regularly for data analysis and GEANT detector simulation studies. Work continues to install and develop software on the UNIX machines necessary for data acquisition and Division-specific tools for general computing.

j. Data-Ãcquisition Systems (D. R. Cyborski and K. M. Teh)

DAPHNE, the data-acquisition system developed for ALAS, is used routinely for experiments at ATLAS and the Dynamitron. In addition to DAPHNE, the Division also maintains 2 MSU/DAPHNE systems. The MSU/DAPHNE system is a hybrid data-acquisition system which combines the front-end of the Michigan State University (MSU) DA system with the traditional DAPHNE back-end. The first such system was obtained for the APEX experiment and was used there exclusively for the past year. A second MSU front-end, purchased as a backup for the APEX experiment, is now being installed as a fully-independent second MSU/DAPHNE system with the procurement of DEC 3000 Alpha host computer.

An improvement to the Budget-Acquisition DAPHNE (BAD) system was also completed this year with the design and construction of an auxiliary module. The BAD system was designed to handle offline, low-rate, detector test-type setups and was used in the testing of detectors for the GAMMASPHERE project. The auxiliary module which plugs into the CAMAC backplane was designed to simplify the handling of external input and output signals and triggers to the BAD system.

\section{k. Nuclear Target Development (J. P. Greene and G. E. Thomas)}

The Physics Division operates a target development laboratory that produces the thin feil targets needed for experiments performed at the ATLAS and Dynamitron accelerators. Targets are not only produced for the Physics Division but also for other divisions and occasionaily for other laboratories and universities.

In the past year, numerous targets were fabricated either as self-supporting foils or on various substrates, many of these targets for the first time. Targets produced included $\mathrm{Ag}, \mathrm{Au},{ }^{11} \mathrm{~B}$,

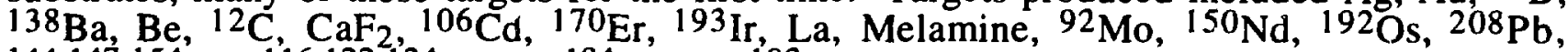
$144,147,154 \mathrm{Sm}, 116,122,124 \mathrm{Sn}, \mathrm{Ti},{ }^{184} \mathrm{~W}$, and $182 \mathrm{WO}_{3}$. Stretched targets of $\mathrm{Al}, \mathrm{Au}, \mathrm{C}$, and $\mathrm{Ni}$ along with metallized Mylar were produced for an atomic physics run at ATLAS. Targets of ${ }^{154} \mathrm{Sm}$ and ${ }^{122} \mathrm{Sn}$ were made onto stretched gold backing foils for Notre Dame for use in lifetime measurements. 
An increased output of foils fabricated issing our small rolling mill included targets of $\mathrm{Ag}, \mathrm{Al}, \mathrm{Au}$, C, ${ }^{106} \mathrm{Cd}, \mathrm{Co},{ }^{50} \mathrm{Cr}, \mathrm{Cu}, 54 \mathrm{Fe}, 154,155,156,160 \mathrm{Gd}, \mathrm{Ho},{ }^{176} \mathrm{Lu}, \mathrm{Mg},{ }^{92,94} \mathrm{Mo}, 58,64 \mathrm{Ni},{ }^{208} \mathrm{~Pb}$, ${ }_{110} \mathrm{Pd}, \mathrm{Sc}, \mathrm{Ti}, \mathrm{Zn}$ and $92,94 \mathrm{Zr}$. Large quantities of APEX targets were prepared by rolling, mainly $1-\mathrm{mg} / \mathrm{cm}^{2} \mathrm{Ta}$ foils but also $\mathrm{Ag}$ and $\mathrm{Au}$. A series of rare-earth targets, $\mathrm{Ho}, \mathrm{Lu}, \mathrm{Tb}$ and $\mathrm{Tm}$ were needed for testing the heavy-ion counters. Rolling has become the method of choice for most targets used in FMA experiments where the required aerial densities rang from 0.5 to $0.7 \mathrm{mg} / \mathrm{cm}^{2}$ and even up to $1 \mathrm{mg} / \mathrm{cm}^{2}$. The same is true for gamma-ray experiments, where the aerial densities needed decrease to $0.3 \mathrm{mg} / \mathrm{cm}^{2}$. A large effort will be required to prepare $0.3-\mathrm{mg} / \mathrm{cm}^{2}$ Ta targets for APEX.

With the early implementation of GAMMASPHERE now operational, an increase in target requests for these experiments is noticed. Targets of "stacked" ${ }^{160} \mathrm{Gd}$ and ${ }^{122} \mathrm{Sn}$ foils were produced for Argonne runs at Berkeley. Sandwiched targets of Au- ${ }^{138} \mathrm{Ba}-\mathrm{Au}$ and $\mathrm{Au}-{ }^{150} \mathrm{Nd}-\mathrm{Au}$, both very reactive elements, were prepared successfully and transported under vacuum or argon to Berkeley for GAMMASPHERE experiments. Targets supplied for other institutions included ${ }^{65} \mathrm{Cu},{ }^{156} \mathrm{Gd}$ and ${ }^{58} \mathrm{~N}_{1}$. This support will increase in the future.

In all, approximately 856 targets were prepared for these various experiments during the past year.

As part of ATLAS support, carbon stripper foils of $2 \mu \mathrm{g} / \mathrm{cm}^{2}$ for use in the Tandem as well as other thicknesses for additional stripping are being produced routinely by the target lab. A total of 961 stripper foils of various types were prepared in calendar year 1993. As ATLAS is now on a 7day schedule, the demand for this support is beginning to increase.

The target development laboratory includes state-of-the-art equipment used for thin-film deposition. The available techniques consist of multiple resistive heating, focused ion-beam sputtering, electron beam and electron bombardment evaporation. The evaporators are maintained under high vacuum and each vessel contains a quartz-crystal film-thickness monitor with deposition rate indicators. Also included are movable shutters, quartz-lamp substrate heaters and thermocouple iemperature sensors. This allows for complete process monitoring during target deposition.

A wide-beam Saddle Field Ion Source was pu chased for the larger beam spot size necessary for new ion milling applications. This source will auso provide increased sputtering rates during target depositions.

Auxiliary equipment used for target development includes a glow discharge apparatus for plasma deposition, mainly of carbon foils. Future plans call for reactivation of this system possibly using a diffusion pump to augment our purchasing of carbon stripper foils. Also present are a small rolling mill, mentioned previously, an alpha-particle thickness gauge (see below), inert atmosphere glove box, laminar flow clean bench, a reduction furnace, and a variety of precision balances.

The IBM PC-XT laboratory computer is used extensively for a liumber of purposes. File archives maintained on this system include all targets produced, dating back to 1978. Computer listings can be generated for inventories of all stable isotopes and chemicals maintained by the target lab. An ADC board and associated software allows for acquisition and analysis oî alpha-particle energyloss measurements needed for film thickness determinations.

A target storage facility is in operation for maintaining, under high vacuum, those targets which can readily oxidize in air. This system utilizes a turbo pump and employs computer-controlled circuitry to prevent targets from exposure to atmosphere during power interruptions. Plans call for this equipment to be installed in ATLAS Area II for the storage of these reactive targets which have been activated by the beam and therefore kept within the controlled Area of ATLAS. A second, 
additional turbo-pumped chamber is now in routine use for target storage. This system uses electronically controlled valves for preserving the targets under high vacuum.

There also exists a bank of vacuum desiccators connected to a mechanically-pumped manifold for \lrcorner se by individua! experimenters. The mechanical pumping system has been upgraded to include an emergency shut-off valve which prevents venting of the desiccators to atmosphere during power interruptions. A second, similar system will be constructed at ATLAS for storage of targets which have been activated by the beam during experiments.

The low-level radioactive target laboratory dedicated to the production of radioactive target foils has seen increased use over the past year mainly in preparation of calibration sources for APEX. The target lab has been called upon for the preparation of the many, various backings needed for source production using the technique of direct spreading. Plans for the smaller evaporator system contained in the lab, constructed for close proximity evaporations of higher activity materials, call for increasing the evaporation yields obtained during radioactive source depositions. 


\title{
F. ASSISTANCE TO OUTSIDE USERS OF ATLAS
}

\author{
B. G. Glagola
}

There is continuing strong interest by outside users in doing research at ATLAS, with outside users involved in about $95 \%$ of all experiments performed in FY 1993. The user assistance program is essential in facilitating effective performance of research by outside scientists.

The outside user involvement continued 10 increase from that in 1992 . A user liaison physicist continues to play a key role in channeling assistance to outside users. The major components of his responsibility are: (1) to provide users with technical information about ATLAS and its experimental systems, and to provide instruction in its use; (2) to assist outside users in all aspects of initiating and planning an experiment; (3) to provide the needed information and organizational assistance to committees, workshops, and other meetings involving outside users; (4) to the extent that is appropriate and feasible, to assist users in the actual performance of experiments; (5) to provide instruction and help with the use of computer hardware and software; (6) to instruct the users in the safety procedures to be followed when using the ATLAS facility; (7) to assist in coordinating the operation of the technical support group; and (8) to provide an interface between the user and the technical support and ATLAS operation groups.

Two PAC meetings were held during FY 1993. The Program Advisory Committee (PAC) for ATLAS (nominally consisting of five members from other institutions and two from Argonne) continues to meet regularly during the year. PAC meetings were held on November 14, 1992 and June 19, 1993 to recommend experiments for running time at ATLAS. In FY 1993 the PhC members were Cary Davids (ANL), Teng Lek Khoo (ANL), Paul Kienle (GSI), Christopher J. Lister (Yale University), Robert McGrath (SUNY at Stony Brook), Walter E. Meyerhof (Stanford University), and Mark A. Riley (Florida State University). The PAC reviewed 26 proposals for 129 days of running time and 36 proposals for 172 days of running time at the meetings, respectively. The demand for running time at ATLAS continues to be more than double the time available on the accelerator .

The ATLAS User Executive Committee organized a User Group meeting during the October 1992 Division of Nuclear Physics APS meeting held in Santa Fe, New Mexico. The meeting was attended by approximately 35 scientists. The main topics of discussion were the FMA project, the status of ATLAS and APEX. In FY 1993 the ATLAS Executive Committee consisted of Stephen. Sanders (University of Kansas), Frank Wolfs (University of Rochester), Robert McGrath (SUNY at Stony Brook), and Christopher J. Lister (Yale University), as Chairperson.

The magnitude of the outside use of the accelerator during the past year has been substantial, as may be judged from the following two lists giving (1) the experiments performed by outside users and (2) the institutions represented. As may be seen from the names associated with each experiment, university groups are playing a major role in an important fraction of the experiments and a dominant role in some.

The Physics Division thanks the University of Chicago Board of Governors for their support of the ATLAS User Program. 


\section{a. Experiments Involving Outside Users}

All experiments in which outside users participated during FY 1993 are listed below. The spokesperson for each experiment is given in square brackets after the title. The names in parentheses are Argonne collaborators.

(1) Gamma Spectroscopy of ${ }^{183} \mathrm{Hg}$ and ${ }^{182} \mathrm{Hg}$ using the FMA [Bindra]

K. Bindra, A. Ramayya, W. C. Ma, B. Balabhadrapatruni, L. Chaturvedi,

J. Kormicki, Vanderbilt University; I. Bearden, Purdue University; W. Chung,

University of Notre Dame; (C. Davids, R. V. F. Janssens, T. Lauritsen, Y. Liang)

(2) Intermediate Structure in Elastic and Inelastic Channels of ${ }^{20} \mathrm{Ne}+{ }^{20} \mathrm{Ne}$ [Zurmühle]

R. Zurmühle, S. Barrow, J. Murgatroyd, N. Wimer, Y. Miao, K. Pohl, University

of Pennsylvania; (M. Freer, A. Wuosmaa)

(3) Anomalous Breakup of $56 \mathrm{Ni}$ [Sanders]

S. Sanders, K. Farrar, A. Hasan, F. Prosser, University of Kansas; I. Bearden,

B. Fornal, M. Sferrazza, Purdue University; C. Beck, CRN, Strasbourg; A. Szanto de Toledo, University of Sao Paulo; (R. V. F. Janssens, T. L. Khoo,

A. H. Wuosmaa, M. Freer, R. R. Betts, M. P. Carpenter, Y. Liang)

(4) Precision Spectroscopy of the $2 s-2 p$ Fine Structure Transition in He-Like Ar ${ }^{16+}$

[Livingston]

A. E. Livingston, F. Serpa, K. Kukla, University of Notre Dame; L. Curtis,

University of Toledo; (H. G. Berry, S. Cheng, R. W. Dunford, D. S. Gemmell,

J. Suleiman, C. Kurtz, E. P. Kanter)

(5) Deep-Inelastic Scattering at Energies Near the Coulomb Barrier [Gehring]

J. Gehring, University of Chicago; C. Jiang, Institute of Atomic Energy, Beijing;

K. Chan, Yale University; (M. Freer, D. Henderson, K. E. Rehm, J. P. Schiffer,

A. H. Wuosmaa, M. Wolanski)

(6) Development of AMS for Noble Gas Radioisotopes [Kutschera]

M. Paul, Hebrew University; (I. Ahmad, P. Billquist, B. G. Glagola, R. Harkewicz,

W. Kutschera, R. C. Pardo, K. E. Rehm)

(7) Fusion Cross Sections of $\mathrm{Ni}+\mathrm{Mo}$ [Rehm]

M. Paul, Hebrew University; J. Gehring,University of Chicago; (K. E. Rehm,

B. G. Glagola, D. Henderson, A. Wuosmaa)

(8) A Search for Shape Isomers in ${ }^{28} \mathrm{Si}$ [Freer]

K. Chan, Yale University; (M. Freer, A. H. Wuosmaa, R. R. Betts, D. Henderson)

(9) Mass and Decay of the $\mathrm{N}=\mathrm{Z}$ Nuclide ${ }^{68}$ Se [Davids]

K. Bindra, Vanderbilt University; I. Bearden, Purdue University; (Y. Liang,

C. N. Davids, R. V. F. Janssens, M. P. Carpenter, H. Penttila, T. Lauritsen)

(10) Test of Rotating Target

T.-F. Wang, Lawrence Livermore National Laboratory; (W. Kutschera, R. C. Pardo,

K. E. Rehm, J. P. Schiffer) 
(11) Transfer Reactions for ${ }^{92} \mathrm{Mo}+{ }^{58,64} \mathrm{Ni}$ [Rehm]

J. Gehring, University of Chicago; C. Jiang, Institute of Atomic Energy, Beijing;

(K. E. Rehm, B. G. Glagola, Y. Liang, W. Kutschera, A. H. Wuosmaa,

K. Teh)

(12) Lifetime Measurements with the DSAM Technique for Collective Excitations in ${ }^{195,196} \mathrm{~Pb}$ [Liang]

U. Garg, R. Mayer, University of Notre Dame; I. Bearden, B. Fornal, D. Nisius, Z. Grabowski, Purdue University; M. Drigert, Idaho National Engineering

Laboratory; (Y. Liang, R. V. F. Janssens, M. P. Carpenter, R. Henry, I. Ahmad, T. L. Khoo, T. Lauritsen)

(13) Search for Co-Existing Rotational Bands in ${ }^{187} \mathrm{Tl}$ [Reviol]

W. Reviol, L. Riedinger, J. Lewis, W. Mueller, B. Zimmerman, University of

Tennessee; (I. Ahmad, T. L. Khoo, M. P. Carpenter, R. V. F. Janssens,

T. Lauritsen, Y. Liang, R. Henry)

APEX Beam Tests [Betts]

T. Happ, GSI; S. Austin, E. Kashy, J. Winfield, J. Yurkon, Michigan State

University; A. Perrera, F. Wolfs, University of Rochester; S. Gazes, University of

Chicago; J. Greenberg, K. Chan, N. Kaloskamis, C. Lister, A. Chishti, Yale

University; A. Hallin, M. Liu, Queens University; T. Trainor, University of

Washington; J. Fox, E. Roa, Florida State University; (R. R. Betts,

A. H. Wuosmaa, W. Kutschera, B. B. Back, J. P. Schiffer, I. Ahmad,

D. Henderson, R. W. Dunford, M. Freer)

(15) Accelerator Mass Spectrometry of $210,000-\mathrm{yr}^{81} \mathrm{Kr}$ [Kutschera]

M. Paul, Hebrew University; (W. Kutschera, I. Ahmad, P. Billquist,

B. G. Glagola, R. Harkewicz, R. C. Pardo, K. E. Rehm)

(16) He-Like Argon Speciroscopy [Livingston]

A. E. Livingston, K. Kukla, University of Notre Dame; L. Curtis, University of

Toledo; (H. G. Berry, R. W. Dunford, E. P. Kanter, D. S. Gemmell,

J. Suleiman, M. Talebian)

Development of an Implantation Facility at the FMA Focal Plane [Davids]

K. Bindra, A. Ramayya, B. Balabhadrapatruni, Vanderbilt University;

B. Zimmerman, C. Bingham, University of Tennessee; W. B. Walters, University of Maryland;

(C. N. Davids, M. Freer, H. Penttila, K. E. Rehm, Y. Liang, T. Lauritsen,

D. J. Henderson, R. Henry, A. Wuosmaa)

RDM Lifetime Measurements in ${ }^{182} \mathrm{Pt}$ [Garg]

U. Garg, J. Walpe, S. Nagulswaran, Q. Ren, B. Davis, University of Notre Dame;

I. Bearden, Purdue University; W. Reviol, X.-Z. Wang, University of Tennessee;

(I. Ahmad, T. Lauritsen, M. P. Carpenter, R. V. F. Janssens,

R. Henry, T. L. Khoo)

(19) Lifetime Measurements in ${ }^{181}$ Ir and ${ }^{187} \mathrm{Au}$ [Garg]

U. Garg, J. Walpe, S. Nagulswaran, Q. Ren, B. Davis, University of Notre Dame;

I. Bearden, Purdue University; W. Reviol, X.-Z. Wang, University of Tennessee;

(I. Ahmad, T. Lauritsen, M. P. Carpenter, R. V. F. Janssens,

R. Henry, T. L. Khoo) 
(20) Precision Spectroscopy of the $2 s-2 p$ Fine Structure Transitions in He-Like Ni $26+$ [Livingston]

A. E. Livingston, K. Kukla, University of Notre Dame; (R. W. Dunford,

D. S. Gemmell, S. Cheng, C. Kurtz, B. Zabransky, M. Talebian)

(21) Spectroscopy of Very Neutron Rich Deformed Pb Nuclei [Baxter]

A. Baxter, A. Byrne, G. Dracoulis, Australian National University; I. Bearden,

D. Nisius, Purdue University; (M. P. Carpenter, R. Henry, C. N. Davids,

H. Penttila, T. L. Khoo, R. V. F. Janssens, T. Lauritsen)

(22) Study of Nuclear Structure Effects in the Mo + Mo System [Rehm]

J. Gehring, University of Chicago; C. Jiang, Institute of Atomic Energy, Beijing;

K. Chan, Yale University; (K. E. Rehm, B. G. Glagola, Y. Liang,

W. Kutschera, A. H. Wuosmaa, K. Teh)

(23) Deformation in the $\mathrm{N}<126, \mathrm{Z}>82$ Region [Freeman]

S. Freeman, University of Manchester; P. Chowdhury, D. Blumenthal, C. Lister, Yale University; D. Nisius, Purdue University; (M. P. Carpenter, R. Henry,

T. L. Khoo T. Lauritsen, R. V. F. Janssens, C. N. Davids, H. Penttila,

D. Henderson)

(24) The Alpha-Eecay Rates of ${ }^{180} \mathrm{~Pb}$ and ${ }^{182} \mathrm{~Pb}$ [Toth]

K. Toth, R. Auble, C. Gross, P. Mantica, Oak Ridge National Laboratory;

C. Bingham, B. Zimmerman, J. Richards, University of Tennessee;

L. Conticchio, University of Maryland; P. Joshi, Louisiana State University:

D. Moltz, Lawrence Berkeley Laboratory; (C. N. Davids, H. Penttila,

D. Henderson)

25) Crystal Diffraction Spectroscopy of He-Like Ca: A Timely Application of ATLAS AccelDecel [Deslattes]

R. D. Deslattes, J. Schweppe, C. Chantler, L. Hudson, National Institute of

Standards and Technolggy; (R. W. Dunford, H. G. Berry, P. Cowan,

E. P. Kanter, C. Kurtz, S. Cheng, J. Suleiman)

(26) FMA Studies of Proton-Rich Nuc..: $\mathrm{N} \sim 82$ by Gamma and Conversion Electron Spectroscopy [Grabowski]

Z. Grabowski, D. Nisius, I. Bearden, R. Mayer, P. Daly, M. Sferrazza,

L. Richter, Purdue University; (R. V. F. Janssens, B. Crowell, I. Ahmad,

C. N. Davids, H. Penttila, T. Lauritsen)

(27) n-n and n-d Correlations [DeYoung]

P. DeYoung, T. Butler, C. Dykstra, Hope College; G. Gilfoyle, M. Nimchek,

A. Snyder, University of Richmond; R. Kryger, Michigan State University;

J. Hinnefeld, Indiana University at South Bend; J. J. Kolata, M. Belbot,

W. Chung, K. Lamkin, University of Notre Dame; M. Kaplan, Carnegie-Mellon University

(28) Gamma-Ray Studies of N-Rich Products of Deep-Inelastic ${ }^{124} \mathrm{Sn}+{ }^{136} \mathrm{Xe}$ Collisions [Fornal]

B. Fornal, R. Mayer, I. Bearden, D. Nisius, L. Richter, M. Sferrazza, R. Broda,

Z. Grabowski, P. Daly, Purdue University; (M. P. Carpenter, R. Henry,

B. Crowell, R. V. F. Janssens, T. L. Khoo, T. Lauritsen) 
(29) Test of APEX Heavy-Ion Counters

T. Happ, GSI; S. Austin, E. Kashy, J. Winfield, J. Yurkon, Michigan State University; A. Perrera, F. Wolfs, University of Rochester; S. Gazes, University of Chicago; J. Greenberg, K. Chan, N. Kaloskamis, C. Lister, A. Chishti, Yale University; A. Hallin, M. Liu, Queens University; T. Trainor, University of Washington; J. Fox, E. Roa, Florida State University; (R. R. Betts, A. H. Wuosmaa, W. Kutschera, B. B. Back, J. P. Schiffer, I. Ahmad, D. Henderson, R. W. Dunford, M. Freer)

(30) Heavy Ion Irradiations of High-Temperature Superconductors [Hettinger]

J. Hettinger, D. Miller, K. Gray, Materials Science Division, ANL

(31) An Atomic Clock for Fission [Sobotka]

L. Sobotka, D. Robertson, R. Charity, D. Sarantites, Washington University

(32) Search for Low-Spin Shape Isomerism in 66 Ni [Sanders]

S. Sanders, F. Prosser, K. Farrar, A. Dummer, C. Schmeisner, University of Kansas; B. Fornal, M. Sferrazza, R. Mayer, D. Nisius, Purdue University; C. Beck, F. Hass, D. Maboub, CRN, Strasbourg; S. Cavallero, University of Catania; (M. P. Carpenter,

T. L. Khoo)

APEX - Fositron Preduction in Heavy Ion Collisions [Betts]

T. Happ, GSI; S. Austin, E. Kashy, J. Winfield, J. Yurkon, Michigan State University; A. Perrera, F. Wolfs, University of P.ochester; S. Gazes, University of Chicago; J. Greenberg, K. Chan, N. Kaloskamis, C. Lister, A. Chishti, Yale University; A. Hallin, M. Liu, Queens Unt versity; T. Trainor, University of Washington; J. Fox, E. Roa, Florida State University; (R. R. Betts, A. H. Wuosmaa, W. Kutschera, B. B. Back, J. P. Schiffer, I. Ahmad, D. Henderson, R. W. Dunford, M. Freer)

(34) Excitation Function for the ${ }^{138} \mathrm{Ba}\left({ }^{48} \mathrm{Ca}, \mathrm{xn}\right)$ Reaction [Carpenter]

L. Nisius, Purdue University; (M.P. Carpenter, B. Crowell, I. Ahmad, R. Henry,

T. L. Khoo, T. Lauritsen)

Alignment Check of the BGO Array

I. Bearden, Purdue University; (R. V. F. Janssens, M. P. Carpenter, T.

Lauritsen) 


\section{b. Outside Users of ATLAS and of ATLAS Technology During the Period October 1, 1992 - September 30, 1993}

This list includes only those who were present at ATLAS for an experiment or other related research. An asterisk denotes students.

(1) University of Kansas

* A. Dummer

* K. Fairar

* A. Hasan

F. Prosser

* $\quad$ C. Sanders

(2) University of Notre Dame

* M. Belbot

* W. Chung

* B. Davis

U. Garg

J. Kolata

* K. Kukla

* K. Lamcin

A. E. Livingston

* S. Naguleswaran

* Q. Ren

* F. Serpa

* R. Thompson

* R. Tiohe

* J. Walpe

(3) Purdue University
* I. Bearden
R. Broda
P. Daly
B. Fornal
Z. Grabowski
* R. Mayer
* D. Nisius
* L. Richter
M. Sferrezza

(4) Idaho National Engineering Lab M. Drigert

(5) Vanderbilt University

B. Balabhadrapatruni

K. Bindra

L. Chaturvedi

J. Kormicki

W.-C. Ma

A. Ramayya
(6)

\begin{tabular}{ll}
\multicolumn{3}{l}{ University of Pennsylvania } \\
$*$ & S. Barrow \\
$*$ & Y. Miao \\
& J. Murgatroyd \\
$*$ & K. Pohl \\
& N. Wimer \\
& R. Zurmühle
\end{tabular}

(7)

Washington University

R. Charity

D. Robertson

D. Sarantites

L. Sobotka

(8) Lawrence Livermore National Lab T.-F. Wang

(9) University of Rochester

S. Gazes

* A. Perrera

(9) University of Rochester (Contd.)

F. Wolfs

(10) Carnegie-Mellon University

M. Kaplan

(11) University of Chicago

S. Gazes

* J. Gehring

(12) University of Catania

S. Cavallero

(13) University of Toledo

L. Curtis

(14) NSC, New Delhi

N. Anantaraman

G. Mehta

P. Potukuchi

A. Roy

(15)

Hope College

* T. Butler

* P. DeYoung

* C. Dykstra 
(16) Louisiana State University
E. Zganjar
P. Joshi

(17) Institute of Atomic Energy, Beijing

C. Jiang

(18) CRN, Strasbourg
C. Beck
F. Hass
D. Maboub

(19) Australian National University
A. Baxter
A. Byrne
G. Dracoulis

(20) University of Maryland

W. Walters

L. Conticcio

(21) Yale University

* D. Blumenthal

* K. Chan

A. Chishti

P. Chowdhury

* B. Crowell

J. Greenberg

* N. Kaloskamis

C. Lister

(22) Materials Science Division, ANL
K. Gray
J. Hettinger
D. Miller

(23) Hebrew University

M. Paul

(24) University of Tennessee

C. Bingham

* J. Lewis

W. Mueller

L. Riedinger

W. Reviol

X.-Z. Wang

B. Zimmerman

(25) University of Wasnington

T. Trainor

(26) Florida State University
J. Fox
E. Roa

(27)

$$
\begin{aligned}
& \text { University of Sao Paulo } \\
& \text { J. C. Acquadro } \\
& \text { N. Added } \\
& \text { M. Ferraretto } \\
& \text { J. Ordonez } \\
& \text { O. Sala } \\
& \text { A. Szanto de Toledo }
\end{aligned}
$$

Michigan State University

S. Austin

E. Kashy

R. Kryger

D. Mercer

J. Winfield

J. Yurkon

(29)

Princeton University

F. Calaprice

(30) National Inst. of Stds. \& Tech.
C. Chantler
R. Deslattes
L. Hudson
J. Schweppe

(31) Oak Ridge National Laboratory

R. Auble

C. Gross

P. Mantica

K. Toth

(32) University of Richmond

$$
\begin{array}{ll} 
& \text { G. Gilfoyle } \\
* & \text { M. Nimchek } \\
* & \text { A. Snyder }
\end{array}
$$

(33) Lawrence Berkeley Laboratory

D. Moltz

(34) Indiana State Univ. at South Bend J. Hinnefeld

(35) University of Manchester

S. Freeman

W. Phillips

(36) GSI, Darmstadt

T. Happ

Queens University, Ontario
A. Hallin

M. Liu 


\section{c. Summaries of the Continuing User Programs for FY 1993 \\ c.a. The University of Notre Dame}

c.a.1. Nuclear Physics (U. Garg, S. Dixit, J. J. Kolata, W. Reviol (University of Tennessee), A. Aprahamian, B. Davis, S. Naguleswaran, J. Wa'pe)

Several groups from the University of Notre Dame are playing an important role in developing the research program at ATLAS. One of their main interests is the study, in collaboration with ANL staff members, of the behavior of nuclei at high spin in the transitional region near ${ }^{208} \mathrm{~Pb}$ (i.e. the $\mathrm{Hg}-\mathrm{Pt}-\mathrm{Os}$ nuclei), and $\mathrm{Sn}$ (i.e. the $\mathrm{Ru}-\mathrm{Pd}$ nuclei) with emphasis on shape coexistence and configuration mixing. This group has also participated in many experiments performed with the BGO gamma-ray facility. A major activity of this past year was the continued maintenance and development of the gamma-ray facility consisting of a BGO sum-multiplicity array of 50 elements combined with 12 Compton-suppressed germanium detectors. In this project, the Notre Dame gruup is responsible for the array. In addition, part of the Notre Dame group has built and tested a Bragg-curve detector for use in the focal plane of the Fragment Mass Analyzer and a plunger for lifetime measurements with the $\gamma$-ray facility.

\section{c.a.2. Atomic Physics (A. E. Livingston)}

In a collaboration with the Atomic Physics group at Argonne, measurements are being made of the fine structure in lithium-like and helium-like ions using beam-foil spectroscopy. The current goal is to measure the $2^{3} \mathrm{~S}_{1}-2^{3} \mathrm{P}_{0}$ transition energies in helium-like $\mathrm{Ni}^{26+}$ and $\mathrm{Ar}^{16+}$. Data analysis has been completed on the spectroscopy of $\mathrm{Ar}^{16+}$. Precise measurements of $2 \mathrm{~s}-2 \mathrm{p}$ transition energies in simple (few-electron) atomic systems provide stringent tests of several classes of current atomicstructure calculations. Another collaboration between Argonne and E. Träbert of Ruhr Universität at Bochum measured the lifetimes of intercombination transitions in $\mathrm{Mg}$-like, Al-like and Si-like bromine. The group is also participating in measurements of the forbidden transitions in heliumlike ions. The excited state is formed in a thin-carbon foil which can be moved relative to the $\mathrm{Si}(\mathrm{Li})$ detector by means of a precision translator. The decay rate is measured as a function of foildetector distance to determine the lifetime. In the past year data analysis was completed for a measurement of the lifetime of the ${ }^{1} \mathrm{~S}_{0}$ level in helium-like bromine. A measurement of the lifetime of the $2^{3} \mathrm{P}_{2}$ level in helium-like krypton is planned for this year.

\section{c.b. Purdue University (P. Daly, Z. Grabowski, R. H. Mayer, D. Nisius, B. Fornal, I. Bearden)}

The Purc'se University group, including several thesis students, is working on a measurement of high-spin nuclear states at ATLAS. They use in-beam gamma-ray techniques to investigate several aspects of nuclear structure at high spin, testing the validity of shell-model calculations for highspin-yrast states near $Z=50$. The nuclei are produced via deep inelastic reactions, rather than with the more conventional fusion reactions. This technique allows the study of neutron-rich nuclei that cannot be studied by other means. The group will also be studying proton-rich nuclei with $\mathrm{N} \sim 82$ using the FMA and an electron spectrometer. 
c.c. University of Kansas (S. Sanders, F. W. Prosser, K. Farrar, A. Hasan, A. Dummer, and C. Schmeisner)

This past year the Ka: sas group has continued its studies of the fusion-fission process in light nuclear systems and has started a search for a possible low-spin superdeformed configuration in the ${ }^{66} \mathrm{Ni}$ nucleus. The emphasis of the fusion-fission program has been to determine the role of nuclear structure at the saddle-point configuration on the population of states in the fission fragments. In an analysis completed this year of an ATLAS particle-particle- $\gamma$ coincidence experiment for the ${ }^{32} \mathrm{~S}+{ }^{24} \mathrm{Mg}$ reaction, they have found evidence for an enhanced population of a strongly prolate deformed band in the ${ }^{28} \mathrm{Si}$ symmetric-mass fission channel. For this analysis the group has developed a model calculation, based on the fission transition-state model, that uses the known level structure of the fission fragments to predict the population of specific mutual excitations of these fragments. This statistical-model calculation helps in distinguishing those features of the data that can be accounted for by a spin-weighted population of the fragment states from those that arise from details of the nuclear structure. In a related investigation, this group has been developing the relationship between the fusion-fission process in light systems and the heavyion resonance mechanism that is also found in some of these systems. This work is focused on the ${ }^{48} \mathrm{Cr}$ compound system, which can be reached by a number of different entrance channels using the ATLAS facility. The group has so far obtained particle-particle coincidence data for the ${ }^{24} \mathrm{Mg}+{ }^{24} \mathrm{Mg}$ reaction, where strong resonance behavior has be ?n observed in the past, and they have also obtained particle-particle- $\gamma$ coincidence data for the ${ }^{36} \mathrm{Ar}+12 \mathrm{C}$ reaction, a very asymmetric entrance channel reaching the ${ }^{48} \mathrm{Cr}$ compound nucleus. The ${ }^{24} \mathrm{Mg}+{ }^{24} \mathrm{Mg}$ data have been analyzed using the statistical model with the conclusion that both the fusion-fission process and the resonance mechanism are significant in the ${ }^{24} \mathrm{Mg}$ symmetric-mass breakup channel, with fission dominating at higher excitation energies. This work has led to a Ph.D. being granted to Kansas graduate student, A. Hasan. The ${ }^{36} \mathrm{Ar}+{ }^{12} \mathrm{C}$ data are still being analyzed, but significant differences in the ${ }^{24} \mathrm{Mg}$ excitation-energy spectra for the two entrance channels are already evident. As a new initiative, the group has started to explore the possibility of low-spin, superdeformed configurations in light systems. States corresponding to these configurations would presumably decay through $\gamma$-ray emission rather than through the fission mechanism. In a preliminary experiment at ATLAS last summer, the group explored the production rates for the $66 \mathrm{Ni}$ nucleus, where a low-spin superdeformation has been predicted, using the ${ }^{136} \mathrm{Xe}+{ }^{64} \mathrm{Ni}$ reaction. The measurement, which used a particle-particle- $\gamma$ coincidence arrangement at the $\gamma$-ray facility, went very well and is currently being analyzed.

Papers prepared on the ${ }^{32} \mathrm{~S}+{ }^{24} \mathrm{Mg}$ and ${ }^{24} \mathrm{Mg}+{ }^{24} \mathrm{Mg}$ analyses have been accepted for publication. The ${ }^{36} \mathrm{Ar}+{ }^{12} \mathrm{C}$ experiment involved a collaboration with representatives from Argonne, Cracow, Kansas, Strasbourg, and São Paulo. The ${ }^{136} \mathrm{Xe}+{ }^{64} \mathrm{Ni}$ experiment was done by a collaboration including researchers from Argonne, Catania, Cracow, Kansas, Purdue, and Strasbourg.

c.d. National Institute of Standards and Technology (R. D. Deslattes, P. Indelicato, and E. Kessler, Jr.)

A program is in progress to carry out accurate spectroscopic measurements of $\mathrm{X}$-ray transitions in hydrogen-like and helium-like calcium to provide important tests of QED and relativistic quantum mechanics calculations. In order to produce clean spectral lines in the experiment, a gas target is being used to obtain the excited helium-like or hydrogen-like ions. To get reasonable cross sections for electron pickup, the technique of accel/decel is employed, whereby beams of oneelectron ions are obtained by stripping after the booster and then slowing down in the ATLAS section of the linac. Data analysis is in progress fora measurement of the $2 \mathrm{p}-2 \mathrm{~s}$ transition energies 
in He-like calcium. A beam of $\mathrm{Ca}^{19+}$ was accelerated to $205 \mathrm{MeV}$ and then decelerated to 105 $\mathrm{MeV}$ and delivered to the atomic-physics beam line. A crystal spectrometer and a gas target installed on the beam line were used to make precision X-ray measurements. Plans are in progress for making improvements to the beam diagnostics, gas cell, and X-ray spectrometer.

\section{c.e. Idaho National Engineering Laboratory (M. W. Drigert)}

This effort has been associated with research programs done with the Argonne-Notre Dame BGO $\gamma$-ray facility. Within the collaboration it involves the responsibility for the maintenance and continuous upgrade of the software used to analyze the data taken with the facility. Among the present tasks under way are the migration of the analysis software to UNIX platforms and the expansion of the software's graphics capabilities. The physics questions being addressed are superdeformation studies in the Dy and $\mathrm{Pb}$ mass regions, and the study of octupcle stability in the light actinide mass region. Future research plans include further studies of nuclei in the light actinide region, with the emphasis on using the FMA in conjunction with the Ge detectors from the $\gamma$-ray facility to investigate nuclei which can only be populated by charged particle evaporation channels.

c.f. Vanderbilt University (A.V. Ramayya, J. H. Hamilton, K. Bindra, W.-C. Ma, B. R. S. Babu, L. Chaturvedi, and J. Kormicki)

An experiment identifying gamma-ray transitions in ${ }^{183} \mathrm{Hg}$ using $\gamma-\gamma$ and recoil- $\gamma$ coincidences has been performed at the FMA and published. Five bands were observed, of which two are associated with the [624]9/2+ orbital and exhibit signature splitting. Two other bands which do not show signature splitting have been associated with the [514]7/2- orbital and exhibit transition energies almost identical to bands with the same configuration in ${ }^{185} \mathrm{Hg}$. New experiments on identification of bands in ${ }^{181} \mathrm{Hg}$ and an in-beam study of conversion electrons from ${ }^{183} \mathrm{Hg}$ will be performed later in 1997.

c.g. University of Tennessee, University of Maryland, and Oak Ridge National

Laboratory (C. R. Bingham, J. D. Richards, B. Zimmerman, W. B. Walters,

L. F. Conticchio, and K.S. Toth)

This collaboration has ber: $\mathrm{n}$ involved in the study of alpha-decaying nuclei in the $\mathrm{A}>180$ region using the double-sided silicon strip detector implantation facility at the FMA. They are also active participants in the proton radioactivity experiments (see Sec. I.A.c.8.). An experiment on highspin isomers in the neutron-deficient $\mathrm{Cd}$ region is planned for the near future.

\section{d. ATLAS - Technology Transfer}

In addition to outside users of the ATLAS beam, we also provide assistance in the use of ATLAS technology at other laboratories.

\section{d.a. Florida State University (J. Fox, A. Frawley, and E. Myers)}

Argorne fabricated the niobium resonators and some auxiliary devices for the superconductinglinac energy booster built at Florida State University. Personnel from FSU came to ANL to assemble and test the resonators. The main resonator fabrication work for FSU was completed during 1986, but we continue to interact with personnelconcerning ongoing refinements in the technology. Topics in which we were most recently involved are (1) a change in the method of cooling the FSU resonators and (2) the transfer of information about fast tuner upgrades. 


\section{d.b. Kansas State University (T. Gray, K. Karnes, and V. Needham)}

Argonne has fabricated the niobium resonators and some other linac components required for the superconducting accel/decel linac now in operation at Kansas State University. Several staff members from KSU spent a substantial period of time at ANL during FY 1985 in order to learn the technology, and they return occasionally to assemble and test the resonators. There is a continuing interchange of technical information between ANL and KSU related to linac operations, tuning, and resonator maintenance.

d.c. University of Sao Paulo (J.C. A.cquadro, N. Added, M. Ferraretto, J. Ordonez, A. Szanto de Toledo)

Argonne has agreed to assist the University of Sao Paulo (USP) in the construction of a small superconducting heavy-ion linac to serve as an energy booster for projectiles from their 8-MV tandem. This booster will be similar in many respects to the ANL booster linac built in the late 1970s. The ANL contribution to this project will be (1) to build (at USP expense) 14 split-ring niobium resonators and some of the associated rf electronics, (2) to provide technical information, and (3) to train USP staff members in several phases of superconducting-linac technology. Two Brazilian engineers worked at Argonne for one year, gaining experience in cryogenics and in superconducting-resonator technology. Another engineer worked on the new control system at ATLAS for two years, the first year supported by Sao Paulo and the second with direct ANL support. Sao Paulo personnel returned to ANL in 1993 for assembly and testing of the first batch of completed resonators. The fabrication of the resonators will be completed by early 1994 when the Sao Paulo personnel will come back for more assembly and testing.

\section{d.d. Nuclear Science Centre, New Delhi (G. Mehta, P. Potukuchi, A. Roy)}

Argonne is collaborating with the Nuclear Science Centre (NSC), New Delhi, to develop a new type of superconducting accelerating structure for low-velocity heavy ions. A copper model has been evaluated and the niobium prototype is currently nearing completion. All funding for the prototype has come from the NSC, and they have also stationed two staff members at ATLAS for the past two years to gain experience ano work on this project. Additional NSC personnel will visit ATLAS for extended periods during 1994 for electronics and cryogenics experience and training. 


\section{OPERATION AND DEVELOPMENT OF TTLAS}

These sections report on the operation and development of the Argonne Tandem Linear Accelerator System (ATLAS) as a national user facility, for basic research in nuclear physics. The facility is also used for atomic physics and occasionally for other areas of research, such as materials science. Over half of the beam time is allocated to outside users. A positive-ion injector (PII) was completed and commissioned in FY 1992, significantly upgrading the capabilities of ATLAS, and thereby also creating additional demands for beam tirne. Including the new PII linac, the complete ATLAS accelerator now consists of 50 independently-controlled superconducting accelerating structures. Beams with masses up to uranium are now routinely available for research. Figure II- 1 shows the distribution of beams delivered in FY1993. Currently, the facility is on a seven-day week operating schedule, made possible with incremental funds in FY1994.

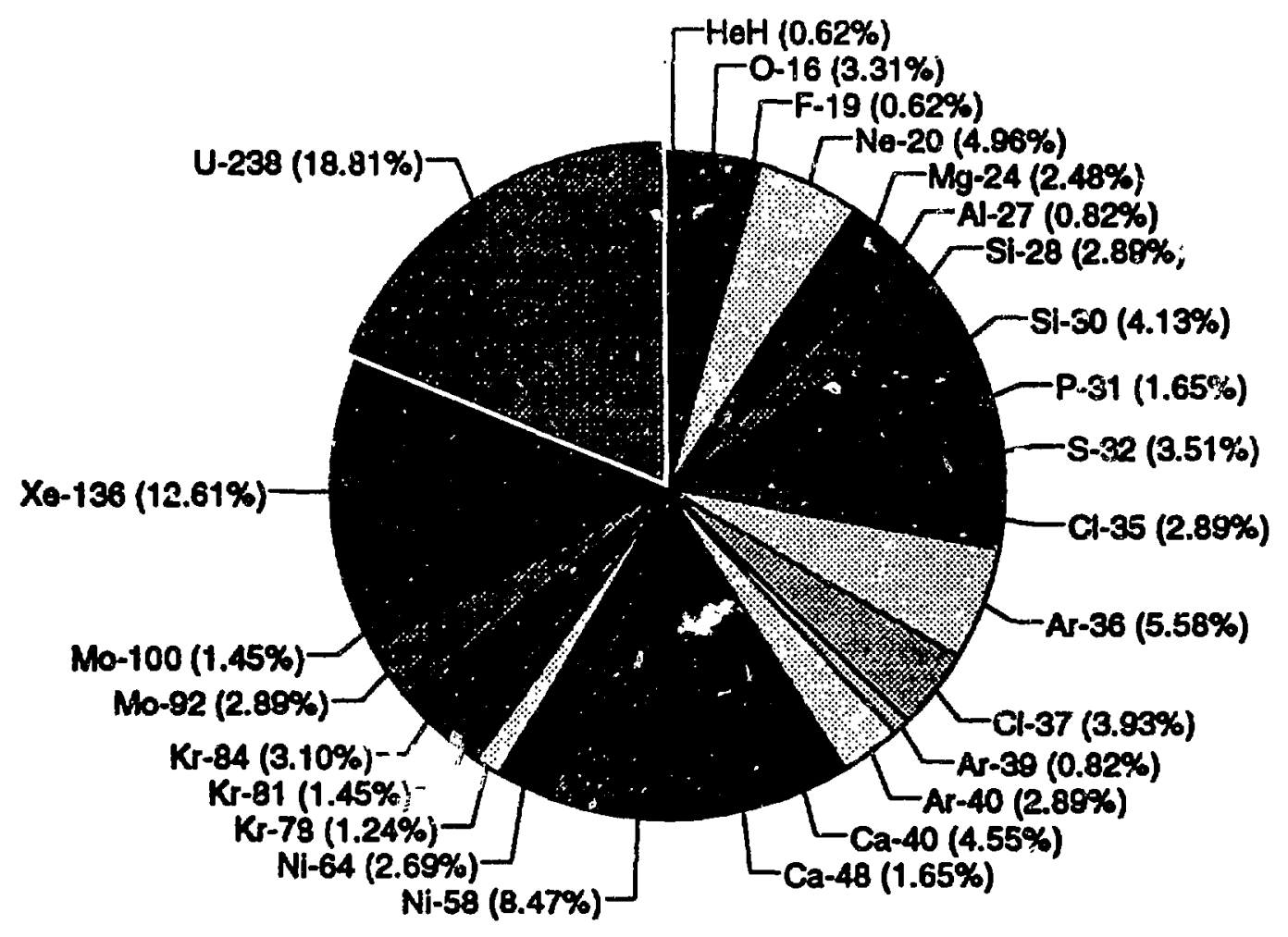

Fig. II-1. "PII" chart of the beams used in research at ATLAS during FY1993, the first full year in which the new positive-ion injector was used.

These activities are elaborated on in the sections below. There are progress reports on recent beam developments, as well as on other technical developments at ATLAS, and descriptions of superconducting $R F$ and other accelerator physics $R \& D$ projects. 
ATLAS EXPERRIENT SUMMARY

\begin{tabular}{|c|c|c|c|c|}
\hline & $\frac{\text { FY } 1993}{\text { (actual) }}$ & $\frac{\text { FY } 1994}{(}$ & $\frac{\text { FY } 1995}{\text { predicted }}$ & FY 1996 \\
\hline \multicolumn{5}{|l|}{ Beam Use for Research (hr) } \\
\hline Nuclear Physics & 2867 & 4050 & 4550 & 4750 \\
\hline Atomis Physics & 282 & 350 & 350 & 350 \\
\hline \multirow[t]{2}{*}{ Other } & 75 & 100 & 100 & 100 \\
\hline & $\overline{3224}$ & $\overline{4500}$ & $\overline{5000}$ & $\overline{5200}$ \\
\hline $\begin{array}{l}\text { Number of Nuclear } \\
\text { Experiments Receiving Beam }\end{array}$ & 47 & 50 & 60 & 60 \\
\hline $\begin{array}{l}\text { Number of Scientists } \\
\text { Participating in Research }\end{array}$ & 147 & 150 & 150 & 150 \\
\hline \multicolumn{5}{|l|}{ Institutions Represented } \\
\hline Universities (U.S.A.) & 23 & 29 & 29 & 29 \\
\hline DOE National Laboratories & 6 & 5 & 5 & 5 \\
\hline Other & 14 & 12 & 12 & 12 \\
\hline \multicolumn{5}{|l|}{ Usage of Beam Time (\%) } \\
\hline In-House Staff & 29 & 42 & 42 & 42 \\
\hline \multirow{2}{*}{ Universities (U.S.A.) } & 61 & 53 & 53 & 53 \\
\hline & 4 & 2 & 2 & 2 \\
\hline $\begin{array}{l}\text { Other DOE National Laboratories } \\
\text { Other Institutions }\end{array}$ & $\frac{6}{100}$ & 3 & $\frac{3}{3}$ & 3 \\
\hline Totai & 100 & 100 & 100 & 100 \\
\hline
\end{tabular}

\section{A. OPERATION OF THE ACCELERATOR}

R. C. Pardo, B. Batzka, P. J. Billquist, J. Bogaty, B. E. Clifft, S. L. Craig, R. E. Harden, R. Harkewicz, D. Herbst, B. Millar, F. H. Munson, Jr., K. Nakagawa, D. R. Phillips, J. R. Specht, P. Strickhorn, A. Sutherland, B. Tieman, I. R. Tilbrook, R. Vondrasek, G. Wiemerslage, and G. P. Zinkann

Highlights of ATLAS operation in FY1993 were the continued improvement in our ability to deliver the heaviest of beams, ${ }^{238} \mathrm{U}$, reliably and with good beam intensity and the increase in our operator staff so that ATLAS could achieve a seven-day-per-week operating schedule at the end of the year. Technological improvements contributing significantly to improved performance for uranium and other beams included an increase of approximately 2 units in the average charge state of beams from the ECR ion source resulting from the use of an aluminum extractor electrode and first-stage face components in the source and the addition of a wet engine to one of the cryogenic refrigerators. Work continued in FY 1993 on a new master oscillstor which adds the ability to control the relative phase of each major component of the accelerator with respect to each other. This feature is expected to significantly improve the ability to use saved parameters from previous tunes and scale values from those parameters. The new master oscillator was installed in December 1993 and immediately showed its usefulness in a 'guide beam' setup for a lead run in January 1994.

Operation of the ATLAS accelerator with the new Positive-Ion Injector has continued to increase. For FY 1993 approximately $62 \%$ of all beam time was provided with the PII, reflecting continued growth in the demand for heavier beams as well as the availability of some medium-mass beams which the tandem injector is unable to provide with sufficient intensity such as molybdenum, 
calcium, and the noble gases. The tandem continues to perform in a highly reliable fashion and is still an important component of the ATLA; facility when beams lighter than mass $A \leq 70$ and moderate intensity is sufficient. The tandem is in need of some preventive maintenance, especially on the voltage control system (corona system). This is identified in the capital equipment section.

The ATLAS facility delivered a total of 26 different ion species to the experimental program during FY 1993. Beam masses ranged from $A=5\left(\mathrm{HeH}^{+1}\right)$ to $A=238\left({ }^{238} \mathrm{U}^{26,39+}\right)$. A strong emphasis is the demand for the heavier mass beams. This year beams with $\mathrm{A}>100$ constituted more than one-third of our running time. Such an emphasis on the heaviest beams requires the facility to operate near its accelerating limit much of the time and that produces some loss due to unscheduled down-tim:, especially resonator conditioning requirements. This effect plus the approximately 150 hours of lost time from the lost power incident of May 11, 1993, explain much of the worsened 'unscheduled maintenance' item in the 'Accelerator Operations Summary' table. We helieve that we are making progress in improving this situation with work in a number of areas silch as improved cryogenic capacity, conditioning techniques and training, as well as simply more frequent operation at these high performance levels.

Operation of the ATLAS system has been reliable with a typical experiment receiving beam over $90 \%$ of its scheduled hours. The most serious lost time occurred on May 11 when a cable providing power to our building shorted interrupting power to the entire central region of Argonne for over two hours - including ATLAS. Cryogenic systems do not react favorably to such insults and the remainder of the week was spent in recovering, recooling, and refilling helium into the accelerator. By Friday, May 14, we were able to again accelerate beam and the scheduled APEX experiment then continued shortly thereafter through the weekend and the following week.

The continued development of a uranium beam for the APEX experiment has been the primary focus of activity for the accelerator staff. In the period from February to June, three distinct periods of two weeks each were scheduled for the APEX experiment. During that time the performance of the accelerator continued to improve due to improvements in the velocity profile of the linac, improved field performance of the resonators, and some improvements in source operation. These improvements resulted in a May run which achieved a maximum ${ }^{238} \mathrm{U}$ energy of $6.45 \mathrm{MeV} / \mathrm{A}(1.35 \mathrm{GeV})$. The PII resonaiors performed at record levels - averaging 3.6 $\mathrm{MV} / \mathrm{m}$ accelerating gradient in a highly reliable nuanner. With the improved resonator performance, the charge state from the ECR source was reduced to $24^{+}$and the charge state from the beam stripping at the end of the PII was reduced to $39^{+}$. This resulted in a delivered beam current of up to 5 pnA on target during the May run. More typical current on target was 3 pnA.

ATLAS was down for a major maintenance period which started the week of Labor Day and lasted approximately six weeks. The most important project accomplished during that period was the installation of a new wet engine at the ATLAS helium refrigerator. This device replaces a Joule-Thompson expansion valve and extracts additional energy out of the helium resulting in improved cryogenic efficiency and an increase in total refrigeration capacity of about 50 watts. The wet engine also reduces the average electrical power consumption due to the improved refrigeration efficiency.

In addition to the wet engine installation, two additional 'V-series' resonators in ATLAS were replaced with 'H-series' resonators. The resonator exchange continues the velocity upgrade of the linac and provides ATLAS with a more optimized velocity profile for the very heavy ions which we are now able to provide to the experimental program.

After the September-October 1993 shutdown for these system improvements, another two-week uranium APEX run occurred. The performance was similar to the May run but total hours with beam on-target was significantly greater and the system ran more reliably. The delivered beam 
current was typically 2-3 pnA. The most serious accelerator problem limiting beam delivery was the quenching of solenoids in the 'booster section' of the linac where the unwanted charge states from the stripping process are discarded. The beam power in these lost beam components is sufficient to cause some of the solenoids to go normal and quench. This problem will be eliminated with the installation of the charge-state selector after the PII linac in the summer of 1994.

\section{B. RECENT AND PLANNED IMPROVEMENTS AT ATLAS}

\section{a. Upgrade of the Linac Control System (F. H. Munson Jr., and M. Ferraretto)}

The ATLAS beam lines are now fully controlled by the new Linac Control System. A beam-line database is now in place and simple focal-length scaling routines are in place. Controi of solenoids in the linac is the next priority for the development group and that task is nearing completion. Cryogenic system parameters monitoring was implemented last year and monitoring of resonator and VCX temineratures is nearing the implementation stage.

The old control system continues to operate in parallel with the new system and provides control and monitor functions for the linac system. Many of these functions will migrate to the new control system during this next year.

M. Ferraretto returned from Brazil for approximately three months this year and provided important assistance in the development of the system. This Argonne-Sao Paulo collaboration provided important benefits for us in this project. Ferraretto will continue to consult and work on the project via the computer network from Brazil during this year.

\section{b. New Master Oscillator System (B. E. Clifft, R. C. Pardo, and P. Strickhorn)}

A new master oscillator for ATLAS was developed during FY 1993 and was installed during the 1993 Christmas shutdown. ATLAS is now operating with its new heart and we have already seen some of the expected benefits of it new features. The master oscillator upgrade makes ATLAS a much more flexible system and is expected to decrease the time required to switch between beams. The most important feature of the new master oscillator is the ability to adjust the relative operating phase of major sections of the linac (PII, Tandem buncher, Booster, ATLAS) with respect to each other. This new feature adds a tunable parameter for matching beams into each of the major linac sections.

Immediately after its installation, $t$ ' 1 e benefits of the new capability were demonstrated in a 'guide beam' tune for lead, where it is necessary to match precisely the arrival time of a light beam from the tandem with that of the heavy, stripped beam from the PII. In the past, this required careful adjustment of the pliases and amplitudes of the last resonators in PII, the first resonators in the Booster, and rebuncher parameters. This time only the single knob, 'Booster-PII relative phase', was adjusted for optimum acceleration.

c. Velocity Profile Upgrade (K. W. Shepard, B. E. Clifft, G. Wiemerslage, and G. Zinkann)

Two $\mathrm{V}$-series resonators we "e replaced with $\mathrm{H}$-series resonators during the September-October 1993 dov'ntime. Only two $\mathrm{V}$-series resonators remain in ATLAS. These two resonators will be replaced at some convenient time in the future. This most recent installation effectively completes the velocity-profile upgrade of ATLAS. 
d. Helium-Refrigeration System (J. R. Specht, B. Millar, J. A. Nolen,

During 1993 a wet expansion engine was added to one of the three ATLAS refrigerators. This upgrade provides increased cooling capacity as well as lower operating costs by replacing the Joule-Thompson valve in the refrigerator with a dual-cylinder wet expansion engine. Savings in operating costs result due to reduced use of electricity and liquid nitrogen in the refrigeration process. System efficiency improvements of $>30 \%$ were measured for this single refrigerator.The installation of three uninterruptible power supplies has begun. These units, along with modified control circuits, will allow the helium refrigerators to automatically restart in the event of a short power failure. When completed in 1995 , this system will save valuable helium as well as reduce accelerator downtime due to this type of accident.

We are presently in the design stage to add two more expansion engines to the remaining helium refrigerators. Besides the improved cooling capacity and lower operating costs, improved system reliability is expected from not needing to operate the refrigerators at $100 \%$ of their rated capacity much of the time. These engines will be installed in 1995.

Electrical energy savings at ATLAS due to this cryogenic-efficiency upgrade will be masked in 1994 by the simultaneous switch from 5-day to 7-day operations, but should show up significantly after the project completion in FY 1995.

\section{e. Radiation Interlock System}

The ATLAS Radiation Interlock System (ARIS) has functioned reliably. The ARIS system contributed approximately 37 hours to unscheduled down time. In addition, semi-annual calibrations and tests require an additional loss of approximately 2-3 weeks of potential beam time. Improvements in the system continue to be made and are implemented as part of the semi-annual test cycle. The system's most important goal of allowing experimenters access to the electronics near their target assembly was met.

\section{f. Shielding}

Access to the accelerator areas for maintenance activities while operating ATLAS was also a benefit which the new ARIS system was intended to provide, but that feature was not fully realized due to the rather high level of X-ray radiation (30-100 mrem/hr at one foot) which was often found near operating resonators. Lead sheets are now installed on all cryostats in the 'booster' and 'ATLAS' sections of the linac with a corresponding reduction in ambient radiation in the accelerator areas. The radiation levels are now low enough to usually allow access to the booster and ATLAS tunnel areas for a few minutes at a time. This significantly improves our ability to do short maintenance tasks while delivering beams.

g. The PII Charge-State Selector (S. Craig, J. Nolen, D. Phillips, D. Herbst, and G. Zinkann)

Implementation of a charge-state selector between the output of PII and the booster linac will eliminate the need to tune the booster with a "guide beam" from the tandem for the high- $\mathrm{Z}$ beam: which must be stripped after PII. Although the guide-beam method is currently being used successfully for the heavy beams, it is time consuming and injects a degree of inflexibilty into the system. When a second stripping after the booster is required, e.g. to obtain heavy beam energies above $6 \mathrm{MeV} / \mathrm{A}$, charge selection before the booster is even more important. The charge-state selector will be installed in mid 1994. (See layout of the charge-state selector shown in Fig. II-2). 


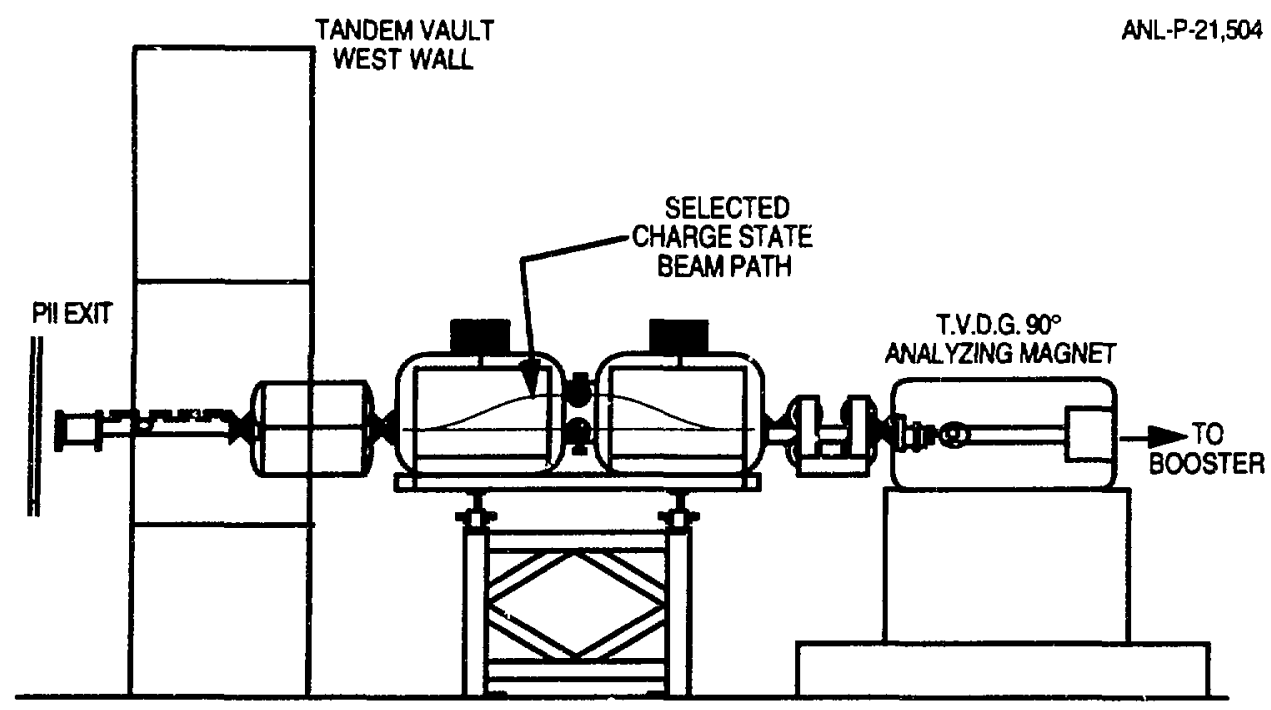

Fig. II-2. Elevation view of the new PII charge-state selector which will be located between the injector linac and the $90^{\circ}$ analyzing magnet of the tandem.

h. The Advanced ECR Project (R. Harkewicz, R. C. Pardo, P. Billquist, and Z.-Q. Xie*)

The new Positive-Ion Injector for ATLAS has experienced an extremely high level of demand since it became operational in July 1992 . Since that time approximately $50 \%$ of all beam time for the research program has been provided by the PII. This high level of demand certainly confirms all the basic arguments for the need of the PII. At the same time it emphasizes the need for a high degree of flexibility in providing the plethora of beams requested by ATLAS users.

This situation enhances provides strong arguments for a second ion source to allow both rapid switching between ion species and to provide time for the all-important tasks of source improvements and the development of new beam capabilities. In the near term, this problem is partially alleviated by the continued use of the tandem injector to provide beams in the lower third of the periodic table for the research program. This situation is only a temporary solution due both to the expected eventual retirement of the tandem and the fact that the beam mix being demanded will probably make it less possible to use the tandem for a significant fraction of the requests. Alternating between two independent ECR sources is often necessary due to memory effects and/or beam purity issues. We also note, by way of support for this position, that no other major heavyion user facility (e.g. Berkeley, Michigan State, GANIL, GSI, KVI) relies on only one ion-source system for beams.

In addition to important improvements in operational and beam development flexibility discussed above, an advanced ion source holds the potential of opening a new regime of operation for ATLAS for the heaviest of ions. For example, the new 14-GHz ECR ion source at Berkeley (AECR) has produced nearly $3 \mathrm{e} \mu \mathrm{A}$ of $238 \mathrm{U}^{33+}$ in a test run during the summer of 1993 . This test was done as a collaboration of LBL and ATLAS personnel. The spectrum obtained in this test run is shown in the lower panel of Fig. II-3. (The center panel illustrates present performance at

*Lawrence Berkeley Laboratory 
ATLAS and the upper panel shows early results). The ability to use a charge state as high as $33^{+}$ directly from the source would allow ATLAS to exceed $6 \mathrm{MeV} / \mathrm{A}$ without stripping. Such an accelerating configuration will provide benefits including eliminating the issue of foil lifetime for heavy beams and greatly simplify the issues of beam tuning. The beam emittance would be improved and slow variations in beam properties associated with foil degradation would be eliminated; such improvements are especially important for demanding experiments such as APEX. Furthermore, the absolute currents near the peak of the charge-state distributions extracted from the new generation sources such as the LBL AECR are about one-order-of-magnitude higher than from the present ATLAS source, so that currents from rare isotopes such as ${ }^{48} \mathrm{Ca}$ will be of useful intensities, even from natural feed materials in many cases. The magnet and plasma chamber for the advanced source will be constructed with AIP funds in FY 1994, while the components

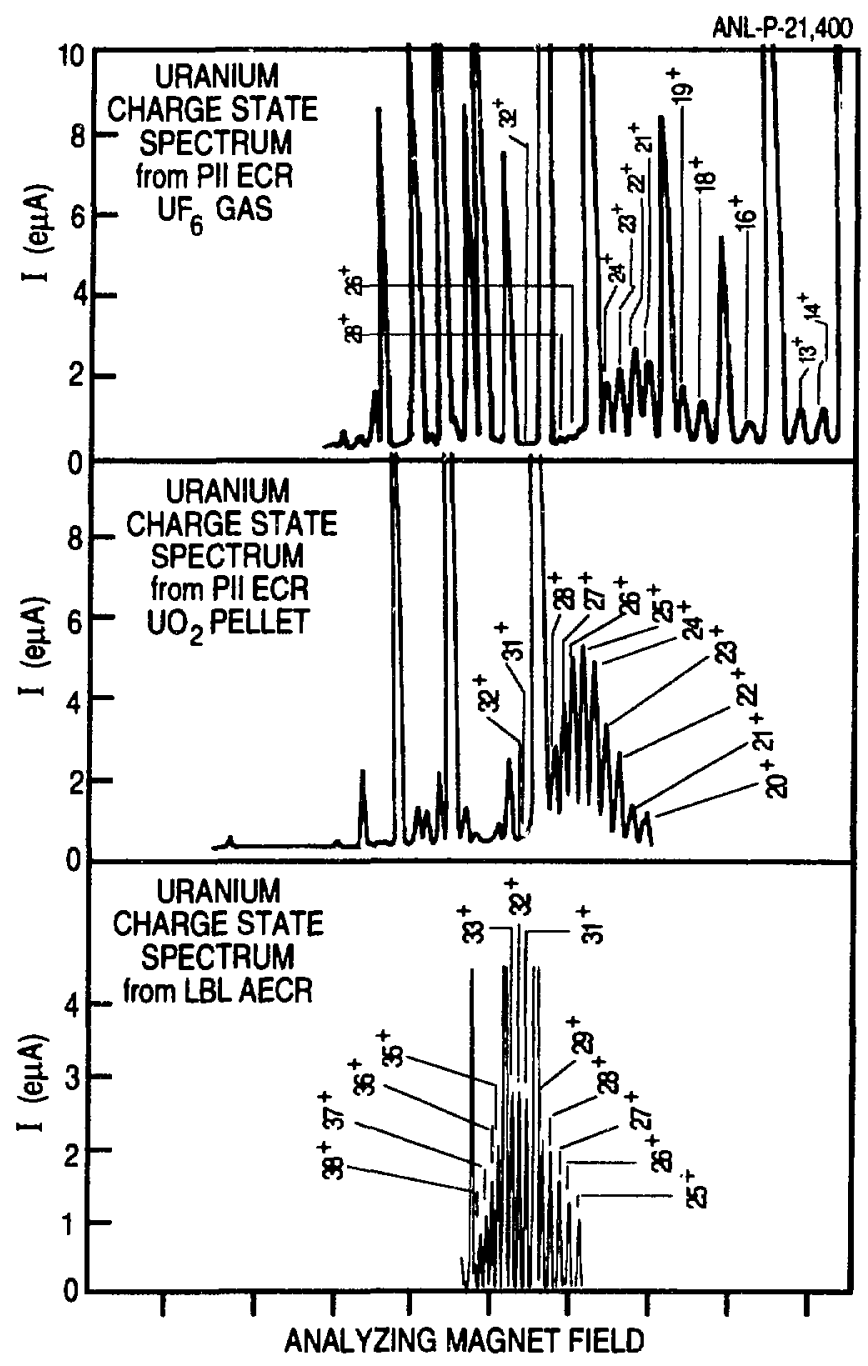

Fig. II-3. Charge-state spectra obtained in ECR ion sources with 3 different methods. Early runs with UF 6 gas in the ATLAS source (at top) peaked at about $22^{+}$with about 2 e $\mu$ amps; spectra typical of current ATLAS performance (center spectrum) using a $U_{2} O_{2}$ pellet heated by

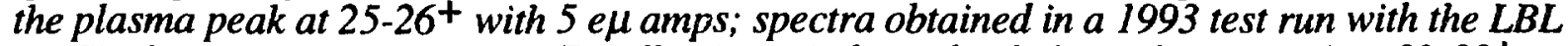
AECR (bottom panel) using a U/Re alloy in an independently heated oven peak at $32-33^{+}$at about 2.5 en amps. 


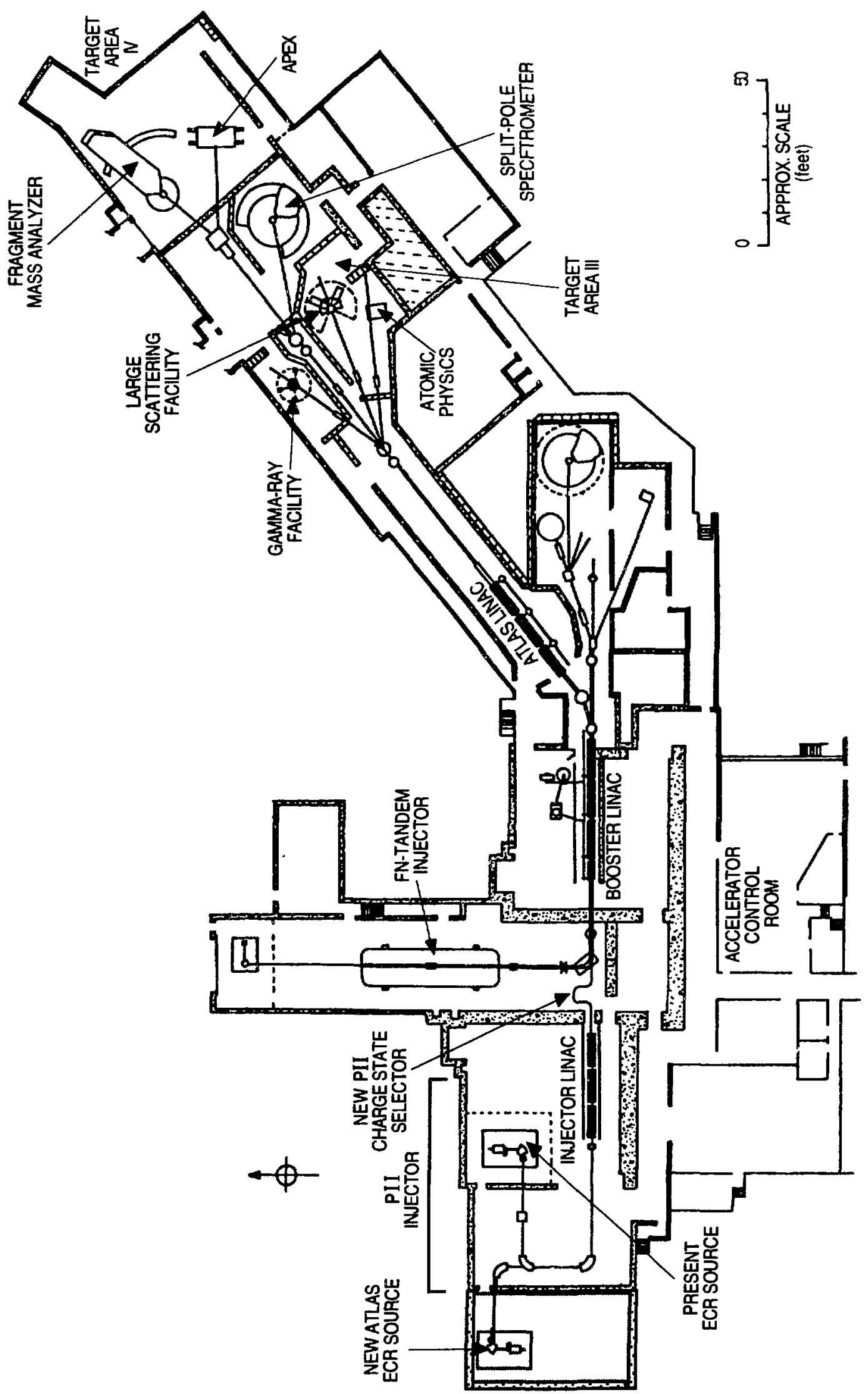

Fig. II-4. Floor plan of the ATLAS facility. The addition for the second ECR ion source (construction beginning in 1994) is the shaded area at the left side of the figure. The new charge-state selector (to be installed in 1994) is indicated schematically at the exit of the injector linac. 
necessary to operate two independent sources will be completed with AIP funds in FY 1995 and FY 1996. The second ECR project will begin in FY 1994 and be completed by FY 1996. A building addition will be constructed in 1994-95 to house the new ion source and its high-voltage platform. This addition to the ATLAS facility is shown in Fig. II-4

\title{
C. ACCELERATOR PHYSICS AND LINAC DEVELOPMENT
}

\author{
J. A: Nolen, R. C. Pardo, K. W. Shepard, R. Harkewicz, K. Joh, J. Bogaty, \\ B. Clifft, G. Zinkann, M. Kedzie, and G. Wiemerslage
}

This program involves basic research and development in the accelerator physics of superconducting linacs and related technologies. Much of this effort is related directly to upgrading or improving the technology of ATLAS with the continuing goal of enhancing its capability as a National User Facility. This work includes R\&D related to these topics: (a) superconducting accelerating structures, (b) electron-cyclotron-resonance ion sources, (c) accelerator controls, (d) beam diagnostics, and (e) time-of-flight and fast-timing technology.

Some of this activity is generic research in the basic technology of RF superconductivity with emphasis on low-frequency structures used for heavy ions at low velocities. Subjects investigated include field-limiting phenomena and multipacting. These investigations, along with continued Evolution of fabrication methods, should lead to more cost-effective structures with increased performance. Some progress was made recently towards observing and controlling the Qdegradation effect due to hydride formation in $\mathrm{Nb}$ resonator structures.

Detailed studies of the dynamics at the front end of the PII linac are also in progress, with emphasis on possibly extending the technology to even lower velocities and lower $\mathrm{q} / \mathrm{m}$ values. This work is aimed toward developing an optimal solution for the front end of a radioactive beam accelerator facility.

Some effort is also directed towards improving ECR ion-source performance at ATLAS, as well as developing techniques which would apply to increasing charge states for a future radioactive beam accelerator. A laser-ablation method of introducing solids into an ECR ion source is being developed. Successful off-line tests were completed and the apparatus is currently being installed for on-line tests. Also a low-temperature oven for feeding metals such as $\mathrm{Ca}$ and $\mathrm{Pb}$ into the source was developed and is now routinely used. A project to construct a second advanced ECR ion source with a stronger magnetic field and higher operating frequency has just begun.

\section{a. Status of the Positive-Ion Injestor}

The construction of the Positive Ion Injector (PII) was completed in the winter of 1992 and the first beam was delivered from the PII on March 29, 1992. By late 1992 uranium beams above 6 MeV/A were available for research and by mid-1993 intensities of up to $5 \mathrm{pnA}$ were delivered on target with regularity. See the section 'Operation of the ATLAS Accelerator' for a discussion of the overall operating activity of the system for the research program.

The charge-state selector to select a particular charge state after stripping at the PII exit for heavy beams such as uranium is now nearly complete. It will help operations by eliminating the detrimental effects of unwanted charge states entering the Booster Linac and being lost, in addition to eliminating the need for "guide beam" tuning. 


\section{b. ECR Source and Voltage Platform}

The ECR source and high-voltage platform have performed in a highly reliable manner during FY 1993. The performance of the source for high charge states improved dramatically when the copper extraction electrode and front face of the first stage were replaced with aluminum components. Aluminum oxide has a high secondary electron emission coefficient and therefore functions as a prolific source of cold electrons, similar to a $\mathrm{SiO}_{2}$ wall coating. The average charge state for uranium using these new aluminum components increased by nearly 2 charge units. This allowed ATLAS to use the $26^{+}$charge state for uranium during the December 1993 APEX run with no decrease in beam intensity. A typical charge-state spectrum from this configuration is shown in the center panel of Fig. II-3. Similar, though proportional, results have been observed for all other beams. The most important thing is that no frequent recoating is required as in the case of $\mathrm{SiO}_{2}$ and the overall source stability and intensity are improved.

A low-temperature oven for solid-material beams such as lead and calcium is now in routine use. It is now possible to provide calcium and similar beams on a routine basis at high intensity. The feasibility of using a 30-watt pulsed NdYAG laser to ablate or evaporate material directly into the ECR plasma has been undergoing tests for some time, in an off-line area. 1 The off-line tests indicate that our (free) surplus laser is capable of ablating significant quantities of interesting materials and we are now making arrangements to move that system over to the ECR source for on-line tests. This technique holds the possibility of using small quantities of material, with a high efficiency, and being applicable to all solids. The laser will illuminate the sample through one of the radial ECR ports, through the plasma, in the second stage. Initial source tests with the laser are planned for this spring.

The high-voltage transformers have performed flawlessly. But a new high-voltage problem has developed which does not cause much lost time but is quite baffling. After operating at platform voltages in excess of $250 \mathrm{kV}$ for some time (1-3 days), the total leakage current will begin to grow and within a few hours the power supply's maximum drain current will be exceeded. Turning off the high voltage for 10-15 minutes will cause complete recovery and the problem will not recur for some days (again 1-3 or more). The problem appears to be weakly correlated with low relative humidity in the room air and we believe it is likely due to dirt in cracks of the insulator posts which serve as the platform legs or some similar insulating component.

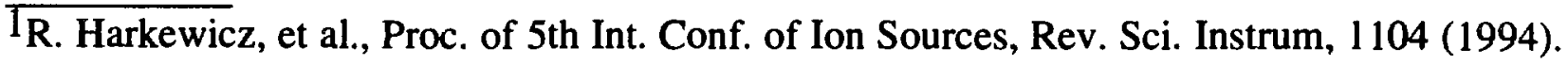

c. PII Beam Tuning. (R. C. Pardo and G. Zinkann)
}

It was possible to configure the PII section of ATLAS for most beams by scaling one of three 'tuned' configurations. Up to now such scaling was carried out manually for the PII. An automated process for this task was developed and is being debugged. The techniques employed for the PII section of the linac are also appropriate for the other linac sections and will be even more useful now that the new master oscillator system is in place which allows the phases of the major ATLAS sections to be shifted with respect to each other.

\section{d. Positive-Ion Injector and ATLAS Linac}

The Positive-Ion Injector provided beams to the experimental program for approximately $62 \%$ of all scheduled research time. The performance of the injector was highly reliable during that period. The average accelerating fields for PII resonators continued to be extremely good, averaging 3.6 $\mathrm{MV} / \mathrm{m}$ during runs using uranium beams. No deterioration in resonator performance was observed in these resonators with the exception of the need for a rare 'high-power RF conditioning' cycle which requires only a few minutes. 
The VCX development project which was described in previous FWP's has been implemented in all but one cryostat and led to quite significant improvement in the performance and reliability of the VCX system. The lost time due to failed PIN diodes was dramatically reduced and the reliability of the electronics in the VCX system is markedly improved. The system has gone from a system presenting serious reliability problems to a system that causes very little lost time. A project to improve the temperature profile of the nitrogen heat shield on the PII linac cryostats has begun. The goal of the project is to reduce the liquid nitrogen consumption and the quiescent cryostat heat load to the helium refrigeration system. In March 1994 additional heat shield components will te installed in one PII cryostat to determine the effectiveness of this approach.

\section{e. Beam Diagnostics (J. Bogaty)}

The ECR-PII injector beam line is operated at a fixed ion velocity. The platform high voltage is chosen so that all ions have a velocity of $0.0085 \mathrm{c}$ at the PII entrance. This simplifies machine configuration significantly; but, at this time, the beam arrival time at the first PII resonator must be determined using the 'fast Faraday cup' system ${ }^{2}$ discussed in past FWPs. This year we plan to install a nondestructive phase pickup detector at this location in PII based on the ATLAS beam current monitor system which was also discussed in last year's FWP. This device will providecontinuous phase information and allow quick optimization of the beam injected into PII. We also plan to install additional nondestructive beam intensity and phase monitors on some of the high-energy beam lines. Such a monitor will be particularly useful for FMA experiments where the primary beam hits one of the electric deflector plates rather than a conventional Faraday cup.

2J. M. Bogaty, et al, Proc. of the 1990 Linear Accelerator Conf., LA-12004-C, 465 (1991), Patent \#5103161.

\section{f. Technology of RF Superconductivity}

This work has several parts. One is a continuing investigation, with support from KB-02-01, of basic aspects of the technology of rf superconductivity (RFSC). Several others are collaborative projects to develop new kinds of low-beta accelerating structures. Also included are beamdynamics studies aimed at upgrading and extending the options for very low-velocity accelerating structures.

\section{g. $\quad$ Basic RFSC Technology Studies}

The resonator test facility, together with a PC-based rf control and data-acquisition system was used to observe multipacting phenomenon in ATLAS type split-ring cavities and also in a prototype cylindrically symmetric quarter-wave line structure. The quarter-wave line structure was construited as part of the joint project with the Nuclear Science Centre, Delhi, India, and will remain at Argonne for use in the fundamental studies program. The simplicity of the resonant geometry facilitates modeling of electron trajectories within the cavity, which may enhance our understanding of electron loading phenomena in superconducting resonant cavities.

\section{h. Beam Dynamics and Alternating Phase Focusing}

In early studies of the beam dynamics at the critical first few resonators of the PII linac, it was noted that these 4-gap structures possess an inherent alternating phase-focussing property enabling them to be focussing simultaneously in both longitudinal- and transverse-phase spaces. The geometrical layout of PII was not done to take advantage of this property because there were too many other unknowns about the performance of these new accelerating structures. Now that PII is completed and excellent performance of the new systems was demonstrated, we are calculating in detail the focussing and acceptance properties of these resonators. The preliminary results of these 
studies, which are part of the thesis research of a graduate student in accelerator physics, indicate that extensions of the PII linac to lower velocities (less than $0.01 \mathrm{c}$ ) could be done with extended structures (more than 4 gaps) and fewer focussing solenoids. Such concepts could be very relevant in designing a cost-effective injector for a radioactive-beam facility.

\section{i. Superconducting RFQ Development (SBIR)}

Construction of a nicbium superconducting RFQ structure is nearing completion in the Argonne Central Shops. The work is being performed as a collaboration with AccSys Technology Inc. of Pleasanton, Califernia. The work is fundec as a Phase II SBIR grant to construct and test (without beam) a superconducting RFQ device. A final copper model test has confirmed the resonant frequency, mechanical tolerances, and vibrational properties to be as designed. Niobium construction is well advanced, with most of the machining and sheet metal fabrication complete. Remaining fabrication tasks and initial tests will be completed in FY 1994.

\section{j. Superconducting Resonators for the New Delhi Booster Linac}

Construction of three prototype all-niobium quarter-wave coaxial line resonant cavities is nearly complete. The first cavity is complete and is being prepared for initial cold tests. The 97-Mhz, 15$\mathrm{cm}$ active length structures are being designed and prototyped as a joint project with the Nuclear Science Centre, New Delhi, India. Funding for the project is provided entirely from New Delhi. The structure is suitable for the velocity range 0.06 to $0.15 \mathrm{z}$. The design emphasis is to improve performance and reduce costs relative to the existing ATLAS split-ring accelerating structures. Construction and initial tests of the prototype structures are expected to be completed in 1994.

\section{Radioactive Beam Initiatives}

\section{k. Accelerator Concepts for the Front End (K. W. Shepard)}

Design of a an extended (8-gap) interdigital niobium superconducting accelerating structure is underway as a collaboration with AccSys Technology Inc. The work at AccSys is being supported through a Phase I SBIR grant. Development of an extended interdigital structure could provide several benefits:

a. Reduce overall linac cost/volt at the injector end of ATLAS by roughly a factor of two, an item of particular importance for a radioactive-beam facility.

b. Provide a high-performance alternative to RFQ structures for beam energies in the 10-200$\mathrm{keV} /$ nucleon range, enabling high accelerating gradients and large transverse acceptance for low charge-state beams. These features are highly desirable for application to a radioactivebeam facility as well as for possible industrial applications.

c. A successful prototype device could be used as either a backup or as an upgrade for the critical first three resonant cavitics in the existing ATLAS PII injector linac.

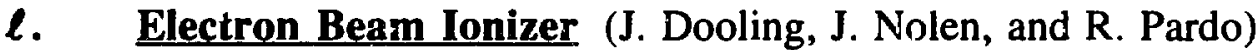

A critical question for an ISOL-type radioactive-beam facility, such as that being discussed by the North American Isospin Laboratory Committee, is the efficiency and $q / m$ of the ion source for the radioactive species. ISOLDE at CERN showed that high efficiency is obtained for a wide variety of species in the $1^{+}$charge state. One possibility is to use this technology plus an ionizer stage to increase the output of such sources to 2,3 , or $4^{+}$with high efficiency. Even going from $1^{+}$to $2^{+}$ at the input to the first accelerator structure would save millions of dollars in the facility cost. A test of this idea could be carried out using hardware currently at the tandem ion source area, with only the electron beam ionizer stage being new hardware. The equipment money for the ionizer will probably not be completely available until FY 1995, but the design and initial studies will be carried out in FY 1994. 


\section{MEDIUM-ENERGY NUCLEAR PHYSICS RESEARCH}

In order to understand how to incorporate the quark-gluon structure of the nucleon into a fundamental description of nuclear forces, the medium-energy research program in the Argonne Physics Division emphasizes the study of processes in nucisi in which interactions with the censtituents of the nucleon describe the basic physics. Specific research topics include short-range properties of nuclear forces, nuclear pion fields, and quark degrees of freedom in the nuclear medium. Because energetic leptons provide an accurate well-understood probe of these phenomena, primary emphasis is placed on experiments involving electron and deep-inelastic muon scatteling.

The Argonne medium-energy physics prugram has a major presence in the research program at CEBAF. Staff members are actively involved in construction of the experimental facilities, and are spokesmen for five experiments that have been approved for running when beam becomes available. Members of the group have assumed rcsponsibility for the construction of a broadpurpose short-orbit spectrometer (SOS) to be made generally available to the users at CEBAF. By the end of FY 1993 the SOS design was completely detailed and all of the major procurement packages executed. Commissioning of SOS is planned for late-FY 1994.

The most direct way to probe the quark structure of nuclei is to use a high-energy probe which interacis with a single quark at a time. In FNAL experiment E665, the deep inelastic scattering of 490-Ge $v$ muons at Fermi National Accelerator Laboratory measures the changes of the quark aistributions in the nucleus compared to the nucleon, and the interactions of high-energy quarks and hadrons with nuclear material. New results on the distributions of high-energy-transfer events with distinct jets of hadrons show, for the first time, the possibility of significant modifications of the gluon distributions in the nucleus. Inelastic lepton scattering makes it possible to separately control the transverse and longitudinal energy scales of exclusive reactions. In exclusive $\rho$-meson prcduction, a clear signal for color transparency is observed in the increase of the relative cross section on lead compared to that on deuterium as a function of momentum transfer squared. This complements and extends our search for color transparency in exclusive proton knock-out reactions at SLAC. New data on the ratio of deep inelastic scattering from hydrogen and deuterium will check previous results which suggest that the $\overline{\mathrm{u}}$ distribution and the $\overline{\mathrm{d}}$ distribution of the proton are quite different. A new FNAL experiment, E866, will directly measure this difference using the Drell-Yan process.

Considerable technical resources of the medium-energy progiam have been devoted to developing a new technology for producing polarized hydrogen and deuterium targets, employed in electron storage rings to study spin-dependent phenomena at high momentum transfer. Current efforts are focused on the study of elastic electron-deuteron scattering to very high momentum transfer. The tersor polarization is very sensitive to sub-nucleonic effects in nuclei, most notably mesonexcharige and quark effects. A collaboration between the Argonne group and a Russian group at Novosibirsk is engaged in a program of tensor polarization measurements. A polarized deuterium gas target intereepts the circulating beam of the VEPP-3 electron storage ring and the interactions of the deuterium gas with the circulating electrons are used to study polarization effects in elastic and inelastic scattering. Current efforts are focused on preparation of a laser-driven spin-exchange target designed at Argonne which will be used in the final phase of the experiment to reach largest momertum transfers. Installation of the final-phase target is in progress. At the same time, measurements are continuing at intermediate momentum transfers. The program at Novosibirsk provides a proof-of-principle for a proposal for HERMES, a broadly-based North AmericanEuropean collaboration, to study the spin structure of the nucleon using internal polarized targets in the HERA electron siorage ring at the DESY Laboratory, Hamburg, Germany. The HERMES proposal has been approved by the DESY directorate, and the US groups of the HERMES collaboration have assumed responsibility for fabrication and operation of specific components of the polarized target and spectrometer systems. A proposal for US funding has been approved by 
DOE. The Argonne group has the responsibility for particle identification in the HERMES experiments and for continuing development of the laser-pumped target technology.

Activities involving the NPAS program at the Stanford Linear Accelerator Center are essentially concluded. The data from measurements of the photodisintegration of the deuteron at photon energies up to $4.2 \mathrm{GeV}$ is in various stages of publication. In a companion experiment, a study of quisifree scattering, measurements have been extended to high values of momentum transfer never before accessible in (e,e'p) reactions in a search for color transparency effects. The data, in contrast to the $\rho$ production data from E665, show no clear evidence of increasing proton transparency at high momentum transfer. A series of publications summarizing the data is in preparation. A limited involvement continues in the studies at the Brookhaven National Laboratory AGS to explore global features of relativistic heavy-ion collisions under the conditions of high nuclear densities. Measurements emphasize inclusive spectra of emitted particles and iwo-particle correlations.

\section{A. SUBNUCLEONIC EFFECTS IN NUCLEI}

\section{a. Deep-Inelastic Muon Scattering from Nuclei with Hadron Detection}

(D. Geesaman, H. Jackson, S. Kaufman, E. Kinney, V. Papavassiliou, D. Potterveld, A. Zghiche, T. Kirk, * H. J. Trost, * R. Kennedy, $\dagger$ H. Kobrak, $\dagger$ P. Madden, $\uparrow$ A. Salvarani, $\uparrow$ Robert A. Swanson, $\dagger$ A. Eskreys, $\neq$ P. Malecki, $\neq$ K. Eskreys,$\ddagger$ K. Olkiewicz, $\ddagger$ B. Pawlik $\ddagger$ B. Baller, $\S$ G. B. Coutrakon, $\S$ J. Hanlon, $\S$ H. Melanson, $\S$ H. E. Montgomery,§̊ J. G. Morfin,§ C. Salgado,§ S. Wolbers,§ T..Dreyer,

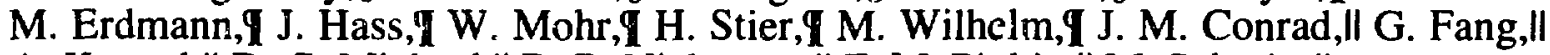
A. Kotwal,\| D. G. Michael,\| R. B. Nickerson,II F. M. Pipkin, II M. Schmitt,\| Richard Wilson,ll M. R. Adams, ${ }^{* *}$ D. A. Averill, ${ }^{* *}$ T. Carroll, ** R. Guo,** C. Halliwell,** S. Magill,** D. McLeod,** S. Aid, †† S. Kunori, $\nmid \dagger$ S. O'Day, $\dagger \dagger$ E. J. Ramberg, $\dagger \dagger$ A. Skuja, $\dagger \dagger$ P. H. Steinberg,,$\dagger$ R. L. Talaga, $\dagger \dagger$ P. Anthony, $\neq \ddagger$

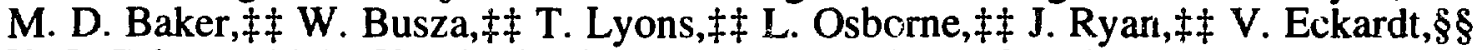

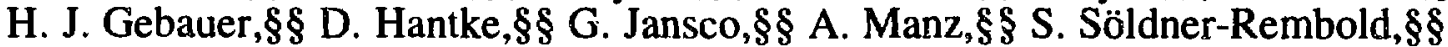

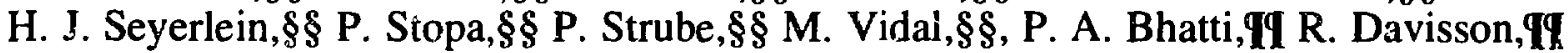

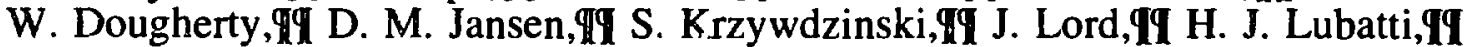

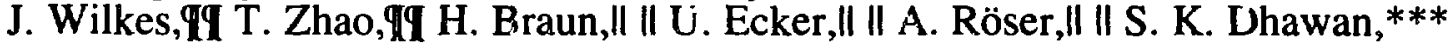
V. W. Hughes, ${ }^{* * *}$ K. P. Schüler,***, H. Venkataramania,*** F. Dietrich, $\dagger^{*} \dagger$ H. Clark, $\neq \ddagger \ddagger$ K. Hicks, $\ddagger \ddagger \ddagger$ R. Finlay, $\ddagger \ddagger \ddagger$ K. Griffioen, $\$ \S \S$ P. Spentzouris, 991 and H. Schellman (9T)

Deep-inelastic lepton scattering from nuclei provides a direct look at the quark structure of nuclear matter. These reactions revealed the first convincing evidence that the structure of nucleons is modified in the nuclear medium and have had profound implications on the understanding of nuclear dynamics. FNAL experiment E665, using the 490-GeV mion beams at Fermi National Accelerator Laboratory, provides new information on the nuclear effects on nucleon properties by studying deep-inelastic muon scattering with coincident hadron detection. The high beam energy makes the experiment particularly suited to thestudy of the region of $x<0.1$ (where $x$ is the fraction of the momentum of the nucleon carried by the struck quark in the infinite momentum frame), and total center-of-mass hadronic energy $>25 \mathrm{GeV}$, where hard QCD processes are expected to become evident and there are little data from other deep-inelastic measurements.

\footnotetext{
*High Energy Physics Division, ANL, †Univarsity of California at San Diego, $\ddagger$ Institute of Nuclear Physics, Cracow, Poland, §Fermi National Accelerator Laboratory, qUniversity of Freiburg, Germany, llHarvard University, **University of Illinois at Chicago, $\uparrow \dagger$ University of Maryland, $\ddagger$ Massachusetts Institute of Technology, $\$ \$$ Max-Planck-Institute, Germany, qTUniversity of Washington, II IIniversity of Wuppertal, Germany, ***Yale University $\dagger \dagger$ Lawrence Livermore National Laboratory, $\ddagger \ddagger$ Ohio University, $\$ \S \S U$ University of Pennsylvania, T9TNorthwestern University
} 
The data-acquisition phast of E665 was completed in January 1992 following the third block of beam time. During the 1987-1988 run, data were accumulated at two energies: $490 \mathrm{GeV}$ and 100 $\mathrm{GeV}$ on targets of liquid deuterium (luminosities of $2 \times 10^{36}$ and $2 \times 10^{35}$ muon-nucleon $/ \mathrm{cm}^{2}$ at the two energies, respectively) and gaseous $x$ enon $\left(7 \times 10^{35}\right.$ and $2 \times 10^{35}$ muon-nucleon $/ \mathrm{cm}^{2}$, respectively). Data at $490 \mathrm{GeV}$ were accumulated on a liquid-hydrogen target $\left(7 \times 10^{35}\right.$ muonnucleon $/ \mathrm{cm}^{2}$ ). In this period the target was surrounded by a streamer chamber to provide essentially $4 \pi$ acceptance. During the 1990 run, luminosities of $4 \times 10^{35}$ were collected on targets of hydrogen, deuterium, carbon, calcium, and lead. The targets were changed every 1-3 minutes to reduce systematic uncertainties in the target dependence. In the final 1991 running period, luminosities of $4 \times 10^{36}$ were collected on hydrogen and deuterium.

Final resulîs are now available from the 1987-88 data in a number of publications. Production analysis of the 1990 and 1991 data sets has been completed and physics analysis is underway. Three new results on A dependence of deep inelastic scattering demonstrate the power and kinematic range of the experiment. From the 1988 data, clear signatures of the production of events with two forward-going jets of hadrons were observed and the rates of these events were shown to quantitatively follow the predictions of perturbative QCD. ${ }^{1}$ From the 1990 data, we observed that the rate of two-forward jet events is considerably lower on nuclear targets than on deuterium. This reduction is numerically larger than that seen in the shadowing of the inc,usive muon scattering and in the events with single-forward jets of hadrons. Two-forward jet events arise from the perturbative QCD processes of gluon-bremsstrahlung and photori-gluon fusion and may provide a new measure of the gluon distribution at low $\mathrm{x}$. The reduction in the two-forward jet rate is likely to be related to a significant reduction in the gluon distribution in the nucleus at low $\mathrm{x}$. Such a reduction has been calculated to have a dramatic impact on relativis:ic heavy-ion collisions at RHIC.

In another new result, the reinteractions of the hadronic debris following deep inelastic scattering has been studied in the streamer chamber pictures. By any of three measures: the average net charge, the number of intermediate energy protons, and the backwards multiplicity, there is less cascading of the nuclear debris in the shadowing region than at higner $\mathrm{x}^{2}$

In exclusive $\rho$ vector-meson production at high energy one can independently control the transverse size $\left(Q^{2}\right)$ and the longitudinal extent $\left(E_{\rho}\right)$. This makes these reactions particularly suited for studying the color-screening effects expected at short distance scales. E665 cbserved that the relative transparency of the $\rho$-mesons through the nucleus increases with $\mathrm{Q}^{2}$ from $0.1(\mathrm{GeV} / \mathrm{c})^{2}$ to $6(\mathrm{GeV} / \mathrm{c})^{2}$ (see Fig. III-1). These results complement other searches for color transparency in the $\left(e, e^{\prime} p\right)$ and $(p, 2 p)$ reactions. In these latter reactions the momentum and energy transfer are completely coupled and no clear transparency signal has yet been observed. Several theoretical papers have suggested that exclusive vector-meson production is the simplest and clearest channel to search for color iransparency and this seems well borne out by the E665 data. Further work will focus on measuring the polarization of the $\rho$ 's from the angular distribution of the two-pion decay. Preliminary results suggest that the polarization of the $\rho$ 's changes from transverse to predominantly longitudinal at the larger momentum transfers.

The 1991 dala should provide substantive new information on the relative structure functions of hydrogen and deuterium. This will allow us to validate the NMC analysis of the Gottfried integral which suggests that there are considerable differences between the $\bar{u}$ and $\bar{d}$ distributions in the proton. These data should also provide the most accurate measurement of the possibility of nuclear shadowing in the two-body system.

1M. R. Adams et al., Phys. Rev. Lett. 69, 1026 (1992).

2R. R. R. Adams et al., submitted to Z. Phys. C. 


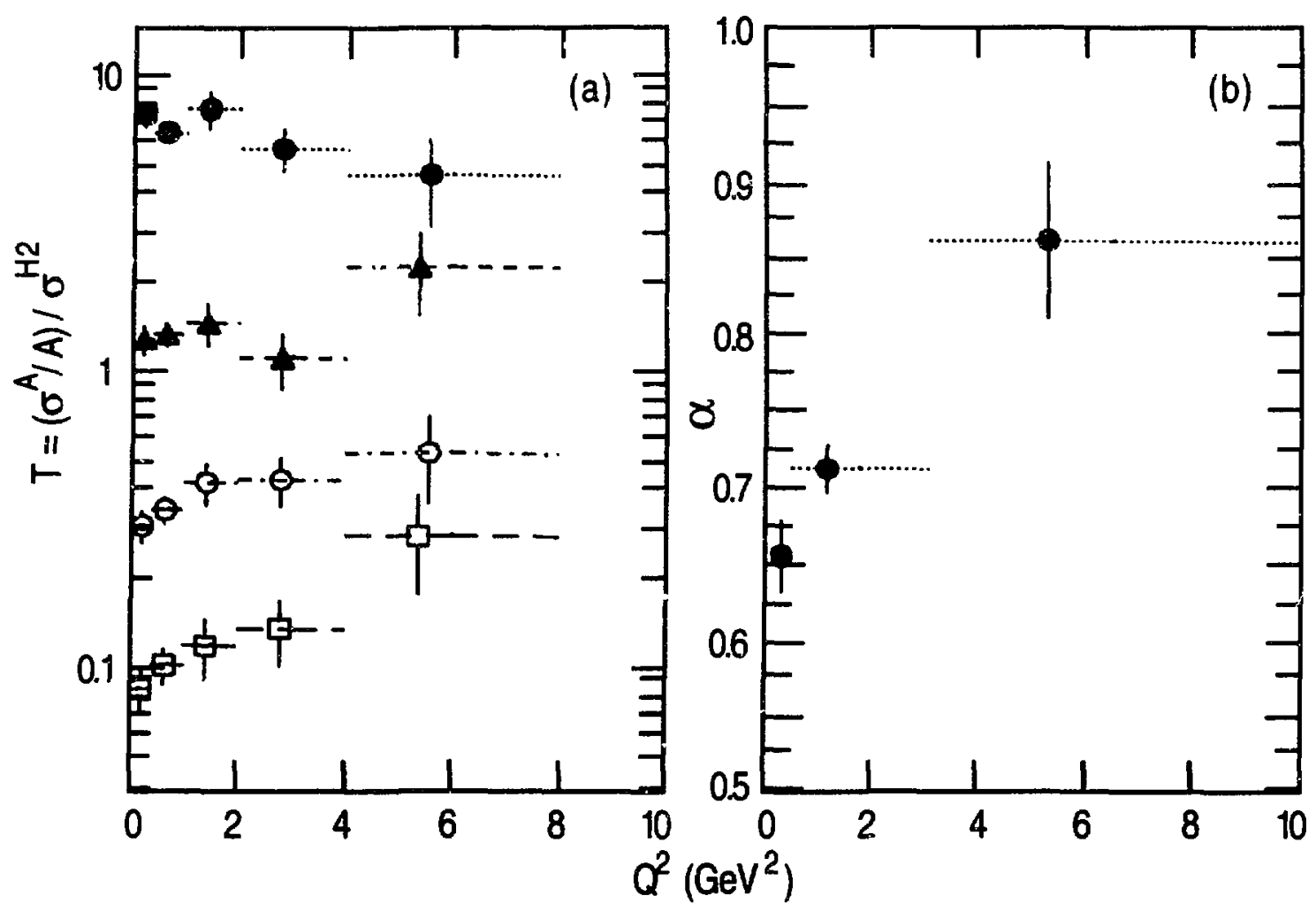

Fig. III-1. The ratio of the cross section for exclusive $\rho$ meson production on nucleas targets to that on hydrogen. a) as a function of $Q^{2}$. Note that the points have been multiplied by 8 (D2), $3(\mathrm{C}), \mathrm{l}(\mathrm{Ca})$, and $0.5(\mathrm{~Pb})$, respectively. b) $\alpha$ as a function of $Q^{2}$ where $\alpha$ is the power resulting from fits of the total production cross section for a nucleus $A, \sigma^{A}$ to $N^{*} A^{\alpha}$ at different $Q^{2} . \alpha=1$ implies a total production cross section proportional to $A$, i.e., complete transparency, while the Glauber model with normal hadrons would predict $\alpha=2 / 3$.

Argonne scientists are concentrating on expediting the production analysis, performing radiative corrections, incorporating particle identification in the analysis software and performing analysis at very low $\mathrm{x}$. The Argonne group will continue to concentrate on the 1990 data on nuclear effects in deep inelastic scattering.

b. Electron-Deuteron Scattering With a Polarized Deuterium Gas Target in the VEPP-3 Electron Storage Ring (R. J. Holt, C. E. Jones, R. Kowalczyk, D. H. Potterveld, L. Young, B. Zeidman, A. Zghiche, K. P. Coulter,* R. Gilman, $\dagger$ E. R. Kinney,‡ S. I. Mishnev,§ D. M. Nikolenko,§ S. G. Popov,§ I. A. Rachek,§

A. B. Temnyhk,§ D. K. Toporkov,§ E. P. Tsentalovich,§ D. K. Vesnovsky,§

B. B. Wojtsekhowski,§ K. de Jager, I H. de Vries, I and G. RetzlaffI)

Measurements of $\mathrm{T}_{20}$ in electron-deuteron scattering permit a separation of the charge and quadrupole form factors of the deuteron. With the existing $\mathrm{T}_{20}$ data, the charge form factor of the deuteron and the isoscalar charge form factor extracted from data for electron scattering from ${ }^{3} \mathrm{He}$

*University of Michigan, $\nmid$ Rutgers University, $\ddagger$ University of Colorado, §Budker Institute for Nuclear Physics, Novosibirsk, Russia, TNIKHEF, Amsterdam, The Netherlands 
and ${ }^{3} \mathrm{H}$ cannot be simultaneously explained by existing theoretical calculations. This implies that either the existing $\mathrm{T}_{20}$ data are in error or that some physics is missing from the calculations. Thus, it is essential to provide an independent measure of $T_{20}$ with a different method, the polarized internal target method. A high-density polarized deuterium gas target (Phase 2 storage cell), designed and constructed at Argonne, was installed and tested in the VEPP-3 ring. Results indicate that the target has a polarization $P_{z z}=0.6 \pm 0.2$ and a thickness of $4 \times 10^{12}$ nuclei $/ \mathrm{cm}^{2}$ With this target, we plan to provide $T_{20}$ analyzing power measurements up to $Q^{2}=15 \mathrm{fm}^{-2}$.

The first results available from the phase 2 measurement in Novosibirsk are not in good agreement with the MIT-Bates measurements. The phase 2 experiment is being repeated, while phase 3 is under preparation. Phase 3 involves the use of a laser-driven target. An engineering test run with the phase 3 target is being planned for Fall 1994.

c. Laser-Driven Polarized Deuterium Gas Target (R. J. Holt, C. E. Jones, R. S. Kowalczyk, D. H. Potterveld, L. Young, B. Zeidman, K. P. Coulter,* E. R. Kinney, $\dagger$ J. van den Brand, J. Neal, $\ddagger$ and D. K. Toporkov§)

Systematic studies of the polarization vs. flow achievable with the laser-driven source have been completed. Figure III-2 shows the atomic polarization of hydrogen, deuterium, and potassitim as a function of $\mathbf{H} / \mathrm{D}$ flow.

The first measurement of nuclear polarization was made for a laser-driven polarized deuterium target. The target cell was filled at a rate of $10^{18} \mathrm{~d} / \mathrm{s}$ from the laser-driven source. The polarization of atoms input into the target cell exceeded $50 \%$ and the total target thickness was greater than $2 \times 10^{14} \mathrm{~cm}^{-2}$.

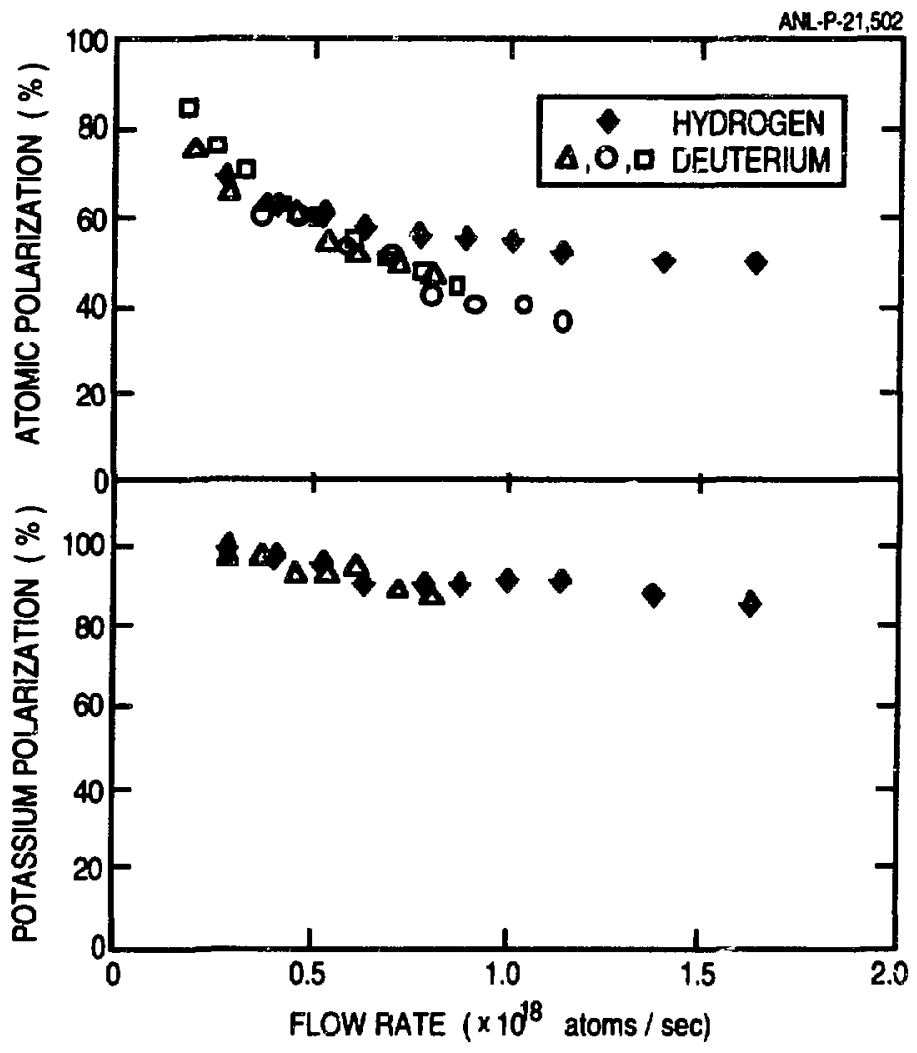

Fig. III-2. Atomic polarization: vs. flow for hydrogen and deuterium. Also shown is the potassium polarization for a given $H / D$ flow. 
Deuterium atoms in the target cell were ionized and accelerated to $40 \mathrm{keV}$ before impinging on a tritium target. The asymmetry for neutrons emitted from the well-known $T(d, n)^{4}$ He reaction was measured. The observed non-zero value of $\mathrm{P}_{\mathrm{zz}}, 0.053 \pm 0.007$, provides the first direct evidence for the Walker-Anderson effert. Walker and Anderson speculated that polarized gas at high densities and even at relatively high magnetic fields approaches spin temperature equilibrium because of the relatively high deuterium-deuterium spin-exchange scattering rate. This implies that gas target nuclei at high density will become polarized without the necessity for inducing RF transitions. Based on a spin temperature model, the deduced nuclear vector polarization of the target is $27 \%$.

In order to improve the polarization of the target, the ion extractor lenses which currently act as a depolarizing surface and a conductance limiter for the target cell will be redesigned to have negligible influence on the target polarization. This improvement is expected to increase the target polarization at least two-fold.

*University of Michigan, †University of Colorado, $\ddagger$ University of Wisconsin, §Budker Institute for Nuclear Physics, Novosibirsk, Russia

d. Two-Body Photoc integratiori of the Deuteron at High Energy: Experiment NE17 at SLAC (R. J. Holt, D. F. Geesaman, H. E. Jackson, C. E. Jones, V. Papavassiliou, D. H. Potterveld, B. Zeidman, K. P. Coulter, E. R. Kinney, $\dagger$ R. Arnold, $\ddagger$ P. Bosted, $\ddagger$ C. Keppel,$\ddagger$ A. Lung, $\ddagger$ S. Rock,$\ddagger$ M. Spengos, $\ddagger$ L. H. Tao, $\ddagger$ J. White \pm Z. Szalata,$\ddagger$ M. Epstein, $\S$ D. Margaziot,$\S$ J. Arrington, $\S$ E. Beise,, E. Belz, 1 B. Filippone,\$ H. Gao, $\mathbf{W}$ W. Lorenzon, $\mathbf{X}$ R. McKeown, $\mathbf{A}$ B. Mueller, $\mathbf{T}$ T. O'Neill, J. Napolitano,\|l P. Anthony, ${ }^{* *}$ K. V. Bibber, ${ }^{* * F}$. Dietrich, ${ }^{* *}$ M. Chapman, $\dagger \dagger$ R. Ent, $\neq \ddagger$

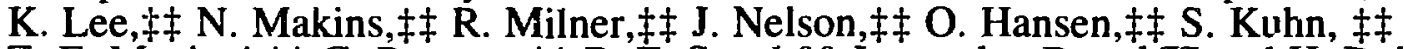
Z.-E. Meziani, $\ddagger$ G. Petratos, $\neq \ddagger$ R. E. Segel,§§ J. van den Brand,TI and H. BultenTI)

Experiment NE17 was granted three days of beam time during the last NPAS run at SLAC. During that time, the data for the $\gamma \mathrm{d} \rightarrow$ pn reaction were extended up to $2.8 \mathrm{GeV}$ at $\theta_{\mathrm{cm}}=90^{\circ}$

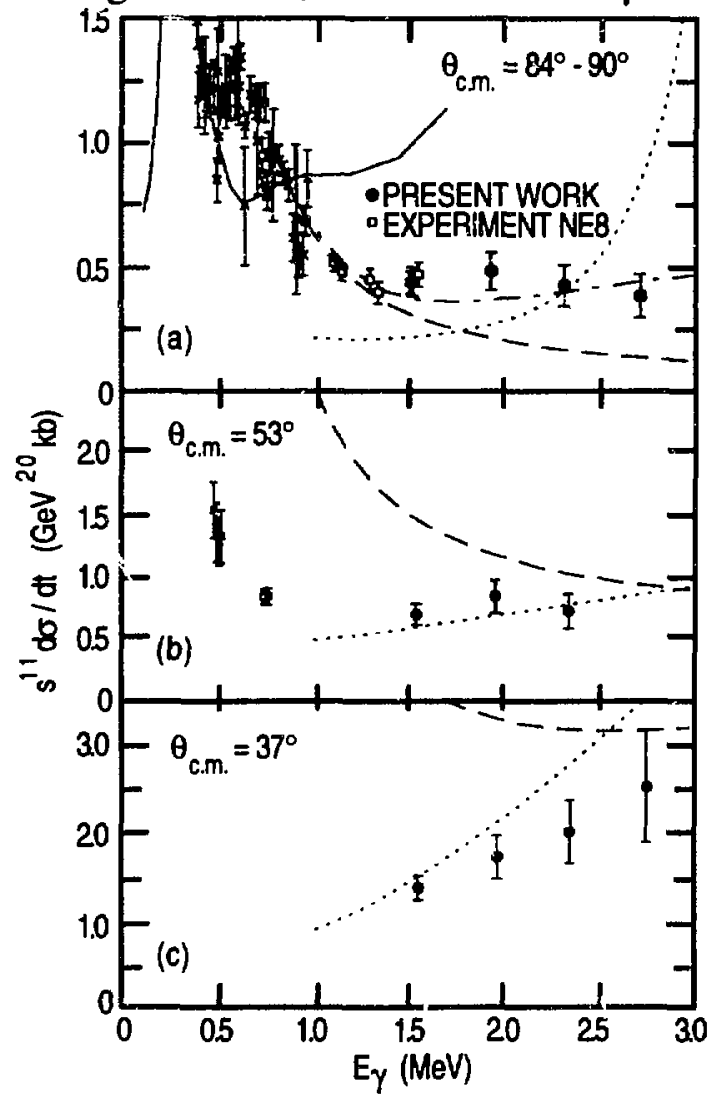

Fig. III-3. $s^{l l} d \sigma / d t$ vs. $E_{\gamma}$ for $\theta_{c m}=90^{\circ}(a)$ $\theta_{\mathrm{cm}}=53^{\circ}(\mathrm{b})$ and $\theta_{\mathrm{cm}}=37^{\circ}$ (c). The present work is plotted as circles, and earlier data are plotted as squares and crosses. The dashed curve represents the reduced nuclear amplirudes analysis, the dotted line is the QGS model and the solid line is a traditional meson-exchange calculation, as discussed in the text. 
and up to $4.2 \mathrm{GeV}$ at $\theta_{\mathrm{cm}}=37^{\circ}$. Data were also taken at $\theta_{\mathrm{cm}}=37^{\circ}, 53^{\circ}$, and $90^{\circ}$ at $\mathrm{E}_{\gamma}=1.6$ $\mathrm{GeV}$ so that a complete angular distribution would be available at this energy where an enhancement at a large angle $\left(143^{\circ}\right)$ was found during experiment NE8. The data analysis was completed. The results indicate the surprising feature that the data at $90^{\circ}$ and $54^{\circ}$ follow the quark counting rule prediction, while those at $37^{\circ}$ do not (see Fig. III-3).Perhaps this indicates that $\mathrm{p}_{\mathrm{T}}^{2}$ is the controlling variable in the approach to asymptotic scaling.

\begin{abstract}
*University of Michigan, †University of Colorado, $¥$ American University, §California State Polytechnic University, TCalifornia Institute of Technology, IIRensselaer Polytechnic Institute, **Lawrence Livermore National Laboratory, $†+$ Massachusetts Institute of Technology, $\ddagger \ddagger$ Stanford University, $\$ \$ N o r t h w e s t e r n$ University, TTUniversity of Wisconsin.
\end{abstract}

\title{
e. Nuclear and $Q^{2}$ Dependence of Quasielastic (e,e'p) Scattering at Large Momentum Transfer (H. E. Jackson, K. Coulter, D. F. Geesaman, R. J. Holt, E. R. Kinney, D. Potterveld, B. Zeidman, E. Beise,* B. Filippone,* W. Lorenzon,* T. O'Neill,* R. Arnold, $†$ S. Rock, $\dagger$ Z. Szalata, $†$ M. Epstein, $\ddagger$ J. J. Napolitano,§ R. C. Minehart, , R. Ent,\| R. Milner,\| D. Beck,** and J. van den Brand $\dagger \dagger$ )
}

An experiment has been completed at the Stanford Linear Accelerator Center in which measurements of the (e,e'p) coincidence quasielastic cross section in nuclei have been extended to the largest possible $\mathrm{Q}^{2}$ attainable with the Nuclear Physics Injector and the End Station $\mathrm{A}$ spectrometers. Coincidence measurements of the quasielastic $\left(e, e^{\prime} p\right)$ cross section were made on nuclei from carbon to gold in the $Q^{2}$ range of $1-7(\mathrm{GeV} / \mathrm{c})^{2}$.

The 1.6-GeV/c spectrometer was used for detection of quasielastically-scattered electrons and the 8-GeV/c spectrometer for recoil proton detection. The $8-\mathrm{GeV} / \mathrm{c}$ spectrometer, operated in the large acceptance mode, provided $4 \mathrm{msr}$ solid angle for proton momenta up to $5.5 \mathrm{GeV} / \mathrm{c}$. Because of the significant kinematic focusing of the recoil protons which occurs at high $\mathrm{Q}^{2}$, this allows $100 \%$ acceptance of the Fermi cone for $\mathrm{Q}^{2} \geq 6(\mathrm{GeV} / \mathrm{c})^{2}$. The missing energy resolution was $6 \mathrm{MeV}$ at the lowest $\mathrm{Q}^{2}$ and increased to $25 \mathrm{MeV}$ at $\mathrm{Q}^{2}=7(\mathrm{GeV} / \mathrm{c})^{2}$. Data were accumulated for hydrogen, deuterium, carbon, iron, and gold targets with an average luminosity of $3 \times 10^{37} \mathrm{e}$-nucleons/cm ${ }^{2}$ $\mathrm{sec}$. The beam energy ranged from 2 to $5.8 \mathrm{GeV}$ in measurements of momentum transfers of 1,3 , 5 , and $6.8(\mathrm{GeV} / \mathrm{c})^{2}$. Analysis of the carbon data produced clean missing-energy spectra in which the contributions of the $s$ and $p$ shells could be clearly discerned. The results agree well with spectral functions obtained for the same nuclei at much lower momentum transfer, confirming the validity of the data reduction procedures and assumptions. At sufficiently high $\mathrm{Q}^{2}$ there is a striking prediction of QCD for the (e,e'p) quasielastic process in nuclei. At large momentum transfer, theoretical considerations suggest diminishing elastic and inelastic final-state interactions of the recoil proton in the nuclear medium as $\mathrm{Q}^{2}$ increases. This effect is called "co'or transparency". In order to search for this process, the cross section for quasielastic (e,e'p) scattering has been measured over a range of missing energyup to the pion threshold and missing momentum up to the Fermi momentum. The missing energy and momentum distributions and differential scattering cross sections are consistent with scattering from a single nucleon as the dominant process. In the analysis, the nuclear transparency is defined as the ratio of the measured cross section to the plane-wave impulse approximation. No significant rise of the transparency with $\mathrm{Q}^{2}$ is ooserved. Preliminary results are shown in Fig. III-4. For all targets, over the full range of $1-7(\mathrm{GeV} / \mathrm{c})^{2}$ in momentum transfer, the results are consistent with the conventional Glauber approximation. No trend of diminished final-state interactions of the recoil proton is evident.

\footnotetext{
*California Institute of Technology, †American University, $¥$ California State University, §Rensselaer Polytechnic Institute, TUniversity of Virginia, IIMassachusetts Institute of Technology $* *$ University of Illinois, $†$ †University of Wisconsin
} 


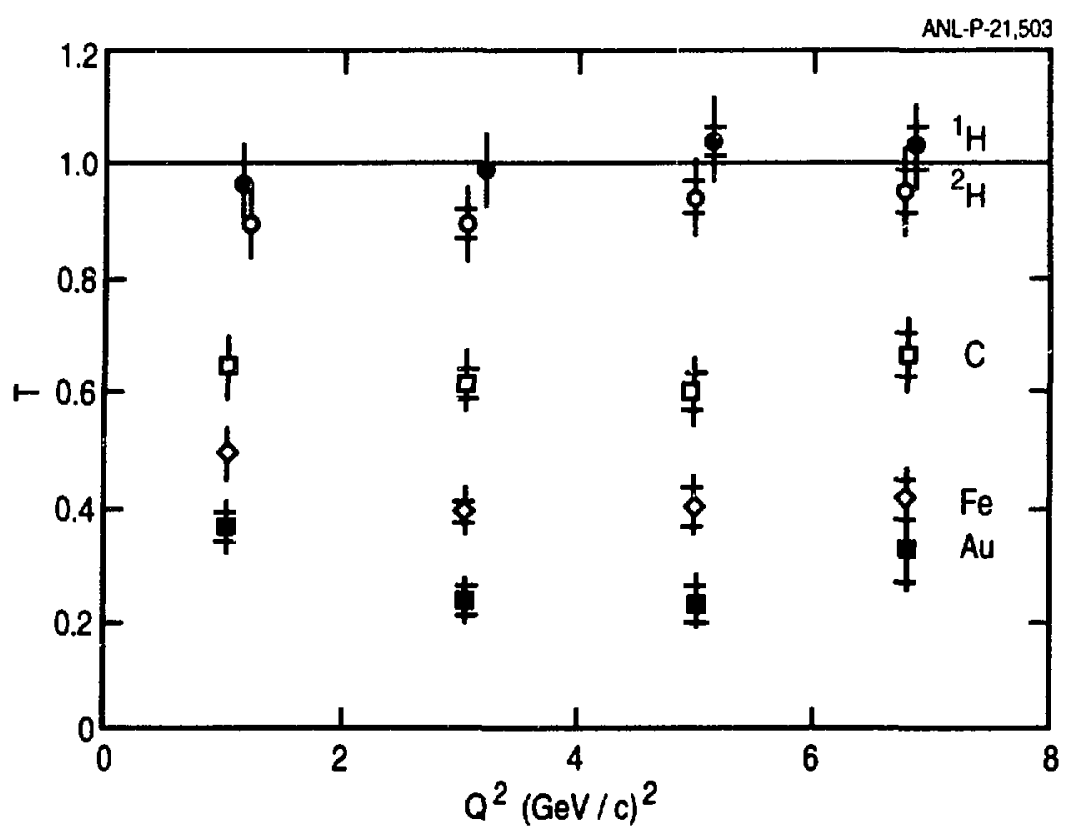

Fig. III-4. Preliminary nucleus transparency results from quasielastic $\left(e, e^{\prime} p\right)$ scattering on ${ }^{1} H$ (solid cirlces), ${ }^{2} \mathrm{H}$ (open circles), $12 \mathrm{C}$ (open squares), $56 \mathrm{Fe}$ (open diamonds), and $197_{\mathrm{Au}}$ (solid squares). The inner error bars represent statistical errors. The outer error bars include statistical plus systematic errors added in quadrature.

f. Measurement of the Helicity-Dependent Asymmetry in ${ }^{3} \overrightarrow{\mathbf{H}}\left(\overrightarrow{\mathbf{e}}, \mathrm{e}^{\prime}\right)$

Quasielastic Scattering (C. E. Jones, J. Arrington,* E. J. Beise,* R. W. Carr,* B. W. Filippone,* H. Gao,* A. Lung,* R. D. McKeown,* B. Mueller,* M. Pitt,* G. Dodson, $\uparrow$ K. Dow, $\uparrow$ D. Deschepper, $\uparrow$ R. Ent, $\uparrow$ M. Farkhondeh, $\uparrow$ J.-O. Hensen, $\uparrow$ W. Korsch, $\uparrow$ L. Kramer, $\dagger$ K. Lee, $\dagger$ N. Makins, $\uparrow$ R. Milner, $\dagger$ D. Tieger, $\uparrow$ P. Welch, $\dagger$ E. Candell, $\ddagger$ J. Napolitano, $\ddagger$ and W. Lorenzon§)

A measurement of the helicity-dependent asymmetry in quasielastic scattering of polarized electrons from polarized ${ }^{3} \mathrm{He}$ at the MIT-Bates electron accelerator was completed in June 1993. Asymmetries sensitive to both the electric and magnetic form factors of the neutron were measured as a function of the energy transfer over a broad range of energy transfer at $\mathrm{Q}^{2}=0.14(\mathrm{GeV} / \mathrm{c})^{2}$ and $\mathrm{Q}^{2}=0.20(\mathrm{GeV} / \mathrm{c})^{2}$. In addition, the elastic asymmetry and the quasielastic asymmetry at low energy transfer were measured. The combination of asymmetry information over such a broad kinematic range will provide significant constraints on theoretical mnodels of the three-body system. The data from this measurement give substantial improvement of both the statistical and systematic precision over previous asymmetry measurements. Preliminary analysis of the data has been completed and the final results will soon be available for publication.

*California Institute of Technology, † Massachusetts Institute of Technology, and Bates Linear Accelerator Center, $\ddagger$ Rensselaer Polytechnic Institute, §TRIUMF, Vancouver, Canada 
g. Investigation of the ${ }^{3} \mathrm{He}$ Wave Function by Quasifree Scattering (C. E. Jones, C. Bloch,* C. D. Goodman,* W. W. Jacobs, * M. Leuschner,* H. O. Meyer,* T. Rinckel,* A. Smith, ${ }^{*}$ J. Sowinski, ${ }^{*}$ F. Sperisen, ${ }^{*}$ B. von Przewoski, ${ }^{*}$ H.-J. Bulten, $\dagger$ M. Miller, $\dagger$ J. Neal, $\dagger$ O. Unal, $\dagger$ J. van den Brand, $\dagger$ Z.-L. Zhou, $\dagger$ R. Ent, $\ddagger$ J.-O. Hansen, $\ddagger$ K. Lee, $\ddagger$ S. Pate $\ddagger$ W. Korsch, $\ddagger$ R. G. Milner, $\ddagger$ C. Tschalaer, $\ddagger$ W. Lorenzon,, D. Marchlenski, $\mathbb{I}$ E. R. Sugerbaker, I P. Pancella,II and W. K. Pitts**)

The CE25 experiment at IUCF finished data taking in March 1993. In this experiment, the target and beam analyzing powers and the spin correlation parameter in ${ }^{3} \overrightarrow{\mathrm{He}}(\overrightarrow{\mathrm{p}}, 2 \mathrm{p})$ and ${ }^{3} \overrightarrow{\mathrm{He}}(\overrightarrow{\mathrm{p}}, \mathrm{pn})$ quasielastic scattering were measured at incident proton energies of 200,290 , and $400 \mathrm{MeV}$. From this data one can extract information about the spin-dependent momentum distribution of the nucleons in ${ }^{3} \mathrm{He}$ and about the small $D$ and $S^{\prime}$ components of the ${ }^{3} \mathrm{He}$ wave function. Information about final-state interactions in the process can be obtained by looking at the variation of the results with the incident beam energy. This information is very important for electron scattering experiments that hope to extract information about the electromagnetic properties of the neutron using polarized ${ }^{3} \mathrm{He}$ targets. In addition to collecting information on the spin observables, the experiment demonstrated the iniernal target technique with the polarized ${ }^{3} \mathrm{He}$ internal target that is to be used for the measurement of the neutron spin structure function by the HERMES collaboration at DESY. The CE25 data analysis is currently underway and results are expected this year.

*Indiana University, Cyclotron Institute, †University of Wisconsin, $\ddagger$ Massachusetts Institute of
Technology, and Bates Linear Accelerator Center, §TRIUMF, Vancouver, Canada, OOhio State
University, ॥Western Michigan University, **University of Louisville

h. Studies of Particle Production in Collisions of Relativistic Heavy Ions with Nuclei (S. B. Kaufman, D. Beavis,* C. Chasman,* Z. Chen, * Y. Y. Chu,* J. B. Cumming,* R. Debbe,* M. Gonin,* S. Gushue,* O. Hansen,* S. Hayashi,* M. J. LeVine,* B. Moskowitz,* J. Olness,* L. P. Remsberg,* M. J. Tannenbaum,* J. H. van Dijk,* F. Videbaek,* B. Cole, $†$ K. Kurita, $\uparrow$ S. Nagamiya, $\uparrow$ P. Stankus, $\dagger$

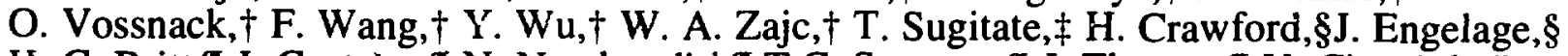
H. C. Britt, I J. Costales, I N. Namboodiri, I T.C. Sangster, I J. Thomas, I V. Cianciolo,II W. Kehoe,\| D. Morrison,\| S. Park,\| P. Rothschild,\| D. S. Woodruff,\| H. Hamagaki, ** R. Hayano,** S. Homma,** Y. Miake, ${ }^{* *}$ H. Sakurai,** S. Y. Fung, $\dagger \dagger$ J. Kang, $\dagger \dagger$ and R. Seto $\dagger \dagger$ )

The interactions of relativistic heavy ions with nuclear targets is being studied at the BNL AGS by measuring cross sections for produced particles $(\pi, K, p)$ and two-particle correlations $(\pi \pi, K K, p p)$. Event characterization (i.e, central, peripheral, minimum bias) is done using calorimetry, charged-particle multiplicity, and transverse energy. The present experiment, E866, is the third in this series, following E802 and E859. The main objective in E866 is to measure particle production from interactions of $10.5-\mathrm{GeV} / \mathrm{A} \mathrm{Au}$ beams with a $\mathrm{Au}$ target. During September-October 1993 a successful run was completed, during which data were taken with an upgraded spectrometer system, including a new forward-angle spectrometer. The Level-2 trigger to enhance the recording of two-particle correlations and iow-cross-section particles was also used. The Argonne contribution to the current experiment is limited, due to constraints of other commitments; the primary activity is to participate in the testing and calibration of phoswich scintillator arrays and a zero-degree scintillator hodoscope in collaboration with the LLNL group.

*Brookhaven National Laboratory, †Columbia University, $¥$ Hiroshima University, Japan, §University of California, ILawrence Livermore National Laboratory, IIMassachusetts Institute of Technology, **University of Tokyo and INS, Japan, ††University of California 
i. Electroproduction of Kaons and Light Hypernuclei (D. F. Geesaman,

R. J. Holt, H. E. Jackson, C. E. Jones, D. H. Potterveld, S. B. Kaufman, J. P. Schiffer,

V. Papavassiliou, B. Zeidman, R. E. Chrien,* S. Bart,* R. Sawafta,* R. J. Sutter,*

B. W. Filippone, $\dagger$ W. Lorenzon, $\dagger$ J. Napolitano, $\ddagger$ R. Carlini,§ R. Ent, $\S$ D. Mack, $\S$

S. A. Wood,§ E. R. Kinney, T O. K. Baker,\| W. W. Buck,\| L. Tang,॥

E. V. Hungerford, ${ }^{* *}$ K. Lán, ${ }^{* *}$ B. W. Mayes, ${ }^{* *}$ J. J. Reidy, $\dagger+$ N. Makins, $\ddagger \ddagger$

R. E. Milner, $\neq \ddagger$ R. E. Segel, $\S \S$ A. Klein, $\$ \S R$. Gilman, ॥ ॥ and J. F. J. van den Brand***)

Inasmuch as both the electron and $\mathrm{K}^{+}$are particles that interact relatively weakly, electroproduction of light hypernuclei provides a relatively low-distortion means of investigating the fundamental interactions between nucleons, lambdas, and sigmas in few-body systems. In order to perform a detailed investigation of the basic hyperon-nucleon interactions, a proposal to study the electroproduction of kaons in deuterium and other light nuclei was submitted to CEBAF and approved by PAC 5. The particular reactions to be studied are $\left(e, e^{\prime} K^{+}\right)$reactions on targets of $D$, ${ }^{3} \mathrm{He}$, and ${ }^{4} \mathrm{He}$ at incident electron energies near $3 \mathrm{GeV}$ with coincident detection of the emergent $\mathrm{e}$ and $\mathrm{K}^{+}$in the HMS and SOS magnetic spectrometers in Hall C. The residual nuclei will be left in bound or nearly-bound states. In addition to providing new information on the phases and momentum dependence of hyperon-nucleon interactions and measurements of hypernuclear formation, the study will investigate bumps in the cross sections that are anticipated near values of the missing mass that correspond to threshold production of sigmas.

*Brookhaven National Laboratory, †California Institute of Technology, $¥$ Rensselaer Polytechnic Institute, §CEBAF, IUniversity of Colorado, IHampton University, **University of Houston, $\dagger+$ University of Mississippi, + Massachusetts Institute of Technology, $\$ \S N o r$ hwestern University TIOld Dominion University, II IIRutgers University, *** University of Wisconsin

j. Two-Body Photodisintegration of the Deuteron at Forward Angles and Photon Energies Between 1.5 and 4.0 GeV (R. J. Holt, S. J. Freedman, D. F. Geesaman, H. E. Jackson, S. Kaufman, D. Krakauer, D. H. Potterveld, B. Zeidman, K.P. Coulter, ${ }^{*}$ E. R. Kinney, $\dagger$ J. Napolitano, $\ddagger$ R. Carlini,§ R. Gilman, R. E. Segel,\| P. Bosted,** E. Beise, $\nmid+$ B. Filippone, $\nmid \dagger$ R. D. McKeown, $+\dagger$ R. Milner, D. Beck, \&§ Z.-E. Meziani,TI R. Minehart,II II and O. Keith Baker***)

It was discovered in experiments NE: ${ }^{1}$ and NE 17 at SLAC that the differential cross section near $\theta_{\mathrm{cm}}=90^{\circ}$ for the $\gamma \mathrm{d} \rightarrow$ pn reaction at the highest measured photon energies $\left(\mathrm{E}_{\gamma}=1.3-2.8 \mathrm{GeV}\right)$ has an energy dependence consistent with the constituent counting rules. Experiment NE17 at SLAC indicates the s-dependence of the cross section is not consistent with constituent counting at forward angles (see Fig. III-3).

At CEBAF we have proposed to measure the differential cross section at forward angles for two of the simplest exclusive binary reactions involving a deuteron in the initial or final state: (1) $\gamma d \rightarrow$ pn between $\mathrm{E}_{\gamma}=1.5$ and $4.0 \mathrm{GeV}$, and (2) $\gamma \mathrm{d} \rightarrow \pi^{0} \mathrm{~d}$ between $\mathrm{E} \gamma=1.0$ and $3.0 \mathrm{GeV}$. This proposal was accepted by CEBAF PAC4 and recently accorded high scientific priority by PAC7.

The constituent counting rules predict an energy dependence of $\mathrm{s}^{-11}$ and $\mathrm{s}^{-13}$ for the $\gamma \mathrm{d} \rightarrow \mathrm{pn}$ and $\gamma \mathrm{i} \rightarrow \pi^{0} \mathrm{~d}$ cross sections, respectively, where $\mathrm{s}$ is the square of the energy in the center of mass. Since more complex nuclei involve more constituents and consequently a more rapid fal'-off of the cross section as a function of $s$, these two cases may represent the only practical nuclear reactions that can be studied at large values of $s$ where asymptotic scaling is most likely to be observed.

${ }^{1}$ S. J. Freedman et al., Phys. Rev. C 48, 1864 (1993)

*University of Michigan, †University of Colorado, $\ddagger$ Rensselaer Polytechnic Institute, §CEBAF and College of William and Mary, TRutgers University, IINorthwestern University, **American University and SLAC, $\dagger \dagger$ California Institute of Technology, $\ddagger \ddagger$ Massachusetts Institute of Technology, §§University of Illinois, TIStanford University, II IUniversity of Virginia, ***Hampton University 
These experiments are expected to be practical at high energies since a large beam current $(\sim 30$ $\mu \mathrm{A}$ ) and a large solid-angle spectrometer (HMS in Hall C) are expected to be available at CEBAF. In particular, the CEBAF experiments will permit exploration of not only the scaling with photon energy, but also the transverse momenta in the reaction process.

k. A Study of Longitudinal Charged-Pion Electroproduction in D, ${ }^{3} \mathrm{He}$, and ${ }^{4} \mathrm{He}$ (H. E. Jackson, K. P. Coulter, D. F. Geesaman, R. J. Holt, S. Kaufman, D. Potterveld, B. Zeidman, R. Gilman,* E. R. Kinney, † J. Mougey, † B. Saghai, and R. E. SegelT)

Recent studies of pion electroproduction on the deuteron carried out by the ANL group at ALS, Saclay, show that even in the weakly-bound deuteron, multinucleon processes alter the electroproduction amplitudes in the forward direction. The data provide the first experimental indications for a significant change in the pion-nucleon coupling for nucleons bound in nuclei. It is clear that forward-angle electroproduction may be a sensitive probe of the properties of the pion coupling in the nuclear medium. At CEBAF, we will study longitudinal charged-pion electroproduction (in the excitation region below the delta isobar) along the direction of the momentum transfer where the charge scattering process dominates. Direct comparison of the cross section per nucleon in deuterium and the helium isotopes with the experimental value for the free nucleon will provide estimates of the strength of the nuclear pion fieid. A Rosenbluth separation of the longitudinal and transverse cross sections will be performed for four-momentum transfers of 2.5 and $10 \mathrm{fm}^{-2}$.

Measurements for a number of light nuclei will provide useful data on the sensitivity of longitudinal electroproduction to nuclear binding effects. If current conceptions of pion-exchange currents in nuclei are correct, longitudinal electroproduction will be suppressed at the lower momentum transfer and enhanced at the higher momentum transfer by multinucleon processes. If on the other hand, as suggested by recent data from Drell-Yan studies of antiquark structure functions, there is no such enhancement, a reformulation of pion exchange models of the mediumand short-range properties of nuclear forces will be required. Our proposal to carry out such a series of measurements at CEBAF using the coincident-pair spectrometer system planned for Hall $\mathrm{C}$ has been approved. Pions will be observed in the short-orbit spectrometer (SOS) which will serve as the second arm. The ANL medium-energy physics group has assumed responsibility for the construction and initial operation of the SOS.

*Rutgers University, †University of Colorado, †CEBAF, §CEN Saclay, France, INorthwestern University

$\ell$. The Energy Dependence of Nucleon Propagation in Nuclei as Measured in the (e,e'p) Reaction (D. F. Geesaman, R. J. Holt, H. E. Jackson, C. E. Jones, S. Kaufman, D. Potterveld, J. P. Schiffer, B. Zeidman, D. Dutta,* R. E. Segel,* E. R. Kinney, † D. van Westrum, † B. W. Filippone, $\neq$ R. D. McKeown, $\S$ R. Milner,$\S$ and R. Gilman ()

A proposal was approved by the CEBAF PAC-5 to continue (e,e'p) studies of proton propagation in nuclei for protons in the energy range of $400-2000 \mathrm{MeV}$. In this energy range the nature of the $\mathrm{N}-\mathrm{N}$ interaction changes from elastic to highly inelastic once the pion-production threshold is crossed. The theoretical description of proton propagation also changes considerably from nonrelativistic optical potentials to relativistic potentials to Glauber models. Information on proton propagation in this energy range is quite important to the CEBAF coincidence program. At higher energies, this reaction is used to study manifestations of more exotic mechanisms, such as increased transparency for hard collisions - color transparency.

*Northwestern University, †University of Colorado, $\ddagger$ California Institute of Technology, §Massachusetts Institute of Technology, TRutgers University 
The experiment will be carried out in the CEBAF Hall $\mathrm{C}$ using the HMS 6-GeV spectrometer and the SOS 1.8-GeV spectrometer. Electrons in the quasifree region will be detected in coincidence with protons with missing energies of less than $100 \mathrm{MeV}$. At two proton energies, $\mathrm{Tp}=400 \mathrm{MeV}$ and $\mathrm{Tp}=1000 \mathrm{MeV}$, Rosenbluth separations will be performed to study the A dependence of the longitudinal and transverse coincidence response independently. Since this proposal concentrates on the quasifree region, the projected count rates are relatively high and the background rates are calculated to be quite low. The collaboration developing the equipment for Hall $\mathrm{C}$ has chosen this experiment to be the tune-up experiment and the first experiment to be performed at CEBAF.

m. Measurement of Proton Polarization in the $d(\gamma, p) n$ Reaction (R. J. Holt,

S. J. Freedman, D. F. Geesaman, H. E. Jackson, C. E. Jones, D. Krakauer,

D. H. Potterveld, B. Zeidman, K. P. Coulter, * E. R. Kinney, † E. Beise, $\ddagger$ B. Filippone, $\ddagger$

R.D. McKeown, $\ddagger$ J. Napolitano,§ R. Milner,\$ R. E. Segel,I! D. Beatty, ** R. Gilman,**

C. Glashausser, ${ }^{* *}$ G. Kumbartzki, ${ }^{* *}$ R. Ransome, ${ }^{* *}$ Z.-E. Meziani, †† and D. Beck $\left.\ddagger\right)$

A proposal was approved by CEBAF PAC7 to measure angular distributions of the proton polarization for the $\mathrm{d}(\gamma, \overline{\mathrm{p}}) \mathrm{n}$ reaction in the $\mathrm{GeV}$ region. This pronosed measurement will test the validity of extensions of conventional nuclear-physics theories to the higher energy regime. The results of the experiment will further constrain the evidence from SLAC experiments NE8 and $\mathrm{NE} 17$ that asymptotic scaling has been observed above a photon energy of $1.3 \mathrm{GeV}$. Photoproton polarization measurements at lower energy indicate that the magnitude of the polarization increases with energy. This is consistent with the observation that polarizations are large in high-energy processes, e.g. $A_{n n}$ in $p p \rightarrow p p$ scattering or $A_{y}$ in $p p \rightarrow \pi^{0} X$. However, the polarizations in hadron-hadron scattering are believed to arise from Landshoff mechanisms. The higher energy photoproton experiment will permit the first measurements of polarization for a reaction, $\gamma \mathrm{d} \rightarrow \overrightarrow{\mathrm{p}} \mathrm{n}$, where there are no Landshoff terms. The experiment would make use of a polarimeter installed in? either the High Resolution Spectrometer in Hall A or the Short Orbit Spectrometer in Hall C at CEBAF.

\begin{abstract}
*University of Michigan, †University of Colorado, $\ddagger$ California Institute of Technology, §Rensselaer Folytechnic Institute, TMassachusetts Institute of Technology, IINorthwestern University, $* *$ Rutgers University, $\dagger+$ Stanford University, $\ddagger \ddagger$ University of Illinois
\end{abstract}

n. Short-Orbit Spectrometer for Hall C (H. E. Jackson, D. Potterveld, and B. Zeidman)

An examination of the proposed experimental program for Hall $\mathrm{C}$ at CEBAF reveals a major emphasis on coincidence experiments involving a "core" spectrometer and a second arm capable of detecting particles with momenta $<2 \mathrm{GeV} / \mathrm{c}$ with moderate energy and angular resolution. In most cases, the core spectrometer serves to tag a virtual photon, which induces a reaction in a nuclear target resulting in the sjection of a hadron in the energy range $(0.2-2.0 \mathrm{GeV})$ which is observed in the second spectrometer. Nuclear physics topics addressed in these experiments include color transparency, nucleon propagation, pion electroproduction, and hyperon physics. All of these programs require an acceptance in the hadron spectrometer as large as possible in solid angle and momentum to maximize operational efficiency. In addition, relatively short spectrometer drift lengths are required in experiments involving detection of pions or kaons in order to minimize decay losses. Because the requirements for energy resolution in this class of experiments is moderate, typically $\sim 10^{-3}$, an optimized design with a short optical length less than $10 \mathrm{~m}$ will provide a well-matched spectrometer capability. Excellent particle discrimination will be essential for detection of pions and kaons in th $;$ presence of high backgrounds. Operation at luminosities as high as $10^{38} / \mathrm{cm}^{2} \mathrm{sec}$ will be required frequently. To provide this second-arm capability we are building, under contract to CEBAF, a short-orbit spectrometer, the SOS, based on a QDD design which has been developed recently at the Los Alamos Meson Physics Facility. The QD 
configuration provides a large momentum acceptance, with good energy resolution and solid-angle acceptance in a very compact geometry which can meet the needs of a broad spectrum of studies appropriate for Hall $\mathrm{C}$ at CEBAF. The spectrometer pair system is illustrated in Fig. III-5.

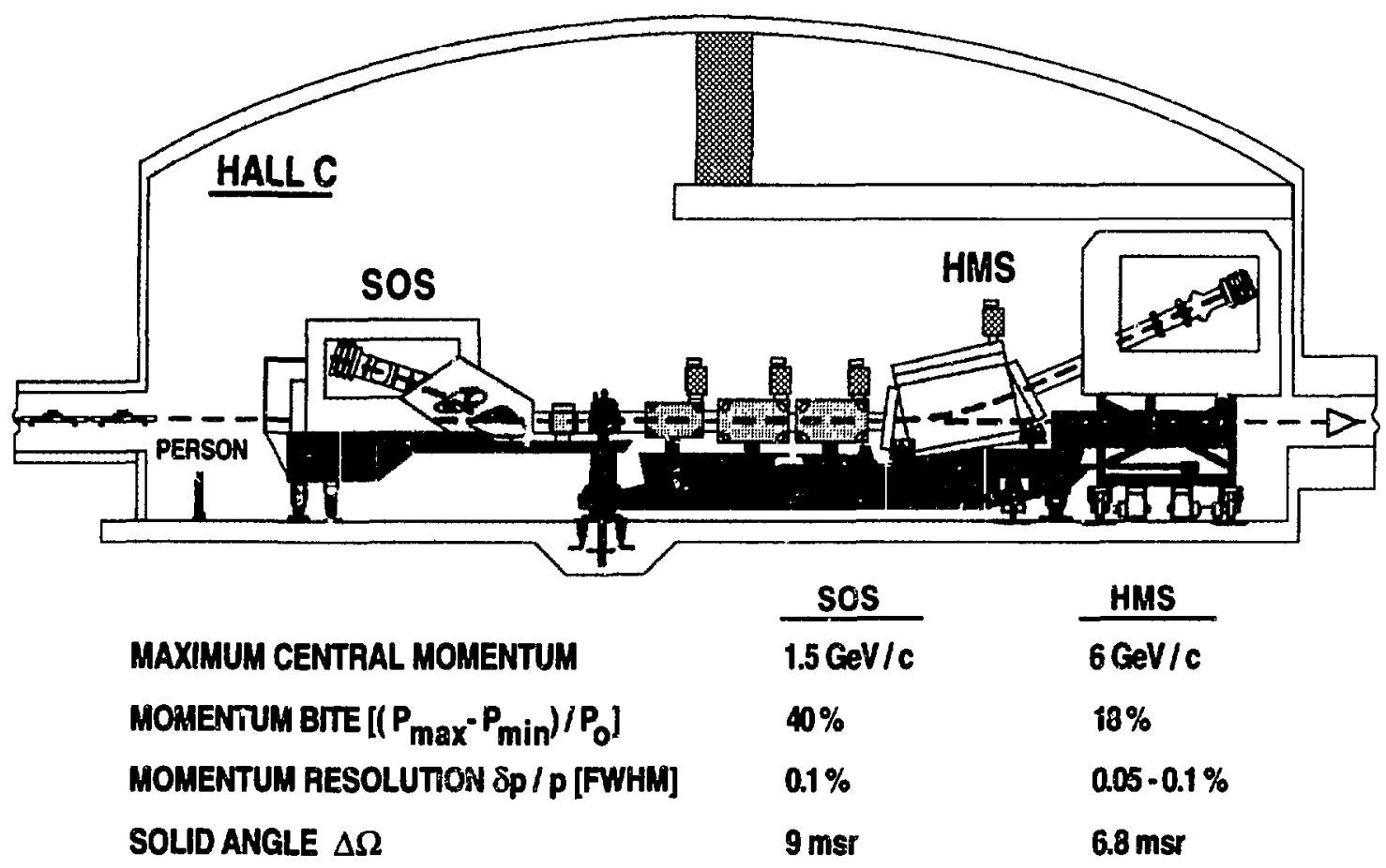

Fig. III-5. Schematic representation of the two spectrometers, the SOS and the HMS to perform the initial coincidence experiments at CEBAF.

The optical design is point-to-point in both the dispersive (vertical) and the transverse (scattering) planes. For a 1-mm target spot, the first-order resolving power is approximately 2200 , while the angular resolution is $<5 \mathrm{mr}$. Because of the reverse bend in the second dipole, there is a relatively small net deflection of the beam through the spectrometer, a property particularly useful for polarization measurements. Because of the strong edge focusing, the optical length of the spectrometer is only $\sim 7.4$ meters. The rigid structural design, coupled with a compact focal-plane detector package, yields a device that is readily adapted to out-of-plane measurements. SOS has been designed with a spherical bearing system at the spectrometer pivot and a hydraulic lifting assembly at the rear of the spectrometer carriage which allows the system to operate up to $20^{\circ}$ above the horizontal plane. All of the major components of the spectrometer have been fabricated and delivered to CEBAF. The dipole magnet has been assembled and field mapping is scheduled for January 1994. Complete assembly of the carriage and shield house are scheduled for early 1994 with commissioning to begin during the summer using the first beams available at CEBAF in Hall C. Operation of SOS in conjunction with the High Momentum Spectrometer in Hall C will provide a coincidence capability and will serve as a general-purpose second arm in a wide variety of experiments planned at CEBAF. 
o. Proposal to Measure Spin-Structure Functions and Semi-Exclusive Asymmetries for the Proton and Neutron at HERA (K. Coulter, D. F. Geesaman, R. J. Holt, H. E. Jackson, C. E. Jones, V. Papavassiliou, M. Poelker, D. Potterveld, L. Young, B. Zeidman, P. Green,* G. Greeniaus,* Y. Ke,* P. Kitching,* N. Rodning,* E. J. Beise, $\dagger$ B. F. Filippone, $\uparrow$ T. Gentile,$\dagger$ A. Lung, $\dagger$ R. D. McKeown, $\dagger$ M. Pitt,$\dagger$

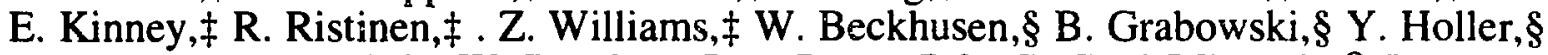

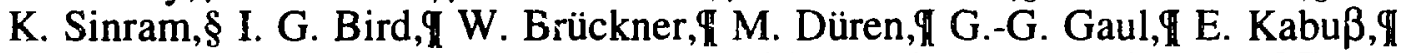
B. Martin, $\mathbb{T}$ D. Nowotny, $\mathbb{T}$ B. Povh, $\mathbb{T}$ K. Rith, $\mathbb{T}$ C. Scholz, T T. Shibata, $\mathbb{T}$ E. Steffens, $\mathbb{T}$ H. Vogt, I K. Zapfe, I F. Zetsche, I D. H. Beck, II R. Laszewski,\| C. N. Fapanicolas, II S. E. Williamson,ll H. J. Bulten,** J.F.J. van den Brand,** W. Haeberli, ${ }^{* *}$ S. Price, ${ }^{* *}$ T. Wise, ${ }^{* *}$ D. Fick, $\dagger \dagger$ W. Luck, $\dagger \dagger$ F. Rathmann, $\dagger \dagger$ R. Ent, $\ddagger \ddagger$ J.-O. Hansen,,$\ddagger$

L. Kramer, $\ddagger \ddagger$ K. Lee, $\neq \ddagger$ R. Milner, $\ddagger$ R. Redwine, $\ddagger \ddagger$ N. Simicevic, $\ddagger \ddagger$ B. Braun, $\S \S$

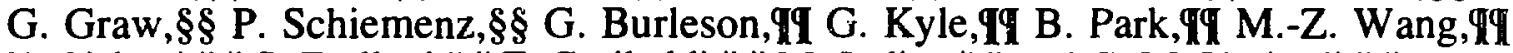
E. Cisbani, || || S. Frullani, II || F. Garibaldi,\| || M. Jodice, II I| and G. M. Urciuoli || II)

Nucleon spin physics will be studied in the HERMES experiment, which will use polarized internal targets of essentially pure atomic $\mathrm{H}, \mathrm{D}$. and ${ }^{3} \mathrm{H}$ ie in the HERA electron storage ring at DESY. A series of measurements of spin-dependent properties of the nucleon and few-body nuclei will be made; the spin structure function $\mathrm{g}_{1}(\mathrm{x})$ of the proton and neutron will be measured to test the Bjorken sum rule and study the fraction of the nucleon spin carried by quarks; the spin structure function $g_{2}(x)$, sensitive to quark-gluon correlations, and the structure functions $b_{l}(x)$,

\footnotetext{
*University of Alberta, Canada, $\nmid$ California Institute of Technology, $\ddagger$ University of Colorado, $\S$ Deutsches Elekı;onen-Synchrotron, Hamburg, Germany, TMax-Planck Institut für Kernphysik, Germany, IIUniversity of Illinois, **University of Wisconsin, ††University of Marburg, Germany, $\ddagger$ Massachusetts Institute of Technology $\S$ University of München, Germany, TTNew Mexico State University II IINFN Sezione Sanita, Italy
}

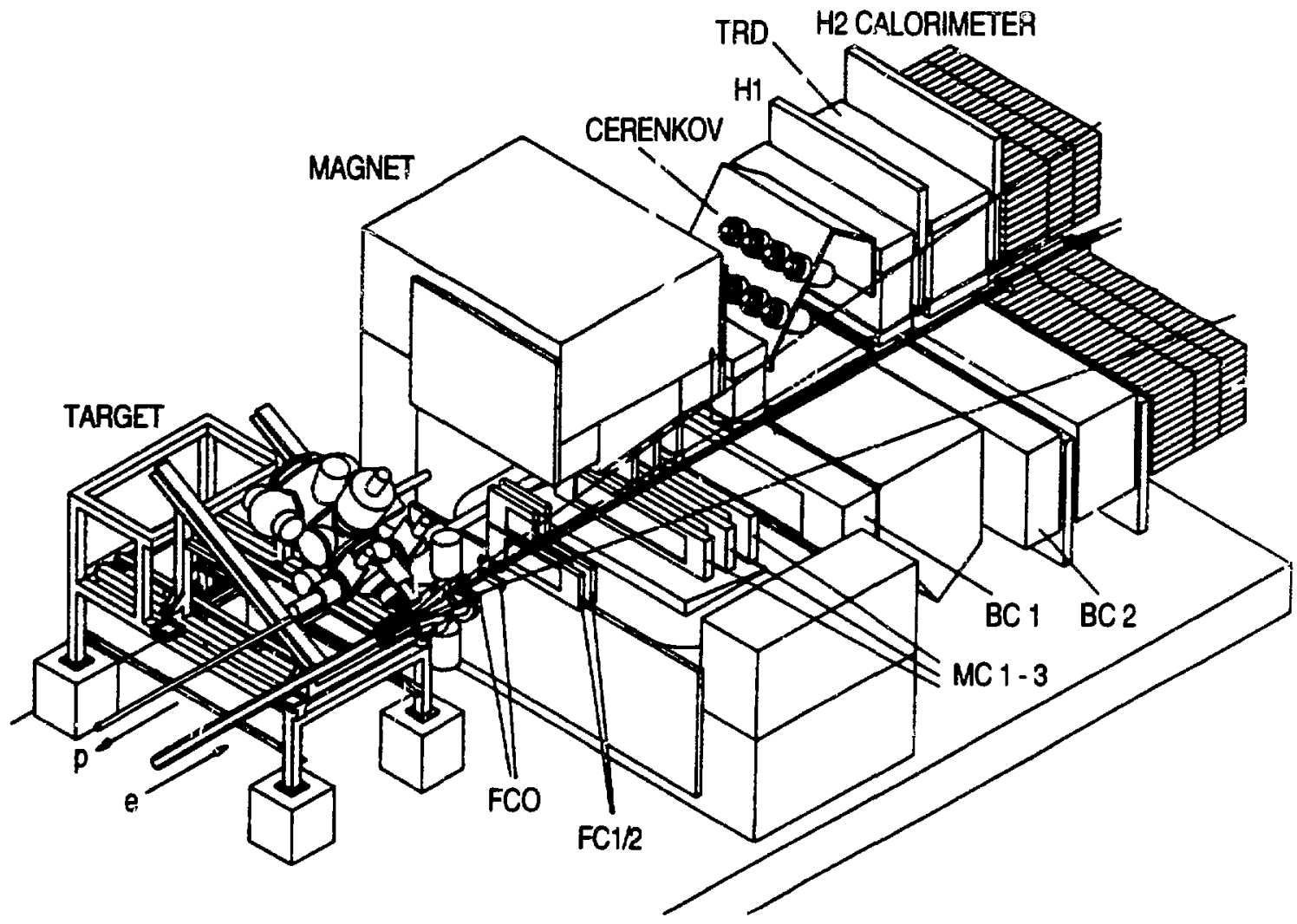

Fig. III-6. Schematic view of the HERMES magnetic spectrometer. 
and $\Delta(x)$, sensitive to nuclear binding effects, will be measured; and, using the particle identification capability of the HERMES detector, pions will be detected in coincidence with the scattered electrons. The coincident hadron measurements represent the most important extension that can be made at this time to the existingmeasurements on the nucleon spin structure functions because they provide information about the flavor-dependence of the quark spin distribution in the nucleon. A schematic view of the HEKMES spectrometer is presented in Fig. III-6 Argonne is providing the Cerenkov counter to be used for particle identification and developing the drifilm coating technique for the ultrathin target cell required for this experiment. The HERMES collaboration intends to use polarized targets with the highest available figures of merit, and the Argonne laser-driven source offers the most promise for a significant advance in present-day targets.

p. Measurement of $\overline{\mathbf{d}} / \overline{\mathbf{u}}$ in the Nucleon (D. F. Geesanan, H. E. Jackson, S. Kaufman, V. Papavassiliou, B. Zeidman, D. Isenhower,* M. Sadler,* Y. C. Chen, $\dagger$ G. C. Kiang, $\dagger$

P. K. Teng, $\dagger$ M. J. Wang, $\dagger$ B. Filippone $\ddagger$ R. McKeown, $\ddagger$ C. N. Brown,,

W. E. Cooper,\$ C. S. Mishra,\$ T. A. Carey, I G. T. Garvey,I D. M. Jansen, 1

M. J. Leitch,I J. B. McClelland,I P. L. McGaughey,I C. L. Morris, I J. M. Moss, I

J. C. Peng,I D. M. Kaplan,II T. C. Awes, ${ }^{* *}$ F. E. Obenshain, ** G. R. Young,;*

F. Z. Plasil, ** Hee Kim,** S. Saini,** P. Stankı's,** S. P. Sorenson, $\dagger \dagger$

C. A Gagliardi, $\ddagger \ddagger$ and R. E. Tribble $\ddagger$ )

Recent experimental results on the deep inelastic structure functions on hydrogen and deuterium, combined in a sum rule analysis, suggest that there is a suistantial dirference between the $\bar{u}$ and $\bar{d}$ sea in the proton. The Drell-Yan process where a quark from a projectile annihilates with an antiquark in the target to form a timelike-virtual-photon can provide a direct measurement of the $x$ dependence of the antiquark distributions. A new experiment, E866, has been approved for the next fixed target cycle at FNAL to measure accurately the Drell-Yan production of di-muon pairs using an 800-GeV proton beam on liquid hydrogen and deuterium targets with relative systematic errors of $\sim 1 \%$. This will measure $\bar{d} / \overline{\mathrm{u}}$ to $1 \%$ accuracy for $0.05<x<0.15$ and with less statistical accuracy out to $\mathrm{x} \sim 0.3$. High statistics $\mathrm{J} / \psi$, and $\psi^{\prime}$ production data will also be obtained.

It is expected that the experiment will take data in FY 1996.

*Abilene Christian University, †Academia Sinica, Taiwan, ROC, $¥$ Carifornia Institute of Technology, §Fermi National Accelerator Laboratory, ILos Alamos National Laboratory IINorthern Illinois University, **Oak Ridge National Laboratory, ††University of Tennessee $\ddagger \ddagger$ Texas A\&M University 


\section{THEORETICAL PHYSICS}

The Argonne Theory Program addresses issues concerning the dynamics of hadrons and nuclei. The forus of our recent investigation is on a broad range of theoretical questions raised by the new facilities employing electrons and heavy ions. The theoretical tasks involve the construction of models appropriate to various kinematic regions and the resolution of problems arising from the relations of the models to each other and to fundamental theory. The principal areas of our program are nuclear dynamics with subnucleon degrees of frecdom, nuclear forces and nuclear many-body problems, nuclear structure, and heavy-ion interaciions. Our objectives are: 1) to investigate the roles of mesons, nucleon resonances and quark-gluon degrees of freedom in determining hadronic and nuclear dynamics, 2) to understand the stability, structure, and response of nuclei in terms of interactions between individual nucleons, and 3 ) to investigate heavy-ion reaction mechanisms and to understand nuclear structure in terms of collective degrees of freedom and effective interactions. Most research projects involve collaborators at U.S. and foreign universities and research institutes. We stress the importance of interactions with experimental efforts at Argonne and major facilities around the world.

\section{A. NUCLEAR DYNAMICS WITH SUB-NUCLEONIC DEGREES OF FREEDOM}

The objective of this research program is to investigate the roles of mesons, nucleon resonances, and quark-gluon degrees of freedom in determining nuclear dynamics.

Our investigation of intermediate energy reactions is based on a meson-exchange Hamiltonian model which has been developed over the years by carrying out extensive studies of $\pi N, \gamma \mathrm{N}, \mathrm{NN}$, and $\pi \mathrm{d}$ reactions. Recently we have improved the dynamical content of the model by extending the non-resonant $N N \leftrightarrow \pi N N$ pion production operator to include the short-range mechanisms as implied by the nucleon-nucleon interaction. The constructed model has resolved the problems of describing threshold $\mathrm{pp} \rightarrow \mathrm{pp} \pi^{0}$ data and the recent ${ }^{3} \mathrm{He}\left(\pi^{-}, \mathrm{nn}\right){ }^{3} \mathrm{H}\left(\pi^{-}, \mathrm{np}\right)$ data with stopped pions. We have applied the model to $\left(\epsilon, e^{\prime}\right),\left(\pi, \pi^{\prime}\right)$ and $(\gamma, \pi)$ reactions on lp-shell nuclei. The model has also been used to calculate the properties of a pion gas at finite temperature and medium effects on $\Delta$ propagation and $N N$ and $N \Delta$ collisions, which are theoretical input to various relativistic heavy-ion calculations. To address physics at CEBAF, we are continuing our effort to extend the model to include higher mass nucleon resonances $\left(\mathrm{N}^{*}\right)$ and the production of heavy mesons.

We performed a chiral perturbation theory calculation of $\mathrm{p}\left(\mathrm{e}, \mathrm{e}^{\prime} \pi\right)$ near threshold. It is found that the recent $p\left(e, e^{\prime} \pi^{0}\right)$ data are consistent with the low energy dynamics dictated by spontaneously broken chiral symmetry.

We are continuing our effort to develop the Dyson-Schwinger equation (DSE) approach to quantum chromodynamics (QCD), which is an alternative to lattice gauge theory in describing nonperturbative dynamics. A QCD-based model field theory, the Global Color-symmetry Model (GCM), was developed and improved by using a confining ansatz for the form of the effective gluon propagator. The GCM was used to describe successfully the electromagnetic pion form factor at space-like momentum-transfer and $\pi-\pi$ scattering up to about $600 \mathrm{MeV}$. We are currently solving covariant Faddeev equations for the hadronic Faddeev amplitudes as a step to a full understanding of the $\rho-\omega$ mixing contribution to charge symmetry breaking in the nucleonnucleon interaction.

We continue to study electron scattering from few-nucleon systems within the framework of relativistic Hamiltonian particle dynamics. We found that our approach can be related to Lagrangian field theory in the Euclidean space-time formulations of quantum field 
theories, which provide the mathematical basis for nonperturbative approximations, and for precisely defined models of finite systems. We expect to use this approach for the construction of current operators that are consistent with Hamiltonian particle dynamics.

Studies on relativistic mean field theories and quantum electrodyı amics (QED) were also done.

\section{a. Hadronic and Electromagnetic Production of Mesons and Nucleon Resonances at GeV Energies (T.-S. H. Lee, M. Pichowsky, K. A. Bugaev,* S. N. Yang, † and F. Tabakin $\ddagger$ )}

The Hamiltonian formulacion, developed by Nozawa, Blankleider and Lee (NBL), was extended to investigate the electromagnetic excitations of nucleon resonances $\left(\mathrm{N}^{*}\right)$ at energies accessible to CEBAF. Motivated by the existing QCD-based hadron models, we assume that the basic resonant interaction mechanisms of the model Hamiltonian are the absorption and emissi un of photons and mesons by a bare quark core. The matrix elements of nonresonant interactions are deduced from low-order Feynman diagrams of an effective Lagrangian with chiral symmetry. The standard projection operator technique was applied to obtain a set of unitary scattering equations for describing $\pi \mathrm{N}$ and $\gamma \mathrm{N}$ reactions up to the GeV energy region. By assuming that the nonresonant two-pion continuum can he approximated as a fictitious $\sigma \mathrm{N}$ state, the scattering equations can then be cast into a set of coupled-channels equations involving only two-particle $\gamma N, \pi N, \eta N, \rho N, \pi \Delta, \omega N$ and $\sigma N$ channels, which can be solved by well-developed numerical methods. The bare coupling constants and the range parameters of the hadronic form factors are adjusted to reproduce $\pi \mathrm{N}$ scattering phase shifts up to $2-\mathrm{GeV}$ incident pion energy. We then explore the dependence of the $\gamma \mathrm{N} \rightarrow \pi \mathrm{N}$ and $\mathrm{N}\left(\mathrm{e}, \mathrm{e}^{\prime} \pi\right)$ observables on the $\gamma \mathrm{N} \rightarrow \mathrm{N}^{*}$ excitation strengths predicted by various QCD-based models of hadrons.

In the $\Delta$-excitation region, the model is reduced to the NBL . lodel, which was very successful in describing extensive resuits of $\gamma N \rightarrow \pi N$ and $N\left(e, e^{\prime} \pi\right)$ data. In the past two years, we applied the approach to investigate the p-wave Roper $N^{*}(1440)$ resonance. It was found that the $\pi N_{\text {phase- }}$ shift data in the $P_{11}$ channel favors a bare mass of about $2000 \mathrm{MeV}$ for the Roper resonance. This corresponds to a small radius of the quark core within the cloudy bag model of hadrons. The focus of our current effort is the excitation of $\mathrm{N}^{*}$ resonances as predicted by the constituent quark model and the $N\left(e, e^{\prime}\right)$ spin observables associated with the vector-meson production.

\footnotetext{
*University of Hannover, Germany, †National Taiwan University, ROC, $\ddagger$ University of Pittsburgh
}

\section{b. Chiral Symmetry and Electroproduction of Pions at Threshold in Chiral Perturbation Theory (T.-S. H. Lee, V. Bernard,* N. Kaiser, † and Ulf-G. Meissner $\ddagger$ )}

The electroproduction of pions off protons ciose to threshold is studied within the framework of baryon chiral perturbation theory. The approach is based on the fundamental QCD property that at low energies the strong interactions are dictated by the spontaneously broken chiral symmetry. The calculation has been done up to the 1-loop level by carrying out order-by-order renormalization procedures. A thorough study of thelow-energy theorems related to electroproduction of pions was carried out. Our study showed how the axial radius of thenucleon can be related to the $S$-wave multipoles $E_{0+}^{(-)}$and $\mathrm{L}_{0+}^{(-)}$. The chiral perturbation theory calculations of the $\mathrm{g}^{*} \mathrm{p} \circledast \mathrm{p}^{0} \mathrm{p}$ reaction near threshold were found to be in good agreement with the recent NIKHEF data. We also investigated the influence of some isospin-breaking effects. For future experimental tests of the underlying chiral dynamics, extensive predictions of differential cross sections and multipole amplitudes were made.

\footnotetext{
*Université Louis Pasteur, France, †Technische Universität München, Germany, $\ddagger$ Universität Bern, Switzerland
} 
c. Short-Range Exchange Contributions to Pion Absorption on Nuclei (T.-S. H. Lee, L. L. Kiang, * and D. O. Riska†)

By a direct extension of Weinberg's effective pion-nucleon chiral Lagrangian to nuclei, the nuclear S-wave pion production operator is described by the axial charge density operator of the two-nucleon system. It is shown that the short-range axial exchange charge operator implied by the nucleon-nucleon interaction enhances the predicted cross section for the reaction $\mathrm{pp} \rightarrow \mathrm{pp} \pi^{0}$ near threshold by factors of 3-5. This explains most of the under-prediction obtained with the single-nucizon operator and the conventional $S$-wave pion rescattering operator. The result implies that the cross section for $\mathrm{pp} \rightarrow \mathrm{pp} \pi^{0}$ can provide direct information on the short-range components of the nucleon-nucleon interaction. This work was published in Phys. Rev. Lett. 7U, 2237 (1993). The model was applied to study the absorption of stopped pions by ${ }^{3} \mathrm{He}$. We found that the short-range mechanism brings the calculated ratio ${ }^{3} \mathrm{He}\left(\pi^{-}, \mathrm{nn}\right) /{ }^{3} \mathrm{He}\left(\pi^{-}, \mathrm{np}\right)$ from a value of 200 to 5 . This is in excellent agreement with the data. We are extending the approach to derive three-nucleon absorption mechanisms from the existing models of three-nucleon forces.

*National Tsing-Hua Unıversity, Taiwan, ROC, †University of Helsinki, Finland

\section{d. Dependence of $p\left(e, e^{\prime} \pi\right)$ Cross Sections on the $\gamma N \leftrightarrow \Delta$ Fransition Form Factors (T.-S. H. Lee)}

The objective of this work is to predict the dependence of the p(e,e' $\pi$ ) cross sections on the $\gamma \mathrm{N} \leftrightarrow$ $\Delta$ transition form factors, which are of fundamental importance in testing QCD-based models of hadrons. Within the model of Nozawa, Blankleider and Lee, it is assumed that the nonresonant interactions can be described by the $\pi N$ scattering phase shifts and the low-order Feynman diagrams calculated from an effective Lagrangian with chiral symmetry. The only free parameters of the model are in the parameterization of the $G_{M}\left(q^{2}\right)$ for the magnetic $M 1, G_{E}\left(q^{2}\right)$ for the electric $E 2$, and $G_{C}\left(q^{2}\right)$ for the charge form factors of the $\gamma \mathrm{N} \leftrightarrow \Delta$ transition. It is found that the existing $\gamma \mathrm{N} \rightarrow \pi \mathrm{N}$ data can be best described by setting $\mathrm{G}_{\mathrm{M}}(0)=3.2$ and $\mathrm{G}_{\mathrm{E}}(0)=0.015$. With the $\gamma \mathrm{N} \leftrightarrow \Delta$ form factors parameterized by Jones, the predicted $p\left(e, e^{\prime} \pi\right)$ cross sections at $q^{2}$ $<1(\mathrm{GeV} / \mathrm{c})^{2}$ are in good agreement with the existing data. Significant deviations from the Jones form factors are needed to account for the SLAC data of $N\left(e, e^{\prime}\right)$ in the $\Delta$-excitation region, published recently. For future experimental tests, we made extensive predictions of various spin observables to be measured at MIT-Bates, Mainz, NIKHEF and CEBAF.

\section{e. Study of $\left(e, e^{\prime}\right),\left(\pi, \pi^{\prime}\right)$ and $(\gamma, \pi)$ Reactions on Nuclei (T.-S. H. Lee, N. Odagawa,* H. Ohtsubo, ${ }^{*}$ and T. Sato*)}

Theoretical approaches for investigating nuclear structure with $\left(e, e^{\prime}\right),\left(\pi, \pi^{\prime}\right)$ and $(\gamma, \pi)$ reactions were developed and applied to study the shell-model description of $10 \mathrm{~B}$. The distorted wave impulse approximation formulated in momentum space is used to calculate the cross sections of $\left(\pi, \pi^{\prime}\right)$ and $(\gamma, \pi)$ reactions from ihe $\pi N \rightarrow \pi N$ and $\gamma N \rightarrow \pi N$ off-shell amplitudes which are generated from the model of Nozawa, Blankleider and Lee. We found that the nonlocal effects due to $\pi \mathrm{N}$ off-shell dynamics and nucleon Fermi motion are important in predicting $(\gamma, \pi)$ cross sections. The one-pion-exchange two-body exchange currents are included in $\left(e, e^{\prime}\right)$ calculations. It was shown that core polarization effects, calculated in a perturbation approach including excitations up to $6 \hbar \omega$, are essential in obtaining quantitative agreements with the data with no adjustableparameters.

*Osaka University, Japan 

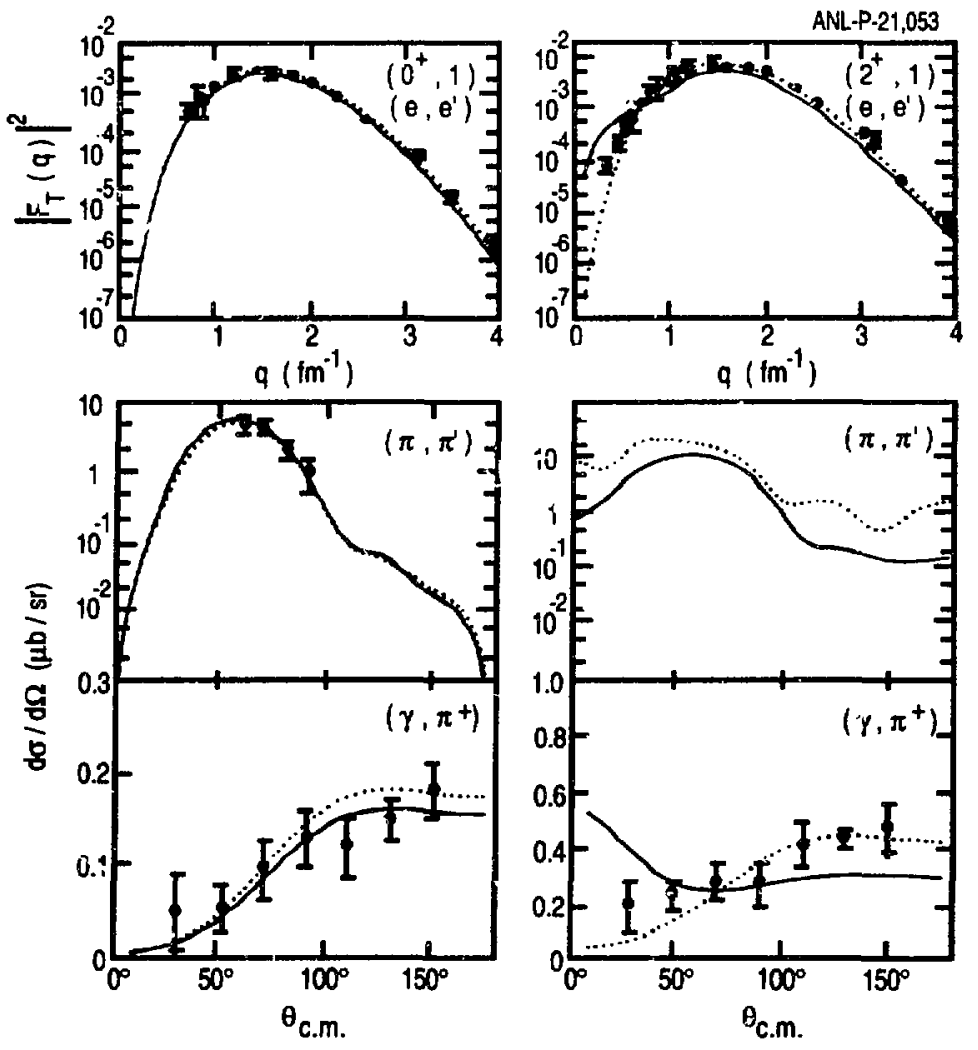

Fig. IV-1. The calculation for $\left(e, e^{\prime}\right),\left(\pi, \pi^{\prime}\right)$ at $E_{\pi}=164 \mathrm{MeV}$, and $\left(\gamma, \pi^{+}\right)$at $E_{\gamma}=183 \mathrm{MeV}$. The solid (dotted) curves are calculated using the shell model of Cohen and Kurath (Hauge and Maripuu).

In Figure IV-1 our predictions based on the shell-model of Cohen and Kurath (solid curves), and Hague and Maripuu (dotted curved), are compared in order to illustrate the use of $\left(e, e^{\prime}\right),(\pi, \pi)$ and $(\gamma, \pi)$ reactions in distinguishing nuclear structure theories which are almost equivalent in describing static properties of nuclei. Predictions for future $\left(e, e^{\prime}\right)$ and $(\gamma, \pi)$ experiments were also made. A paper describing our results was published in Phys. Rev. C 49.776 (1994).

\section{f. Electroproduction of Pions on the Deuteron and Three-Nucleon Systems} (T.-S. H. Lee, K. Bugaev.* P. U. Sauer*)

The electroproduction of pions on the deuieron and ${ }^{3} \mathrm{Fe}$ is being studied with the assumption that the basic pion production mechanisms can be described by the $N\left(e, e^{\prime} \pi\right)$ model developed by Nozawa and Lee. The objective is to find the kinematic regions where the reactions are sensitive to the electromagnetic form factors associated with pions, nucleons and $\Delta$ 's. The $\pi \mathrm{NN}$ final-state interactions in the $d\left(e, e^{\prime} \pi\right)$ reaction are calculated by solving the coupled $N N \oplus N \Delta \oplus \pi N N$ equations within the Argonne-Hannover unitary formulation. In ${ }^{3} \mathrm{He}\left(e, e^{\prime} \pi\right)$ calculations, the focus is on the calculation of the effects due to ihe $\Delta$ components in ${ }^{3} \mathrm{He}$, as suggested by Lipkin and Lee.

g. Spin Structure Functions of ${ }^{3} \mathrm{He}$ and the Deuteron (F. Coester, R. W. Schulze, ${ }^{*}$ and P. (I. Sauer*)

The spin-structure functions of ${ }^{3} \mathrm{He},{ }^{3} \mathrm{H}$ and the deuteron are related to the spin-structure functions of the proton and neutron, and to the target wave functions by well-defined

*University of Hannover, Germany 
convolution relations. There are four linear relations between the four spin structure functions of proton and neutron and the four spin structure functions of ${ }^{3} \mathrm{He}$ and ${ }^{3} \mathrm{H}$. We derived these relations in detail and numerical calculations were performed. The results of these calculations show that the relativistic effects are quite small.

\section{h. The Electromagnetic Current Operator and the Blankenbecler-Sugar Equations (F. Coester and D. O. Riska*)}

The effective electromagnetic current density for a two-nucleon system that is described by the Blankenbecler-Sugar equation was derived. In addition to the single-nucleon currents there are exchange currents of two different origins. The first is the exchange current required to compensate for the violation of the cortinuity equation in the impulse approximation. The second is an exchange current which arises in the quasipotential reduction from the BetheSalpeter equation, and which represents effects of suppressed degrees of freedom. Explicit general expressions are given for both of these exchange currents. The results are applicable to both elastic and inelastic processes.

*University of Helsinki, Finland

i. Nontrivial Vacuum Structure in Light-Front Hamiltonian Dynamics of Infinite Systems (F. Coester and W. N. Polyzou*)

Hamiltonian light-front dynamics of quantum fields may provide a useful approach to systematic non-perturbative approximations to quantum field theories. We investigated inequivalent Hilbert-space representations of the light-front field algebra in which the stability group of the light-front is implemented by unitary transformations. The Hilbert space representation of states is generated from the vacuum state by the operator algebra. There is a large class of vacuum states besides the Fock vacuum which meet all the invariance requirements. The light-front Hamiltonian must annihilate the vacuum and have a positive spectrum. Relations of the Hamiltonian to the nontrivial vacuum structure are exhibited in a paper pubished in Foundations of Physics 24, 387 (1994).

\section{j. $\quad$ Theory of Hadronic Models (F. Coester and W. N. Polyzou*)}

When a quantum field theory is specified by "Schwinger functions", which are moments of a Euclidean action, the Osterwalder-Schrader (OS) axioms specify conditions for the existence of local field operators in a Hilbert space of states. The key features in the relation of the Schwinger functions to quantum theory are: 1) the use of Euclidean time inversion to define an involution of test functions, and 2) reflection positivity of the Schwinger functions. The complete OS axioms, which are equivalent to the Wightman axioms, are too strong to be useful for the construction of nontrivial models. However a weaker set of requirements can be used to construct concrete models that combine the virtues of the familiar Green's function formalisms with the mathematical Hilbert-space structure required by quantum theory. The essential features are reflection positivity and the spectral condition which requires that the four-momenta of physical states are in the forward light cone. In this context we are investigating aigebraic and analytic properties of model Schwinger functions. This approach promises insight into the relations hetween hadronic models based on relativistic Hamiltonian dynamics on one hand and Bethe-Salpeter Green's function equations on the other. We expect this approach to lead to the construction of current operators that are consistent with Hamiltonian particle dynamics.

*University of Iowa 


\section{k. Gauge Covariance and the Fermion-Photon Vertex in Three- and Four- Dimensional, Massless Quantum Electrodynamics (C.D. Roberts and C. J. Burden*)}

The gauge covariance properties of three vertex ansätze in the Dyson-Schwinger equation for the fermion self energy were analyzed in three- and four-dimensional quantum electrodynamics. This study has important implications for the application of the nonperturbative DysonSchwinger equation approach to gauge field theories emphasizing as it does the manner in which gauge invariance can be incorporated. Based on the Cornwall-Jackiw-Tomboulis effective action, it was inferred that the spectral representation used for the vertex in the gauge technique cannot support dynamical chiral symmetry breaking. A criterion for establishing whether a given ansatz can confer gauge covariance upon the Dyson-Schwinger equation was presented and the Curtis-Pennington ansatz was shown to satisfy this constraint. An analytic solution of the Dyson-Schwinger equation for quenched, massless, three-dimensional quantum electrodynamics for arbitrary values of the gauge parameter in the absence of dynamical chiral symmetry breaking was obtained. A paper describing this work was published in Phys. Rev. C 47, 5581 (1993).

*Australian National University, Canberra, Australia

\section{$\ell$. $\pi-\pi$ Scattering in a QCD-Based Model Field Theory (C. D. Roberts, R. T. Cahill,*} N. Iannella, ${ }^{*}$ and M. E. Sevior $\dagger$ )

Using a model field theory, the Global Color-symmetry Model (GCM), which incorporates many of the important properties of quantum chromodynamics, such as quark confinement, dynamically broken chiral symmetry and asymptotic freedom, we calculated the kinematic and dynamical properties of pions; i.e., $\mathrm{f}_{\pi}, \mathrm{r}_{\pi}, \mathrm{m}_{\pi}$ and the scattering lengths and partial wave amplitudes in $\pi-\pi$ scattering. As the meson most important in nuclear dynamics, it is very important to understand the pion within the context of QCD. The version of the GCM used had three parameters and it was shown that an excellent description of pionic properties was possible in the model. This is illustrated in Fig. IV-2 where the solid line, which describes the data well up to $600 \mathrm{MeV}$, i.e., away from resonances, is our calculation and the dashed line is the result obtained using the Weinberg Lagrangian [which predicts $\mathrm{T}^{20}=0=\mathrm{T}^{22}$ ]. In the tree-level of the GCM approach, mesons are composite quark-antiquark bound states and their associated mesoncloud is a higher order effect. The results of this study support the point of view that this quark core is the dominant determining characteristic of hadronic properties and that the meson selfdressing and mixing is important only near resonances. A paper describing this work was published in Phys. Rev. D $\underline{49}, 125$ (1994).

*Flinders University of South Australia, Adelaide, †University of Melbourne, Australia 
Pi Pi SCATTERi:ivG

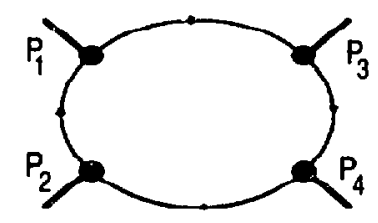

3-PARAMETER MODEL OF $\sigma_{v}$ and $\sigma_{\mathrm{s}}$ BASED ON DSE STUDIES

$\operatorname{Re}[100(E)]$

$\operatorname{Re}[\mathrm{TO2}(\mathrm{E})]$
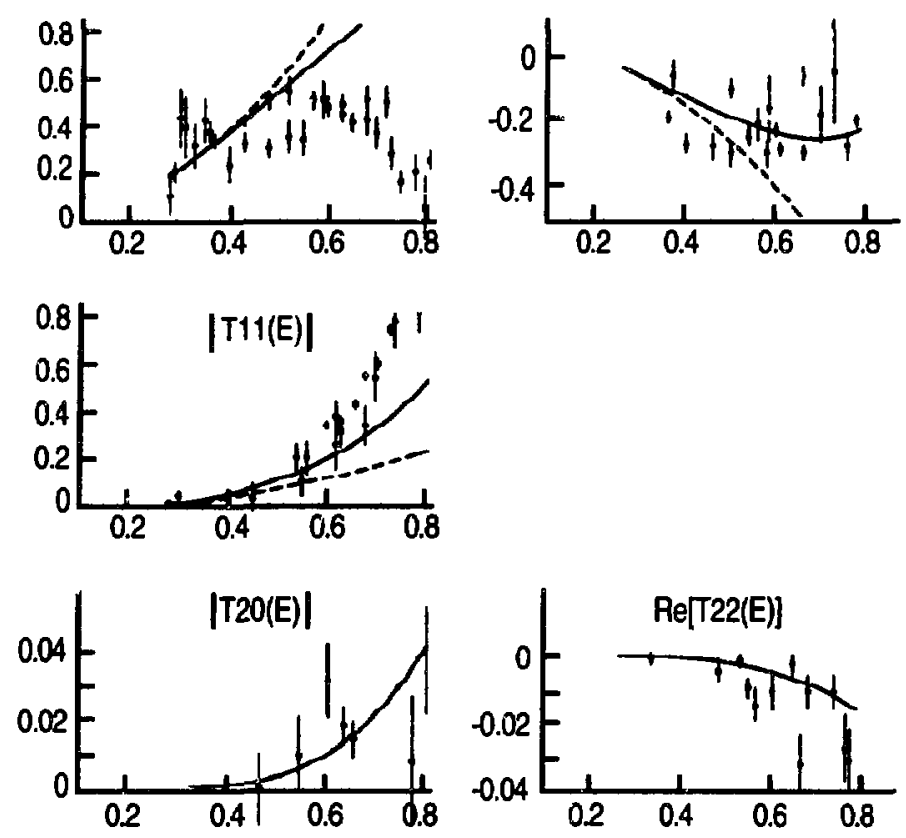

Fig. IV-2. The partial wave amplitudes for pion-pion scattering as calculated at tree-level in the Global, Colour-symmetry Model (solid line). The results obtained using Weinberg's effective Lagrangian (dashed line) are shown for comparison when they are non-zero. Our calculation describes the data well from threshold up to $600 \mathrm{MeV}$ where resonance contributions, which are absent at tree-level, become important.

m. $\rho \pi \pi$ and $\omega \pi \pi$ Vertex Functions Within a Confined-Quark Model (C. D. Roberts, K. L. Mitchell,* and P. C. Tandy*)

The $\rho \pi \pi$ and $\omega \pi \pi$ vertex functions were calculated in the Global Color-symmetry Model (GCM) using a confining quark propagator obtained in earlier studies. In studying the effect of charge-symmetry-breaking (CSB) in the $\mathrm{N}-\mathrm{N}$ potential, it is important to take into account the possible contribution of G-parity violating effects, such as $\omega \rightarrow \pi \pi$, which are allowed when there is a $u-d$ current-quark-mass difference. The three parameters in the version of the GCM were chosen to fit the kinematic and dynamical properties of the pion; i.e., $f_{\pi}, r_{\pi}, m_{\pi}$ and the scattering lengths and partial wave amplitudes in $\pi-\pi$ scattering. It was found that the G-parity violating $\omega \rightarrow \pi \pi$ vertex can be significant and may be an important part of the CSB piece of the N-N potential.

* Kent State University 
n. $\quad \rho-\omega$ Mixing Saif-Energy and Model Quark-Ghuon Dynamics (C. D. Roberts, K. L. Mitchell, ${ }^{*}$ P. C. Tandy, ${ }^{*}$ and R. T. Cahill†)

The contribution of the mixed u-d quark-locp to the Charge-Syrnmetry-Breaking (CSB) piece of the $\mathrm{N}-\mathrm{N}$ potential was studied in the Global Color-symmetry Model (GCM), using a confining quark propagator obtained in earlier studies. In fitting $\mathrm{N}-\mathrm{N}$ phase shifts it was found necessary to include a term in the $N-N$ potential that can be attributed to the rixing between $\omega$ alid $\rho$ mesons that arises because of isospin asymmetry at the quark level, as manifest in the small u-d currentquark-mass difference. To the present, this term was modeled and assumed to be momentum independent. It is important to understand this term in the context of QCD. The preliminary results of this study suggest that the quark loop can yield only $25 \%$ of the phenomenologically determined value of this mixing term. This leaves room for contributions from other isospinbreaking effects, such as $\omega \rightarrow \pi \pi \rightarrow \rho$, which are possible when there is a u-d current-quark-mass difference, and are present in the GCM, but which were not considered hitherto.

*Kent State University, †Flinders University of South Australia, Adelaide, Australia

o. Pion Loop Contribution to the Electromagnetic Pion Charge Radius (C. D. Roberts, R. Alkofer, * and A. Bender*)

There is a widely held misconception, based on a misinterpretation of the application of chiral perturbation theory, that the electromagnetic structure of the pion is dominated by the pion's own pion-cloud. To clarify this, the Global Color-symmetry Model (GCM) was used to calculate the electromagnetic charge radius of the pion. In this calculation the contributions from the quark core and pion loop were identified and compared. It was shown explicitly that the divergence of the charge radius in the chiral limit is due solely to the pion loop and that, at the physical value of the pion mass, this loop contributes less than $15 \%$ to $\left\langle\mathrm{r}_{\pi}^{2}\right\rangle$; i.e. the quark core is the dominant determining characteristic for the pion. This suggests that quark-baseri models rohich fail to reproduce the $\left(m_{\pi}\right)$ divergence of $\left(\mathrm{r}_{\pi}^{2}\right)$ may nevertheless incorporate the duminant ch aracteristic of the pion: its quark core. The results of this study lend further support to the contention that, away from resonances, the dominant determining characteristic of kinernatic and dynamical properties of hadrons is their quark core.

*University of Tübingen, Germany

\section{p. Electromagnetic Pion Form Factor (C. D. Roberts)}

A phenomenological Dyson-Schwinger--Bethe-Salpeter equation approach to QCD, formalized in terms of the Global Color-symmetry Model (GCM), was used to calculate the generalized impulse approximation contribution to the electromagnetic pion form factor at spacelike $\mathrm{q}^{2}$ in the domain $[0,10] \mathrm{GeV}^{2}$. In effective field theories this form factor is often understood as simply being due to Vector Meson Dominance (VMD); but this does not allow for a simple connection with QCD where the VMD contribution is of higher order than that of the quark core. In the GCM, the pion is. treated as a composite bound state of aconfined quark and antiquark interacting via vector boson exchange. A direct study of the quark core contribution in QCD can be made. Two versions of the GCM have been employed so far with the more realistic form, used with success to describe $\pi$ $\pi$ scattering, providing excellent agreement with the available experimental data. This supports the contention that, away from resonances, the quark core is the dominant determining characteristic of hadronic properties. The result obtained in the GCM should yield the perturbative QCD result at large spacelike $-q^{2}$. This appears to be the case numerically and an analytic investigation of this domain is currently underway. 
q. Dynamical Chiral Symmetry Breaking and Confinement With an InfraredVanishing Gluon Propagator (C. D. Roberts, F. T. Hawes, ${ }^{*}$ and A. G. Williams $\dagger$ )

A model Dyson-Schwinger equation for the guark propagator, constructed by using an ansatz for the gluon propagator of the form $D(q) \sim q^{2} /\left[\left(q^{2}\right)^{2}+b^{4}\right]$ and two ansätze for the quark-gluon vertex: the minimal Ball-Chiu and the modified form suggested by Curtis and Pennington, was studied. The goal is to determine whether such a form of the gluon propagator, which was siuggested by a number of authors and which recent lattice simulations of QCD suggest may be plausible, can support dynamical chiral symmetry breaking and ensure quark confinement. The form of the gluon propagator at small spacelike momenta is crucial to the nature of the strong interaction spectrum but is presently unknown and information gathered in such studies is invaluable in supporting or invalidating given hypotheses. It was found that there is a critical value of $b=b_{c}$ surh that the model does not support dynamical chiral symmetry breaking for $b>b_{c}$. Further, it was shown inat uiis form of gluon propagator cannot confine quarks. As a consequence this form represents a physically unreasonable model. A paper describing this work was published in Phys. Rev. D $\underline{49}, 4683$ (1994).

*Florida State University, $\nmid$ University of Adelaide, Australia

\section{r. Dyson-Schwinger Equations and Their Application to Hadronic Physics \\ (C. D. Roberts and A. G. Williams*)}

At the invitation of the editor of "Progress in Particle and Nuclear Physics", a review article which describes the present status of the application of Dyson-Schwinger equations to nonperturbative studies of quantum electrodynamics, in three and four dimensions, quantum chromodynamics and hadronic physics was written. This article was written with the aim of making this increasingly useful and efficacious nonperturbative approach accessible to a larger group of physicists and to encourage its further and wider application. The article was completed and published in Progress in Particle and Nuclear Physics, Vol. 33, pp. 477-575 (1994), ed. A. Fässler (Pergamon Press, Oxford, 1994).

*University of Adelaide, Australia

\section{s. Complex Singularities in the Quark Propagator (C. D. Roberts, and C. J. Burden*)}

The singularity structure of the solution of the Dyson-Schwinger equation for the quark propagator in QCD, obtained with various ansätze for the dressed quark-gluon vertex and the dressed gluon propagator, was studied. This has important implications for quark confinement in QCD. It is also crucial to understanding the connection between Minkowski- and Euclideanspace formulations of gauge field theory. The preliminary results indicate the presence of complex conjugate poles in the quark propagator when the rainbow approximation is used, consistent with the results of other studies. The next step is to determine how this structure depends on the nature of the quark-gluon vertex, which is known to be crucial to maintaining gauge covariance in the Dyson-Scwhinger equation approach and was shown to be responsible for the elimination of singularities in the quark propagator when an infrared dominant model is used for the dressed gluon propagator.

*Australian National University, Canberra, Australia 
t. Dyson-Schwinger Equation Study of the Effect of the Higgs-Mechanism on Dynamical Chiral Symmetry Breaking (C. D. Roberts and L. Smith*)

An approximate Dyson-Schwinger equation for the quark self-energy derived from the Lagrangian of the standard model, which involves the coupling of the quark to the Higgs boson as well as to the gluon, was solved. The Higgs coupling in the standard model is currently supposed to be the origin of the current-mass of all elementary particles while dynamically broken chiral symmetry, driven by quark self-interactions, is responsible for the low mass of the pion relative to other hadrons. An unsatisfactory aspect of the standard model, however, is that the strength of the Higgs-quark couplings is arbitrary. A study of the interrelationship between these two mass-generating mechanisms is therefore important, especially if it serves to constrain the Higgs coupling. The preliminary results indicate that allowing for the feedback between the Higgs and quark selfinteraction terms in the Dyson-Schwinger equation leads to a suppression of the quark condensate; i.e., that the Higgs coupling suppresses dynamical chiral symmetry breaking. It may be possible to use this to constrain the Higgs-quark couplings. Currently the nature of the approximations used and the sensitivity of the results to them is being studied in order to judge their reliability.

\section{*University of Kansas}

\section{u. QCD at Finite Temperature and Density (C. $\Gamma$. Roberts)}

An approximate Dyson-Schwinger equation for the quark self energy in QCD was solved at finite temperature. This allows for a study of the temperature dependence of hadron masses and couplings and also for an estimate of the temperature at which chiral symmetry restoration should occur in QCD, fundamental quantities that are relevant to current and pianned experimental activities. The preliminary results indicate that the chiral symmetry restoration temperature is approximately 200 $\mathrm{MeV}$, which is consistent with estimates from lattice simulations of QCD. In addition, the study suggests, among other things, that the $\rho \pi \pi$ coupling constant diverges at the chiral symmetry restoration temperature and hence that $\rho$ mesons are depleted in the hadron gas as the critical temperature is approached. This suggests that a clear signal of chiral symmetry restoration may be found in a study of di-lepton production since $\rho \rightarrow \mathrm{e}^{+} \mathrm{e}^{-}$will be suppressed. Currently a more realistic model for the gluon propagator and quark-gluon vertex is being studied in order to judge the reliability of these predictions. The extension to the case of finite valence quark density is also being undertaken.

\section{v. Medium Effects on $\Delta$-Propagation, $\mathbf{N N}$ and $\mathbf{N} \Delta$ Collisions (T.-S. H. Lee)}

By using the Bruckner-Hartree-Fock approximation, the medium effects on the $\Delta$-nucleus potential and the NN $\rightarrow \mathrm{NN}, \mathrm{NN} \leftrightarrow \mathrm{N} \Delta$ and $\mathrm{N} \Delta \leftrightarrow \mathrm{N} \Delta$ transitions have been calculated from the coupled $\mathrm{NN}$ $\oplus \mathrm{N} \Delta \oplus \pi \mathrm{NN}$ model developed at Argonne in the past decade. The predicted density-dependence and momentum-dependence are theoretical input to the calculations of pion production from relativistic heavy-ion collisions. A series of lectures on the model and the calculations were delivered at the University of Giessen, and is being prepared for publication. Our rest:lts will be used by the Giessen group in their relativistic heavy-ion collision calculations based on the Boltzmann-Uehling-Uhlenbeck equation. The objective is to explore the $\Delta$-dynamics in the relativistic heavy-ion collisions. 
w. Effective Potentials for an Interacting Hadron Gas (S. Landowne and T.-S. H. Lee)

A major goal of nuclear physics research is to produce a new phase of matter, the quark gluon plasma, using relativistic heavy-ion collisions. In order to learn about the plasma phase, it will be necessary to understand the hadronic phase into which it decays. A useful approach to this problern is to postulate the existence of a thicrmalized hadrun gas and study its properties. We calculated effective potentials for paticles propagating, in a thermalized hadron gas. We have so far considered systems of pions only and of pions with up to $30 \%$ admixtures of nucleons at temperatures of $T=100-200 \mathrm{MeV}$. The effective potentials are computed self-consistently by folding forward scattering amplitudes with thermal distributions of scattering states. The amplitudes are constructed from separable interactions which reproduce empirical phase shifts. The resulting potentials are of typical nuclear magnitudes and have non-trivial momentum dependencies. We plan to utilize these results and extensions of them in further calculations of hadron gas systems and their decay modes. We plan to investigate conditions under which pion condensation occurs.

\section{x. Excitation of Nucleon Resonances (D. Kurath)}

Matrix elements were calculated for the excitation of nucleon resonances $\left(\mathrm{N}^{*}\right)$ in $(\mathrm{N}, \gamma)$ and $(\mathrm{N}, \pi)$ reactions using the harmonic oscillator quark model. These are resonances with no strange quarks and excitation of one or two oscillator quanta. The $\mathrm{N}^{*} \rightarrow \gamma \mathrm{N}$ cases were calculated first and reproduced the published table of Koniuk and Isgur (one error was found). Speakez programs were written to carry out the numerical calculations. Some cases of the $\mathrm{N}^{*} \rightarrow \pi \mathrm{N}, \pi \Delta$ transitions were also evaluated. The results are being applied by T.-S. H. Lee and his collaborators in their study of $\mathrm{N}\left(\mathrm{e}, \mathrm{e}^{\prime} \pi\right)$ reactions.

\section{y. Relativistic Mean Field Theory of Nuclei (A. R. Bodmer)}

Previously we developed an extension of relativistic mean field theory which includes a quartic vector-meson self interaction. This leads to a one-parameter family of relativistic equations of state for nuclear matter consistent with the empirical properties of nuclei. We extended our model to nuclear matter with a large neutron excess, in particular, to neutron and neutron-star matter. The composition of the latter is determined by the equilibrium between neutrons, protons and electrons, with the neutrons being the dominant fraction. This work is being applied to obtain limits on the physically pernissible neutron star masses.

\section{z. Topological Effects in Quantum Mechanics (M. Peshkin and H. J. Lipkin*)}

The Aharonov-Bolim (AB) effect has attracted substantial interest because of features that have come to be called "topological". The motion of an electron is influenced by a stationary magnetic field from which the electron is forever rigorously excluded. For instance, the magnetic field is confined to the inside of an infinitely long cylinder and the electron is confined to the outside of the cylinder. The electron is subjected to the local gauge field $\mathbf{A}$ but not to the local Maxwell field B. The classical equations of motion, and therefore the operator equations of motion for the observables, contain no electromagnetic field, gauge or Maxwell. In the language of operator algebra, the effect exists because the multiply-connected topology of the domain of the electron's position $\mathbf{x}$ admits inequivalent representations of the commutation relation [x,mv] $=i$. In the language of Feynman diagrams, path integrals acquire a phase factor dependent only on their topological winding number even though no local Maxwell field appears in them. Why so-defined topological effects are deemed important is discussed elsewhere.

*Weizmann Institute of Science, Rehovot, Israel 
In recent ycars, two new phenomena, also said to be "topological", were suggested. In the Aharonov-Casher $(A C)$ effect, a neutron replaces the electron and an electrically charged wire along the $z$-axis replaces the magnetic field. A neutron moving in the $x y$-plane with its spin in the $\mathrm{z}$-direction experiences a motional magnetic field parallel to the spin direction but there is no force on the neutron because the spin variable $\sigma_{z}$ is a constant of the motion. In the scalar Aharonov-Bohm effect (SAB), it is the same except that the motional magnetic field is replaced by our external magnetic field in the laboratory frame. Then the $\sigma \cdot \mathbf{B}$ term in the Hamiltonian uses $\mathbf{B}$ in some sense as a potential. The operator equations of motion of the observables $\mathbf{x}$ and $\mathbf{v}$ do not contain the field $\mathbf{B}$, at least if that field is uniform, but there are nevertheless observable effects.

The equations of motion for the spin components $\sigma_{\mathrm{x}}$ and $\sigma_{\mathrm{y}}$ do contain the magnetic field, but those have vanishing expectation in an eigenstate of $\sigma_{z}$ and they do not appear in the Hamiltonian or in the equations of motion. For these reasons, many authors have discussed $A C$ and $\mathrm{SAB}$ effects as new topological phenomena of the same type as $\mathrm{AB}$ effect and experiments were carried out to demonstrate them.

We compared $\mathrm{AC}$ and $\mathrm{SAB}$ effects with $\mathrm{AB}$ effect and find that there is a great difference. The spin is a quantum mechanical variable which cannot be dismissed as a constant even in a state where its expectation value is constant. Moreover, we identified two spin correlation operators, $S(t)=(1 / 2)\left[\sigma_{x}(0) \sigma_{x}(t)+\sigma_{y}(0) \sigma_{y}(t)+h . c.\right]$ and $V(t)=(1 / 2)\left[\sigma_{x}(0) \sigma_{y}(t)-\sigma_{y}(0) \sigma_{x}(t)+h . c.\right]$, whose equations of motion do contain the local Maxwell field and which precess under the influence of a local torque in such a way as to bring about the observed effects. Our results agree cbjectively with everybody else's calculations and with the experiments. However, we account for the observed effects in the ordinary way without invoking any topology or nonlocality.

This work has been submitted to Physical Review Letters. It goes beyond earlier work by Peshkin and by others in that it challenges the interpretation of the neutron experiments even if those experiments should be done with polarized neutrons, and thereby it challenges the interpretation of the $\mathrm{AC}$ and $\mathrm{SAB}$ effects.

\section{B. NUCLEAR FORCES AND NUCLEAR SYSTEMS}

The goal of this program is to achieve a description of nuclear systems ranging in size from the deuteron to nuclear matter and neutron stars using a single parameterization of the nuclear forces. Aspects of our program include both the construction of realistic two- and three-nucleon potentials and the development of many-body techniques for computing nuclear properties with these interactions. Detailed quantitative and computationally-intensive studies are essential parts of this program.

A new nucleon-nucleon potential, Argonne $v_{18}$, was developed over the last two years. It includes a charge-independent piece similar to the older Argonne $v_{14}$ model, plus additional charge-dependent and charge-asymmetric terms. It fits the latest pp and np elastic scattering data with a $\chi^{2}$ of only 1.09 per degree of freedom, as well as low-energy nn scattering parameters and deuteron properties. We also constructed a three-nucleon potential to be used in conjunction with the $\mathrm{v}_{18}$ model that gives the correct ${ }^{3} \mathrm{H}$ and ${ }^{4} \mathrm{He}$ binding energies in exact calculations, and we are formulating a consistent set of electromagnetic current operators.

The many-body calculations at Argonne are based primarily on the variational method with correlated operator trial functions. Parameters in the trial functions are varied to minimize the energy, and these optimized variational wave functions are then used to study other nuclear properties. The calculations can be separated into three groups, according to the size of the 
system: (1) direct Monte Carlo integration for few-body $(A \leq 8)$ nuclei, (2) a cluster expansion with Monte Carlo integrations for larger $(8 \leq \mathrm{A} \leq 40)$ nuclei, and (3) a diagrammatic cluster expansion with integral equation summation methods for nuclear and neutron matter. Although the methods vary, the calculations are all linked by the same Hamiltonian and very similar trial functions.

Because minimization of the ground state energy is the key to determining the wave function, much of our past wort. was devoted to evaluating binding energies and searching for improvements in the variational wave functions. Energies in ${ }^{3} \mathrm{H}$ and ${ }^{4} \mathrm{He}$ that are $\sim 2 \%$ above the values from availatle exact methods were achieved. A very significant development in the last year was the conversion of our few-body and light nuclei programs to run on Argonne's new 128-processor IBM SPI parallel supercomputer at speeds $\sim 6$ billion floating point operations (GFLOPS) per second. This made extensive calculations of ${ }^{40} \mathrm{Ca}$ possibie for the first time, as well as much more thorough studies of $6 \mathrm{Li}$ and ${ }^{16} \mathrm{O}$. We are also working with collaborators from Urbana and Los Alamos on Green's function Monte Carlo calculations of ${ }^{6} \mathrm{Li}$ and ${ }^{6} \mathrm{He}$ which now seem feasible.

In FY 1994 considerable effort went into calculating other nuclear properties, in particular isovector spin response and electron scattering data. These studies included the Euclidean response and sums, and proton-proton correlations in few-body nuciei. Calculations of 160 (e,e'p) reactions are continuing. Work on hypernuclei from ${ }_{\Lambda}^{4} \mathrm{H}$ to ${ }_{\Lambda}^{17} \mathrm{O}$, along with ca!culations of single-particle levels in large hypernuclei, is also continuing.

a. New Argonne Nucleon-Nucleon Potentia! (R. B. Wiringa, V. G. J. Stoks, ${ }^{*}$ and R. Schiavilla†)

We constructed a new realistic NN potential with explicit isospin-breaking terms, which we designate Argonne $v_{18}$ for its eighteen operator components. The wodel has a chargeindependent part with fourteen operator components that is an updated version of the Argonne $\mathrm{v}_{14}$ model. The latter was our standard nonrelativistic NN potential for most of the last decade, its chief virtues being a good fit to the $1981 \mathrm{np}$ phase shifts and deuteron properties and a simple structure convenient for many-body calculations. The fourteen terms each contain a radial function $v_{p}\left(r_{12}\right)$ multiplied by an operator, the operators being $1, \sigma_{1} \cdot \sigma_{2}, S, L \cdot S, L^{2}, L^{2} \sigma_{1} \cdot \sigma_{2}$, and $(\mathbf{L} \cdot \mathbf{S})^{2}$, and each of these times $\tau_{1} \cdot \tau_{2}$.

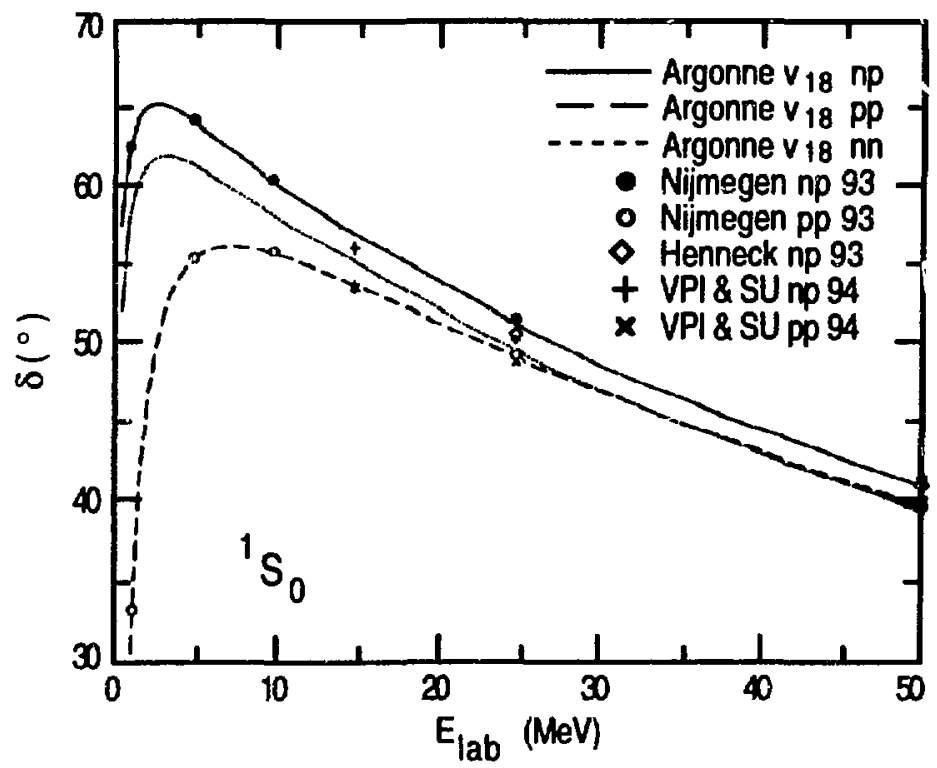

Fig. IV-3. Low-energy phase shifts in 'So for $n p, n n$, and pp scattering for Argonne $v_{18}$ potential, comrartio to different partial-wave analyses. 
Three charge-dependent and one charge-asymmetric operators are added in the new $v_{18}$ model so it can fit $\mathrm{pp}, \mathrm{np}$, and $\mathrm{nn}$ data simultaneously. The charge-dependent operators are obtained by multiplying the spin operators $1, \sigma_{1} \cdot \sigma_{2}$, and $S_{12}$ by the isotensor $T_{12}=3 \tau_{1 z} \tau_{2 z}-\tau_{1} \cdot \tau_{2}$, which differentiates between $\mathrm{np}$ and $\mathrm{pp}$ or $\mathrm{nn} \mathrm{T}=\mathrm{l}$ states. A major source of charge dependence in $\mathrm{NN}$ interactions is the mass difference of the charged and neutral pions, which is carefully treated in the new model. The charge-asymmetric operator is $\tau_{1 \mathrm{z}}+\tau_{2 \mathrm{z}}$ which splits pp and nn states; it is constrained by the difference between $\mathrm{nn}$ and pp scattering lengths. The low-energy ${ }^{1} \mathrm{~S}_{0}$ phase shifts for $\mathrm{pp}$, np and nn scattering are shown in Figure IV-3. The potential was fit directly to the Nijmegen pp and $\mathrm{np}$ scattering data base as well as the $\mathrm{nn}$ scattering length and deuteron binding energy. With 40 adjustable parameters it gives an excellent $\chi^{2} /$ degree of freedom of 1.09 for $4301 \mathrm{pp}$ and np data in the range $0-350 \mathrm{MeV}$. We are looking now to add a consistent set of two-body charge and current operators to calculate the deuteron electro-magnetic form factors.

*Flinders University of South Australia, Australia, †CEBAF and Old Dominion University

\section{b. Variational and Green's Function Monte Carlo Calculations of Few-Body Nuclear Ground States (R. B. Wiringa, A. Arriaga,* J. Carlson, $\uparrow$ V. R. Pandharipande, $\ddagger$ and B. Pudliner $\ddagger$ )}

We continue to develop our variational Monte Carlo (VMC) calculations for the ground states of ${ }^{3} \mathrm{H},{ }^{3} \mathrm{He},{ }^{4} \mathrm{He},{ }^{5} \mathrm{He},{ }^{6} \mathrm{He}$ and ${ }^{6} \mathrm{Li}$ using nuclear Hamiltonians with realistic two- and three-nucleon potentials. The variational wave functions include central, spin, isospin, tensor, and spin-orbit two-body correlations and three-body correlations for the three-nucleon potential. In the last year we have also added extra three-body correlations for the two-body potential. These trial fuıctions now give upper bounds to the ground-state binding energy $\sim 2 \%$ above Faddeev calculations in ${ }^{3} \mathrm{H}$ and Green's Function Monte Carlo (GFMC) calculations in ${ }^{4} \mathrm{He}$. We are now computing with the new Argonne $v_{18}$ model (Sec. I.B.a.) and the Urbana IX three-nucleon potential, which is chosen to reproduce the three- and four-body binding energy in exact calculations. One feature of the $\mathrm{v}_{18}$ calculations is an improved value for the ${ }^{3} \mathrm{H}-{ }^{3} \mathrm{He}$ mass difference due to the charge asymmetry included in the NN potential.

The current five- and six-body nuclei calculations are less satisfactory. The ${ }^{5} \mathrm{He}(\alpha-n$ scattering) variational energies have been $\sim 6 \%$ above the available GFMC results. The ${ }^{6} \mathrm{Li}$ variational calculation currently gives about $0.6 \mathrm{MeV}$ more binding energy than the $\alpha$ with the Argonne $\mathrm{v}_{18}$ + Urbana IX interaction, but this is less than a separated $\alpha$-d pair, so the solution is not stable against breakup.

The VMC code was optimized to run on the Nationai Energy Research Supercomputer Center (NERSC) Cray YMP-C90 and achieved a speed of $\sim 360$ MFLOPS on a single C90 processor. The code has now been adapted for the new 128-processor IBM SP1 parallel supercomputer in Argonne's Mathematics and Computer Science Division, and we have achieved speeds of $\sim 6$ GFLOPS. This improved performance makes a GFMC calculation for ${ }^{6} \mathrm{Li}$ seem feasible, and we are in the process of preparing such a program. The six-body nuclei (like ${ }^{16} \mathrm{O}$ and ${ }^{40} \mathrm{Ca}$ discussed in Sec. B.c.) may provide a sensitive test of components of our model interactions, particularly the p-wave parts of the NN potential, that are not tested in the s-shell nuclei.

*University of Lisbon, Portugal, $\nmid$ Los Alamos National Laboratory, $\ddagger$ University of Illinois 


\section{c. Ground States of Larger Nuclei (S. C. Pieper, R. B. Wiringa, and V. R. Pandharipande*)}

The methods (Sec. B.b.) used for the few-body nuclei require operations on the complete spinisospin vector; the size of this vector makes such methods : mpractical for nuclei with $A>8$. During the last few years we developed cluster expansion methods that do not require operations on the complete vector. We use the same Hamiltonians for the few-body nuclei and variational wave functions whose form is similar to the few-body wave functions. The cluster expansions are made for the noncentral parts of the wave functions and for the operators whose expectation values are being evaluated. The central pair correlations in the wave functions are treated exactly and this requires the evaluation of 3A-dimensional integrals which are done with Monte Carlo techniques. Most of our effort has been on ${ }^{16} \mathrm{O}$, other $\mathrm{p}$-shell nuclei, and ${ }^{40} \mathrm{Ca}$.

This year Argonne's Mathematics and Computer Science Division acquired a 128-processor IBM SP1 which has a theoretical peak speed of 16 GFLOPS. We converted our program to run on this machine. Because of the large memory on each node of the SPI, it was easy to convert the program to parallel form with very low communicationoverhead. Considerably more effort was needed to restructure the program from one oriented towards long vectors for the $C_{1}$ ay computers at NERSC to one that makes efficient use of the cache of the RS6000 architecture. With these changes, the program runs at 48 MFLOPS on a single processor or more than 6 GFLOPS on the whole machine, about 13 times faster than the speed on a single processor of the Cray YMP-C90 at NERSC.

The SPI has made extensive calculations of ${ }^{40} \mathrm{Ca}$ possible for the first time. We are doing these with the new Argonne $v_{18}$ (Sec. B.a.) two-nucleon potential and Urbana IX three-nucleon potential. We can obtain a statistical error of $0.2 \mathrm{MeV} /$ nucleon in a one-day run, however the cluster expansion for the three-nucleon potential energy is considerably slower than for ${ }^{16} \mathrm{O}$. We are presently attempting to devise a revised expansion, making use of nuclear-matter summation techniques.

We also used the SPI to make extensive variational searches, using the new two- and three-body potentials, for the ${ }^{16} \mathrm{O}$ wave function. Because of the high speed of the SPI, we can do the variational search with Monte Carlo walks some 10 to 20 times longer ( 3 to 4 times smaller variance) than used to be possible. We have found that $v_{18}$ has two significant improvements over $v_{14}$ : 1) $v_{18}$ results in 160 being $0.7 \mathrm{MeV} /$ nucleon more bound than ${ }^{4} \mathrm{He}$. With $\mathrm{v}_{14}$ we obtained only $0.2 \mathrm{MeV}$ more binding; the experimental value is $0.9 \mathrm{MeV}$. At least part of this enhanced relative binding appears to be due to the stronger $p$-wave aitraction in $v_{18}$; we intend to further study this effect. 2) The density of $16 \mathrm{O}$ obtained with $v_{14}$ had a very deep minimum at the origin and a large overshoot at a radius of $1.5 \mathrm{fm}$. This resulted in a poor prediction of the charge form factor. The density with $v_{18}$ is much closer to the experimental value.

*University of Illinois

d. Nucleon, Nucleon-Cluster, $\Delta$-Isobar and Excess Pion Momentum Distributions in Finite Nuclei (R. B. Wiringa, S. C. Pieper, J. Carlson,* V. R. Pandharipande, $†$ and R. Schiavilla $\ddagger$

We computed a variety of momentum distributions in ${ }^{2} \mathrm{H},{ }^{3} \mathrm{He},{ }^{4} \mathrm{He}$, and ${ }^{16} \mathrm{O}$. These include proton and neutron distributions in these four nuclei, deuterons in ${ }^{3} \mathrm{He}$ and ${ }^{4} \mathrm{He}$, and tritons in ${ }^{4} \mathrm{He}$. The calculations are based on correlated variational wave functions for the Argonne $v_{14}$ two-nucleon and Urbana three-nucleon interactions. The nucleon momentum distributions use an improved Monte Carlo sampling procedure, where the initial and final positions of the

\footnotetext{
*Los Alamos National Laboratory, †University of Illinois, $\ddagger$ CEBAF and Old Dominion University
} 
sampled nucleon are shifted by equal but opposite amounts. The result is a much smaller variance than previously achieved which allows calculations up to $\sim 8 \mathrm{fm}^{-1}$. The deuteron and triton momentum distributio:s also go to much higher momenta than obtained previously.

We also calculated $\Delta$-isobar momentum distributions in ${ }^{2} \mathrm{H},{ }^{3} \mathrm{He}$, and ${ }^{4} \mathrm{He}$, using the Argonne $v_{18}$ potential to build explicit $\Delta$ components into our variational wave functions. These give a total $\Delta$-isobar content of $0.5 \%$ in ${ }^{2} \mathrm{H}$ and $3.1 \%$ in ${ }^{4} \mathrm{He}$. The same correlations were used to obtain improved excess-pion momentum distributions in these nuclei and in $16 \mathrm{O}$. Relatively little work was done on this problem this year, as we worked on updating the Hamiltonian. However we expect to repeat the calculations with the new interaction of Sec. B.a. and to add ${ }^{6} \mathrm{Li}$ and ${ }^{40} \mathrm{Ca}$ to the list of nuclei to be studied.

e. Coulomb Sum and Proton-Proton Correlations in Nuclei (R. B. Wiringa, S. C. Pieper, J. Carlson, ${ }^{*}$ and R. Schiavilla $\dagger$ )

For simple models of the nuclear charge operator, measurements of the Coulomb sum and charge form factor of a nucleus directly determine the proton-proton correiations. Experimental results for ${ }^{3} \mathrm{H}$ and ${ }^{3} \mathrm{He}$ obtained at Bates and Saclay were found to be in disagreement with theoretical calculations based on realistic wave functions and a one-body charge operator. The derived proton-proton correlation $\rho_{\mathrm{pp}}(\mathrm{k})$ was found to have a zero at lower momentum transfer, and much greater strength in the region of the second maximum. This was interpreted as evidence for a smaller proton-proton distribution function at short distances and a stronger repulsion than current theoretical models provide. A similar discrepancy was observed earlier for the elastic electromagnetic form factors. There the theoretical calculations were brought into agreement with experiment by constructing better charge and current operators, including one-body relativistic and two-body meson-exchange contributions.

We have examined current experimental results for light nuclei from Bates and Saclay with the same charge operator used to explain successfully the charge form factor. It includes DarwinFoldy, neutron, and spin-orbit one-body terms and $\pi, \rho$, and $\omega$ exchanges and other two-body terms. Because of these additional terms, it is more appropriate to talk about extracting a longitudinal-longitudinal correlation $\rho_{L L}(k)$. To obtain $\rho_{L L}$ from experiment it is necessary to measure both the charge form factor $F_{L}$ and Coulomb sum $S_{L}$. An important step in forming the Coulomb sum is to estimate the unobserved strength in the longitudinal response beyond the maximum energy loss that is measured. This was done by requiring the experimental and theoretical energy-weighted sum rules to match.

We find that the extracted and theoretical curves for $\rho_{L L}(k)$ in ${ }^{3} \mathrm{He}$ and ${ }^{4} \mathrm{He}$ are in excellent agreement, but there is some discrepancy in ${ }^{3} \mathrm{H}$. We conclude that due to the complicated nature of the coupling between a longitudinal photon and the nucleus, even at low momentum transfers, the $\rho_{L L}$ extracted from (e,e') inclusive data cannot provide direct information on the strength of the proton-proton repulsive interaction at short range. A paper on the few-body calculations was published in Phys. Rev. Lett. 25, 3856 (1993). We plan to extend the calculations to $16 \mathrm{O}$ and ${ }^{40} \mathrm{Ca}$ in the future.

* Los Alamos National Laboratory, fOld Dominion University and CEBAF 


\section{f. Monte Carlo Calculations of (e,e'p) Reactions (S. C. Pieper, V. R. Pandharipande*, S. Boffi, $\dagger$ and M. Radici $†$ )}

A collaboration was initiated with the University of Pavia to carry out detailed calculations of ${ }^{16} \mathrm{O}\left(\mathrm{e}, \mathrm{e}^{\prime} \mathrm{p}\right)$ reactions for which there is precise NIKHEF data. We will use final-state wave functions containing a product of the distorted wave, the variational ${ }^{15} \mathrm{~N}$ wave function, and noncentral correlations between the ejected proton and the nucleons in $15 \mathrm{~N}$. The Pavia group previded a subroutine that generates the distorted wave which is suitable for use in the Argonne nuclear Monte Carlo program. Last year we incorporated ${ }^{\prime} .0$ subroutine into the program and wrote the routines to add the $\mathrm{NN}$ and $\mathrm{NNN}$ correlations and to evaluate the expectation values in the impulse approximation. Preliminary calculations showed unexpectedly small effects from the correlations.

In an attempt to understand this result we computed the $\mathrm{p}_{3 / 2}$ quasihole wave function in 160 and used it in the Pavia (e,e'p) program. We also developed a local-density approximation (LDA) for obtaining the quasihole wave function from a mean-field wave function, and studied the effects of using this LDA on the outgoing distorted waves. We find that we can predict correctly the contribution of the interior of the nucleus to the observed (e,e'p) cross sections, but the surface contribution is too large.

*University of Illinois, $\nmid$ Uni ${ }_{\text {. }}$ jity of Pavia, Italy

\section{g. Isovector Spin-Longitudinal and -Transverse Response of Nuclei (S. C. Pieper,} R. B. Wiringa, V. R. Pandharipande,* J. Carlson, $\nmid$ and R. Schiavilla $\ddagger$ )

Recently polarized $(\mathrm{p}, \mathrm{n})$ experiments were made at Los Alamos to measure the ratio of isovector spin-longitudinal (IVSL) and -transverse (IVST) responses of ${ }^{12} \mathrm{C}$ and ${ }^{40} \mathrm{Ca}$. These were compared to calculations using the random-phase approximation (RPA) and distorted-wave impulse approximation. The calculations predict an enhancement of the IVSL response over the IVST at low energy transfers, but this enhancement was not observed.

It is not yet possible to compute response functions for nuclei larger than ${ }^{4} \mathrm{He}$ using the wave functions of Sec. B.c., but integrals over the energy transfer (sums) can be easily evaluated. We computed the sums and energy-weighted sums of the IVSL and IVST for ${ }^{4} \mathrm{He},{ }^{16} \mathrm{O}$ and nuclear matter. We also computed the Laplace transform of the responses (Euclidean response) for ${ }^{2} \mathrm{H}$ and ${ }^{4} \mathrm{He}$ and of the RPA responses for ${ }^{12} \mathrm{C}$. Comparing these calculations shows that the strong tensor-isospin correlations in our realistic nuclear wave functions push the resporse to higher energies than is indicated by RPA calculations, which do not include such correlations (see Fig. IV-4).

Our results point out the need for a more sophisticated theory than RPA to make comparison with the IVSL/IVST experiments. However, because our calculations were made with only plane waves, they cannot be compared directly to experiment; we are considering extending our calculations to include distorted waves. We point out that reliable calculations of the responses in ${ }^{2} \mathrm{H}$ and ${ }^{4} \mathrm{He}$ should be possible and urge experimentalists to consider such experiments. A first paper on this work was published in Phys. Rev. C 49, 789 (1994).

*University of Illinois, $†$ Los Alamos National Laboratory, $\ddagger$ Old Dominion University and CEBAF 


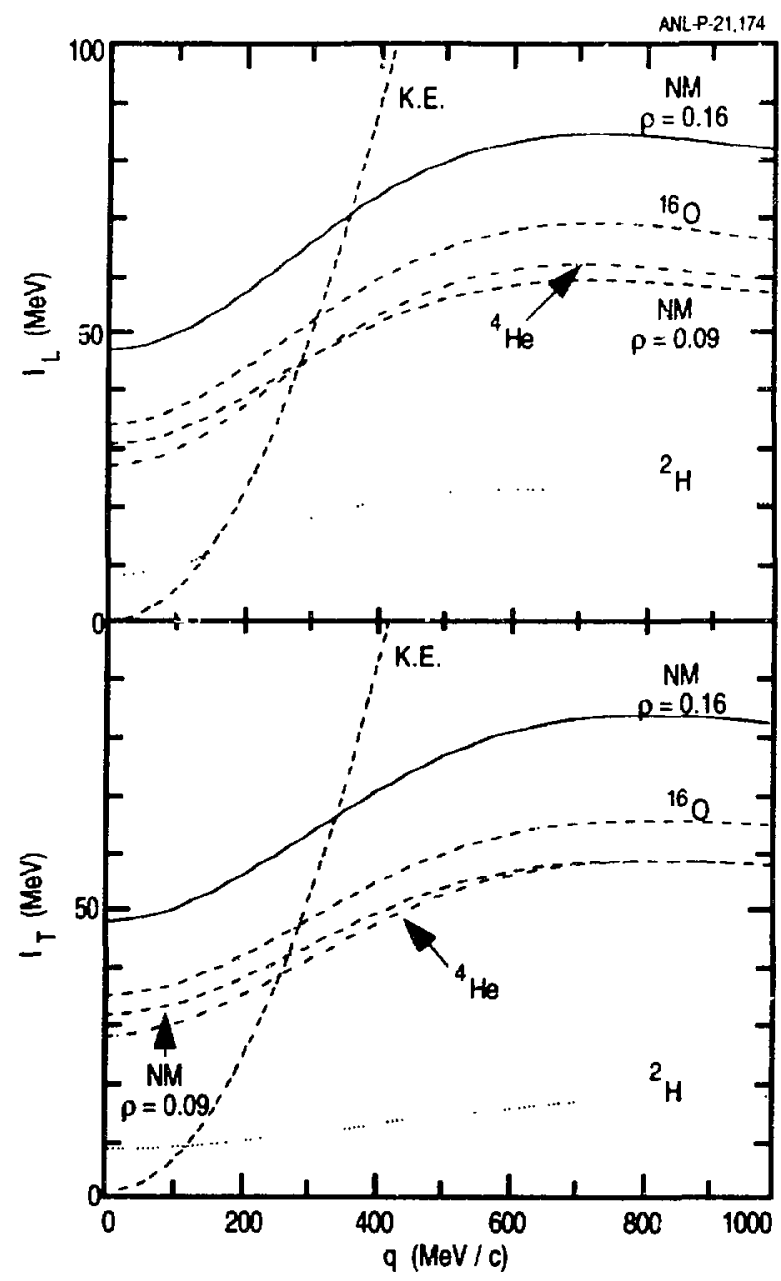

Figure IV-4. The interaction contributions $I_{L}$ and $I_{T}$ to the energy-weighted sums of IVSL and IVST responses of ${ }^{2} \mathrm{H},{ }^{4} \mathrm{He},{ }^{15} \mathrm{O}$ and $\mathrm{NM}$ at $\rho=0.09$ and $0.16 \mathrm{fm}^{-3}$. The curve labeled K.E. shows the kinetic energy contribution, the only contribution used in the RPA.

h. Nuclear and Neutron Matter Studies (R. B. Wiringa, A. Fabrocini,* and V. R. Pandharipande†)

Nuclear and neutron matter remain an area of continuing interest. A major study of the dense nucleon matter equation of state, neutron star properties, and nucleon optical potential, for realistic Hamiltonians including three-nucleon potentials, was completed in 1988. This work, coupled with our studies of finite nuclei, showed that the addition of plausible three-nucleon potentials can make a significart improvement in calculated binding energies and saturation properties. Subsequent calculations in finite nuclei benefited significantly from the introduction of three-body correlations for the three-body potential. We are now investigating ways to introduce such correlations in our matter calculations. The ${ }^{16} \mathrm{O}$ calculations (Sec. I.B.c) also pointed out a possible discrepancy in the evaluation of L-dependent potential contributions in matter that we are now reviewing. During the current year we worked on adding four-body elementary diagrams to the Fermi hypernetted chain integral equations (FHNC/4) that are used to evaluate expectation values in matter. The corrections are expected to be small at normal

* University of Pisa, Italy, $\dagger$ University of Illinois 
density, but may be significant in high-density matter, and may also correct some problems in matter sum rules. We are now evaluating the energy with our new Hamiltonian (Sec B.a) in both nuclear and neutron matter. We also made a first calculation of the superfluid energy gap in neutron matter, which is relevant to neutron star cooling and timing glitches. We plan to pursue these studies in the coming year.

\section{i. Ground State of Hypernuclei (S. C. Pieper, A. Usmani *, and Q. N. Usmani*)}

The variational Monte Carlo calculation of nuclei (Secs. B.b. and B.c.) was adapted for hypernuclei such as ${ }_{4}^{17} \mathrm{O},{ }_{4}^{16} \mathrm{O},{ }_{\Lambda}^{12} \mathrm{C}$. In this calculation we use the same realistic nuclear Hamiltonians that we use for normal nuclei with the addition of phenomenological $N \Lambda$ and NN $\Lambda$ potentials such as those studied previously by Bodmer and Usmani. The wave function is also of the same form as in rormal nuclei with additional $\mathbf{N} \Lambda$ and $\mathbf{N} N \Lambda$ non-central correlations. The deveiopment work for these calculations was done principally by A. Usmani and $Q$. N. Usmani at Jamia Millia. Final production calculations are being done on the NERSC computers.

During the last fiscal year, the necessary modifications were completed and debugged. We found that the $\Lambda \mathrm{NN}$ correlations induced by the $\Lambda \mathrm{NN}$ potential are much more important than the corresponding NNN correlations; in fact, they change the sign of the contribution of the $\Lambda \mathrm{NN}$ potential. Calculations of ${ }^{17} \mathrm{O}$ have been completed; when corresponding calculations of ${ }_{\Lambda}^{5} \mathrm{He}$ are completed the work will be written up for publication.

*Jamia Millia Islamia, New Delhi, India

j. $\quad \Lambda$ Single-Particle Energies (A. R. Bodmer, Q. N. Usmani,* and M. Sami*)

We have essentially completed our work on the $\Lambda$ hyperon single-particle (s.p.) energies and their interpretation in terms of the basic $\Lambda$-nuclear interactions. Significant new results in FY 1994 were: a) We obtained about $30 \mathrm{MeV}$ repulsive contribution of the three-body $\Lambda \mathrm{NN}$ forces in nuclear matter. b) We were able to exclude purely "dispersive" $\Lambda N N$ forces. c) We were able to obtain satisfactory fits to the s.p. data with a combination of dispersive and two-pion-exchange $\Lambda N N$ forces. For such interactions, which provide a fit to the s.p. data, the $\Lambda$ binding energy as a function of the nuclear matter density shows characteristic saturation features with a maximum at a density not very different from that of normal nuclear matter. d) We obtained a more precise measure of the space-exchange part of the $\Lambda$-nuclear force than was previously available, corresponding to an exchange parameter $\simeq 0.32$. The space-exchange force is rather directly related to the effective mass of a $\Lambda$ in the nuclear medium and turns out to be about $70 \%$ of its free mass. As a result, we also obtain a much better value for the p-state $\Lambda$-nucleus potential which is about $40 \%$ of the s-state potential. The $\Lambda$ binding to nuclear matter is determined to be $\simeq 28 \pm 1 \mathrm{MeV}$.

\section{k. Core-Nucleus Distortion in Hypernuclei (A. R. Bodmer and Q. N. Usmani*)}

We have essentially completed a study of the effects of the spherical distortion of the "core" nucleus by the $\Lambda$ in a hypernucleus. The response of the core was determined by an appropriately chosen energy-density functional which depends, in particular, on the nuclear compressibility. The forcing action of the $\Lambda$ is determined by the nuclear density dependence of the $\Lambda$ binding in nuclear matter which is obtained from our work on the $\Lambda$ single-particle energies. Because of the strongly repulsive $\Lambda N N$ forces, this $\Lambda$ binding "saturates" at a density

\footnotetext{
*Jamia Millia Islamia, New Delhi, India
} 
close to the central density of nuclei, and results in a reduced core-nucleus distortion much less than would otherwise be obtained. The effects of the core distortion then turn out to be very small even for quite light hypernuclei. This result justifies the assumption that spherical core nuclei are effectively undistorted in a hypernucleus.

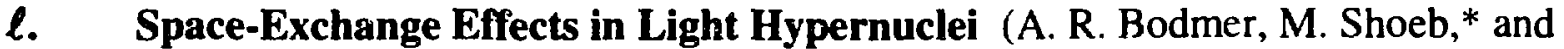 Q. N. Usmani†)}

We made variational Monte Carlo calculations of the ground-state $\Lambda$ separation energies $B_{\Lambda}$ of the s-shell hypernuclei $\left({ }_{\Lambda}^{3} \mathrm{H},{ }_{\Lambda}^{4} \mathrm{H}, 5 \mathrm{He}\right)$ and of ${ }_{\Lambda}^{9} \mathrm{Be}$ for interactions which include central $\Lambda \mathrm{N}$ potentials with a space-exchange component and three-body $\Lambda N N$ forces. The s-shell hypernuclei are treated as $A$-body systems $(A=$ baryon number $)$, and ${ }_{A}^{9} \mathrm{Be}$ is analyzed in a $\Lambda+2 \alpha$ model. The reduction of $B_{\Lambda}$ due to a space-exchange $\Lambda N$ potential was obtained for the s-shell hypernuclei. For ${ }_{\Lambda}^{3} \mathrm{H},{ }_{\Lambda}^{4} \mathrm{H}$ and ${ }_{\Lambda}^{5} \mathrm{He}$, the exchange energy is approximately $0.04,0.15$ and $0.5 \mathrm{MeV}$ respectively. For ${ }_{\Lambda}^{9} \mathrm{Be}$ a more limited study gives about $1.3 \mathrm{MeV}$. These values are much larger than for soft potentials for which the correlations are weak.

*Muslim University, Aligarh, India, †Jamia Millia Islamia, New Delhi, India

m. Charge-Symmetry Breaking $\Lambda$-Nucleon Interaction (A. R. Bodmer, M. Murali,* and Q. N. Usmani*)

Some time ago we showed that the charge symmetry breaking interaction as obtained from the mass four hypernuclei $\left({ }_{\Lambda}^{4} \mathrm{H},{ }_{\Lambda}^{4} \mathrm{He}\right)$ was spin independent; a result which cannot be understood with the conventional meson-exchange models. The calculations of $\left({ }_{4}^{4} \mathrm{H}, 4 \mathrm{He}\right)$ are currently being extended to include noncentral nuclear and hypernuclear forces which could modify this result. At a more fundamental level we intend to study quark-structure contributions to the charge-symmetry breaking interaction.

\section{n. Suppression of the $\Lambda-\Sigma$ Coupling in Nuclear Matter (A. R. Bodmer and Q. N. Usmani*)}

We initiated a study of the modification of the coupling of the $\Lambda \mathrm{N}$ to the $\Sigma \mathrm{N}$ channel in nuclear matter with the Fermi hypernetted-chain variational approach. This modification of the $\Lambda N-\Sigma N$ coupling is a central problem in hypernuclear physics and is related closely to the strongly repulsive three-body forces which are needed to account for hypernuclear binding energies. All earlier calculations have only considered this problem in the so-called G-matrix approximation which neglects important higher-order effects. An important result of this work will be a better understanding of the density dependence of $\Lambda$ binding in nuclear matter, which can then be tested in the calculation of the $\Lambda$ single-particle energies.

*Jamia Millia Islamia, New Delhi, India

\section{HEAVY-ION REACTIONS}

The structure and reactions of nuclei far from stability have become an integral part of this research program. This new direction is related closr ly to ongoing experimental heavy-ion programs and future radioactive beam facilities. We developed a three-body model for neutron rich nuclei and used it to investigate fragmentation reactions involving the nucleus ${ }^{11} \mathrm{Li}$. The model provides a good description of most of the reaction data that were obtained. We also developed a neutron-core Hamiltonian model to describe the positive-parity states of one-neutron halo nuclei and applied it to ${ }^{11} \mathrm{Be}$. 
The studies of heavy-ion reactions at energies close to the Coulomb barrier are based on applications of the coupled-channels technique. The objective is to obtain a consistent, unified, quantum-mechanical explanation of fusion, elastic and inelastic scattering, transfer reactions and compound nucleus spin distributions. The calculations are constrained by the nuclear structure properties of the two interacting nuclei.

\section{a. Fragmentation Reactions of ${ }^{11} \mathbf{L i}$ (H. Esbensen and G. F. Bertsch*)}

We applied our three-body model for ${ }^{11} \mathrm{Li}$ (described as a ${ }^{9} \mathrm{Li}$ core and two interacting, looselybound valence neutrons) to calculate interaction and two-neutron removal cross sections. The nuclear part of these cross sections is calculated in an eikonal approximation, based on free nucleon-nucleon cross sections. The part that is due to Coulomb dissociation is calculated from the dipole response of ${ }^{11} \mathrm{Li}$ that we obtained previously. Our model reproduces quite accurately the measurements that were performed at $800-\mathrm{MeV}$ per nucleon. This work was published. Fragmentation cross sections for ${ }^{11} \mathrm{Li}$ were measured at several beam energies down to $28 \mathrm{MeV}$ per nucleon. We applied our model and made comparisons to the new data. The model reproduces the target and the energy dependence of the measured cross sections fairly well, but it tends to under-nredict slightly the measurements at the lowest beam energies. We are studying the significance of various corrections, in particular of higher-order dynamical effects, which are expected to become important at low beam energies.

*Institute for Nuclear Theory, University of Washington

b. Momentum Distributions for $\left({ }^{11} \mathbf{L i},{ }^{9} \mathrm{Li}+\mathrm{n}+\mathrm{n}\right)$ Three-Body Breakup Reactions (H. Esbensen, G. F. Bertsch, ${ }^{*}$ and others $\dagger$ )

Three-body coincidence measurements of the ${ }^{11} \mathrm{Li} \rightarrow{ }^{9} \mathrm{Li}+n+n$ breakup, induced by interactions with a high $\cdot \mathrm{Z}$ target, can provide detailed information about the low-lying dipole response of ${ }^{11} \mathrm{Li}$.

Such measurements have been performed at Michigan State University, at a beam energy of 28 $\mathrm{MeV}$ per nucleon on a lead target. Coulomb dissociation is by far the most dominant reaction mechanism, and the data were analyzed assuming this reaction mechanism. This work was published in Phys. Rev. C 48, 326 (1993).

We applied our three-body model for ${ }^{1}{ }^{1} \mathrm{Li}$ to calculate the momentum distributions for the threebody breakup, induced by Coulomb dipole excitations. The calculated momentum distribution for the relative motion of the two emitted neutrons is in remarkably good agieement with the measurement, and so is the single-neutron momentum distribution. The measured momentum distribution of the ${ }^{9} \mathrm{Li}$ fragment, on the other hand, is shifted to higher momenta compared to the prediction. The measured decay energy spectrum and the extracted dipole strength distribution are also shifted towards higher excitations comp?red to our predictions. This work has been published.

The measurements show that the average velocity of the ${ }^{9} \mathrm{Li}$ fragments is larger than that of the emitted neutrons. We are investigating a possible explanation that this shift is due to a higherorder polarization effect, which was used to explain the difference between the stopping powers of protons and anti-protons in solids. This mechanism could possibly also be the reason for the discrepancy between the calculated and the extracted dipole strength distribution of ${ }^{11} \mathrm{Li}$. If the polarization effect really plays an important role it would be easy to reduce its impact in future measurements, since it decreases as one over the third power of the beam velocity.

*Institute of Nuclear Theory, University of Washington, $\uparrow$ Michigan State University collaboration 


\section{c. Momentum Distributions for Nuclear Induced Breakup Reactions \\ (H. Esbensen, M. Pirovano*, R. A. Broglia*, E. Vigezzi,* and F. Barranco $\left.{ }^{\dagger}\right)$}

We used our three-body model for ${ }^{11} \mathrm{Li}$ to calculate momentum distributions for the ${ }^{11} \mathrm{Li} \rightarrow{ }^{9} \mathrm{Li}+\mathrm{n}+\mathrm{n}$ three-body breakup, induced by the nuclear field from a light target. Cross sections and momentum distributions were obtained from the nuclear multipole response of the valence neutrons in ${ }^{11} \mathrm{Li}$, calculated at different impact parameters from the target. Our calculations include the effect of the final-state interaction between the two valence neutrons. The calculated momentum distributions are broader than those obtained for Coulomb-induced reactions. They can be tested in future three-body coincidence measurements on a light target.

*Istituto Nazionale di Fisica Nucleare, Milano, Italy, †University of Sevilla, Spain

\section{d. Positive-Parity States in ${ }^{11 B e}$ and ${ }^{13} \mathrm{C}$ (H. Esbensen, H. Sagawa*, B. A. Brown, $\dagger$ and others $\ddagger$ )}

Our previous study of the parity inversion in ${ }^{11} \mathrm{Be}$ suggested that the positive-parity spectri in may be described in terms of a simple neutron-core Hamiltonian, which includes a nucl::ar quadrupole coupling between the valence neutron and a deformed core. We developed such a model Hamiltonian, with a quadrupole coupling strength that is consistent with measured B(E2) values. The model describes the low-lying positive-parity states in ${ }^{11} \mathrm{Be}$ and ${ }^{13} \mathrm{C}$ rather well. The ${ }^{13} \mathrm{C}$ spectrum is consistent with an oblate deformation of the core, whereas the ${ }^{11} \mathrm{Be}$ spectrum favors a prolate deformation. The $1 / 2^{+}$ground-state wave function that we obtain for ${ }^{11} \mathrm{Be}$ consists mainly of an $\mathrm{s}_{1 / 2}$ single-particle state coupled to the $0^{+}$ground state of the core $(86 \%)$, and a $\mathrm{d}_{5 / 2}$ state coupled to the excited $2^{+}$core state $(11 \%)$. This decomposition is in reasonable agreement with the measured spectroscopic factor and shell-model calculations. This work was published in Phys. Lett. B309, 1 (1993).

The simplicity of the model makes it easy to apply it in reaction calculations. An example is the angular distribution of neutrons emitted in ( $\left.1{ }^{1} \mathrm{Be},{ }^{10} \mathrm{Be}\right)$ breakup reactions, which was measured recently at GANIL. This application was published in Phys. Lett. B304, 55, (1993).

\footnotetext{
*University of Tokyo, Japan, †Michigan State University, $¥$ GANIL collaboration
}

\section{e. Heavy-ion Reactions near the Coulomb Barrier (H. Esbensen)}

Coupled-channels calculations of heavy-ion reactions become increasingly difficult for heavy systems, due to strong couplings and the importance of multi-step processes of inelastic excitations and nucleon transfers. Detailed measurements of reactions between different $\mathrm{Ni}$ anỏ Mo isotopes, and recently also between $\mathrm{Ni}$ and $\mathrm{Kr}$ isotopes, were performed at ATLAS and revealed a correlation between large multi-nucleon transfer cross sections and an enhancement of sub-barrier fusion cross sections. The goal of this study is to achieve a consistent coupledchannels description of heavy-ion reactions near the Coulomb barrier and, in particular, to develop a realistic model for multi-step processes. These studies are performed in collaboration with experimentalists and has resulted in a joint publication [Phys. Lett. B317, 31 (1993)] on fusion reactions between $\mathrm{Ni}$ and Mo isotopes. 


\section{f. Spin Distributions in Heavy-Ion Fusion Reactions (S. Landowne)}

The traditional barrier penetration model for fusion reactions, as well as its modern extension to include effects of direct reaction channel couplings, predicts a close correlation between the energy dependence of the fusion reaction cross section and the distribution of angular momentum forming the compound nucleus. The spin distribution is required for the subsequent statistical analysis of the compound nuclear decay. Data analyses have suggested a number of cases of heavy-ion reactions where the predicted correlation appears to be violated. If these analyses hold up, they point toward a new, unknown mechanism affecting the fusion rates of heavy nuclei. One of the best theoretical studies of heavy-ion fusion reactions was done for the well-known series of $\mathrm{Ni}+\mathrm{Ni}$ collisions. Our coupled-channel calculations reproduce simultaneously the available $\mathrm{Ni}+\mathrm{Ni}$ data for fusion, elastic scattering, and direct reaction cross sections. The corresponding theoretical spin distributions are now compared to new measurements carried out at Legnaro. The agreement is remarkably good.

\section{NUCLEAR STRUCTURE STUDIES}

The goal of this program is to understand the correlations in nuclear states arising from effective two-body interactions. In the past few years, the emphasis of this program was on superdeformation at low and high spins. In strongly deformed nuclides, the correlation effects are manifest and can be treated in such cases through the introduction of a deformed singleparticle potential. Similarly, when pairing forces are strong, a quasi-particle approximation is adequate for treating them. However, when a deformation mode is weak, or when pairing correlations are small, a one-body treatment is not adequate for obtaining a reasonable description of nuclear states. It is a major challenge of nuclear structure theory to devise methods of treating such problems. In our studies, we utilize both the deformed single-particle potential methods and many-body wave functions to understand nuclear properties. In the context of the Strutinsky method, we studied the effects of angular momentum in inducing changes in nuclear shapes in many different regions of the periodic table. In the past year, we were interested in studying very extended nuclear shapes (axis ratios $>2: 1$ ). We found that conventional treatments in terms of quadrupole and hexadecapole deformations are not adequate for treating these very extended shapes. We found that the introduction of a necking degree of freedom into the parameterization of the nuclear shape gives a markedly improved description. We are also studying these very extended shapes from a different perspective: cranked HartreeFock-Bogoliubov calculations, using the Gogny force. Using both methods to study ${ }^{182} \mathrm{Os}$, we find a superdeformed and very extended nuclear shape. Both methods give almost exactly the same axis ratios in the two minima. An experimental search was undertaken at Argonne to identify states in both the super-deformed and very extended minima of ${ }^{182} \mathrm{Os}$.

One of the major results of our research program was the prediction of a new, accessible region of superdeformed nuclides around ${ }^{192} \mathrm{Hg}$. Superdeformation was subsequently found in this region, first by the experimental group at Argonne. The study of this region by the experimental groups at Argonne, Daresbury and Berkeley-Livermore has since led to the discovery of superdeformation in many nuclides. A great surprise in the study of superdeformation was the observation of identical transition energies in rotational bands of different nuclei. Following the observation of identical bands in the A - 150 region, many such pairs of bands were found in the A $\sim 190$ mass region. It has since been pointed out that such bands are also found in normally deformed nuclei. In order to get some understanding of the adequacy of conventional methods of calculating rotational properties, we went beyond mean-field approximations. Making use of many-body wave functions, we studied the superdeformed band in ${ }^{192} \mathrm{Hg}$ and got substantial corrections to the conventional treatments. 
Another subject of interest is the nuclear structure of the heaviest elements, and the utilization of heavy element nuclear structure information to determine the limits if stability of superheavy elements.

All of these problems require large amounts of supercomputer time. The use of massivelyparallel supercomputers offers the promise of substantial progress in our understanding of nuclear structure. We plan to modify our codes to exploit these computers insofar as it is possible. Our research program includes a strong collaboration with experimental programs at Argonne.

\section{a. Configuration Interaction Effects in Rotational Bands of Superdeformed Nuclei} (R. R. Chasman)

An unexpected result, that was found in the experimental investigation of superdeformed rotational bands, is the observation of near identical dynamic moments of inertia in different nuclei. This phenomenon was also noted in normally deformed rotational bands. This motivated us to undertake a calculation of moments of inertia going beyond the usual BCS treatment of pairing correlations. Before one does calculations that purport to show that transition energies are identical in two different rotational bands, it is important to understand the adequacy of calculations of rotational spacings in a single rotational band. To this end, we extended the conventional treatment of rotational spacings. We developed a treatment of rotation using many-body wave functions with good particle-number in order to investigate the validity of conventional treatments of the properties of rotational bands. Our many-body wave functions are products of sums of terms and each such state has $\sim 10^{12}$ amplitudes, and is constructed with $\sim 2000$ variational parameters. We minimize the variational parameters after projecting out states of good particle number, good parity and good signature. Our goal, at this time, is to learn how to reliably calculate energies in a single rotational band. Only when we can do this, does it become reasonable to try to calculate the small differences in energy $(\sim 1$ $\mathrm{keV}$ ) that are characteristic of identical bands.

A priori, the BCS method is suspect for total angular momentum I = 0 for the treatment of superdeformed nuclear shapes because the single-particle level density near the nuclear surface is small. If it were large, there would be no superdeformed minimum. At high spin pairing correlations are further weakened, and the BCS method becomes even worse. Our calculations go beyond the conventional calculations in two ways: (1) we use wave functions with good particle number and; (2) we do a configuration interaction calculaticis. We use the proton and neutron pairing interaction strengths to generate configurations in the configuration interaction calculations. We also include states obtained at different cranking frequencies. This approach was used to study the superdeformed rotational band in ${ }^{192} \mathrm{Hg}$.

The result of our calculation is that it is extremely important to take configuration interaction effects into account. We find that, above $\mathrm{I}=30 \hbar$, the energy can be lowered by $>300 \mathrm{keV}$ by including the states generated by changing the pairing interaction strengths. We get additional lowering of $\sim 200 \mathrm{keV}$ when states generated from all cranking frequencies are also included. These shifts are relative to a state with good particle number. The shifts are even larger relative tc states calculated in the BCS approximation. $T:-$ se shifts in the energies of the states give shifts of as much as $50 \mathrm{keV}$ in the energies of the $\gamma$ rays between states. In Fig. IV -5 , we display the differences between calculated and measured transition energies in the superdeformed band of $192 \mathrm{Hg}$. The inclusion of configuration interaction effects reduces the disagreement considerably. The effects become particularly large at spins that are slightly lower than the spin at which there is a sharp decrease in pairing. One effect of the configuration interaction is to spread out this decrease in pairing over many values of spin. This spreading is in addition to the change from pairing collapse to a continuous decrease in pairing that one gets by using states of good particle number rather than a BCS approximation. 
The conclusion to be drawn from these calculations is that one must go far beyond the quasiparticle approximation to deal intelligently with the issue of identical bands. This study was published in Phys. Lett. B319, 41 (1993).

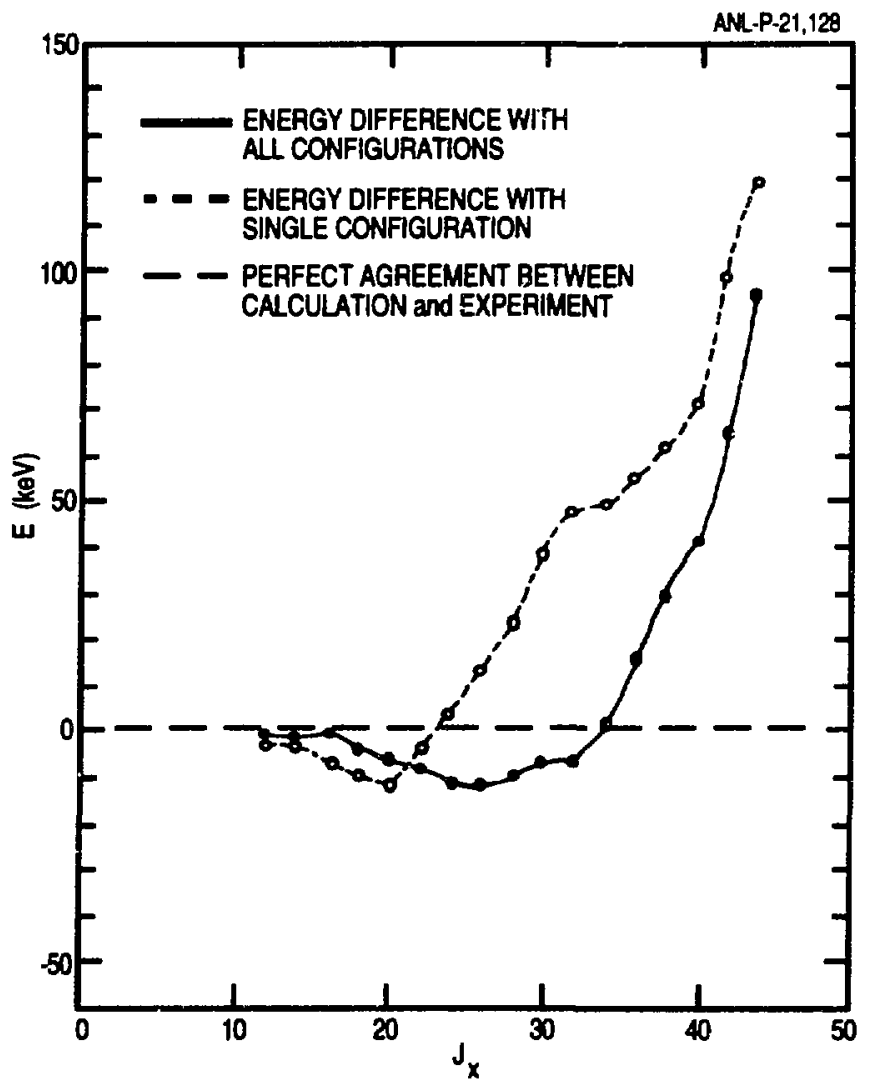

Fig. IV-5. Comparison of experimental and theoretical transition energies in superdeformed band of ${ }^{192} \mathrm{Hg}$.

\section{b. Very Extended Nuclear Shapes Near A = 180 (R. R. Chasman)}

In the past few y yars, superdeformed shapes were found at high spins in the $A=150$ and the $A=190$ mass regions. In the $A=150$ mass region, the superdeformed shapes are characterized by an axis ratio of 1.9:1. Our calculations predicted that superdeformed states would be accessible in the $A=190$ region; these were first found in the ATLAS experimental program. These nuclides have axis ratios of $\sim 1.6: 1$.

An interesting open question is whether there are other regions of nuclei having superdeformed isomers that are accessible at high spins. A particularly intriguing possibility is that there may exist accessible high-spin isomers that are even more extended than those that have been seen so far. To investigate this possibility, we carried out a series of cranked Strutinsky calculations in a three-dimensional axially-symmetric deformation space. Two of the dimensions are the usual quadrupole and hexadecapole deformations; the third is a necking-in deformation which is expected to be important for very extended shapes. With the advent of very fast vector supercomputers, it is now feasible to make investigations of a three-dimensional deformation space. In the course of these calculations, we found that the usual two-dimensional disformation space (quadrupole thexadecapole) does not provide a useful description of energy surfaces at large deformation. Our calculations show that there is a group of nuclei near $A=180$, in which isomers with very extended shapes (axis ratios of $2.2: 1$ ) might be populated in heavy-ion 
reactions. These shapes are characterized by large values of the ellipsoidal and hexadecapole deformation', with negative values of the necking-in deformation. This gives shapes that are substantially less necked in, relative to the shape given by the values of the ellipsoidal and hexadecapole deformations at the energy minimum. The best candidate nucleus is $182 \mathrm{Os}$. The effect of the necking degree of freedom is quite important for these nuclei, making the very extended minimum become yrast at I $\sim 60 \hbar$, rather than at I $\sim 70 \hbar$, as would be the case if the necking degree of freedom were not included. Our calculations were extended to very large elongations, to examine the magnitudes of the barriers of these extended minima against fission. In the best cases, we find barriers of $\sim 9 \mathrm{MeV}$ against fission at the angular momenta at which the extended shapes become yrast. Heavy-ion studies of nuclei in the $A=180$ region are being undertaken by the in-beam $\gamma$-ray spectroscopy group at Argonne.

We also find several isomers that are even more extended, characterized by axis ratios of $2.5: 1$ to as much as 3:1. In these cases, the barriers against fission are small and it seems less likely that such states can be populated in heavy-ion reactions.

The results of our first calculations of very extended shapes were published. We are including larger values of the ellipsoidal deformation in this three-dimensional deformation space that we are studying. Our new calculations in this space give a further lowering of the energy of the very extended minimum, relative to the superdeformed minimum (axis ratio $\sim 1.5: 1$ ) that also is found in this nucleus.

\section{c. High Spin Shapes in ${ }^{182}$ Os With Densîty-Dependent Forces (R. R. Chasman, J. L. Egido, ${ }^{*}$ and L. M. Robledo *)}

Both the Hartree-Fock and Strutinsky methods have advantages and disadvantages for the study of nuclear energy surfaces. In the Strutinsky method, the number of grid points goes up substantially with each additional degree of freedom that is included in the deformation space. This makes it barely feasible to use more than three dimensions in deformation space to characterize nuclear shapes. The great advantage of the Strutinsky approach is that one can study the energy surraces of many nuclei $(-300)$ with a single set of calculations. Although constrained Hartree-Fock calculations are quite time-consuming relative to Strutinsky calculations, they nave the great advantage of determining the shape at a minimum without being limited to a few deformation modes. Indeed, all modes that are not explicitly constrained in a Hartree-Fock calculation are automatically varied in such a way as to minimize the energy. We are exploiting the strengths of both methods by first carrying out Strutinsky calculations to survey a region and then carrying out Hartree-Fock calculations on the most interesting nuclei. The first nuclide we chose to study with a cranked constrained Hartree-Fock calculation is 182 Os.

Our motivations in this study are: (1) to compare the results of the two methods at high spin, in order to get some idea about the sensitivity of the results to the choice of interaction, and (2) to see whether the three deformation modes included in our Strutinsky-method study are sufficiently flexible to adequately describe nucleai shapes in the very extended minimum. One might guess that larger differences in results would be found for high spin and superdeformed shapes as compared to calculations made for low spin and for near spherical shapes. In order to examine this question in detail, we used the density-dependent Gngny interaction to study the energy surface of ${ }^{182}$ Os at $I=60 \hbar$. These shape-constrained cranked Hartree-Fock-Bogoliubov calculations were compared with our cranked Strutinsky calculations that incorporate a necking degree of freedom. In these calculations, the Gogny interaction gave two deep, well separated, very deformed minima. The first is superdeformed with an axis ratio of 1.5:1; and the second

*Universidad Autonoma de Madrio, Spain 
is very extended with an axis ratio of 2.2:1. Corrections were made in these calculations for rotational zero-point energies and for particle number fluctuations. We found that both sets of calculations give minima at the same deformations. We also found that the shapes in the minima predicted by the two methods are very similar. The very extended shape has no rounding on the sides; looking very much like a medicine capsule. The main difference between the two calculations is in the excitation energy of the two minima. At $I=60 \hbar$, the very extended minimum is excited by $\sim 2 \mathrm{MeV}$ relative to the superdeformed minimum in the cranked HartreeFock calculations. In the cranked Strutinsky calculations, the two minima are at roughly the same energy. These results were published in Phys. Lett. B322, 22 (1994). We are planning to extend these coraparisons to the study of other nuclei.

d. Search for Very Extended Shapes in ${ }^{182}$ Os (R. R. Chasman, M. P. Carpenter, R. V. F. Janssens, I. Ahmad, B. Crowell, R. G. Henry, T. L. Khoo, and T. Lauritsen)

A very important question in nuclear struciure studies is the adequacy of the Strutinsky method for states at very large deformation. According to our calculations, the nuclide 182 Os is predicted to have an experimentally accessible minimum that is more defor med than any minimum that has so far been populated at high spin in heavy-ion reactions. A study of this nuclide will provide useful tests of the Strutinsky approach and perhaps allow us to fine tune som: $\equiv$ of the ingredients of these calculations for very extendeci shapes.

An irradiation of ${ }^{138} \mathrm{Ba}$ was carried out at the LBL 88 " cyclotron with a $205-\mathrm{MeV}{ }^{48} \mathrm{Ca}$ beam in order to produce states in $182 \mathrm{Os}$ with angular momenta of $\mathrm{I} \sim 70$. The purpose of this experiment is to produce states in the very extended minimum (axis ratio 2.2:1) that were predicted in our calculations, when a necking degree of freedom is included. We also are searching for staies in the superdeformed minimum (axis ratio 1.5:1) that was predicted to be present in this nucleus. An analysis of the data taken on GAMMASPHERE is now in progress.

\section{e. Single-Particle States in the Heaviest Elements (I. Ahmad and R. R. Chasman)}

The search for superheavy elements has been a major theme of nuclear structure research for the past twenty years. Theoretical predictions of the stability of superheavy elements depend crucially on the single-particle energy level spacings in the vicinity of 114 protons and 184 neutrons. The approach that we are taiking is to learn as much as possible about these levels from spectroscopic studies of nuclides in the $A=250$ region. This is possible because there are members of the relevant spherical multiplets that drop rapidly in energy with increasing deformation, and are fairly close to the ground state in the strongly-deformed nuclides near $\mathrm{A}=$ 250. The orbitals that are important for fixing the shell corrections near $N=184$ are the $h_{11 / 2}$, $\mathrm{j}_{13 / 2}$ and $\mathrm{k}_{17 / 2}$ spherical states. For each of these spherical orbitals, there is a corresponding deformed orbital whose energy in the $A=250$ region is quite sensitive to one of these spherical states, e. g. the 1/2 - [761] Nilsson orbital that was already identified in ${ }^{251} \mathrm{Cf}$ is quite sensitive to the spherical $j_{13 / 2}$ orbital. The position of the $1 / 2+[880]$ deformed orbital is very sensitive to the $\mathrm{k}_{17 / 2}$ spherical state. According to our calculations, this state should be found at $\sim 1500 \mathrm{keV}$ in ${ }^{25} \mathrm{l} \mathrm{C}$ and should be populated in a one nucleon iransfer reaction using a $\left(\alpha,{ }^{3} \mathrm{He}\right)$ reaction. We calculated signatures for the low-lying states in ${ }^{23} \mathrm{Cf}$ and the calculated energies and signatures are in gond agreement with the observed (d,p) spectrum. We expect to see the high-j states in a $\left(\alpha,{ }^{3} \mathrm{He}\right)$ study.

\section{f. Expluration of Nuclear Energy Surfaces at High Spin in Multi-Dimensional Deformation Spaces (R. R. Chasman)}

One of the major weaknesses of the Strutinsky method is that all deformation modes must be included explicitly in a calculation. With conventional computers and supercomputers. one is 
restricted to a two- or at best three-dimensional Jeformation space for extensive explorations. This means that there may be many interesting nuclear structure features that cannot be found because only a limited region of deformation space is being explored. Recently, massively parallel supercomputers have become (or are becoming) available. These are computers that consist of many nodes; with each node consisting of a CPU and memory. Such a machine is ideally suited for calculations of energy surfaces in multi-dimensional deformation spaces. The MCS Division at Argonne recently obtained such a computer; the IBM SPI computer.

We found many new interesting nuclear structure features by exploring a three-dimensional deformation space that consists of quadrupole, hexadecapole and necking deformation modes. This includes the very extended minima with axis ratios of $2.2: 1$ and $3: 1$ in the $A=180$ mass region. Hopefully, new interesting nuclear structure features can be found by studies of more extended deformation spaces. We modified our programs for calculating energy surfaces using the Strutinsky method and are currently carrying out studies on the SPl.

\section{g. Many-Body Wave Functions (R. R. Chasnian)}

In the past few years, we developed many-body variational wave functions that allow one to treat pairing and particle-hole interactions on an equal footing. by using residual interaction strengths (e.g., the quadrupole interaction strength) as generator coordinates, one gets many different wave functions, each having a different value of the quadrupole moment. These wave functions are particularly useful when one is dealing with a nucleus that has several different minima in the energy surface, such as in the $\mathrm{Hg}$ isotopes where there is a superdeformed minimum in addition to those at small deformation. Because the same basis states are used in the construction of the many-body wave functions for all values of the quadrupole moment, it is possible to calculate overlaps and interaction matrix elements for the many-body wave functions (which are not in general orthogonal) fairly easily. With the product structure of our wave functions, it is possible to include all particle-hole configurations with a fixed value of $\mathrm{J}_{\mathrm{z}}$ in the many-body variational wave functions. The variational wave functions contain a large number of single-particle basis states. In our first calculations of transition matrix elements in the $\mathrm{Hg}$ region, we used a basis consisting of all spherical proton orbitals with $40<\mathrm{Z}<126$ and all spherical neutron orbitals with $70<N<184$. In spite of the large size of this basis, it appears that it is not large enough. We can effectively increase the size of this basis, by constructing basis states that are linear combinations including single-particle states from many higher shells. In this way, we retain the important feature of orthogonality of basis states. We do however give up the orthonormality of our basis functions, for different values of the generator coordinate. In the past year, we added a cranking term to the many-body Hamiltonian and modified the projection procedure to get states of good signature before variation. We are now working on including rather general two-body particle-particle interactions in our code, in addition to the particle-hole terms that are already included.

\section{h. Rotation of Thermally Excited Nuclei (T. Dфssing,* A. Bracco, $\uparrow$ R. A. Broglia, $\dagger$ S. Leoni, $\uparrow$ E. Vigezzi, $\uparrow$ S. Frauendorf, $\neq$ B. Herskind,$\S$ and M. Matsuo )}

It is now a well established experimental fact that deformed rotating nuclei undergo a transicion from having well defined rotational bands to a damped rotational motion at a rather low temperature. It is an open question whether the onset of rotational damping also marks the onset of chaotic motion in the rotational degree of freedom as well as in other degrees of freedom.

*On leave of absence from The Niels Bohr Institute, Denmark, †University of Milano, Italy ‡Institute of Nuclear and Hadron Physics, Dresden, Germany, §The Niels Bohr Institute, Denmark, IYukawa Institute, Kyoto, Japan 
We study rotational damping, on the one hand within a general framework, and on the other hand within a specific model, cranked mean field bands interacting vid a two-body surface- $\delta$ interaction. As the excitation energy is increased, the resulting levels for nuclei around the welldeformed ${ }^{168} \mathrm{Yb}$ display first a region of about 30 unperturbed rotational bands, followed by mixed bands displaying rotational damping. The rotating mean field states are built of independent excitations, thus displaying ordered separable motion. With the surface- $\delta$ residuai interaction, the onset of damping at around $800 \mathrm{keV}$ of thermal energy is accompanied by a gradual onset of chaotic behavior in level distances and transition strengths, which is achieved around $2 \mathrm{MeV}$.

As in many other nuclear phenomena involving thermal excitation, comparison to experiment involves the development of experimental analysis methods and filtering the information from a model through the experimental filters. For this purpose, a simulation code of the $\gamma$-decay cascades through the calculated mixed rotational bands is piesently being developed. Careful studies of the overall shapes of two- and three-dimensional spectra may reveal new information on the spreading width for the coupling of basis rotational bands into the complicated damped rotational states. Fluctuations of two- and three-dimensional spectra may tell how strong the interaction is between the basis bands, and how abrupt the phase-transition is between damped and undamped rotation.

\section{i. Inelastic Excitations in ${ }^{10}$ B (D. Kurath)}

There is generally good agreement between observation of inelastic excitations and the results of shell-model calculations in ${ }^{10} \mathrm{~B}$. However, recent pion scattering from the $J, T=(3,0)$ ground state to the $(3,0)$ state at $4.78 \mathrm{MeV}$ found only about $1 / 4$ of the calculated strength. This is a weak E2 transition (about $0.1 \mathrm{WU}$ ) and it is found to be very sensitive to the wave functions. By forming orthogonal states between the two lower $(3,0)$ states having only a $1 \%$ admixture, the experimental observation can be accounted for. This could be confirmed by measuring the longitudinal form factor in electron scattering. The only other quantity that is seriously affected by this admixture is the unobserved magnetic octupole moment of the ground state which is reduced by $30 \%$ in magnitude.

\section{E. ATOMIC PHYSICS}

Our theoretical program has no permanent staff, but rather a series of visiting theorists. From May 1993 to October 1994 we are fortunate to have M. Amusia from the Joffe Institute of St. Petersburg, Russia, at Argonne as a visiting Argonne Fellow. He provides essential support for the synchrotron radiation-based part of our atomic physics program. In addition, he has played an important role in theoretical discussions concerning the rest of our heavy-ion collisions program.

P. Sigmund (Odense University, Denmark) visited Argonne in January 1993, completing his year as an Argonne Fellow. He completed his work with $\mathrm{H}$. Esbensen, a nuclear theorist in the Physics Division. They studied the collision processes (angular scattering) of fast heavy ions in thin foils.

L. J. Curtis (University of Toledo) and D. R. Beck (Michigan Technological University) continue to collaborate on different aspects of our heavy-ion spectroscopy programs. L. J. Curtis works with us on the lifetime and spectroscopy measurements of highly-charged few-electron ions at the ATLAS accelerator; D. R. Beck works with L. Young on interpretations of the hyperfine structure measurements at BLASE. 
W. Bauer (Michigan State University) and H. Esbensen of the Physics Division theory group, have worked with our group in analyzing the results of our measurements of the fragmentation of fullerenes hy fast heavy ions.

\section{a. Isotope Sputtering (P. Sigmund)}

During the sputtering process, the cross section for knocking off a recoil atom increases with decreasing mass of the recoiling atom. The rate goes as an inverse power law of the mass $\left(\mathrm{M}^{-\mathrm{m}}\right)$. In addition, the cross section for loss of a recoil atom once it is set in motion also depends on the mass, to the same power, independent of the collision partner. It is noted that these two effects produce a sputtering preferentiality proportional to $\mathbf{M}^{-2 m}$, yielding an isotope difference. It is shown that the same proportionality also can be derived from an explicit evaluation of the particle flux in a ternary medium.

\section{b. The Mean Ion Charge in Ar Photoionization near the K Edge (M. Ya. Amusia)}

Different mechanisms were considered describing the variation of the mean charge of the ions produced in photoabsorption by complex multi-electron atoms. It was demonstrated that near the inner-shell threshold, the picture is considerably simplified and the Auger decay of a virtually created (i.e., below its threshold) vacancy is responsible for the experimentally-observed increase of the mean charge. Convenient formulae are presented for this quantity and for the ionization cross section leading to a given charge ion formation. These parameters can be calculated independently or taken from other experiments.

\section{c. Double Electron Ionization in Compton Scattering of High Energy Photons by Helium Atoms (M. Ya. Amusia and A. I. Mikhailov*)}

The cross section of double-electron ionization of two-electron atoms by Compton scattering of high-energy photons was calculated. It is demonstrated that its dependence on incoming photon frequency is the same as that for single-electron ionization. The ratio of "double-to-single" ionization in Compton scattering proved to be energy independent and almost identical with the corresponding value for photoionization. For the He atom it is $1.68 \%$. This surprising result deserves experimental verification.

\section{d. Ionization of Atoms by High-Energy Photons (M. Ya. Amusia)}

The photoionization of atoms by high-energy photons was considered. In this frequency region the cross section and other characteristics of the process are strong, affected by electron shell polarization and rearrangement effects, including that due to inner vacancy Auger decay. In the high-frequency region, the effects of nuclear structure could be important and noticeable, i.e. of virtual or real excitation of the nuclear degrees of freedom and of the Quantum Electrodynamics vacuum.

Ionization accompanied by secondary photon emission (Compton ionization) is analyzed in the considered domain of energies. 


\section{ATOMIC AND MOLECULAR PHYSICS RESEARCH}

This year, Atomic Physics focussed on research programs in the Physics Division in two principal sections: (1) Accelerator-based atomic physics, (2) Synchrotron radiation-based atomic physics.

Atomic physics ressarch is focussed principally on atomic-structure measurements, and within that domain, on precision measurements useful in testing many-body relativistic atomic calculations. We are also studying the dynamics of several different collision systems of fast ions with thin foils and molecules. Much of the experimental work utilizes three heavy-ion accelerators situated within the Physics Division - ATLAS, a high energy (up to $15 \mathrm{MeV} /$ nucleon) heavy-ion accelerator, primarily used for nuclear physics research; the Dynamitron (up to 4-MeV energy); and BLASE (up to 150-keV energy). The latter two are dedicated to atonic physics experiments. Other experimental heavy-ion research utilizes accelerators at other sites. About a quarter of the program utilizes synchrotron radiation at the National Synchrotron Light Source (NSLS) in Brookhaven. This atomic structure program is being developed in anticipation of the completion of the Advanced Photon Source (the APS) at Argonne. This very intense source of high energy X-rays will be available for atomic physics experiments in 1996.

In 1993, the ATLAS-based heavy-ion program included studies of atomic structure through direct wavelength measurements and lifetime measurements of helium-like heavy ions. The experiments are testing new calculations of relativistic and quantum-electrodynamic (QED) inany-body interactions in terms of atomic energy levels and their decay rates. Several new theoretical developments by our group and by physicists at other institutions during the previous year led to the need for these new measurements. We continue close collaboration with atomic theorists (primarily from other institutions - Notre Dame and Lawrence Livermore Laboratory) especially those performing relativistic calculations of few-electron systems. Our normal-incidence and grazing-incidence monochromators, now equipped with position-sensitive detectors to enhance the rate of data collection in spectroscopic measurements, allow us to utilize the pulsed time structure of the ATLAS beam, thereby reducing significantly the neutron- and gamma-ray-induced background levels.

Using highly ionized xenon ATLAS beams we studied the ionization and fragmentation of the fullerene, $\mathrm{C}_{60}$, using a time-of-flight system to detect the ionized fragments. The measurements constitute the highest energy collisional system studied involving fullerene molecules. Collision studies of the dynamic wake potential produced in solids by fast ions have continued, using $\mathrm{HeH}^{+}$ molecular ion beams in experiments at ATLAS and at the Michigan State University Cyclotron.

Synchrotron-based atomic physics measurements continue with joint support from both DOE and funds provided by Argonne Laboratory management, as part of a lab-wide initiative in support of the 7-GeV Advanced Photon Source (APS). The latter is under construction and is expected to be available for experiments at the beginning of 1996. The initial goal is to make atomic structure studies while gaining staff expertise in this exciting new area of $\mathrm{X}$-ray atomic physics. There is strong overlap between the goals of our ongoing ATLAS experiments testing relativistic aiomic structure, and X-ray interactions with relativistic inner-shell electrons of heavy atoms. Thus, we have collaborated with several University and National Laboratory groups in experiments mainly at the Brookhaven National Synchrotron Light Source (NSLS) X-ray ring.

The program of Coulomb-explosion studies of small molecular-ion structures used the Dynamitron accelerator during the summer of 1993. Using a pulsed molecular-ion jet source, the group continued a series of measurements of structures of some cooled light polyatomic ions aimed at investigating large-amplitude nucleon vibrational motions. 
There was limited use of the BLASE accelerator, with the absence of L. Young on leave at JILA, Colorado. Further laser/fast beam measurements of the lifetime of the upper level of the resonance transition in cesium were completed in collaboration with $\mathrm{C}$. Tanner and A. E. Livingston of Notre Dame University.

The theoretical program was boosted by the presence of Miron Amusia, from the A.V. Joffe Institute in St. Petersburg, Russia, who is working primarily with the synchrotron radiation-based program, but who is helpful on all aspects of the atomic program. Other active collaborations continue mostly with other university-based theoretical groups. P. Sigmund of Odense University completed his term as Argonne Fellow with a final one-month :'isit in January 1993. Other active collaborations include W. R. Johnson and J. R. Sapirstein at Notre Dame, L. J. Curtis at Toledo, and D. A. Beck at Michigan Technical University.

\section{A. ATOMIC PHYSICS AT ATLAS}

The narrow energy spread of beams from ATLAS together with the continuous energy variability and capability of operation in a deceleration mode make it an ideal machine for the study of the atomic physics of highly ionized atoms. The recently completed upgrade of ATLAS provides more intense beam lines and an increased range of ion species which further increases the usefulness of ATLAS for atomic physics studies.

Atomic physics experiments at ATLAS have produced some if the most precise determinations of lifetimes of few-electron ions in high-Z $z y /$ stems. These lifetimes are sensitive to higher-order relativistic corrections to the calculations which depend strongly on $Z$. Other spectroscopy programs at ATLAS include precise measurements of transition energies. These experiments test relativistic and radiative corrections to the energy-level calculations as well as correlation effects in the simplest systems where such effects are present. Our collaboration with Notre Dame utilizes ultraviolet spectroscopy to study transitions within the $n=2$ shell of two- and three-electron ions. The NIST group is using $\mathrm{X}$-ray spectroscopy to study transitions in one- and two-electron ions. The Bochum group is studying intercombination transitions in highly charged $\mathrm{Mg}$-like, Al-like and Si-like ions. The ion-atom collision work at ATLAS has concentrated on the study of $\mathrm{C}_{60}$ in collisions with fast xenon $\left(18^{+}\right.$to $\left.35^{+}\right)$ions.

a. Forbidden Transitions in Few-Electron Ions (H. G. Berry, S. Cheng,* R. W. Dunford, D. S. Gemmell, E. Kanter, C. Kurtz, B. J. Zabransky, L. J. Curtis,* and A. E. Livingston $\dagger$ )

The study of helium-lil:e ions at high $\mathrm{Z}$ provides a good means for testing atomic structure calculations in a regime in which relativistic effects and electron-electron correlations are both important. Measurements of the lifetimes of helium-like ions of high $\mathrm{Z}$ provide a means of testing the calculations of wavefunctions. In the past year we have completed a measurement of the lifetime of the $2{ }^{3} \mathrm{P}_{2}$ level in helium-like krypton which is calculated to be $9.5 \mathrm{ps}$. This experiment is part of an effort by our group to develop methods for measuring ultra-short atomic lifetimes in the range 100 fs to $10 \mathrm{ps}$.

In the experiment, we used a double-foil target as the basis for a new method for measuring the lifetimes of short-lived states. The foils were parallel to each other and perpendicular to the beamline velocity. Two forms of the experiment were used. One involved the measurement of the charge state following the two foils and the other involved observation of X-rays downstream of the second foil. Most of the effort in our initial run was devoted to investigating different combinations of target materials and thicknesses using the two different forms of the experiment.

*University of Toledo, + University of Notre Dame 
In the particle form of the measurement, we exploited the preferential loss of excited electrons in the second foil. Hence by changing the foil separation, the charge-state distribution downstream of the foils is changed (Fig. V-1).

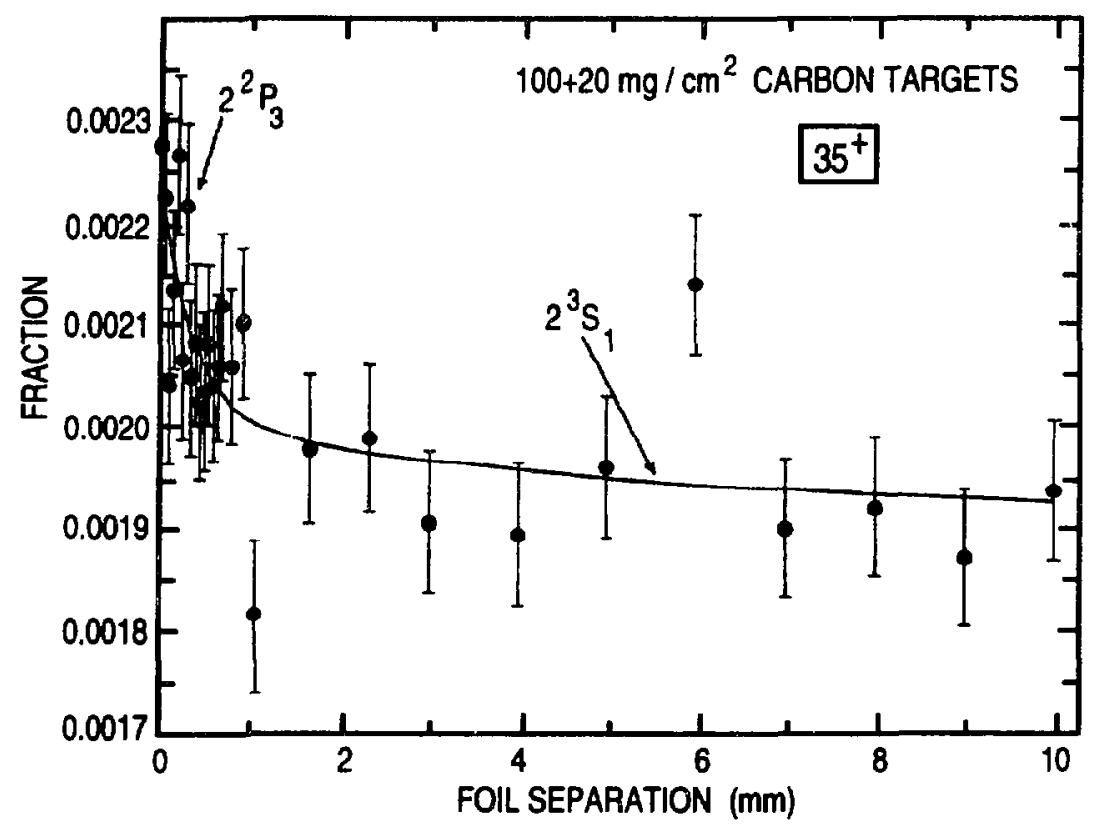

Fig. V-1. Data from the particle form of the two-foil lifetime experiment. The fraction of ions emerging from the target in the 35+ charge state changes as the separation between the foils is changed. These data represent a little over 2 hours of collection time.

In the X-ray form of the measurement, we placed a $\mathrm{Si}(\mathrm{Li}) \mathrm{X}$-ray spectrometer downbeam of the second foil so that it detected photons emitted by beam ions in the region from 5 to $10 \mathrm{~mm}$ downstream of the second foil (Fig. V-2). Photons emitted by beam particles at this distance from the second foil are mostly due to decay of the $2{ }^{3} S_{1}$ level in helium-like krypton. We measured the intensity of the X-rays as a function of the foil separation. We observed a two-component exponential decay with lifetimes corresponding to the $2{ }^{3} P_{2}$ and the $2{ }^{3} S_{1}$ levels (Fig. V-3). Together these two components account for more than $25 \%$ of the $2{ }^{3} \mathrm{~S}_{1}$ line intensity in the $\mathrm{Si}(\mathrm{Li})$ detector. This shows that the production of the $2{ }^{3} S_{1}$ state at the second foil is strongly affected by the population of the excited states of the ions incident on this foil. We are working on a theoretical model to account for these data. Our results indicate that we can produce a clean decay curve using this technique and measure very short lifetimes by looking at $\mathrm{X}$-rays from a long-lived state well down beam line of the target. The measurement determines the lifetime to about $10 \%$ which is close to providing sensitivity to the relativistic corrections. This result involved about two hours of integration time and was limited by counting statistics. In the coming year we plan to repeat the measurement and obtain a result at the few percent level. This would provide a significant test of the relativistic theory. A further by-product of this work will also be the first measurements of excited state charge-changing cross section. 


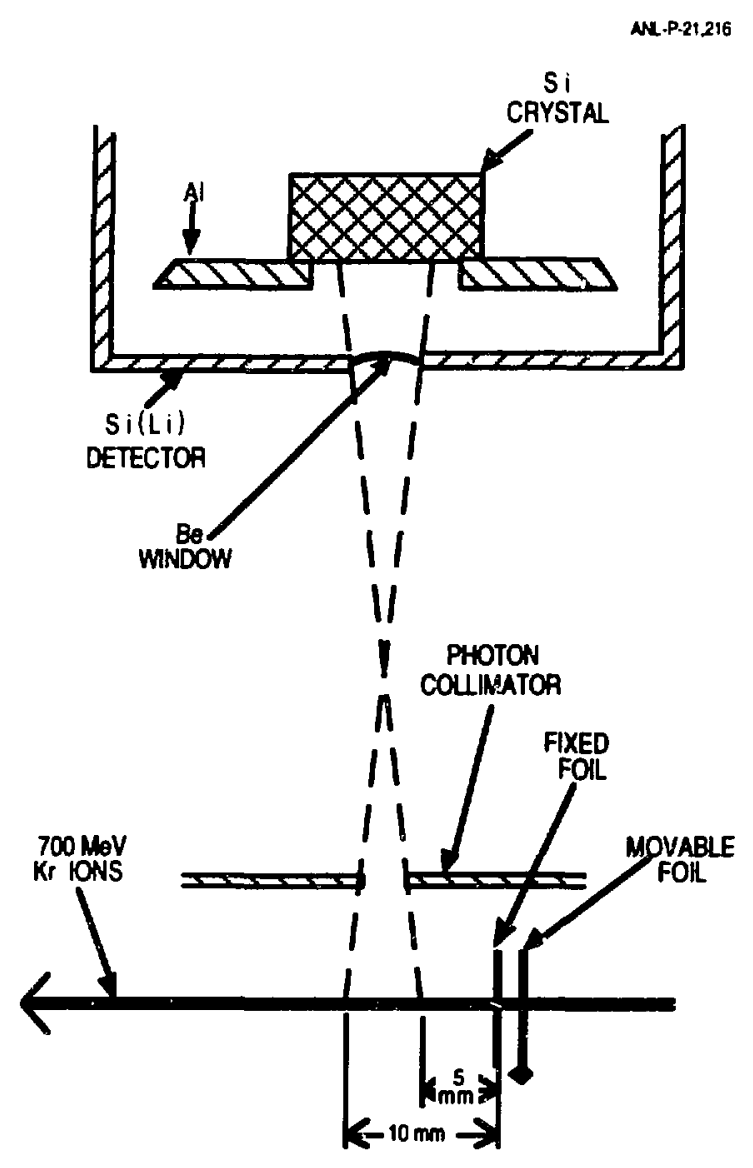

Fig. V-2. Schematic diagram of the X-ray form of the two-foil lifetime experiment.

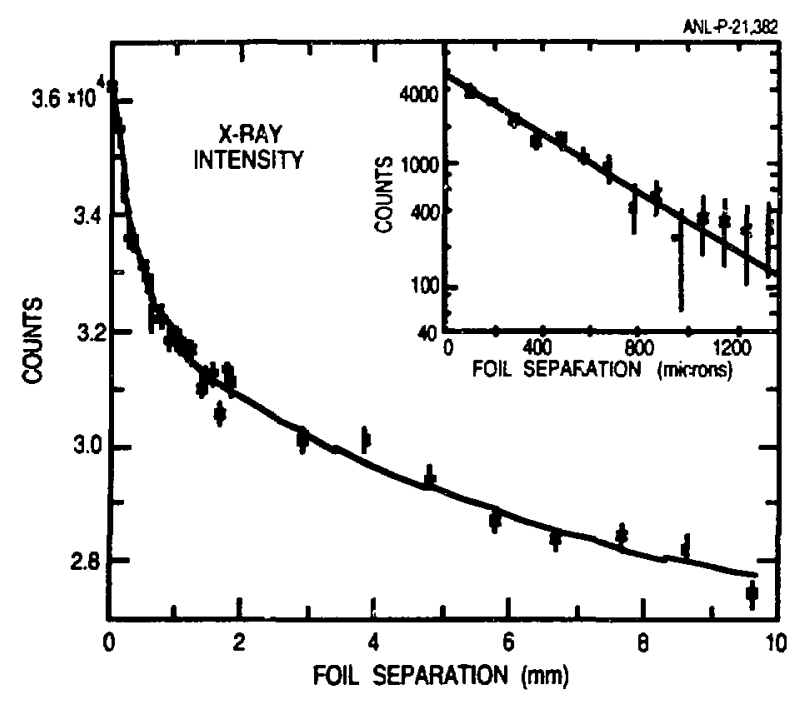

Fig. V-3. Intensity of the MI line in the $X$-ray detector as a function of the separation between the foils in the two-foil target. The solid line is a fit with two $\mathrm{A}_{\mathrm{M} 2}^{\mathrm{H}}$ exponentials and $a$ constant background. The inset is a plot of the decay curve after subtraction of the constant background and the $2^{3} S_{I}$.

b. The Fragmentation of Fullerenes by Fast Heavy Ions (H. G. Berry, S. Cheng,* R. W. Dunford, H. Esbensen, D. S. Gemmell, E. P. Kanter, T. LeBrun, M. A. T'alebian, and W. Bauert)

Atomic collision techniques offer powerful tools for investigating the structure and dynamics of fullerenes. In the past year, we completed a set of experiments in which a $\mathrm{C}_{60}$ (99.5\% pure) vapor target was bombarded by $625-\mathrm{MeV}{ }^{136} \mathrm{Xe}^{35+}$ ions from the ATLAS accelerator. The resulting center-of-mass energies were several orders of magnitude higher than those used previously for studying fullerenes. We found that the collision mechanisms at these high energies and high charge states differ significantly from those seen at lower energies. Positively ionized carbon fragments ranging from single carbon atoms up to $\mathrm{C}_{60^{+}}$were observed using time-of-flight techniques (Fig. V-4.). The measured peaks fall into three categories: 


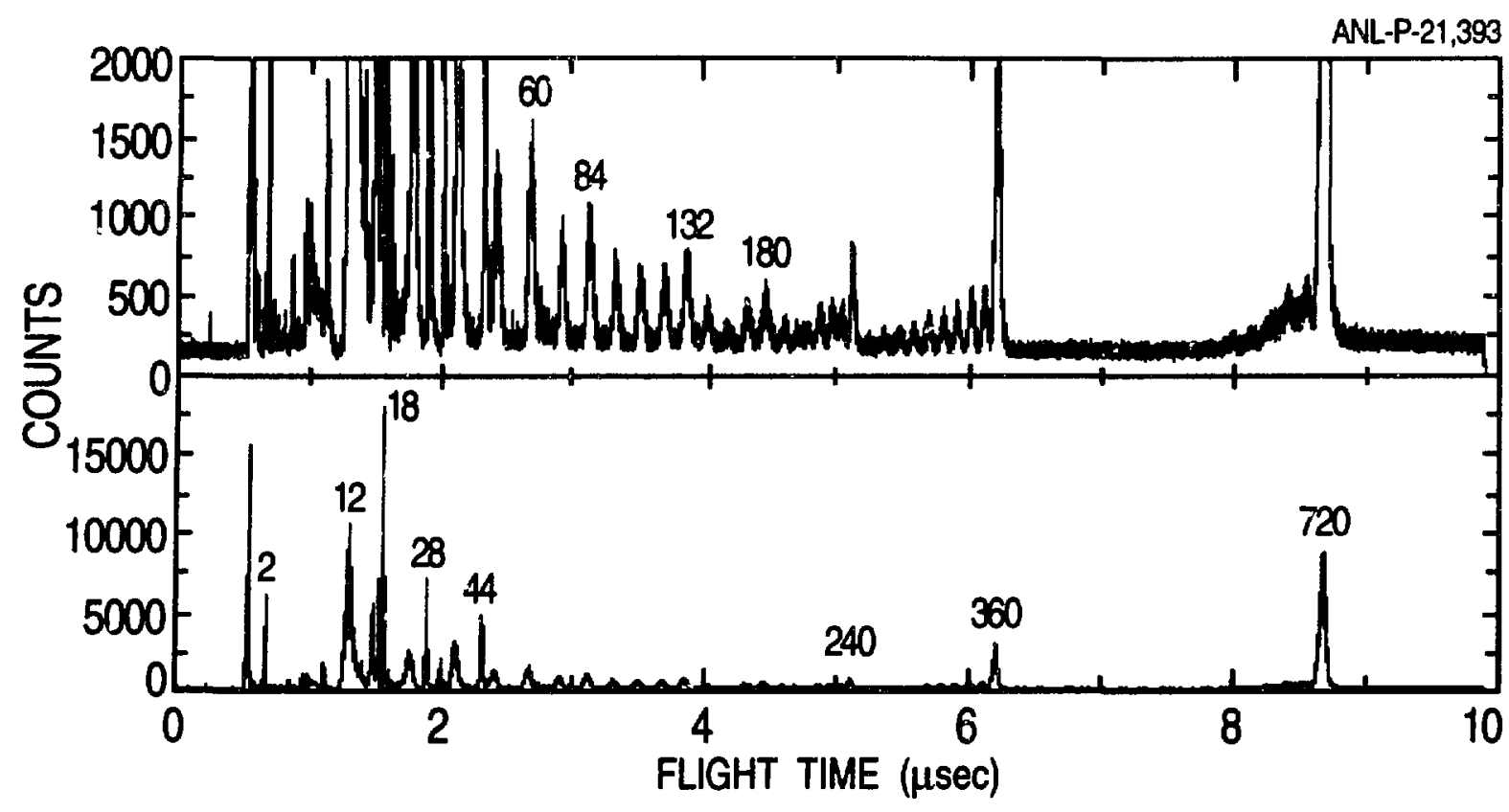

Fig. V-4. Time-of-flight spectrum for positive fragments arising from bombardment of $C_{60}$ by $625-\mathrm{MeV} 136 \mathrm{Xe} 35+$ ions. The numbers given above some of the peaks are the ratios $(M / Q)$ of fragment mass (amu) to charge. The two panels show the same data on different vertical scales.

1) Peaks due to singly, doubly, triply, and (possibly) quadruply ionized $\mathrm{C}_{60}$.

2) "Pair-loss" peaks $\left(\mathrm{C}_{58}{ }^{\mathrm{q}}, \mathrm{C}_{56}{ }^{\mathrm{q}}, \mathrm{C}_{54^{q+}}\right.$, etc.) corresponding to the successive losses of carbon pairs.

3) Peaks corresponding to the sequence of singly charged fragments $\mathrm{C}_{n}{ }^{+}$, with $\mathrm{n}$ assuming all values from 1 to at least 19 . These "multifragmentation peaks" arise predominantly from events in which there is a catastrophic disintegration of the $\mathrm{C}_{60}$ molecule into many small pieces.

We have completed analysis of the two runs conpleted in the past year and have found that we can explain our measurements on the basis of two main processes -1) excitation of the 20-eV giant plasmon resonance in $\mathrm{C}_{60}$ (mostly from large-impact parameter collisions), and 2) multifragmentation arising from closer impact-parameter collisions. The fragment-mass distribution from the second process is explained in terms of a percolation-theory model.

$\overline{\text { *University of Toledo, }} \uparrow$ NSCL, Michigan State University

c. Precision Spectroscopy of the $2 s-2 p$ Fine Structure Transitions in Heliumlike $\mathrm{Ar}^{16+}$ and $\mathrm{Ni}^{26+}$ (H. G. Berry, R. W. Dunford, D. S. Gemmell, E. P. Kanter, C. Kurtz, B. J. Zabransky, A. E. Livingston, ${ }^{*}$ F. G. Serpa, ${ }^{*}$ and K. Kukla*)

At intermediate and high nuclear charge, measurements of the $2 s-2 p$ transition energies provide sensitive tests of many-body relativistic and QED calculations. We are investigating these transition energies at intermediate $Z$ where the experiments are most sensitive to relativistic correlations since the important non-relativistic effect is minimized and transition energies are not dominated by the one-electron Lamb shift as they are at higher $\mathbf{Z}$. 
The current goal of this work is to make a measurement of the $1 \mathrm{~s} 2 \mathrm{~s}{ }^{3} \mathrm{~S}_{1}-1 \mathrm{~s} 2 \mathrm{p}{ }^{3} \mathrm{P}_{0}$ transition energies in helium-like argon and nickel. We use vacuum ultraviolet spectroscopy and positionsensitive detection of foil-excited ion beams at the ATLAS accelerator facility. The final data for the $\mathrm{Ar}^{16+}$ measureinent has been obtained and data analysis is nearing completion. Preliminary runs for the $\mathrm{Ni}^{26+}$ experiment have been done and we expect to complete this measurement in the coming year.

d. Measurement of Transition Energies in Li-Like and He-Like Calcium (H. G. Berry, S. Cheng, R. W. Dunford, D. S. Gemmell, E. P. Kanter, C. Kurtz, J. Suleiman, ${ }^{*}$ B. J. Zabransky, R. D. Deslattes, ${ }^{\dagger}$ P. Indelicato $\left.{ }^{\dagger}\right)$

In a program aimed at testing theoretical calculations of transition energies in two- and three-electron ions we are using a crystal X-ray spectrometer to obtain high-resolution X-ray spectra. The spectrometer observes X-rays formed after electron capture in a gas target. An important aspect of this work is the use of the accel/decel technique in which ions are accelerated, stripped to a oneelectron state, then slowed down and delivered to a gas target where they pick up electrons under single-collision conditions. The deceleration is required in order to obtain adequate cross sections for electron pickup in the gas target. The importance of using a gas target is that single-electron pickup is highly favored and so clean, symmetrical spectral lines, uncontaminated by lines from multielectron pickup, are obtained.

We have completed two preliminary runs for a measurement of the $2 \mathrm{p}-2 \mathrm{~s}$ transition energy in heliumlike $\mathrm{Ca}^{18+}$. Hydrogen-lik- $\mathrm{Ca}$ ions were incident on an argon gas target and the $\mathrm{X}$-rays emitted after electron capture were analyzed with the spectrometer. The preliminary runs point up a need for more intense $\mathrm{Ca}$ beam lines. This has motivated a significant development effort by the accelerator operations group, and they have recently achieved a significant increase in $\mathrm{Ca}$ beam intensity using the ECR source.

*Gradıate Student, University of Illinois, † National Institute of Standards and Technology

e. RTE and REC in Collisions of $\mathbf{U}^{90+}$ Ions on Carbon (R. W. Dunford and Atomic Physics Group at GSI, Darmstadt, Germany)

In the past year we participated in atomic collision experiments at GSI, Darmstadt, Germany. The experiments involved studies of electron capture by highly charged ions in solid targets. We studied the processes of Resonant Transfer and Excitation (RTE) and Radiative Electron Capture (REC). The goal was to test theoretical calculations of atomic structure and collision phenomena. Such calculations are an essential starting point for theoretical models of plasmas and other phenomena with applications to advanced technology applicable to energy-related problems.

We studied REC and RTE with projectiles of helium-like uranium incident on carbon targets. These studies required uranium beams with energies above $100 \mathrm{MeV} / \mathrm{amu}$. At the present time such beams are not available in the United States. The recently completed heavy-ion synchrotron at GSI (SIS) is capable of beam energies of up to $2 \mathrm{GeV} / \mathrm{amu}$ and so our requirements were easily met.

The experiments worked very well and sufficient data were obtained to provide precise determination of the cross sections for both RTE and REC. In addition, the angular distribution of the decay radiation in the case of the REC process was measured. 


\section{B. FAST-ION-BEAM/LASER STUDIES AT BLASE}

During this year we have made precision lifetime measurements in neutral cesium and neutral lithium. The former measurements have been completed on the $6 \mathrm{p}_{1 / 2}$ and $6 \mathrm{p}_{3 / 2}$ states, to provide a precision comparison with calculations at better than the $0.3 \%$ level. This work makes it possible to compare other work with predictions of a nuclear anapole inoment in cesium. The measurements in lithium are aimed at resolving a long-standing 15-year difference between precise theory and experiments for the $2 s-2 p$ transition.

a. Precision Lifetime Measurements by Single-Photon Counting (L. Young, W. T. Hill JI,* S. J. Sibener, † S. Price, $\ddagger$ C. E. Tanner,§ C. E. Wieman, I and S. R. Leone()

There is renewed interest in the accurate measurement of lifetimes of excited states in alkalis in order to test the $a b$ initio theory which is used to interpret atomic parity non-conservation measurements in cesium. While it is often assumed that the fast-beam laser method, described below, yields the most accurate lifetimes, we have demonstrated that an alternative technique, timecorrelated single-photon counting, is capable of achieving comparable accuracy. Given that the two techniques have completely different systematic errors associated with them, and that the final accuracy is dominated by systematics, these measurements provide an imiportant check of earlier results.

Briefly, in the single-photon counting technique a fast pulse from a laser selectively excites the state of interest at $\mathrm{t}=0$. This starts a clock (time-to-amplitude converter, TAC) which is stopped by the arrival of a fluorescence photon. The time interval is binned in a multichannel analyzer (MCA) and the entire sequence repeated until the required statistics have been attained.

*University of Maryland, †University of Chicago, $\ddagger$ University College, London, §University of Notre Dame, qUniversity of Colorado

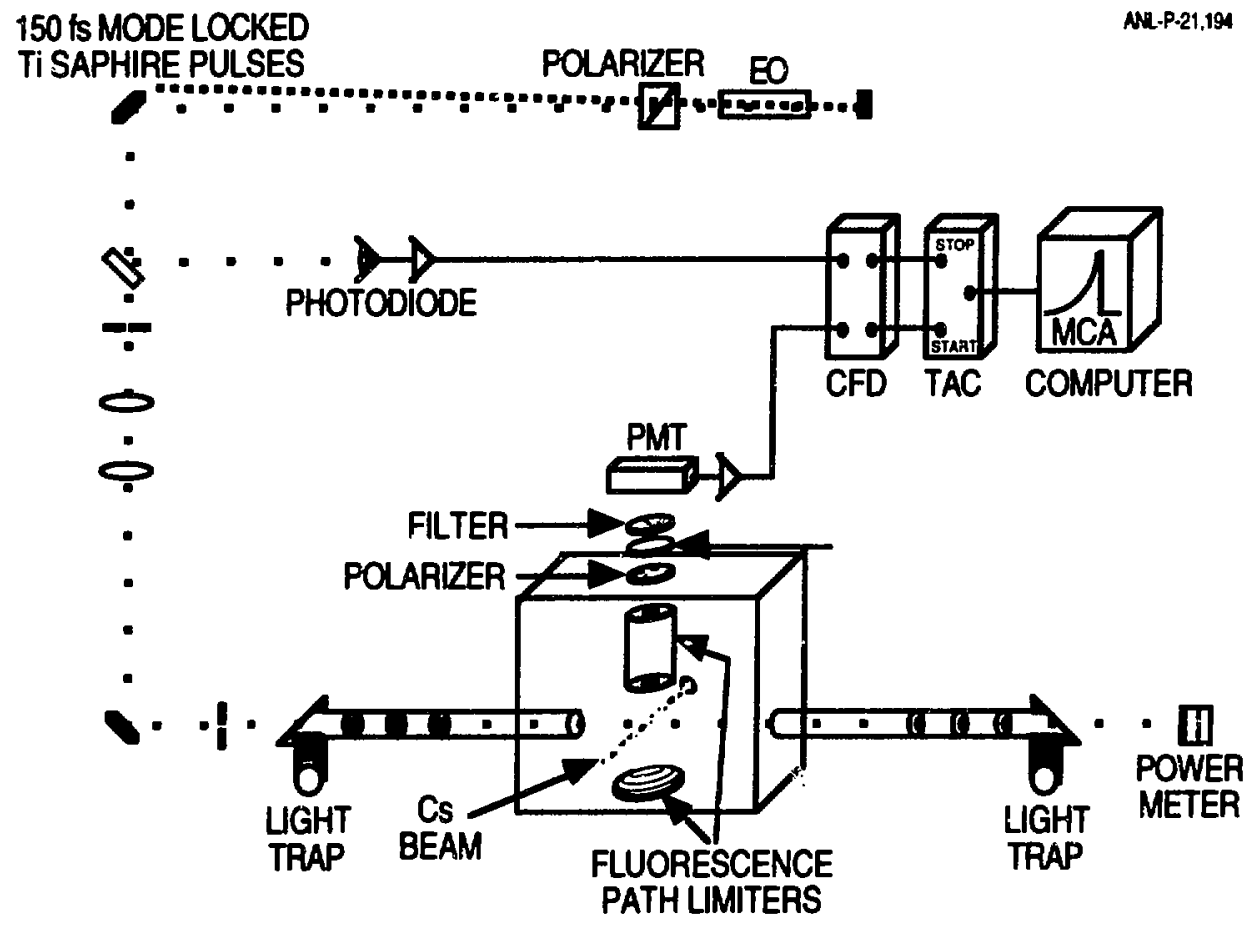

Fig. V-5. Schematic of the experimental arrangement. See text for detailed description. 
In this particular experiment (Fig. V-5) performed at JILA, the output of a self-mode-locked Tisapphire laser $(\Delta t \sim 150 \mathrm{fs}$, repetition rate $\sim 76 \mathrm{MHz})$ is used to excite the lowest-lying odd parity levels $\left(6 \mathrm{P}_{1 / 2}, 6 \mathrm{P}_{3 / 2}\right)$ of $\mathrm{Cs}$ in a well-collimated atomic beam. An electro-optic (EO) switch is used to select laser pulses at the desired repetition rate, 4.4 and $5.0 \mathrm{MHz}$, respectively, for the $6 \mathrm{P}_{1 / 2}(\tau \sim$ $35 \mathrm{~ns}$ ) and $6 \mathrm{P}_{3 / 2}(\tau \sim 30 \mathrm{~ns}$ ) states. A double-pass configuration through the EO was required to achieve the desired extinction of the rejected pulses $(\sim 0.2 \%)$. The fluorescence was viewed at right angles to the laser and atomic beam propagation through a polarizer and filters. The fluorescence from the $6 \mathrm{P}_{3 / 2}$ state was detected with the polarizer set at the magic angle $\left(54.7^{\circ}\right)$ with respect to the electric field polarization axis of the laser in orde- io eliminate hyperfine quantum beats (Fig. V6 ). For the $6 \mathrm{P}_{1 / 2}$ state the polarizer was removed. The time scale was mapped directly onto the MCA by deliberate leakage of afterpulses through the EO and in situ scattering into the detection stack from a pinpoint rotated under vacuum to the atomic beam/laser interaction volume. The actual repetition rate of the laser was determined by standard frequency counting techniques. Other systematic effects due to radiation trapping, collisions, pulse pileup, Zeeman quantum beats, nonlinearities in the TAC/MCA were measured and/or estimated, and included in the final error budget.

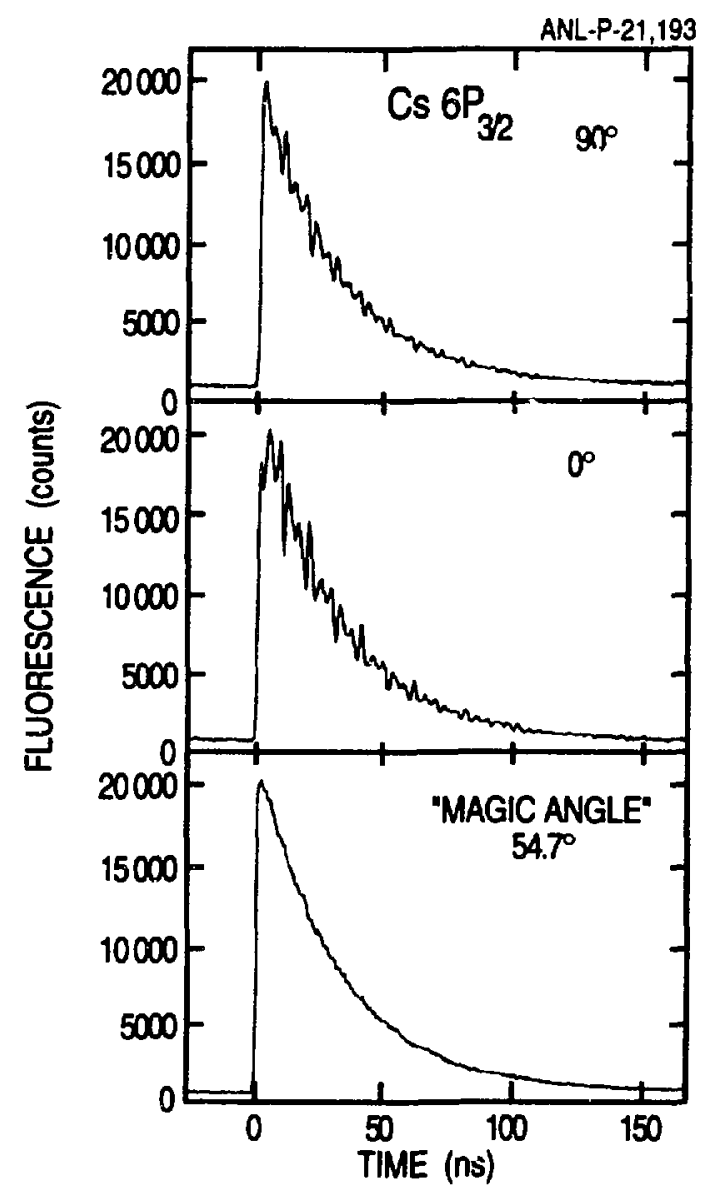

Fig. V-6 Elimination of hyperfine quantum beats from fluorescence from $C s 6 P_{3 / 2}$ state. Top. middle, and lower panels show fluorescence from the $6 P_{3 / 2}$ state with the detection polarizer oriented at $0^{\circ}, 90^{\circ}$ and $54.7^{\circ}$ with respect to the electric field polarization vector of the laser. $54.7^{\circ}$ corresponds to the "magic angle" where $3 \cos ^{2} \theta-1=0$.

The lifetime for the $6 \mathrm{P}_{1 / 2}\left(6 \mathrm{P}_{3 / 2}\right)$ states was measured to $\sim 0.2 \%(0.3 \%)$. The lifetimes are shorter, but in reasonable agreement with the fast-beam laser results. They exhibit the same $\sim 1 \%$ systematic offset from $a b$ initio results (experimental lifetimes are longer than $a b$ initio) that was initially revealed for the lighter alkalis. 
With the experience gained by this initial measurement, it is clear that this technique can be extended to measure lifetimes with $\$ 0.1 \%$ preision and provide benchmark values for testing atomic theory calculations.

\section{b. Hyperfine Structure Studies of Zr II (L. Young, C. A. Kurtz, D. R. Beck,* and D. Datta*)}

Recent advances in $a b$ initio atomic theory have spurred a renewed interest in the atomic hyperfine structure (hfs) as a test of the wavefunction near the nucleus. In combination with oscillator strength measurements and energy level data, these measurements provide very stringent tests of the calculations. While much of the activity has concentrated on alkali-like systems (with a single valence electron), we have pursued studies of systems with two, three and four valence electrons. The development of relativistic configuration-interaction methods by $D$. R. Beck to predict the hfs from first principles has been a direct outgrowth of our earlier measurements and observation that standard (independent particle) multiconfiguration calculations fail dramatically in the prediction of magnetic contact interactions (primarily due to polarization of the core).

As an extension of our previous studies of transition metal ion hyperfine structures (hfs), we have studied the three valence electron ions $\mathrm{Ti}$ II and $\mathrm{Zr}$ II. ${ }^{91} \mathrm{Zr}$ II was chosen both to test the magnitude of relativistic contributions compared to the Ti II case, and as a testing ground for the newly-developed relativistic computational algorithms. Experimentally, hyperfine structures in 11 levels arising from the metastable $4 d^{3}$ and $4 d^{2} 5$ s configurations were measured using the laser-rf double resonance method. The hfs $A$ and $B$ constants were measured to 4 and $11 \mathrm{kHz}$ nrecision, respectively. Less precise values for hfs constants were derived from optical spectra for 9 upper levels in the $4 d^{2} 5 p$ configuration.

Theoretically, the many-body problem was treated with a relativistic configuratiun interaction (RCI) approach. Since one is starting with relativistic wavefunctions, all levels with a given $J$ must be calculated simultaneously. Two $J s$ were selected for the initial study, $J=3 / 2$ and $J=1 / 2$. The calculations start with a zeroth order wavefunction which is a multiconfigurational Dirac-Fock (MCDF) solution. Many-body effects are then included by allowing configurationally single and double excitations from the zeroth-order function. By adding such excitations in a systematic fashion, the various many-body effects can be distinguished from one another. The calculated wavefunctions should predict simultaneously the measured level energy, hfs A and B constants. For the $\mathrm{J}=3 / 2$ levels, the average energy discrepancy for the ten lowest roots was $0.087 \mathrm{eV}$ and the average discrepancy with the three measured $A$ values was $9.2 \%$. For the $J=1 / 2$ levels, the average discrepancy was $0.050 \mathrm{eV}$ in energy and $31.8 \%$ for the $\mathrm{A}$ values. This represents a remarkable improvement over the independent particle Dirac-Fock values for which the discrepancies in the $A$ value: were $88 \%$ and $1 \% 6 \%$ for the $J=1 / 2$ and $3 / 2$, respectively. A value of $0.257(0.013) \mathrm{b}$ is obtained from a compariso: 1 of the experimental B values and theoretical matrix elements. In addition, the calculations confirm a previous report that the level at $17614.7 \mathrm{~cm}^{-1}$ reported in Moore's Atomic Energy Levels, Vol. II is spurious.

*Michigan Technological University

c. Laser-rf Double Resonance Studies of Hyperfine Structure in V II (L. Young, C. A. Kurtz, S. Hasegawa, ${ }^{*}$ G. Matous, † and D. R. Beck $\ddagger$ )

We next studied the first row transition metal ion with four valence electrons, V II. Using the laser-rf double resonance method, we measured the hfs in 4 levels arising from the $3 \mathrm{~d}^{4}$ and $3 \mathrm{~d}^{3} 4 \mathrm{~s}$ configurations. Because of the very large nuclear magnetic moment in $51 \mathrm{~V}$, it was necessary to frequency double the output of the radiofrequency synthesizer $(<2.4 \mathrm{GHz})$ in order

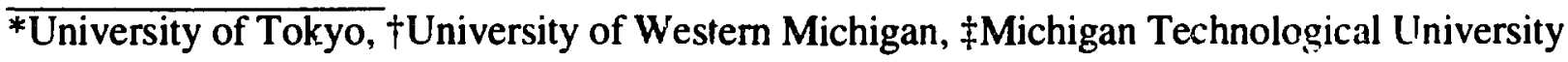


to reach theresonances. Even so, some resonances were not within reach $(<4.8 \mathrm{GHz})$, and hfs of these states was only determined through optical spectra. Since the doubled output is not pure, i.e. contains both fundamental and second harmonic, it was possible to observe two-photon rf transitions. Of the four levels studied using the laser-rf double resonance method, two arose from the $3 d^{3} 4 s$ and two from the $3 d^{4}$ configuration. In addition, hfs from six other levels was measured using optical spectroscopy. Typical precisions for the rf measurements were $5 \mathrm{kHz}$ and $10 \mathrm{kHz}$ for A and B, respectively. Many-body calculations are currently underway.

\section{d. Hyperfine Structure Studies in Nb II (L. Young, C. A. Kurtz, S. Hasegawa,*} D. Datta, $†$ and D. R. Berk†)

The next system studied with four valence electrons was $\mathrm{Nb}$ II. Similar to ${ }^{51} \mathrm{~V}$, the ${ }^{91} \mathrm{Nb}$ isotope has a large nuclear magnetic moment, $6.167 \mu_{\mathrm{N}}$, and thus required frequency doubling of the rf output. In addition, some of the optical pumping transitions were very weak, with oscillator strengths of $10^{-4}$. This motivated us to install a photodiode detector near a beam profile monitor (rotating wire) in order to ensure optimum laser-ion beam overlap in the optical pumping region. We measured the hfs of the ${ }^{3} F_{2,3,4}$ states in the $4 d^{4}$ configuration using the laser-rf double resonance technique. Lower precision optical measurements have been made on the $4 d^{3} 5 p{ }^{3} D_{1,2}$ levels. The $3 d^{4}{ }^{3} F_{3,4}$ levels show evidence of second-order hyperfine structure.

Many-body calculations are underway for the $\mathrm{Nb}$ II, $\mathrm{J}=2(\mathrm{~d}+\mathrm{s})^{4}$ states. In order to accommodate the more complex four-valence electron systems, substantial overhaul of the RCI codes was necessary. The calculations for the four-electron systems now include up to 4000 vectors (as opposed to the 1000 vectors for the three-electron systems). At this point, the following correlation effects have been included: (1) single- and double-valence shell excitations from all reference configurations, (2) $4 p \rightarrow v f$, (3) $4 p^{2} \rightarrow 4 d^{2}+4 d v d$, (4) $4 s \rightarrow 4 d+5 s$, (5) $3 p \rightarrow v p,(6)$ $4 p 4 d \rightarrow$ vpvd and (7) $4 \mathrm{p} \rightarrow$ vp. With these included, the average energy error for the bc:tom ten $\mathrm{J}=2$ levels is $0.053 \mathrm{eV}$. The prediction of these $\mathrm{RCI}$ calculations for the A-value of the ${ }^{3} \mathrm{~F}_{2}$ level is $50 \%$ discrepant with the experimental value, compared with $87 \%$ for independent particle DiracFock calculations. It is hypothesized that more correlation effects are needed to improve the agreement with the experimental values.

\section{*University of Tokyo, $†$ Michigan Technological University}

\section{e. Precision Lifetime Measurements of the $6 p$ Levels in Cesium ( $\mathrm{H}$. G. Berry, C. Kurtz, C. Tanner, * A. E. Livingston, ${ }^{*}$ R. Rafac, ${ }^{*}$ F. G. Serpa,* and K. Kukla*)}

The best tests of parity violation in atomic physics are obtained from studying forbidden transitions in neutral cesium. In order to compare directly with weak neutral current interaction theory, certain other atomic parameters in cesium are needed. The most important of these include the oscillator strengths of the $6 s_{1 / 2}-6 p_{1 / 2,3 / 2}$ transitions. Hence we have attempted precision fast-beam measurements at the BLASE facility of the respective decay lifetimes. A beam of cesium ions from BLASE at $50 \mathrm{keV}$ is neutralized in a rubidium charge-exchange cell, and then interacts with a perpendicular laser beam (from a stabilized diode laser) tuned to one of the $6 \mathrm{~s}-6 \mathrm{p}$ transitions. We measure the intensity decay curve of the fast neutral beam with a movable detector.

Our initial work of 1992 was accurate to better than $1 \%$, which is better than the present best experimental tests of the weak interaction, and establishes that the many-body theory for the oscillator strength is accurate to about $0.5 \%$. Following up our initial work, we have made significant reductions in many of the systematic errors of these earlier measurements. These have

*University of Notre Dame 
resulted in an improvement in our precision of more than a factor of 2 . Although the analysis is not yet complete, the results agree with our previous measurements, and will provide a basis for future weak interaction measurements and calculations in cesium. These latter are aimed at the first clear observation of a nuclear anapole moment.

f. Precision Lifetime Measurements of the 2p Levels in Lithium (H. G. Berry, C. Kurtz, C. Tanner, ${ }^{*}$ A. E. Livingston, ${ }^{*}$ and R. Rafac*)

The fast-beam laser techniques utilized above for cesium have also been applied to make measurements of the $2 \mathrm{~s}-2 \mathrm{p}$ resonance transitions in neutral lithium. Although the technique is similar, this atom produces a few uew complications. The wavelength at $6708 \AA$ requires a dye laser which has inherently lower intensity stability compared to the diode laser; the decay curve of similar lifetine is much longer because of the relative lightness of the lithium atom. Initial results have been obtained and further measurements are in progress.

The oscillator strengths of these transitions in lithium pose a very different theoretical challenge from the cesium measurement; the three-electron lithium atom is one of the simplest atomic systems available and is a testing ground for the best atomic structure calculations. In the past 15 years there were many precise calculations of this oscillator strength. In addition, a very precise laser fast-beam lifetime measurement by Gaupp and Andrä (Berlin) has been the standard between this measurement and the best theories. Hence the need for this new, independent and precise measurement.

*University of Notre Dame

\section{COULOMB-EXPLOSION EXPERIMENTS}

The Coulomb-explosion program concentrated on the application of CEI imaging to the study of large-amplitude nuclear motions in several small polyatomic molecules.

Reinvestigation of protonated acetylene with our improved apparatus demonstrated the groundstate geometry to be highly fluxional with unexpected out-of-plane proton migration.

\section{a. Study of Extended Wake Effects Induced by Fast Ions Traversing Solids (M. P. Carpenter, R. W. Dunford, D. S. Gemmell, T. J. Graber,* R. V. F. Janssens, E. P. Kanter, J. A. Nolen, and B. J. Zabransky)}

We exploited the existence of a high-energy $\mathrm{HeH}^{+}$beam at the National Superconducting Cyclotron Laboratory to study the details of long-range electronic "wakes" induced in solids by the passage of swift charged particles. A diatomic molecular-ion projectile undergoing a "Coulomb explosion" provides a good probe of these wake effects since the wake forces calise significant changes in the alignment of the internuclear vector joining the two separating fragments. A wake represents the collective electron response of the solid to the passage of a fast ion. It is thus directly connected with the excitation of plasmons. The wake "wavelength" is the distance the ionic projectile travels in one plasmon oscillation period and lies typically in the range from about 15-300 Angstroms for ions with energies in the range of about 1-50 MeV/nucleon. Almost all Coulomb-explosion experiments thus far performed with solid targets have only been sensitive to the wake over distances of about 1 Angstrom. In our experiment, we used a double-foil target to extend the studies out to distances of several wake wavelengths. We have completed several runs of this experiment and are now in the process of analyzing the data.

\footnotetext{
*University of Illinois
} 


\section{b. Anomalous Nuclear Delocalization in Protonated Acetylene (T. Graber,* E. P. Kanter, Z. Vager, and D. Zajfman $\dagger$ )}

One of the earliest Coulomb Explosion Imaging (CEI) studies was that of protonated acetylene. Carried out in 1986 with the MUPPATS detector, that experiment was necessarily limited to studying the mean geometry of molecules created in the relatively "hot" electron-impact ion source. At that time, we found a bridged-planar mean geometry which was later verified by both theoretical and spectroscopic investigations (Fig. V-7). Those works, however, seemed to indicate a highly fluxional molecule and it was suggested that large-amplitude proton-planar migration could explain some of the anomalies of the infrared spectrum. We have now reinvestigated this molecule with our improved CEI apparatus and find a surprisingly different result.

With the combined MUPPATS/SAM detectors, we are now able to separate all of the fragments resulting from the Coulomb explosion of $\mathrm{C}_{2} \mathrm{H}_{3}{ }^{+}$and study departures from the mean geometry of the ensemble. Furthermore, with supersonic expansion in the ion source, we were able to cool vibrational motions. We find that when averaged as the old unresolved data was, we again see the bridged planar geometry. However, when we abandon averaging over coordinates and investigate the full correlation between angular degrees of freedom (Fig. V-8), we find instead a dynamic nonplanar structure (Fig. V-7) which differs markedly from previous findings and challenges the Born-Oppenheimer based $a b$ initio treatments and previous spectroscopic results.

*University of Illinois, + Weizmann Institute of Science

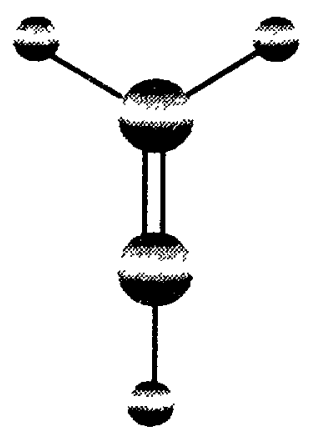

(a)

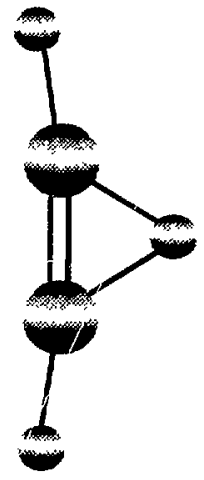

(b)
AN-P-21,126

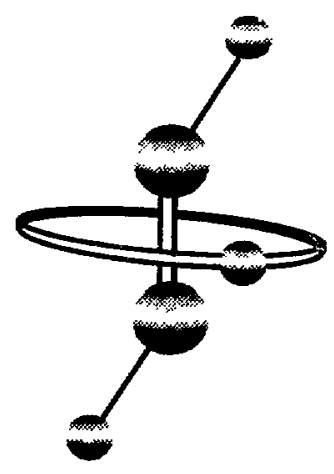

(c)

Fig. V-7. Schematic views of: (a) the classical structure; (b) the bridged structure; and (c) the dynamic non-planar configuration. 


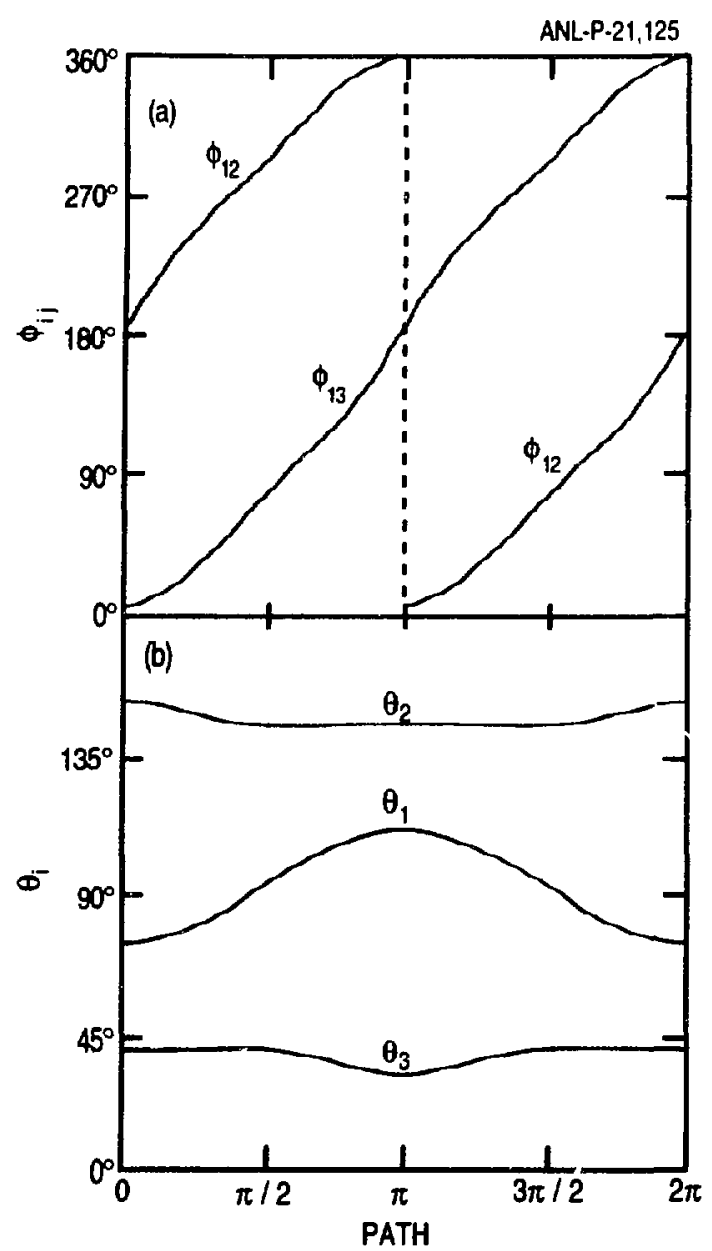

Fig. V-8. (a) The azimuthal angles $\phi_{12}$ and $\phi_{13}$ and (b) the polar angles $\theta_{1}, \theta_{2}$ and $\theta_{3}$ along the most probable path in the measured density demonstrating the reaction path for proton migration. The abscissa represents a phase angle along the reaction path.

c. Bending Vibrations in $\mathbf{C O H}_{\mathbf{n}}{ }^{+}$Ions (T. Graber,* E. P. Kanter, Z. Vager, and D. Zajfman $\dagger$ )

We have investigated several molecules of the form $\mathrm{COH}_{n}{ }^{+}$including $\mathrm{HCO}^{+}, \mathrm{H}_{2} \mathrm{CO}^{+}$, and $\mathrm{CH}_{3} \mathrm{OH}^{+}$. All of these molecules were found to be consistent with theoretical predictions as well as spectroscopic studies where available. In particular, $\mathrm{HCO}^{+}$exhibited a highlyrigid linear structure. With our vibrationally cooled sample of molecules, we saw no evidence for the $\mathrm{COH}^{+}$isomer. Preliminary analysis seems to indicate that the width of the bending amplitude is in good agreement with the spectroscopic measurements. Similarly, our study of the methanol ion showed a single well-defined structure with a rigid cis conformation.

In the case of $\mathrm{H}_{2} \mathrm{CO}^{+}$, we found two very different structures depending upon the method of production in the ion source. When prepared from paraformaldehyde expanded in $\mathrm{Ar}$, we find a rigid formaldehyde structure. In sharp contrast, when the beam was produced from the expansion of methanol in an Ar buffer, we see an equally rigid trans $\mathrm{HCOH}^{+}$ structure. In each case, we find a pure ensemble lacking evidence of the other isomer. These results appear to be consistent with recent theoretical work which shows a large barrier separating two deep wells.

*University of Illinois, $†$ Weizmann Institute of Science 


\section{ATOMIC PHYSICS AT SYNCHROTRON LIGHT SOURCES}

The research program in atomic, molecular, and optical physics with X-rays complements the program of accelerator-based atomic physics research within the Division. Bcth programs provide research in atomic structure as a primary focus. The synchrotron radiation work also provides studies of the fundamental characteristics of X-rays and their interaction with matter, and the advantages these characteristics bring to experimental investigations. Much of the current experimental work takes place at the X24A beam line at the National Synchrotron Light Source (NSLS) at Brookhaven National Laboratory. These activities are expected to lay the foundation for the Physics Division's future research in AMO at the Advanced Photon Source (APS) at Argonne. We are part of the BESSRC (Basic Energy Sciences Synchrotron Radiation Facility) collaboration at the APS to establish a group of beam lines, one of which will be used for AMO physics. The work involves design and development of these beam lines to be ready for the APS opening for experiments in early 1996.

The group operates the X24A NSLS beam line jointly with the Quantum Metrology Division of NIST as part of the Participating Research Team. This agreement provides the group with priority access to this state-of-the-art dedicated facility for research in AMO physics with synchrotron radiation.

The program exploits the unique characteristics of $\mathrm{X}$-rays for studies of atomic structure and dynamics, especially for the inner-shell electrons of heavy and medium-heavy systems. The X-ray photons are especially useful for probing correlation, relativity and quantum electrodynamics in heavy many-electron systems. In addition, the relative simplicity of atoms provides a testing ground for studies of the basic interactions of X-rays with matter, and for comparison to similar phenomena in more complex systems, such as condensed matter or macro-molecules.

The measurements being developed utilize diverse experimental techniques, including X-ray absorption spectroscopy, high-resolution X-ray emission spectroscopy, low-resolution X-ray fluorescence spectroscopy, Auger electron spectroscopy, ion trap studies, ion time-of-flight spectroscopy, and ion-photon coincidence studies.

We will host a workshop on atomic physics with the new generation high-energy synchrotron light sources, to be held at the end of April 1994. It will be principally a US-Japan Seminar, the third of the series, held jointly with the Japanese groups currently making parallel efforts towards establishing atomic physics programs with their SPring8, 8-GeV light source. However, European participation is also expected. We held a workshop in October 1993 on double-photoionization of helium at high energies. A proceedings is in publication.

\section{a. X-Ray Absorption Spectroscopy of Inner-Shell Double Photoionization in}

Argon (Y. Azuma, H. G. Berry, P. L. Cowan, D. S. Gemmell, T. LeBrun, T.Sekioka,* J. Suleiman, $\dagger$ and $M$. Westerlind $\ddagger$ )

We studied multiple excitation of the KM and $\mathrm{KL}$ systems in argon at the X24A beam line at the NSLS. The experiment uses a standard low-pressure absorption chamber filled with argon. The signals are obtained from a PIN dicde located after the chamber, normalized to an initial ion chamber signal. Relativistic Hartree-Fock calculations (the GRASP II code) by one of us (J. Suleiman) enable us to identify most of the structures. Many of these identifications do not agree with earlier assignments from previous lower-resolution experiments. Our excellent signal-tonoise ratio enables us to pinpoint very weak features in the spectra.

*Himeji Institute of ' 'echnology, †University of Illinois, $\ddagger$ University of Tennessee 
b. The X-Ray Photoabsorption Spectrum of Potassium and Rubidium near the K Edge (Y. Azuma, H. G. Berry, P. L. Cowan, D. S. Gemmell, J. Suleiman,* and T. LeBrun)

Initial experiments utilized a standard heat pipe at the X24A beam line at the NSLS to study the Kedge absorption spectrum in atomic potassium vapor. We constructed an improved heat pipe with greater absorption-length stability over time periods of up to a week to study very weak features. We studied the photoabsorption spectra of potassium at a few hundred volts above the K-edge in the photon energy range 3600 to $3650 \mathrm{eV}$. Using the GRASP II multiconfigurational Dirac-Fock code, we made preliminary identifications of most of the peaks observed at the $\mathrm{K}$ and $\mathrm{KM}$ edges. We are able to produce a theoretical spectrum for the $K$ edge which agrees very well with the experimental results by including an experimental width and calculated oscillator strengths, plus an arctangent function to account for the ionization threshold. Initial absorption measurements were also made near the $\mathrm{K}$ edge in rubidium. We made a systematic comparison of these alkali spectra with those of the corresponding closed-shell rare gases obtained previously at X24A and elsewhere. There is a significant enhancement of the $4 p$ (in potassium) and the $5 p$ (in rubidium) resonances compared to the argon and krypton spectra (Fig. V-9). More quantitative comparisons are in progress.

*University of Illinois

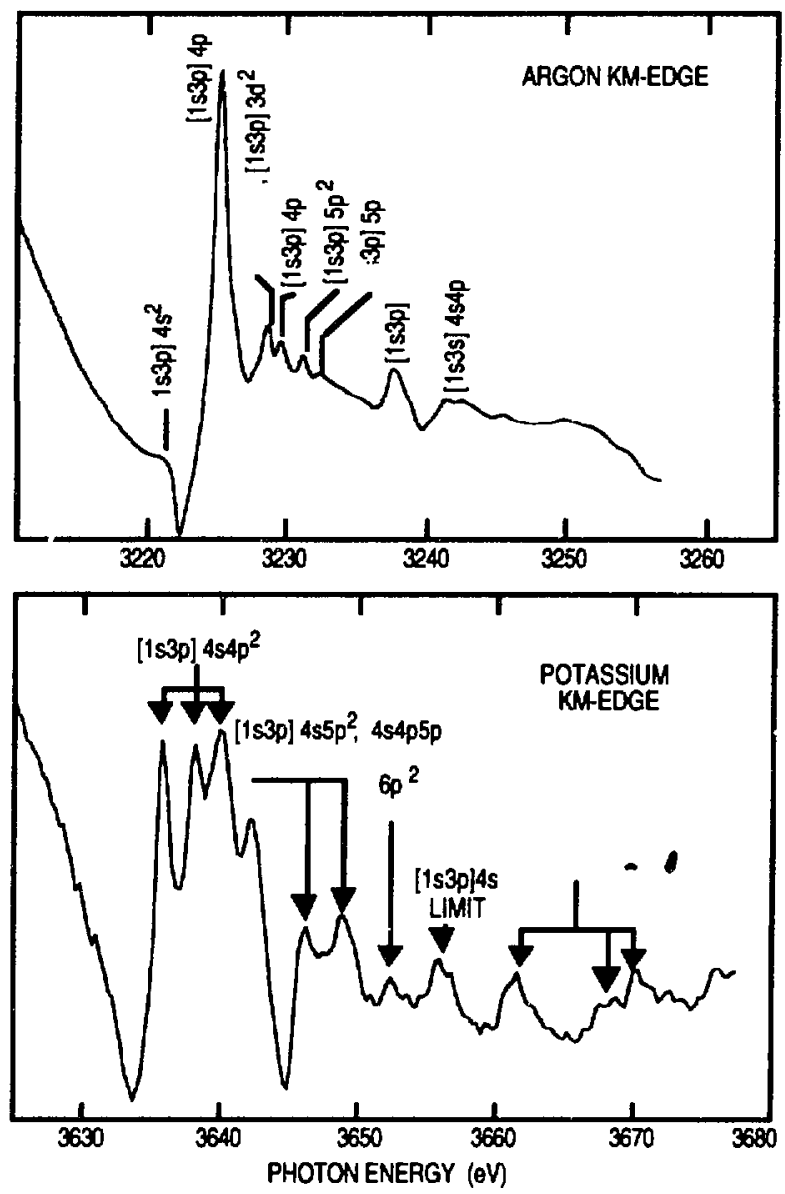

Fig. V-9. Comparisons of the KM satellite structures in the absorption spectra of argon and potassium. Our proposed identifications are given (based on relativistic Hartree-Fock calculations). 


\section{c. Photoabsorption in Helium at 2- to 14-keV Photon Energies ( $Y$. Azuma, H. G. Berry, D. S. Gemmell, T. LeBrun, M. Westerlind,* I. A. Sellin,* J. Woicik, † and J. Kirkland $\ddagger$ )}

We measured the total attenuation cross section of helium for photons in the energy range of 2 to $14 \mathrm{keV}$ (Fig. V-10). Measurements up to $5 \mathrm{keV}$ were made at the X24A beam line, and measurements at energies from 4 to $8 \mathrm{keV}$ were made at the X23B beam line. At these energies, the photoionization cross section is rapidly decreasing, so that Compton scattering dominates at the highest energies. We have analyzed our results in terms of older compilations, and in terms of recent cross-section calculations and recent measurements of the single-to-double photoionization ratio. The measured cross sections are close to the calculated cross sections for Compton scattering and photoionization. We are able to distinguish the contributions of the two effects. We held a two-day workshop at Argonne in October 1993 on this topic.

*University of Tennessee, $\uparrow$ National Institute of Standards \& Technology, $\ddagger$ SFA, Inc.

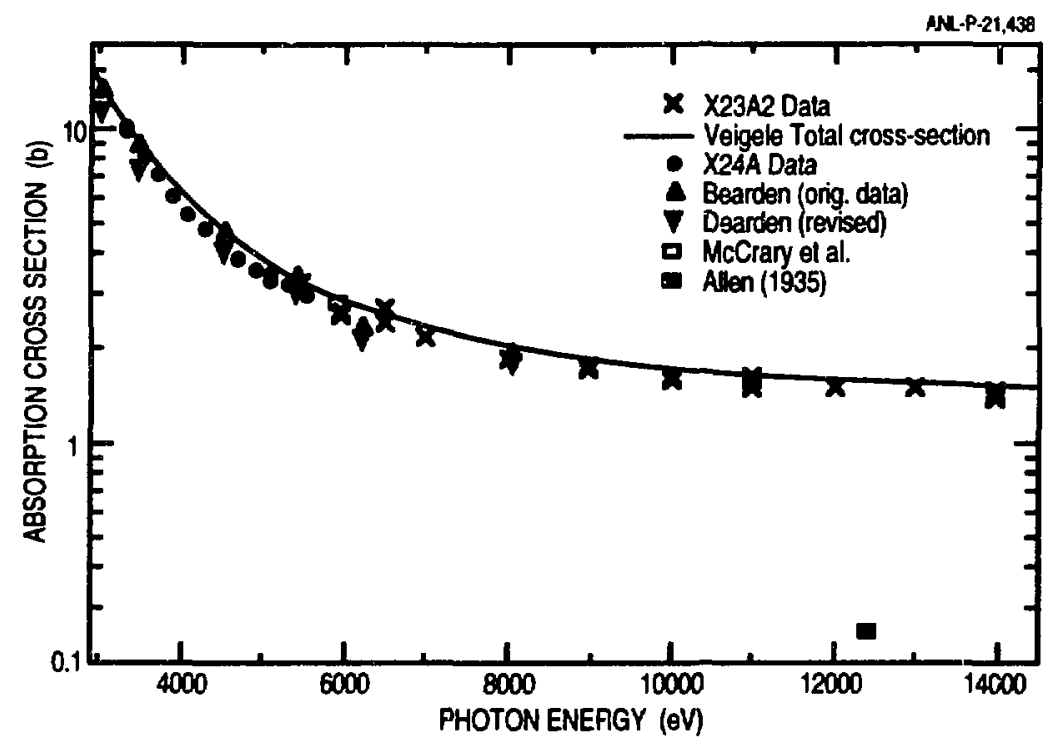

Fig. V-10. The iotal attenuation cross section for helium as a function of photon energy. Our measurements taken at the Brookhaven National Synchrotron Light Source are circles (•), taken at the X24A beam line, and crosses $(x)$, taken at the X23A2 beam line. Other measurements are also indicated.

\section{d. Absolute Photoabsorption Cross Section in Argon near the $\mathbf{K}$ Edge (Y. Azuma, H. G. Berry, P. L. Cowan, D. S. Gemmell, J. Suleiman,* and T. LeBrun)}

We made careful measurements of the absolute attenuation cross section for X-rays around the argon $\mathrm{K}$ edge. We used the pressure variation technique as in the helium attenuation measurements described above. The goal is to obtain a value for this cross section just above the $\mathrm{K}$ edge which is accurate to $1 \%$, in order to distinguish between several different calculations. Previous measurements, also made at the X24A beam line, are accurate to about $5 \%$. Data analysis is in progress.

\footnotetext{
*University of Illinois.
} 
e. Time-of-Flight Measurements of Ion Production Rates from Photoionization of Potassium and Argon near the K Edge (Y. Azuma, H. G. Berry, P. L. Cowan, D. S. Gemmell, and T. LeBrun)

We measured the photoion production from potassium around the $\mathrm{K}$ edge at $3.6 \mathrm{keV}$. The potassium source was a heated oven jet, with ions detected at a channel-plate detector at the end of a time-of-flight apparatus. Ions of charge states 2 through 8 were detected, and their relative intensities measured as a function of energy in the energy range -20 to $+20 \mathrm{eV}$ around the $\mathrm{K}$ edge.

Charge-state production rates in the photoionization of argon near the $\mathrm{K}$ edge are smooth and slowly varying. Although the $4 \mathrm{p}$ resonance has a width of less than $1 \mathrm{eV}$ in photoabsorption studies, the ion charge states $+4,+5$ and +6 , especially, have broader distributions of up to $10 \mathrm{eV}$. This extended variation below threshold is due to interferences in the photoionization from doublyexcited states of the argon neutral atom. We observed changes in the charge-state production to more than $50 \mathrm{eV}$ below threshold. The data analysis and comparison with theory is continuing.
f.
X-Ray Resonant Raman Spectroscopy (XRRS)
(P. L. Cowan, T. LeBrun,
R. D. Deslattes, ${ }^{*}$ and S. H. Southworth*)

$\mathrm{X}$-ray resonant Raman scattering has great promise as a high-resolution $\mathrm{X}$-ray probe of the electronic structure of matter. Unlike oiher spectroscopies the technique avoids the loss of energy resolution through inner-shell-hole-lifetime broad -ning. In addition, measurements of polarization and angular anisotropic effects are helpful in studies of symmetries of electronic states of atoms and molecules.

Work continues on studies of the $\mathrm{L}$ edge of xenon, where the lifetime broadening is a major feature of spectra previously observed. The many fluorescence channels enable us to study several possible polarization effects. Some molecular and solid-state tests are also in the planning stages.

g. Auger Resonant Raman Spectroscopy in Argon (T. LeBrun, Y. Azuma, S. H. Southworth, ${ }^{*}$ and M. MacDonald*)

We extended the resonant Raman techniques to electron spectroscopy to study the KLL Auger spectrum in experiments at the NSLS X24A beam line. The Raman spectrum shows excitation to the $4 p, 5 p$ and higher Rydberg resonances, while other Auger lines have negligible intensity. Their wider spacing in the ion spectrum yields much better resolution in the spectra, so that the higher Rydberg states can be partially resolved. Further measurements of this type will be helpful in identifying some of the structures of photoexcited states.

\section{h. Satellite Structure in the Argon 1s Photoelectron Spectrum (T. LeBrun, Y. Azuma, and S. H Southworth*)}

Atomic inner-sheil photoelectron spectra typically show several weak "satellite peaks" at higher ionization energy than the primary peak. These satellites are associated with final-state configurations corresponding to ionization of an inner-shell electron and excitation or ionization of one or more valence electrons. They indicate that the independent-electron picture is inadequate to describe the atomic structure in the photoionization process. We began a study of the photoelectron spectra of the suprathreshold region of the argon K region, using the NSLS X24A beam line. These measurements parallel our absorption spectra in this same region and can provide direct corroboration of our identifications. The work is in progress.

*National Institute of Standards \& Technology 
i. Planning for the Basic Energy Sciences Radiation Center (BESSRC) and other Facilities at the Advanced Photon Source (P. L. Cowan, P. Montano,* and G. Knapp*)

The detailed conceptual design for the BESSRC beam lines and experimental stations was accepted by the APS. Further details for these four beam lines are being developed. The atomic physics experimental station is the central station on the undulator beam line. The revisions to this undulator, now called the P-undulator, were approved. They will allow us to access photons over a continuous energy range between at least 4 and $35 \mathrm{keV}$, and hence provide a natural continuation to our experiments in the lower part of this range at the NSLS.

Progress continues on design and construction of high-performance X-ray mirrors, crystal monochromators and timing shutters. These devices have to maintain their alignment under the high radiated power loads expected at the APS.

*Material Sciences Division, ANL

\section{E. ACCELERATOR FACILITIES FOR ATOMIC PHYSICS}

The atomic physics group is involved in experiments at three Physics Division accelerator facilities - the ATLAS accelerator, BLASE (Beam-Laser), and the Dynamitron. The last two accelerators, BLASE and the Dynamitron, are dedicated solely to our atomic physics program.

At ATLAS one beam line is set up for atomic physics experiments. In addition, synchrotron-based experiments take place at the National Synchrotron Light Source (NSLS) X24A beam line. P. L. Cowan of our group is responsible for this beam line under the terms of an agreement with NIST (R. D. Deslattes).

A technical support group is available to assist with atomic physics experiments. The staff of this group includes: B. J. Zabransky, a Mechanical Engineer who is in charge of design, technical improvements, and the setup of experimental beam lines; A. E. Ruthenberg, operator of the Dynamitron, who works on technical projects at each of the other accelerators; C. A. Kurtz whose primary responsibility is BLASE and the synchrotron-based program.

The technical support group was largely responsible for implementing many new safety and environmental procedures for operation of all the Physics Division accelerators. Documentation for the procedures was rewritten and brought up to date.

\section{a. Operations at ATLAS (R. W. Dunford, B. J. Zabransky, and A. E. Ruthenberg)}

The atomic physics beam line set up at the ATLAS heavy-ion accelerator is used for a variety of experiments. The beam line has two focussing regions separated by ten meters. In the first region, an experimental setup used to study forbidden transitions consists of a chamber containing an array of four silicon X-ray detectors and a movable foil target. An optical encoder on the target translation stage determines precisely the position of the foil target. This apparatus has been used for studies of hyperfine quenching of forbidden decays. A target chamber located at the second focus on the beam line is used with a grazing-incidence monochromator for precision spectroscopy of highly-charged few-electron ions. A third region is being used to study interactions of ATLAS beams with vapor targets.

The split-pole magnetic spectrograph is also used by the atomic physics group to study ion-atom collisions. The target chamber and detectors required for these experiments are installed immediately before the runs and removed afterwards. Some upgrade of this beam line is in progress to allow its more efficient use for atomic experiments. 
b. BLASE Laboratory (L. Young and C. A. Kurtz)

BLASE is a laboratory designed for resonant interactions between a positive-ion beam and a laser. An ion source resides on a highly stabilized $150-\mathrm{kV}$ platform; mass analysis is provided by a $90^{\circ}$ magnet. Resonant radiofrequency transitions are induced by a speciaily designed if interaction region. The facility is dedicated primarily to high-precision spectroscopic experiments.

Substantial efforts have been directed toward complying with electrical, optical, and accelerator safety standards.

\section{c. Operation of the Dynamitron Facility (E. P. Kanter, A. E. Ruthenberg, B. J. Zabransky)}

The Dynamitron is a high-current stabilized 5-MV accelerator that can provide singly-charged beams of most atomic ions and many molecular ions. During this past year, the only use of the accelerator has been by the atomic physics program for work with fast molecular ions.

We previously installed in the Dynamitron high-voltage terminal a supersonic expansion source of vibrationally cold molecular ions. This source, which consists of a pulsed-jet supersonic gas expansion crossed by a beam of ionizing electrons, presented a majoì technological challenge because of the requirements of pumping background gas from the high-voltage terminal in order to achieve the high stagnation pressures necessary for vibrational cooling. This source has demonstrated substantial cooling of several molecular ions and we obtain beams with nearly pure ground-state populations.

The Dynamitron was scheduled to run experiments for 7 weeks in the summers of 1993 and 1994. When the machine was not being used for experiments, the technical staff assisted in the construction of beam lines, small accelerators, ion sources and accomplished machine modifications, repairs, and safety improvements. 


\section{OTHER EDUCATIONAL ACTIVITIES IN THE PHYSICS DIVISION}

The education of the next generation of scientists is an important component of the Division's programs. Many aspects of this activity are described in this report in connection with the speifific programs, most of which have an educational component involving university personnel. In addition, university user activities at ATLAS are listed separately, and lists of postdocs, and of graduate and undergraduate students involved in various activities are given. There are two additional programs related to education which are of importance to the Division but do not fit in the above categories: one is concerned with enhancing minority involvement in science, in particular in physics; the other involves scientific support for exhibits and outreach programs in connection with the SciTech Museum in nearby Aurora. These activities are described below.

\section{a. Enhancement of Minority Involvement in DOE Nuclear Physics Programs (B. Zeidman)}

During the past few years, there have been concentrated efforts to interact with a large number of minority students. These efforts have succeeded in attracting many qualified students to apply for participation in the programs of the Physics Division and other ANL divisions. Efforts are directed toward the identification of institutions with relatively strong physics programs and with faculty interested in stimulating their students to pursue research activities and summer programs. During visits to colleges, lectures are presented and are followed by discussion of activities in physics, at Argonne and other national laboratories, and the possibilities for graduate study, employment, etc. Additional activities included attending meetings of the Society for the Advancement of Chicanos and Native Americans in Science and of the Society of Black Physics Students. As a result of these efforts, 43 applications were received for the summer program in 1993. A total of 20 offers were made for the Summer Research Participation program in conjunction with Argonne's Department of Educational Programs. Additional institutions will be visited during this year and several meetings of minority groups will be attended. These ongoing interactions are generating institutional relationships that will enrich the physics programs in minority institutions and substantially eniiance minority involvement not only in nuclear physics, but in other branches of physics and science.

\section{b. Scientific Support of SciTech Museum Exhibits and Outreach Programs (M. Peshkin)}

SciTech (Science and Technology Interactive Center) is a small hands-on science museum located in Aurora, Illinois, not far from Argonne National Laboratory. Its constituency includes prosperous suburbs and economically disadvantaged minority communities in Aurora and Chicago. Its mission is to contribute to the country's scientific literacy initiative by offering handson experiences on the museum floor and through outreach programs eittended to school children, their teachers, and other groups.

Argonne's participation is focussed mainly on the development of exhibits to make the ideas of modern science and technology available to the public. This is an area in which traditional museums are weak, but in which SciTech has become a nationally recognized leader with the assistance of Argonne, Fermilab, nearby technological companies, and many volunteer scientists and engineers. We also participate in development and improvement of the museum's general exhibits and outreach programs. Argonne's Director Alan Schriesheim serves as a member of the museum's Board of Directors. Murray Peshkin serves part-time as the museum's Senior Scientist. Dale Henderson serves part-time as an exhibit developer. That work is supported by the Laboratory Directors discretionary funds. In addition, several members of the Physics Division voluntarily assist with exhibit development and the Division makes facilities available for that effort. 


\section{c. Exhibit Development (D. Henderson and M. Peshkin)}

"Building Blocks of the Universe", a major exhibition developed under a grant from the National Science Foundation, was completed in 1993 and is now operational at SciTech and at Ohio's Center for Science and Industry, in Columbus. The goal of the exhibit is to take the visitor in understandable steps from the macroscopic world to the levels of the atom, the nucleus, and the quark. Negotiations are underway to provide copies of some components of the exhibition at cost to other museums. The Argonne contribution to that project consisted primarily of writing some of the explanatory materials.

An exhibition called " $\mathrm{E}=\mathrm{mc}^{2 \mathrm{n}}$ is being developed under a grant from the Department of Energy. Its goal is to demystify Einstein's famous formula by demonstrating the conversion of matter to gamma rays and vice versa. The exhibition has two parts: 1) Positrons from a ${ }^{22 \mathrm{Na}}$ source annihilate with ambient electrons. The two resultant gamma rays are detected and their energies are confirmed. The visitor controls the process by moving absorbers. 2) Gamma rays from a Th source enter a diffusion cloud chamber and pair tracks are observed. The visitor may manipulate magnets and radioactive sources of positrons and of electrons. A prototype of the annihilation component has been completed and work is progressing on the final version. Work on the cloud chamber is still at an early stage but should be completed in 1994. Argonne people are playing a major role in both the conceptual design and the practical implementation of this segment.

An exhibition on quantum mechanics is still in the conceptual design stage. This project, which has been undertaken in collaboration with high school teachers, will primarily develop pedagogic experiments to be used in high schools. The Argonne contribution is primarily advisory at this stage. 


\section{STAFF MEMBERS OF THE PHYSICS DIVISION}

Listed below are the staff of the Physics Division for the year ending March 31, 1994.

The program headings indicate only the individual's current primary activity.

\section{EXPERIMENTAL NUCLEAR PHYSICS STAFF}

\section{Experimental Staff}

Irshad Ahmad, Ph.D., University of California, 1966

* Birger B. Back, Ph.D., University of Copenhagen, 1974

R. Russell Betts, Ph.D., University of Pennsylvania, 1972

Michael P. Carpenter, Ph.D., University of Tennessee, 1987

Cary N. Davids, Ph.D. California Institute of Technology, 1967

$\dagger$ Stuart J. Freedman, Ph.D., University of California, 1972

Donald F. Geesaman, Ph.D., State University of N.Y., Stony Brook, 1976

¥ Bruce G. Glagola, Ph.D., University of Maryland, 1978

$\S$ Walter F. Henning, Ph.D., Technical University of Munich, 1968

Roy J. Holt, Ph.D., Yale University, 1972

Harold E. Jackson, Jr., Ph.D., Cornell University, 1959

Robert V.F. Janssens, Ph.D. Univ. Catholique de Louvain, Belgium, 1978

I Cathleen Jones, Ph.D., California Institute of Technology, 1991

Sheldon B. Kaufman, Ph.D., University of Chicago, 1953

Teng Lek Khoo, Ph.D., McMaster University, 1972

I Walter Kutschera, Ph.D., University of Graz, Austria, 1965

** Christopher J. Lister, Ph.D., University of Liverpool, 1977

†† Jerry A. Nolen, Jr., Ph.D., Princeton University, 1965

$\ddagger \ddagger$ Richard C. Pardo, Ph.D., University of Texas, 1976

Karl Ernst Rehm, Ph.D., Technical University, Munich, 1973

$\S \S$ John P. Schiffer, Ph.D., Yale University, 1954

Kenneth W. Shepard, Ph.D., Stanford University, 1970

Kenneth Teh, Ph.D., Vanderbilt University, 1988

Alan H. Wuosmaa, Ph.D., University of Pennsylvania, 1989

Benjamin Zeidman, Ph.D., Washington University, 1957

* On assignment at SUNY until August 1993.

$\dagger$ On leave at the University of California, Berkeley until October 1, 1994.

‡ ATLAS User Program Administrator.

$\S$ Director of the Physics Division.

I Postdoctoral Appointee until July 1993.

On leave of absence at the University of Vienna until August 1994.

** Joined the Physics Division in January $19: 4$.

$\dagger \dagger$ Director of the ATLAS Facility.

$\ddagger$ ATLAS Operations Manager

$\S \S$ Associate Director of the Physics Division. Joint appointment with the University of Chicago. 
Experimental Staff/Special Appointments

* Lowell M. Bollinger, Ph.D., Cornell University, 1951

* Melvin S. Freedman, Ph.D., University of Chicago. 1942

* Alexander Langsdorf, Jr., Ph.D., Massachusetts Inst. oî Techrology, 1937

* Michael Paul, Ph.D., Hebrew University of Jerusalem, 1973

* G. Roy Ringo, Ph.D., University of Chicago, 1940

* George E. Thomas, B.A., Illinois Wesleyan, 1943

* Ian L. Yntema, Ph.D., Free University of Amsterdam, 1952

\section{THEORETICAL NUCLEAR PHYSICS STAFF}

\section{Theoretical Physics Staff}

Richard R. Chasman, Ph.D., University of California, 1959

Fritz Coester, Ph.D., University of Zurich, 1944

Henning Esbensen, Ph.D., University of Aarhus, 1977

Stephen Landowne, Ph.D., Carnegie-Mellon University, 1970

Tsung-Shung Harry Lee, Ph.D., University of Pittsburgr 1973

Steven C. Pieper, Ph.D., University of Illinois, 1970

Craig T. Roberts, Ph.D., Flinders University of South Australia, 1989

Robert B. Wiringa, Ph.D., University of Illinois, 1978

Theoretical Staff/Special Appointments

$\dagger$ Arnold R. Bodmer, Ph.D., Manchester University, 1953

* Lloyd Hyman, Ph.D., Massachusetts Institute of Technology, 1959

* Dieter Kurath, Ph.D., University of Chicage. 1951

* Harry J. Lipkin, Ph.D., Princeton University, 1950

† James E. Monahan, Ph.D., St. Louis University, 1951

$\S$ Vijay Pandharipande, Ph.D., University of Bombay, 1969

* Murray Peshkin, Ph.D., Cornell University, 1951

* Post-retirement research participant.

$\dagger$ Post-retirement research participant from the University of Illinois, Chicago

$\mp$ Post-retirement research participant. Deceased September 5, 1993.

$\S$ Research participant from the University of Illinois, Urbana. 


\section{ATOMIC AND MOLECULAR PHYSICS}

\section{A omic and Molecuiar Physics Staff}

* Yoshiro Azuma, Ph.D., University of Oregon, 1985

H. Gordon Berry, Ph.D., University of Wisconsin, 1967

Paul L. Cowan, Ph.D., Pennsylvania State University, 1977

Robert Dunford, Ph.D., University of Michigan, 1978

Donald S. Gemmell, Ph.D., Australian National University, 1960

Elliot P. Kanter, Ph.D., Rutgers University, 1977

$\dagger$ Zeev Vager, Ph.D. Weizmann Institute of Science, 1962

¥ Linda Young, Ph.D., University of California, Berkeley, 1981

Atomic And Molecular Physics Staff/Special Appointments

$\S$ William J. Childs, Ph.D., University of Michigan, 1956

$\S$ F. Paul Mooring, Ph.D., University of Wisconsin, 1951

$\S$ Gilbert J. Perlow, Ph.D., University of Chicago, 1940

\section{TEMPORARY APPOINTMENTS}

\section{TERM APPOINTMENTS}

Torben Lauritsen, Ph.D., State University of New York, 1990

David H. Potterveld, Ph.D., Caltech, 1988

\section{POSTDOCTORAL APPOINTEES}

Rami Ali (from Kansas State University, Manha :AS, Kansas): Atomic physics at ATLAS. (August 1993--

Daniel Blumenthal (University of Pennsylvania, Philadelphia, Pennsylvania): Heavy-ion research at ATLAS. (January 1994-- )

Song Cheng (from Kansas State University, Manhattan, Kansas): Atomic physics at ATLAS. (August 1991--August 1993)

* Terminated December 1993.

$\div$ Joint Appointment with Weizmann Institute of Science, Rehovot, Israel.

$\ddagger$ On sabbatical at JILA until September 1993.

$\S$ Post-retirement research participant. 
Postdoctoral Appointees (cont'd)

Benjamin F. Crowell (from Yale University, New Haven, Connecticut): Heavy-ion research at ATLAS.

(June 1993--

Martin Freer (from Birmingham University, Birmingham, UK):

Heavy-ion physics at ATLAS.

(April 1991--April 1993)

Richard Harkewicz (from Michigan State University, E. Lansing, Michigan):

Accelerator development and research at ATLAS.

(March 1992--

Roland Henry (from Rutgers University, New Brunswick, New Jersey):

Heavy-ion physics at ATLAS.

(January 1992-- )

* Kihun Joh (from Michigan State University, E. Lansing, Michigan):

Research at ATLAS.

(October 1993--December 1993)

$\dagger$ Cathleen Jones (from California Institute of Technology, Pasadena, California): Medium-energy physics research. (October 1992--July 1993)

Thomas LeBrun (from University of Paris, France):

Atomic physics using synchrotron light sources.

(November 1991-- )

Vassilious Pavassiliou (from Yale University, New Haven, Connecticut):

Medium-energy physics research.

(May 1991-r )

Heikki Penttila (from University of Jyväskylä, Finland):

Research with the FMA at ATLAS

(Octoher 1992-- )

Bernard Matthew Poelker (from Northwestern University, Evanston Illinois):

Medium-energy physics research.

(January 1992--February 1994)

Martin Rhein (from Institut fur Kernphysik, Darmstadt, Germany):

Heavy-ion physics at ATLAS.

(January 1993-- )

* Resident Graduate Student until October 1993.

$\dagger$ Joined the regular staff in July 1993. 


\section{TECHNICAL AND ENGINEERING STAFF \\ (and areas of activity)}

Kevin G. Bailey (B.S. University of Nebraska, 1989). SOS construction for CEBAF.

Brian T. Batzka,(B.S. University of Houston, 1992). ATLAS operator.

Peter J. Billquist

ECR heavy-ion source, ATLAS operation.

John M. Bogaty (A.A.S. DeVry, 1961).

Electric l systems, ATLAS operation and development.

Benny G. Clifft (A.S.E.E., DeVry, 1959).

Electrical systems, ATLAS operation and development.

Joseph Falout (B.S.M.E. University of Illinois, 1970).

Experimental equipment design.

John P. Greene (M.S. DePaul University, 1982).

Target preparation.

Ray E. Harden (A.A.S. Milwaukee School of Engineering, 1957). ATLAS operator

Dale J. Henderson (B.S. Elmhurst College, 1951).

Detector development, technical assistance, heavy-ion physics.

James M. Joswick (A.A.S. Milwaukee School of Engineering, 1964).

ATLAS experimental equipment maintenance, technical assistance, heavy-ion physics.

Raymond B. Kickert

ATLAS experimental equipment maintenance, technical assistance, heavy-ion physics.

Robert Kowalczyk (M.S. Northeastern Illinois University, 1983).

Technical assistance, medium-energy physics.

Charles A. Kurtz (M.S. University of Arkansas, 1984).

Technical assistance, atomic physics.

* Paul Markovich (B.S. Purdue University, 1972).

Surface chemistry, ATLAS development and operation.

Thomas P. Mullen (B.S. Marquette University, 1966).

Division ESH/QA engineer.

Floyd Munson, Jr. (A.A.S. DeVry, 1966).

Control system for ATLAS.

Kirt Nakagawa (B.S. University of Illinois, 1988).

ATLAS operator.

*Post-retirement appointee. 
Technical and Engineering Staff (cont'd)

Bruce G. Nardi (A.A.S. Morton Jr. College, 1967; A.A.S. DeVry, 1969). Electronics design and maintenance.

James R. Specht (A.A.S. DeVry, 1964).

Cryogenics engineer. ATLAS development and operation.

Philip Strickhorn (B.S. DeVry, 1990).

Electrical and technical assistance with ATLAS operations.

Anne Sutherland (B.S. University of Rochester, 1993).

Technical assistance with the ATLAS cryogenic system.

Brian J. Tieman (B. A. North Central College, 1992). ATLAS operator.

Ian R. Tilbrook (B.S. Pennsylvania State University, 1987).

ATLAS operator.

Richard Vondrasek (B.S. University of Illinois, 1990).

ATLAS operator.

Gregory Wiemerslage (B.S. Elmhurst College, 1990).

Technical assistance with ATLAS operations.

Philip R. Wilt (Johnstown Technical School 1973).

Electronics design and maintenance.

* Bruce J. Zabransky (M.S. University of Illinois, Chicago, 1973).

Dynamitron operation.

Anthony R. Zeuli (B.A. Hamline University, 1990).

Technical assistance. Weak interactions physics.

Gary P. Zinkann (B.S. DeVry, 1975).

Superconducting resonators and linac maintenance, for ATLAS operations.

\section{ADMINISTRATIVE STAFF}

$\dagger$ Allan Bernstein, M.B.A., Rosary College, 1986

$\ddagger$ James E. Nelson, B.A., University of Illinois, 1975

\footnotetext{
* In charge of Dynamitron operation.

$\dagger$ Assistant Director of the Physics Division.

$\ddagger$ Manager, Division Operations.
} 


\section{VISITORS AND STUDENTS}

LONG-TERM VISITORS (at Argonne more than 4 months)

Nemitala Added (University of Sao Paulo, Brazil):

Linac development.

(March 1991--March 1993)

* Miron Amusia (Ioffe Institute, St. Petersburg, Russia):

Atomic physics research.

(May 1993--

Daniel Ashery (Tel Aviv University, Jerusalem, Israel):

Medium-energy physics research.

(July 1992--May 1993)

Thomas Dossing (University of Copenhagen):

Heavy-ion research.

(September 1993-- )

Marcello D. Farraretto (University of Sao Paulo, Brazil):

ATLAS development.

(April--July 1993; September--October 1993)

David Gassamann (Technical University of Munich, Germany):

Heavy-ion research at ATLAS.

(January 1994-- )

$\dagger$ Timothy J. Graber (University of Illinois-Chicago):

Accelerator-based atomic physics.

(June--October 1993)

Robert Hermann (Technical University of Munich, Germany):

Heavy-ion research at ATLAS.

(June 1993--

Cheng-Lie Jiang (China Institute of Atomic Energy, Beijing, China):

Heavy-ion research at ATLAS.

(October 1992-- )

Ajith Kumar (Nuclear Science Centre, New Delhi, India):

ATLAS development.

(January 1994--

Prakash Potukuchi (Nuclear Science Center, New Delhi, India):

ATLAS development.

(October 1991--

$$
\text { ) }
$$

* 1993-94 Argonne Fellow.

$\dagger$ Resident Graduate Student until June 1993. 
Amit Roy (Nuclear Science Center, New Delhi, India):

ATLAS development.

(January 1994--

Norbert Schmidt (Fachhochschule, Munich, Germany):

Heavy-ion research at ATLAS.

(March 1994-- )

* Carol Tanner (University of Notre Dame, Indiana):

Atomic physics at BLASE.

(Näch 1992-- )

$\dagger$ Guangsheng Xu (Yale University, New Faven, Connecticut):

Heavy-ion research at ATLAS.

(January 1994-- )

SHORT-TERM VISITORS (at ANL less than 4 months)

$\ddagger$ Karen Bardeen (Oak Park/River Forest High School, Oak Park, Illinois): Accelerator-based atomic physics. (June 1993--August 1993)

Axel Bender (University of Tubingen, Germany):

Nuclear theory studies.

(July--August 1993)

Rudolph E. Ganz (University of Heidelberg):

APEX experiment at ATLAS.

(July--September 1993)

$\S$ Edward Hohman (York Township High School, Lyons, Illinois):

Summer student coordinator.

(June--August 1993)

Paul Helmut Mokler (GSI, Darmstadt, Germany):

Atomic physics at ATLAS.

(February --March 1994)

Satoshi Nozawa (Josai University, Saitama, Japan):

Nuclear theory studies.

(August 1993)

Stanislav Popov (Institute for Nuclear Physics, Novositirsk, Russia):

Medium-energy physics studies.

(May 1993)

† Akunuri Ramayya (Vanderbiilt University, Nashville, Tennessee):

Heavy-ion research at ATLAS.

(June--August 1993)

* Guest Faculty Research Participant.

$\dagger$ Faculty Research Participant.

$\ddagger$ Department of Energy Teacher Research Associate Program.

$\S$ Guest Faculty Research Participant. Summer student coordinator. 
Amit Roy (Nuclear Science Centre, New Delhi, India):

ATLAS development.

(January 1994--

Toru Sato (Osaka University, Japan):

Nuclear theory studies.

(July--August 1993)

Andrey Sukhanov (Institute for Nuclear Physics, Novosibirsk, Russia):

Medium-energy physics studies.

(October 1993)

Dmitri Toporkov (Institute for Nuclear Physics, Novosibirsk, Russia):

Medium-energy physics.

(May--June 1993)

Daniel Zajfman (Weizmann Institute of Science, Rehovoth, Israel): Accelerator-based atomic physics.

(August 1993)

\section{RESIDENT GRADUATE STUDENTS}

Ian Bearden (Purdue University, W. Lafayette, Indiana):

Heavy-ion research at ATLAS.

(January 1990--October 1993)

Kevin Beyer (Michigan State University, E. Lansing, Michigan):

Heavy-ion research at ATLAS.

(August 1992--

Larry Brown (Vanderbilt University, Nashville, Tennessee):

FMA development at ATLAS

(January 1994-- )

Kanwarjit S. Bindra (Vanderbilt University, Nashville, Tennessee):

FMA development at ATLAS.

(May 1990--

Kin Chi Chan (Yale University, New Haven, Connecticut):

APEX experiment at ATLAS.

(June 1992--

John C. Gehring (University of Chicago, Chicago, Illiniois):

Heavy-ion research at ATLAS.

(June 1990--

Timothy J. Graber (University of Illinois, Chicago):

Coulomb-explosion studies.

(October 1989--June 1993)

Kihun Joh (Michigan State University):

ATLAS development.

(June 1992-- October 1993) 
Nicholas Kaloskamis (Yale University, New Haven, Connecticut): APEX experiment at ATLAS.

(November 1991-- )

Kris Kukla (University of Notre Dame):

Atomic physics research.

(July 1992-- August 1993)

Manquing Liu (Queen's University, Kingston, Ontario, Canada): APEX experiment at ATLAS.

(September 1992-- )

David Nisius (Purdue Univ ersity, W. Lafayette, Indiana): Heavy-ion research at ATLAS.

(June 1993 --

Aloy Perera (University of Rochester, Rochester, New York): APEX experiment at ATLAS. (August 1993 --

Michael Pichowsky (University of Pittsburgh, Pennsylvania): Theoretical physics studies.

(September 1993-- )

Laura Richter (Purdue University, W. Lafayette, Indiana): Heavy-ion research at ATLAS. (May--August 1993)

Dante Roa (Florida State University):

Research with APEX at ATLAS.

(May 1992--

Lesley Smith (University of Kansas, Manhattan, Kansas):

Theoretical physics studies.

(August 1993--December 1993)

Jamal Suleiman (University of Illinois, Chicago, Illinois):

Atomic physics research.

(October 1991-- )

Mark Wolanski (University of Chicago, Chicago, Illinois):

Weak interaction studies.

(July 1991--

\section{Guest Graduate Students}

Mohammad Talebian Darzi (University of Illinois, Chicago, Illinois):

Atomic physics research.

(January 1993--

Aloy Perera (University of Rochester, Rochester, New York):

APEX experiment at ATLAS.

(August 1991--August 1993) 
Robert Rafac (University of Notre Dame, Notre Dame, Indiana): Atomic physics research.

(March 1992-- )

Zhou Yu (Northwestern Unviersity, Evanston, Illinois):

Intermediate-energy physics.

(September 1987-- )

\section{UNDERGRADUATE STUDENTS}

Erik Alldredge (Worcester Polytechnic Institute)

Gordon Annan (Dillard University)

Helene Borden (State University of New York-Geneseo)

Jonathan Brumley (Rice University)

Donna Chess (North Central College)

Diane Eschliman Nebraska Wesleyan University)

Melanie Felton (Andrews University)

Anne Flewelling (College of Wooster, Ohio)

David Flory (University of Michigan-Flint)

Mark Gaskin (Xavier University)

Jennifer Gerbi (Bard College)

Gregory Gruel (Lincoln University)

Steven Harfenist (Buffalo State College)

Taliver Heath (Florida State University)

Jay Kreibich (University of Illiinois-Urbana)

Hong Meng (Rensselaer Polytechnic Institute)

Hassan Moore (Dillard University)

Richard Pelaia (Florida Institute of Technology)

Christian Roehrig (North Central College)

Brian Rutherford (College of St. Francis)

Michael Schueller (Youngstown State University)

Joseph Stacy (Norfolk State University)

Christopher Stepanek (College of St. Francis)

David Sowinski (Lewis University)

James Thompson (Florida State University)

Dale Visser (Western Michigan University)

Gregory Voth (Wheaton College)

Kimberly Weeks (Moraine Valley Community College)

William Weintraub (Francis Marion University)

Brian Williams (Norfolk State University)

James Wisniewski (Purdue University)

Matthew Wolf (Miami University of Ohio)

PRE-COLLEGE PROGRAM (Just Graduated from High School) (June--August 1993)

Andrea Culumber (Glenbard West High School)

Farahnaz Moazezi (Oak Park/River Forest High School)

Heidi Reichenbach (Plainfield High School)

Teresa Soledad (Mt. Assisi High School)

Chen Tan (Oak Park/River Forest High School) 


\section{PUBLICATIONS FROM APRIL 1, 1993 THROUGH MARCH 31, 1994}

(The arrangement follows approximately the Table of Contents)

\section{HEAVY-ION RESEARCH}

Octupole Shapes in Nuclei

I. Ahmad and P. A. Butler

Ann. Rev. Nucl. and Part. Sci. 43 , 71-116 (1993)

Accelerator Mass Spectrometry of ${ }^{59} \mathrm{Ni}$ in Extraterrestrial Matter

W. Kutschera, I. Ahmad, B. G. Glagola, R. C. Pardo, K. E. Rchm, D. Berkovits, M. Paul, J. R. Arnold, and K. Nishiizumi

Nucl. Instrum. Methods $\underline{73}$, 403-412 (1993)

A Fast Low Noise Silicon Detector for Electron Spectroscopy up to $1 \mathrm{MeV}$

L. Evensen, A. Hanneborg, T. Happ, A. Wuosmaa, and R. Betts

Nucl. Instrum. Methods A326, 136-143 (1993)

Collective and Quasiparticle Structures in ${ }^{192} \mathrm{~Pb}$

A. J. M. Plompen, M. N. Harakeh, W. H. A. Hesselink, G. van't Hof, N. Kalantar- Nayestanaki,

J. P. S. van Schagen, M. P. Carpenter, I. Ahmad, I. G. Bearden, R. V. F. Janssens, T. L. Khoo,

T. Lauritsen, Y. Liang, U. Garg, W. Reviol, and D. Ye

Nucl. Phys. A562, 61-87 (1993)

Non-Collective Degrees of Freedom in ${ }^{192} \mathrm{~Pb}$

A. J. M. Plompen, J. Blomqvist, M. P. Carpenter, M. N. Harakeh, W. A. H. Hesselink,

R. V. F. Janssens, R. J. Liotta, and N. Sandalescu

Nucl. Phys. A562, 88-100 (1993)

Accelerator Mass Spectrometry: Counting Atoms Rather Than Decays

W. Kutschera

Nuci. Phys. News $\underline{3}, 15-21$ (1993)

Sub-Barrier Fusion Cross Sections in $58,64 \mathrm{Ni}+{ }^{92,100} \mathrm{Mo}$ Studied with the Gas-filled Magnet Technique

K. E. Rehm, H. Esbensen, J. Gehring, B. Glagola, D. Henderson, W. Kutschera, M. Paul,

F. Soramel, and A. H. Wuosmaa

Phys. Lett. B $\underline{317}$, 31-35 (1993)

Identification of ${ }^{183} \mathrm{Hg}$. Identical Bands in $183,185 \mathrm{Hg}$

K. S. Bindra, A. V. Ramayya, W. C. Ma, B. R. S. Babu, J. H. Hamilton, L. Chaturvedi,

J. Kormicki, R. V. F. Janssens, C. N. Davids, I. Ahmad, I. G. Bearden, M. P. Carpenter,

W. Chung, D. Henderson, R. G. Henry, T. L. Khoo, T. Lauritsen, Y. Liang, H. Penttilä,

F. Soramel, C. Baktash, W. Nazarewicz, and J. A. Sheikh

Phys. Lett. B $\underline{318}$, 41-46 (1993)

Electron-Nucleus Interactions in Few-Electron Fe Ions

W. R. Phillips, J. Copnell, D. W. Banes, K. E. Rehm, I. Ahmad, B. G. Glagola, W. Henning,

W. Kutschera, and J.P. Schiffer

Phys. Rev. A 4ㄱ, 3682-3691 (1993) 
Recoil-Free Absorption and Scattering of Light from Confined Crystalline Ionic Systems J. P. Schiffer

Phys. Rev. A 47, 5193-5195 (1993)

New $\mathrm{N}=84$ Isotone: ${ }^{136} \mathrm{Te}$

M. A. C. Hotchkis, J. L. Durell, J. Copnell, A. S. Mowbray, J. Fitzgerald, W. R. Phillips, I. Ahmad, M. P. Carpenter, R. V. F. Janssens, T. L. Khoo, E. F. Moore, L. R. Morss,

Ph. Benet, and D. Ye

Phys. Rev. C 47, 1294 (1993)

Yrast Isomers in Exotic $\mathrm{N}=81$ Nucleus $151 \mathrm{Yb}$ Studied Using the Argonne Fragment Mass Analyzer

D. Nisius, B. Fornal, I. G. Bearden, R. Broda, R. H. Mayer, Z. W. Grabowski, P. J. Daly, C. N. Davids, I. Ahmad, B. B. Back, K. Bindra, M. P. Carpenter, W. Chung, D. Henderson, R. G. Henry, R. V. F. Janssens, T. L. Khoo, T. Lauritsen, Y. Liang, F. Soramel, and A. V. Ramayya

Phys. Rev. C 47, 1929 (1993)

Energy Dependence of Fusion Evaporation-Residue Cross Sections in the ${ }^{28} \mathrm{Si}+{ }^{28} \mathrm{Si}$ Reaction

M. F. Vineyard, J. S. Bauer, C. H. Gosdin, R. S. Trotter, D. G. Kovar, C. Beck,

D. J. Henderson, R. V. F. Janssens, B. D. Wilkins, G. Rosner, P. Chowdhury, H. Ikezoe,

W. Kühn, J. J. Kolata, J. D. Hinnefeld, C. F. Maguire, J. F. Mateja, F. W. Prosser, and G. S. F. Stephans

Phys. Rev. C $\underline{47}, 2374$ (1993)

Lack of Evidence for a Superdeformed Band in ${ }^{192} \mathrm{~Pb}$

A. J. M. Plompen, M. N. Harakeh, W. H. A. Hesselink, G. van't Hof, N. Kalantar-Nayestanaki, J. P. S. van Schagen, R. V. F. Janssens, I. Ahmad, I. G. Bearden, M. P. Carpenter, T. L. Khoo, T. Lauritsen, Y. Liang, U. Garg, W. R viol, and D. Ye

Phys. Rev. C 47, 2378 (1993)

Neutron Transfer Reactions at Large Distances

K. E. Rehm, B. G. Glagola, W. Kutschera, F. L. H. Wolfs, and A. H. Wuosmaa

Phys. Rev. C 4 7, 2731-2739 (1993)

Collective Oblate Bands in $196 \mathrm{~Pb}$

J. R. Hughes, Y. Liang, R. V. F. Janssens, A. Kuhnert, J. A. Becker, I. Ahmad, I. G. Bearden,

M. J. Brinkman, J. Burde, M. P. Carpenter, J. A. Cizewski, P. J. Daly, M. A. Deleplanque,

R. M. Diamond, J. E. Draper, C. Duyar, B. Fornal, U. Garg, Z. W. Grabowski, E. A. Henry,

R. G. Henry, W. Hesselink, N. Kalantar- Nayestanaki, W. H. Kelly, T. L. Khoo, T. Lauritsen,

R. H. Mayer, D. Nissius, J. R. B. Cliveira, A. J. M. Plompen, W. Reviol, E. Rubel, F. Soramel, and F. S. Stephens,

Phys. Rev. C 47, R1337 (1993)

Mass Asymmetry Dependence of Scission Times in the Reactions of $18.5 \mathrm{~A} \mathrm{MeV}{ }^{136} \mathrm{Xe}+{ }^{48} \mathrm{Ti}$ M. Gui, K. Hagel, R. Wada, Y. Lou, D. Utley, B. Xiao, J. Li, J. B. Natowitz, G. Enders, W. Kuhn, V. Metag, R. Novotny, O. Schwalb, R. J. Charity, R. Freifelder, A. Gobbi, W. Henning, K. D. Hildenbrand, R. Mayer, R. S. Simon, J. P. Wessels, G. Casini, A. Olmi, and A. A. Stefanini

Phys. Rev. C $\underline{48}, 1791$ (1993) 
Spectroscopy of the Superdeformed Band in $196 \mathrm{~Pb}$

E. F. Moore, Y. Liang, R. V. F. Janssens, M. P. Carpenter, I. Ahmad, I. G. Bearden,

P. J. Daly, M. W. Drigert, B. Fornal, U. Garg, Z. W. Grabowski, H. L. Harrington,

R. G. Henry, T. L. Khoo, T. Lauritsen, R. H. Mayer, D. Nisius, W. Reviol, and M. Sferrazza Phys. Rev. C $\underline{48}, 2261$ (1993)

Spectroscopy of ${ }^{186} \mathrm{~Pb}$ with Mass Identification

A. M. Baxter, A. P. Byrne, G. D. Dracoulis, R. V. F. Janssens, I. G. Bearden, R. G. Henry,

D. Nisius, C. N. Davids, T. L. Khoo, T. Lauritsen, H. Penttilä, D. J. Henderson, and

M. P. Carpenter

Phys. Rev. C $\underline{48}$, R2140 (1993)

First Operational Experience with the Positive-Ion Injector of ATLAS

L. M. Bollinger, R. C. Pardo, K. W. Shepard, J. M. Bogaty, B. E. Clifft, F. H. Munson, and

G. Zinkann

Proceedings of the 6th International Conference on Electrostatic Accelerators and Associated

Boosters, Padova, Italy, 1-5 June 1992; Nucl. Instrum. Methods A328, 22 1-230 (1993)

Radioactive Target and Source Development at Argonne National Laboratory

J. P. Greene, I. Ahmad, and G. E. Thomas

Proceedings of the 16th World Conference on International Nuclear Target Development

Society, Padova, Italy, September 21-25, 1992; Nucl. Instrum. Methods A334, 101-110

(1993)

Target Thinning Using a Saddle Field Ion Source

G. E. Thomas and J. P. Greene

Proceedings of the 16th World Conference on International Nuclear Target Development

Society, Padova, Italy, September 21-25, 1992; Nucl. Instrum. Methods A334, 132-136 (1993)

Energy Compensation of an Isolde-Type Isotope Separator

C. N. Davids

Proceedil ss of the 12th International Conference on Electromagnetic Isotope Separators \&

Techniques Related to their Applications, Sendai, Japan, 2-6 September 1991; Nucl. Instrum.

Methods B70, 435-440 (1992)

The Positive-Ion Injector of ATLAS: Design and Operating Experience

L. M. Bollinger, R. C. Pardo, K. W. Shepard, P. J. Billquist, J. M. Bogaty, B. E. Clifft,

R. Harkewicz, F. H. Munson, J. A. Nolen and G. P. Zinkann

Proceedings of the Twelfth International Conference on the Application of Accelerators in

Research and Industry, Denton, Texas, November 2-5, 1992; Nucl. Instrum. Methods B79, 753-757 (1993)

New Results on Superdeformed Bands in $\mathrm{Hg}$ and $\mathrm{Tl}$ Nuclei

M. P. Carpenter, R. V. F. Janssens, Y. Liang, I. G. Bearden, 1. Ahmad, M. W. Drigert,

U. Garg, R. G. Henry, J. M. Lewis, T. L. Khoo, T. Lauritsen, S. Pilotte, W. Reviol,

L. L. Riedinger, F. Soramel, and C.-H. Yu

Proceedings of the International Symposium on Rapidly Rotating Nuclei 1992, Tokyo, Japan, 26-30 October 1992; Nucl. Phys. A557, 57c-66c (1993) 
Study of the Superdeformed Band in ${ }^{194} \mathrm{~Pb}$ and ${ }^{192} \mathrm{Hg}$ with EUROGAM

F. Hannachi, C. Schuck, G. Bastin, I. Deloncle, B. Gall, M. G. Porquet, A. G. Smith,

F. Azaiez, C. Bourgeois, J. Duprat, A. Korichi, N. Perrin, N. Poffe, H. Sergolle, A. Astier,

Y. Le Coz, M. Meyer, N. Dedon, M. Bentley, J. Simpson, J. F. Sharpey-Schafer, M. J. Joyce,

C. W. Beausang, P. Fallon, E. S. Paul, P. J. Dagnall, S. A. Forbes, S. Gale, P. M. Jones,

R. Wadsworth, R. M. Clark, M. M. Aleonard, D. Curien, G. De France, M. P. Carpenter, and

R. G. Henry

Proceedings of International Symposium on Rapidly Rotating Nuclei, October 26-30, 1992, Tokyo, Japan; Nucl. Phys. A557, 75c-82c (1993)

Spectroscopy of $96,97,98 \mathrm{Ru}$ : Possible Emergence of Colleciivity at $\mathrm{N} \geq 52$

W. Reviol, U. Gari, I. Ahmad, A. Aprahamian, M. P. Carpenter, B. F. Davis,

R. V. F. Janssens, T. L. Khoo, T. Jauritsen, Y. Liang, S. Naguleswaran, J. C. Walpe, and D. Ye

Proceedings of International Symposium on Rapidly Rotating Nuclei, Tokyo, Japan, October 26-30, 1992 ; Nucl. Phys. A557, 39lc-400c (1993)

Experiments Using the Argonne Fragment Mass Analyzer

C. N. Davids, B. Back, I. G. Bearden, K. Bindra, C. R. Bingham, R Broda, M. P. Carpenter, W. Chung, F. J. Daly, B. Fornal, Z. W. Grabowski, D. J. Henderson, R. G. Henry,

R. V. F. Janssens, T. L. Khoo, T. Lauritsen, Y. Liang, R. H. Mayer, D. M. Moltz, D. Nisius,

A. V. Ramayya, J. D. Robertson, F. Scarlassara, F. Soramel, P. Spolaore, K. S. Toth, and

W. B. Walters

Proceedings of the XVI Symposium on Nuclear Physics, Oaxtepec, MX, 5-8 January 1993, Revistas Mexicana de Fisica 39, Suplemento 2, pp. 56-63 (1993)

Partis:- Production in Nucleus-Nucleus Collisions at Near-Relativistic Energies

$\therefore$. Henning

Frontier Topics in Nuclear and Astrophysics - Graduate Lectures, IOP Publishing Ltd., London (1993), eds. Z. Sujkowski and G. Szeflinska, pp. 1-14

Distributions of Reaction Strengths and the Quest for a Unified Picture

W. Henning

International Symposium "Towards a Unified Picture of Nuclear Dynamics", Nikko, Japan, June 6-8, 1991; AIP Conference Proceedings 250, 515-526 (1992)

First Results from the Fragment Mass Analyzer at ATLAS

C. N. Davids, I. Ahmad, B. B. Back, I. G. Bearden, R. R. Betts, K. Bindra, C. R. Bingham,

D. J. Blumenthal, R. Broda, M. P. Carpenter, P. Chowdhury, W. Chung, B. Crowell,

P. J. Daly, B. Fornal, S. J. Freeman, M. Freer, J. Gehring, Z. W. Grabowski, D. J. Herderson,

R. Henry, R. V. F. Janssens, T. Lauritsen, Y. Liang, C. J. Lister, R. H. Mayer, D. M. Moltz,

D. Nisius, A. V. Ramayya, J. D. Robertson, F. Scarlassara, P. Spolaore, F. Soramel, and W. B. Walters

Proceedings of the 6th International Conference on Nuclei Far from Stability, and 9th International Conference on Atomic Masses and Fundamental Constants, Bernkastel-Kues, Germany, 26 July-1 August 1992; Inst. Phys. Conf. Ser. 132, Sect. 8, 931-93.3 (1993) 
Investigation of Proton-Rich Platinum and Mercury Isotopes with the Fragment Mass Analyzer at ATLAS and the Isotope Separator at UNISOR

K. S. Toth, C. N. Davids, Y. A. Akovali, B. B. Back, K. Bindra, C. R. Bingham, H. K. Carter,

W. Chung, Y. Hatsukawa, D. J. Henderson, T. Lauritsen, P. F. Mantica, D. M. Moltz,

A. V. Ramayya, J. D. Robertson, and W. B. Walters

Proceedings of the 6th International Conference on Nuclei Far from Stability, Bernkastel-

Kues, Germany, 19-24 July 1992, eds. R. Neugart and A. Wohr (IOP Publishing Ltd.,

Bristol, England), Institute of Physics Conference Series No. 132, ISBN 0-7503-0262-3, pp.

589-594 (1993)

Status and Developmental Efforts of the PIIECR Ion Source

R. Harkewicz, P. J. Billquist, and R. C. Pardo

Proceedirgs of the 11 th International Workshop on Electron Cyclotron Resonance Jon Sources (ECRIS 11), Groningen, The Netherlands, May 6-7, 1993, ed. A. G. Drentje, KVI-Report 996, p. 53-54 (1993)

\section{ATLAS DEVELOPMENT AND OPERATION}

First Operational Experience with the Positive-Ion Injector of ATLAS

L. M. Bollinger, R. C. Pardo, K. W. Shepard, J. M. Bogaty, B. E. Clifft, F. H.

Munson, and

G. Zinkann

Proceedings of the 6th International Conference on Electrostacic Acelerators and Associated Boosters, Padova, Italy, 1-5 June 1992; Nucl. Instrum. Methods A328, $221-230(1993)$

Some Aspects of Linacs as Applied to the ISL Benchmark Facility

K. W. Shepard

Proceedings of the Workshop on the Production and Use of Radioactive Beams at the Isospin Laboratory, Oak Ridge, TN, 7-10 October 1993, ed. J. D. Garrett. CONF-9210121, pp. 333-340 (1993)

Status and Developmental Efforts of the PIIECR Ion Source

R. Harkewicz, P. J. Billquist, and R. C. Pardo

Proceedings of the 11th International Workshop on Electron Cyclotron Resonance Ion Sources (ECRIS 11), Groningen, The Netherlands, 6-7 May 1993, ed. A. G. Drentje, KVI-Report 996, pp. 53-54 (1993)

First Operational Experience with the Positive-Ion Injector for ATLAS

L. M. Bollinger, R. C. Pardo, K. W. Shepard, J. M. Bogaty, B. E. Clifft, F. H. Munson,

G. Zinkann, and J. A. Nolen

Proceedings of the 1992 International Nuclear Physics Conference (INPC),

Wiesbaden, Germany, July 26-Aug. 1, 1993; Nucl. Phys. A553, 859C (1993)

The Positive-Ion Injector of ATLAS. Design and Operating Experience

L. M. Bollinger, R. C. Pardo, K. W. Shepard, P. J. Billquist, J. M. Bogaty, B. E.

Clifft,

R. Harkewicz, F. H. Munson, J. A. Nolen, and G. P. Zinkann

Nucl. Instrum. Methods B79, 753-757 (1993) 
Applications of Matrix Optics to Acceptance Studies in Low-Beta Ion Linacs

K. Joh and J. A. Nolen

Proceedings of the 1993 IEEE Particle Accelerator Conference, Washington, DC,

17-20 May 1993, IEEE, 93CH3279-7, Vol. 1, pp. 71-73

Beam Dynamics Studies of Four-Gap Low-Beta Superconducting Resonators

J. A. Nolen and K. Joh

Proceedings of the 1993 IEEE Particle Accelerator Conference, Washington,

DC,

17-20 May 1993, IEEE, 93CH3279-7, Vol. 1, pp. 89-91

Construction of a Superconducting RFQ Structure

K. W. Shepard, W. L. Kernedy, and K. R. Crandall

Proceedings of the 1993 IEEE Particle Accelerator Conference, Washington, DC,

17-20 May 1993, IEEE, 93CH3279-7, Vol. 2, pp. 1042-1044

Niobium Coaxial Quarter-Wave Cavities for the New Delhi Booster Linac

K. W. Shepard, A. Roy, and P. N. Potukuchi

Proceedings of the 1993 IEEE Particle Accelerator Conference, Washington,

DC,

17-20 May 1993, IEEE, 93CH3279-7, Vol. 2, pp. 1046-1047

Operational Status of the Uranium Beam Upgrade of the ATLAS Accelerator

R. C. Pardo, L. M. Bollinger, J. A. Nolen, K. W. Shepard, P. Billquist, J. M. Bogaty,

B. E. Clifft, R. Harkewicz, F. H. Munson, J. E. Specht, and G. P. Zinkann

Proceedings of the 1993 IEEE Particle Accelerator Conference, Washington, DC,

17-20 May 1993, IEEE, 93CH3279-7, Vol. 3, pp. 1694-1696

\section{MEDIUM-ENERGY PHYSICS}

Bose-Einstein Correlation of Kaons in $\mathrm{Si}+\mathrm{Au}$ Collisions at $14.6 \mathrm{~A} \mathrm{GeV} / \mathrm{c}$

Y. Áriba et al. (E-802 Collaboration)

Phys. Rev. Lett. 70, 1057-1050 (1993)

Azimuthal Asymmetries of Particles Emitted in Relativistic Heavy-Ion Collisions

T. Abbott et al. (E-802 Collaboration)

Phys. Rev. Lett. 70, 1393-1396 (1993)

Antiproton Production in p + A Collisions at $14.6 \mathrm{GeV} / \mathrm{c}$

T. Abbott et al. (E-802 Collaboration)

Phys. Rev. C 47, R1351-R1355 (1993)

Two-Body Disintegr 'ion of the Deuteron with 0.8-1.8 GeV Photons

S.J. Freedman, D.F. Geesman, R. Gilman, M.C. Greene, R.J. Holt, H.E. Jackson,

E.R. Kinney, R. Kowalczyk, C. Marchand, J. Napolitano, J. Nelson, D. Potterveld, B. Zeidman,

R. E. Segel, T.-Y. Tung. D. Beck, G. Boyd, D. Collins, B.W. Filippone, J. Jourdan,

R.D. McKeown, R. Milner, R. Walker, C. Jones, P.E. Bosted, Z.-E. Meziani, and R. Minehart

Phys. Rev. C 48, 1864-1878 (1993) 
A Polarized Gas Internal Target Using a Storage Cell in an Electron Storage Ring

R. Gilman, R. J. Holt, E. R. Kinney, R. S. Kowalczyk, J. Napolitano, A. W. Nikitin,

D. M. Nikolenko, S. G. Popov, D. H. Potterveld, I. A. Rachek, D. K. Toporkov,

E. P. Tsentalovich, B. B. Wojtsekhowski, and L. Young

Nucl. Instrum. Methods A 327, 277-286 (1993)

Two Facets of the Deuteron

R. J. Holt

Proceedings of the Workshop on Electronuclear Physics with Internal Targets and the

BLAST Detector, Arizona, USA, March 19-21, 1992, eds. Ricardo Alarcon and Malcolm

Butler (World Scientific Singapore 1993) pp. 43-57

Photon-Hadron Interactions Revisited in Deep Inelastic Muon Scattering

D. F. Geesaman

Proceedings on the Workshop on the Future of Muon Physics, Heidelberg, Germany, May 7-10, 1991, Eds. K. Jungmann, V. W. Hughes, and G. zu Putlitz (Springer-Verlag); Z. Phys. C, 56, S3-S4, pp. 159-168 (1992)

Two Body Deuteron Photodisintegration Up to $4 \mathrm{GeV}$

David H. Potterveld

Proceedings of the CEBAF 1992 Summer Workshop, Newport News, VA, June 15-19, 1992, eds. Franz Gross and Roy Holt, AIP Conference Proceedings No. 269, Particles and Fields Series 51, pp. 291-307

A Short-Orbit Spectrometer for Hall C

H. E. Jackson, R. S. Kowalczyk, D. H. Potterveld, B. Zeidman, R. L. Boudrie, and V. Hart Proceedings of the CEBAF 1992 Summer Workshop, Newport News, VA, June 15-19, 1992, eds. Franz Gross and Roy Holt, AIP Conference Proceedings No. 269, Particles and Fields Series 51 , pp. 522-526

\section{THEORETICAL PHYSICS}

Chiral Symmetry and Threshold $\pi^{0}$ Electroproduction

Veronique Bernard, Norbert Kaiser, T.-S. H. Lee, Ulf-G. Meissner

Phys. Rev. Lett. 70, 387-390 (1993)

Short Range Exchange Contributions to the Cross Section for $\mathrm{pp} \rightarrow \mathrm{pp} \pi^{0}$ Near Threshold

T.-S. H. Lee and D. O. Riska

Phys. Rev. Lett. 70, 2237-2240 (1993)

The Origins of Spin-Orbit Splitting in $15_{\mathrm{N}}$

Steven C. Pieper and V. R. Pandharipande

Phys. Rev. Lett. 70, 2541-2544 (1993)

The Coulomb Sum and Proton-Proton Correlations in Few-Body Nuclei

R. Schiavilla, R. B. Wiringa, J. Carlson

Phys. Rev. Lett. 70, 3856-3859 (1993)

Very Extended Minima in the $A=180$ Mass Region

R. R. Chasman

Phys. Lett. B 302, 134-139 (1993) 
Dissociation Reactions of the ${ }^{11} \mathrm{Be}$ One-Neutron Halo: The Interplay Between Structure and Reaction Mechanism

R. Anne, S. E. Arnell, R. Bimbot, S. Dogny, H. Emling, H. Esbensen, D. Guillemaud-Mueller,

P. G. Hansen, P. Hornshoj, F. Humbert, B. Jonson, M. Keim, M. Lewitowicz, P. Moller,

A. C. Mueller, R. Neugart, T. Nilsson, G. Nyman, F. Pougheon, K. Riisager,

M.-G. Saint-Laurent, G. Schrieder, O. Sorlin, O. Tengblad, K. Wilhelmsen Rolander,

and D. Wolski

Phys. Lett. B $\underline{304}$, 55-59 (1993)

Parity Tnuersion in the $\mathrm{N}=7$ Isotones and the Pairing Blocking Effect

H. Sagawa. B. A. Brown, H. Esbensen

Phys. Lett. B $\underline{309}, 1-4$ (1993)

Sub-barrier Fusion Cross Sections in ${ }^{58,64} \mathrm{Ni}+{ }^{92,100} \mathrm{Mo}$ Studied with the Gas-filled Magnet Technique

K. E. Rehm, H. Esbensen, J. Gehring, B. Glagola, D. Henderson, W. Kutschera, M. Paul, F. Soramel, and A. H. Wuosmaa

Phys. Lett. B 317, 31-35 (1993)

Configuration Interaction Effects in Rotational Bands of Superdeformed Nuclei

R. R. Chasman

Phys. Lett. B 319, 41-46 (1993)

Semiclassical Particle-Rotor Model of One-Neutron Transfer Reactions

A. R. Kincaid, H. Schecter, M. W. Guidry, S. Landowne, R. Donangelo, and G. Leander

Phys. Rev. C 47, 277 (1993)

Fusion Resonance in ${ }^{5} \mathrm{He},{ }^{5} \mathrm{Li}$

Dieter Kurath

Phys. Rev. C 47, 1306 (1993)

Electromagnelic Excitation of ${ }^{11} \mathrm{Li}$

D. Sackett, K. Ieki, A. Galonsky, C. A. Bertulani, H. Esbensen, J. J. Kruse, W. G. Lynch,

D. J. Morrissey, N. A. Orr, B. M. Sherill, H. Schulz, A. Sustich, J. A. Winger, F. Deak,

A. Horvath, A. Kiss, Z. Seres, J. J. Kolata, and R. E. Warner

Phys. Rev. C 48, 118-135 (1993)

Momentum Distributions for $\left({ }^{11} \mathrm{Li},{ }^{9} \mathrm{Li}+\mathrm{n}+\mathrm{n}\right)$ Breakup Reactions

H. Esbensen, G. F. Bertsch, K. Ieki

Phys. Rev. C 쏘, 326-331 (1993)

Gauge Covariance and the Fermion-Photon Vertex in Three- and Four-Dimensional, Massless

Quantum Electrodynamics

Conrad J. Burden and Craig D. Roberts

Phys. Rev. D 477, 5581-5588 (1993)

$\pi \pi$ Scattering in a QCD-Based Model Field Theory

C. D. Roberts, R. T. Cahill, M. E. Sevior, and N. Iannella

Phys. Rev. D 49, 125-137 (1994) 
From Deuterons to Neutron Stars: Variations in Nuclear Many-Body Theory

R. B. Wiringa

Rev. Mod. Phys. 65, 231-242 (1993)

Electron-Scattering Studies of Correlations in Nuclei

O. Benhar, V. R. Pandharipande, and S. C. Pieper

Rev. Mod. Phys. 65, 817-828 (1993)

Proton Spin within Nonperturbative QCD

A. E. Dorokhov, N. I. Kochelev, and Yu. A. Zubov

Int. J. Mod. Phys. A8, 603-651 (1993)

Coulomb Dissociation and Momentum Distributions for ${ }^{11} \mathrm{Li} \rightarrow{ }^{9} \mathrm{Li}+\mathrm{n}+\mathrm{n}$ Breakup Reactions

Henning Esbensen

Proceedings of the RIKEN International Workshop on Heavy-Ion Reactions with Neutron-

Rich Beams, Wako, Japan, 18-20 February 1993, eds. M. Ishihara, N. Takigawa, S. Yamaji

(World Scientific Publishing, Singapore 1993) pp. 162-173

Microscopic Calculations of Nuclear Structure and Nuclear Correlations

R. B. Wiringa

Proceedings of the CEBAF 1992 Summer Workshop, Newport News, VA, 15-19 June 1992, eds. Franz Gross and Roy Holt, AIP Conference Proceedings No. 269, Particles and Fields Series 51, (1993) pp. 210-226

Settled and Open Questions in ${ }^{8} \mathrm{Li}$

Dieter Kurath

Proceedings of the Symposium to honor Professor Stanley S. Hanna, Stanford University,

16 March 1991, eds. S. Class and L. Cohen (World Scientific, Singapore 1993)

Structure and Reactions of Nuclei Near the Neutron Drip Line

Henning Esbensen

Proceedings of the 6th International Conference on Nuclei Far From Stability, and 9th

International Conference on Atomic Masses and Fundamental Constants, Berkastel-Kues,

Germany, 19-24 July 1992, Inst. Phys. Conf. Ser. No. 132: Section 3, pp 283-292

Electromagnetic Excitation of Baryon Resonances

T.-S. H. Lee

Proceedings of Baryons '92, International Conference on the Structure of Baryons and Related

Mesons, June 1-4, 1992, Yale University (World Scientific, Singapore 1993)

pp. 99-110

Schwinger Dyson Equations: Dynamical Chiral Symmetry Breaking and Confinement

Craig D. Roberts

Proceedings of the Workshop on CCD Vacuum Structure, 1-5 June 1992, The American

University of Paris, France, eds. H. M. Fried and B. Müller (World Scientific, Singapore 1993) pp. 114-133

\section{ATOMIC AND MOLECULAR PHYSICS}

Comparisons of the QED and Relativistic Parts of the Triplet-State Energies in the Heliumlike Sequence

H. G. Berry, R. W. Dunford, and A. E. Livingston

Phys. Rev. A 47, 698 (1993) 
Branching Ratio for the M1 Decay of the $2{ }^{2} S_{1 / 2}$ State in One-Electron Krypton

S. Cheng, H. G. Berry, R. W. Dunford, D. S. Gemmell, E. P. Kanter, B. J. Zabransky,

A. E. Livingston, L. J. Curtis, J. Bailey, and J. A. Nolen, Jr.

Phys. Rev. A 47, 903 (1993)

Experimental Transition Probabilities of Intercombination Transitions in Mg-Like and Al-Like lons of Bromine

E. Träbert, J. Suleiman, S. Cheng, H. G. Berry, R. W. Dunford, E. P. Kanter, C. Kurtz,

A. E. Livingston, K. W. Kukla, F. G. Serpa, and L. J. Curtis

Phys. Rev. A 47, 3805 (1993)

Hyperfine-Structure Studies of ZrII: Experimental and Relativistic Configuration-Interaction Results

L. Young, C. A. Kurtz, D. R. Beck, and D. Datta

Phys. Rev. A 48, 173 (1993)

Double Electron Capture in $\mathrm{Ne}^{8+}$-He Collisions at Intermediate Energies

M. L. A. Raphaelian, H. G. Berry, N. Berrah, and D. Schneider

Phys. Rev. A 48, 1292 (1993)

Two-Photon Decay of the $2{ }^{1} S_{0}$ State in He-Like Bromine

R. W. Dunford, H. G. Berry, S. Cheng, E. P. Kanter, C. Kurtz, B. J. Zabransky,

A. E. Livingston, and L. J. Curtis

Phys. Rev. A 48, 1929 (1993)

Forbidden Transitions in One- and Two-Electron Nickel

R. W. Dunford, H. G. Berry, D. A. Church, M. Hass, C. J. Liu, M. L. A. Raphaelian,

B. J. Zabransky, L. J. Curtis, and A. E. Livingston

Phys. Rev. A 뚀, 2729 (1993)

$\mathrm{CH}^{+}$is Bent!

T. Graber, E. P. Kanter, Z. Vager, and D. Zajfman

J. Chem. Phys. 98, 7725-7729 (1993)

Direct Observation of Nuclear Rearrangement in Molecules

Z. Vager, T. Graber, E. P. Kanter, and D. Zajfman

Phys. Rev. Lett. 70, 3549-3552 (1993)

Experimental Evidence for Anomalous Nuclear Delocalizatic $\mathrm{n}$ in $\mathbf{C}_{2} \mathrm{H}_{3}^{+}$

Z. Vager, D. Zajfman, T. Graber, and E. P. Kanter

Phys. Rev. Lett. 71, 4319 (1993)

High-Energy Behavior of the Double Photoionization of Helium from 2 to $12 \mathrm{keV}$

J. C. Levin, I. A. Sellin, B. M. Johnson, D. W. Lindle, R. D. Miller, N. Berrah, Y. Azuma,

H. G. Berry, and D.-H. Lee

Phys. Rev. A 477, 698 (1993)

Lattice Position of Si in GaAs Determined by X-Ray Standing Wave Measurements

A. Shih, P. L. Cowan, S. Southworth, L. Fotiadis, C. Hor, B. Karlin, F. Moore, E. Dubisz, and $\mathrm{H}$. Dietrich

J. Appl. Phys. 73, 8161 (1993) 
187

Comparison of Double and Single Ionization in He by Photons and by Charged Projectiles I. A. Sellin, J. C. Levin, R. D. Miller, N. Keller, Y. Azuma, H. G. Berry,

N. Berrah-Mansour, and D. W. Lindle

SPring-8 Workshop on Atomic Physics at High Brilliance Synchrotron Radiation

Facilities,Japan Castle Research Center, Himeji, Japan, 23-24 March 1992 (Proceedings published 1993)

Note on Isotope Sputtering

P. Sigmund

Nucl. Instrum. Methods B82, 192 (1993)

Alloy Sputtering at High Fluence: Preferential Sputtering and Competing Effects

P. Sigmund and A. Olive

Nucl. Instrum. Methods B82, 269 (1993)

On the Mechanism of the Smooth Increase of the Mean Ion Charge in Ar Photoionization near the K Edge

M. Ya. Amusia

Phys. Lett. A183, 201 (1993) 